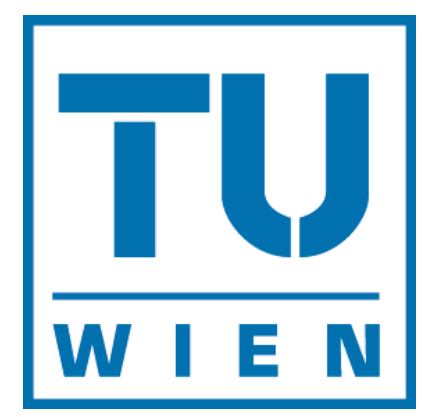

\title{
DISSERTATION
}

\section{Secondary Electron Yield on Cryogenic Surfaces as a Function of Physisorbed Gases}

ausgeführt zum Zwecke der Erlangung des akademischen Grades eines Doktors der technischen Naturwissenschaften

unter der Leitung von

Ao. Univ. Prof. Dr. phil. Herbert Störi

E134 Institut für Allgemeine Physik

Under the supervision of

Dr. Mauro Taborelli

CERN - TE - VSC

eingereicht an der Technischen Universität Wien

Fakultät für Physik von

Dipl. Ing. Asena Kuzucan

Matrikel Nr. 0226607

Rue du Belvédère 1, CH- 1203 Genf 


\section{ABSTRACT}

In LHC the electron cloud induced by photoelectrons, gas ionization and secondary electrons emitted from the beam pipe walls could be a limitation of the performance. The electron cloud induce heat load on the cryogenic system, cause pressure rise, emittance growth and beam instabilities, which in the end will limit the beam's lifetime. Beam- induced multipacting, which can arise through oscillatory motion of photoelectrons and low-energy secondary electrons bouncing back and forth between opposite walls of the vacuum chamber during successive passage of proton bunches, represent therefore a potential problem for the machine.

The secondary electron yield (SEY) is one of the key parameters for the electron cloud build up and multipacting phenomenon. An electron cloud occurs if the metal surface secondary electron yield is high enough for electron multiplication. This parameter has been extensively studied on room temperature samples but uncertainties remain for samples at cryogenic temperature. Indeed, at low surface temperature SEY is strongly dependent on the nature of the physisorbed gases and on the surface coverage.

In this work the secondary electron yield (SEY) at cryogenic temperatures has been measured and the results are presented. Of particular interest is the variation of the SEY with the gas coverage as most gases especially $\mathrm{CO}, \mathrm{CO}_{2}$, and $\mathrm{CH}_{4}$ in case of $\mathrm{LHC}$ condense on the cryogenic parts of the machine. In addition to these gases measurements have been performed with $\mathrm{N}_{2}$, because of its importance as calibration gas for most of the pressure gauges and pumps. Also measurements with $\mathrm{Kr}$ have been done, to compare the results with existing works. In order to acquire a better understanding about the behaviour of SEY as a function of different gas coverage, measurements of work function have been made. The measurements of the SEY at cryogenic surfaces require a UHV system with a sample holder which can be cooled down to cryogenic temperatures, a source of primary electrons and detection of sample current and secondary electron current. In addition a gas injection system is necessary to produce variable coverage with different gases. In the present system the sample ( $\mathrm{Cu}, \mathrm{Al}$, electro-polished $\mathrm{Cu}, \mathrm{Nb}$ and $\mathrm{C}$ ) was cooled down to 4.7K, irradiated with an electron gun and currents were measured on the sample and on a collector electrode. Work function was measured with a Kelvin Probe. 


\section{KURZFASSUNG}

Im LHC kann die Elektronenwolke, induziert durch die Photoelektronen, Gasionisation und die Sekundärelektronen, welche von den Wänden der Beamline emittiert werden, eine Begrenzung der Leistung sein. Die Elektronenwolke induziert eine Wärmebelastung des Kryo-Systems, löst Druckerhöhung, Zunahme der Emittanz und Strahlinstabilitäten aus. Schlussendlich wird die Elektronenwolke die sogenannten Strahl-Lebensdauer beschränken. Strahl induzierte Elektronenmultiplikation, welche durch die oszillierende Bewegung der Photeelektronen und energiearmer Sekundärelektronen zwischen den Wänden der Vakuumkammer ausgelöst wird, repräsentiert ein potentielles Problem für die Maschine.

Die Sekundärelektronen- Ausbeute (SEY- Secondary Electron Yield) ist einer der wichtigsten Parameter für den Aufbau der Elektronenwolke und die Multiplikation Phänomen. Die Elektronenwolke kommt zu Stande, wenn SEY der Metalloberfläche hoch genug für die Elektronenmultiplikation ist. Dieser Parameter ist mit Proben im Zimmertemperatur reichlich untersucht worden aber Frage hinsichtlich des Verhaltens bei tiefen Temperaturen bleibt ungelöst. In der Tat hängt SEY bei tiefen Temperaturen stark an der Natur der pysisorbierten Gasen und an der Bedeckung der Oberfläche ab.

In dieser Arbeit ist die SEY bei tiefen Temperaturen gemessen und die Resultate werden präsentiert. Von besonderem Interesse ist die Veränderung der SEY als eine Funktion der Gasadsorption, da die meisten Gase, vor allem $\mathrm{CO}, \mathrm{CO}_{2}$ und $\mathrm{CH}_{4}$ im $\mathrm{LHC}$ an den kryogenischen Teilen der Maschine kondensieren. Neben diesen Gasen wurden -wegen seiner Bedeutung als Kalibriergas für die meisten Manometer und Pumpen auch Messungen mit $\mathrm{N}_{2}$ durchgeführt. Noch dazu wurden Messungen mit Kr gemacht, um die Ergebnisse mit den bestehenden Publikationen vergleichen zu können. Um ein besseres Verständnis über das Verhalten der SEY als Funktion der Gasadsoption zu erwerben, wurde Messungen über die Austrittsarbeit durchgeführt. Das UHV System für die Messungen der SEY an kryogenischen Oberflächen ist mit einem Probenhalter, womit man eine Probe auf kryogenischen Temperaturen abkühlen kann, einer Elektronenquelle für die Primärelektronen und einem Messsystem für Probenstrom und Sekundärelektronenstrom ausgestattet. Zusätzlich braucht man ein Gasinjektionsystem um definierte Mengen von Gasbedeckungen mit verschiedenen Gasen produzieren zu können. In diesem System wurde die Probe ( $\mathrm{Cu}, \mathrm{Al}$, elektropolierten $\mathrm{Cu}, \mathrm{Nb}$ und $\mathrm{C}$ ) auf $4.7 \mathrm{~K}$ abgekühlt, mit einer Elektronenkanone bestrahlt und der Strom an der Probe und an dem Kollektor wurde gemessen. Die Austrittsarbeit wurde mit einer Kelvin-Sonde erfasst. 


\section{Contents}

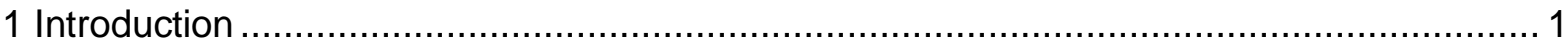

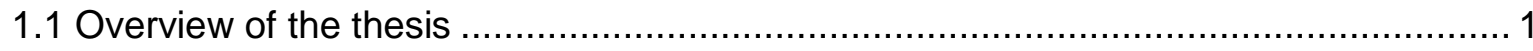

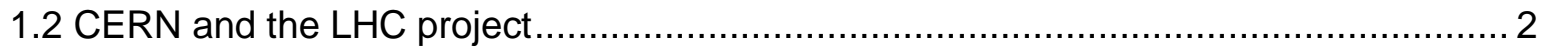

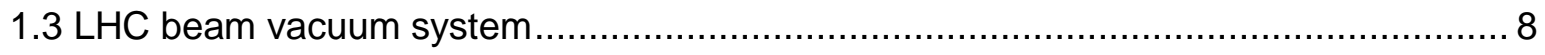

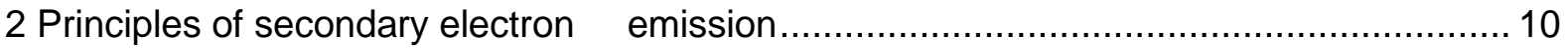

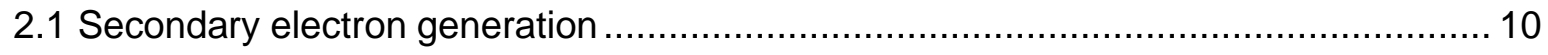

2.1.1 Incidence angle dependence of secondary electron emission ........................... 14

2.1.2 Influence of the surface roughness on the SEY .............................................. 15

2.1.3 The effect of adsorbed layers and air exposure on the SEY ............................ 17

2.1.4 Conditioning (beam scrubbing) .............................................................. 18

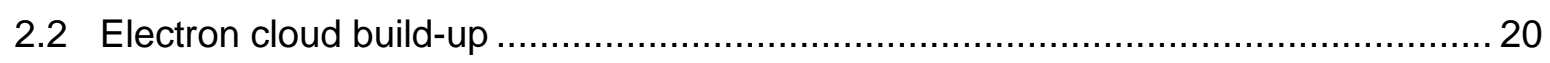

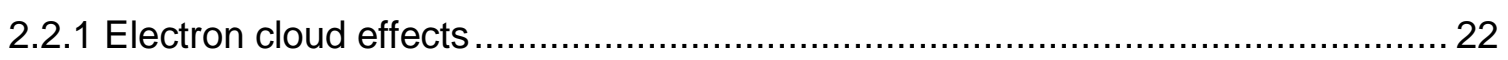

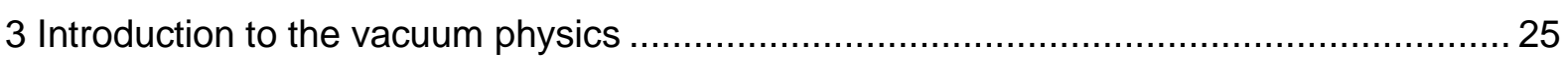

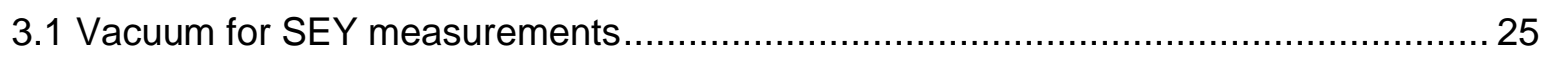

3. 2 Kinetic theory of gases and the mean free path .......................................... 26

3. 3 Conductance in vacuum systems ............................................................ 28

3.4 Calculation of pump-down time for a vacuum system ..................................... 31

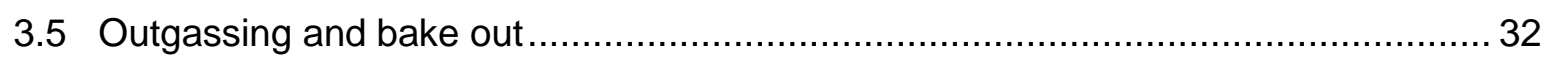

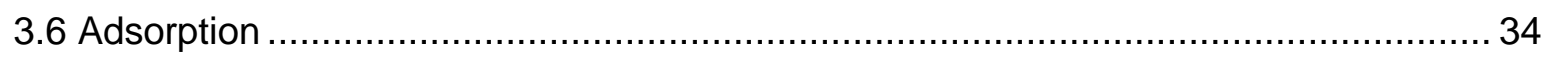

4 Design of the system for SEY- Measurements at cryogenic temperatures ................. 38

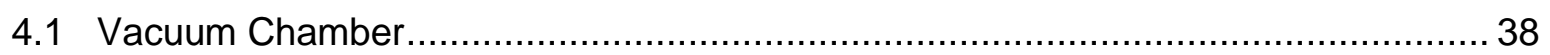

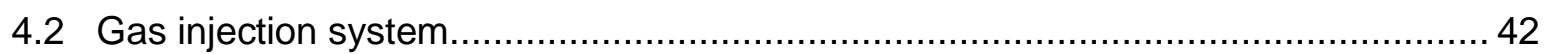

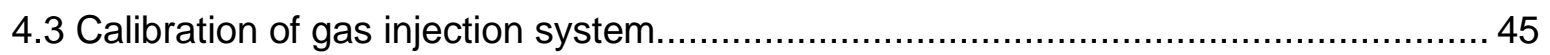

4.3.1 Measuring the gas flow into the well defined volume ....................................... 46

4.3.2 Pumping speed of the pump and the cold sample .......................................... 47

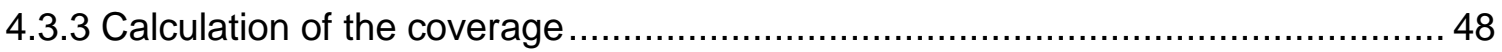

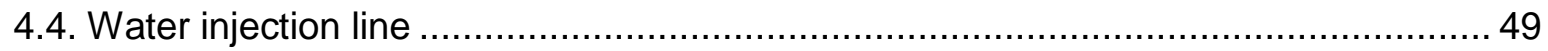

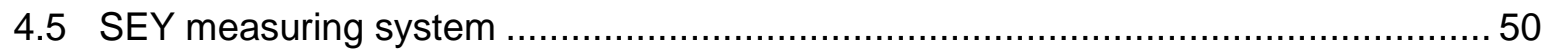

4.6 Commissioning and adjustments of the electron gun parameters ............................ 54

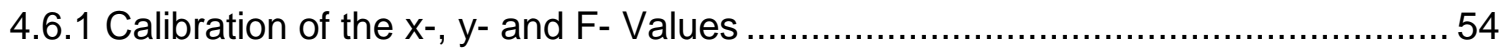

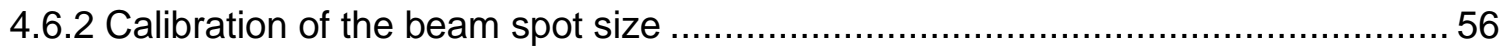

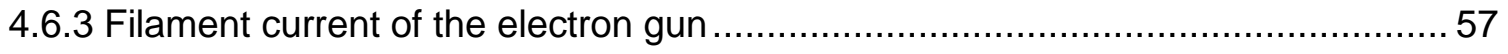

4.6.4 Calculation of the magnetic field effect on the primary electrons ....................... 59

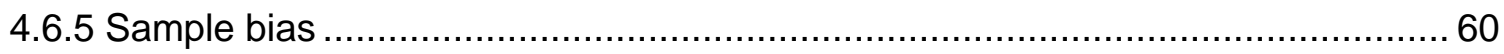

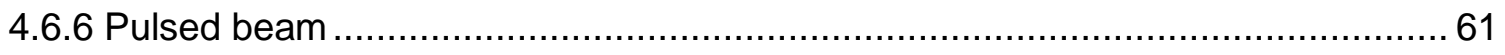




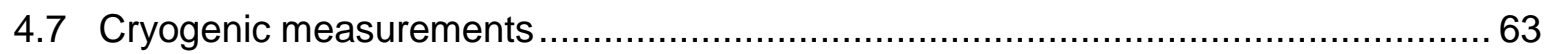

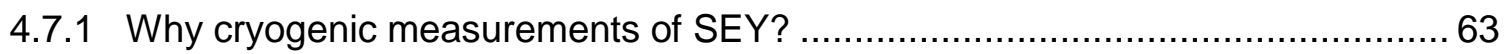

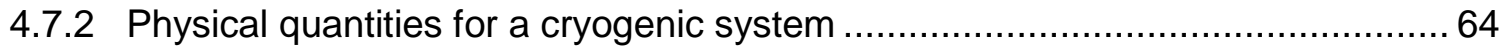

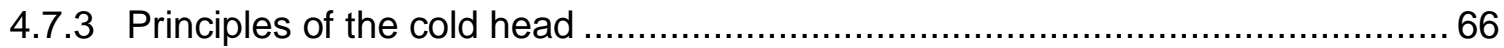

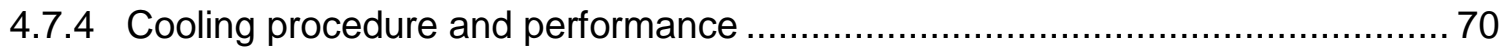

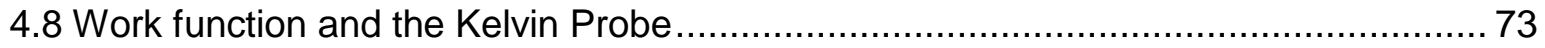

5 Secondary electron emission from cold surfaces as a function of the gas adsorption..... 75

5.1 Reference measurements with an existing system at room temperature .................. 75

5.1.1 SEY measurements with different samples ................................................. 75

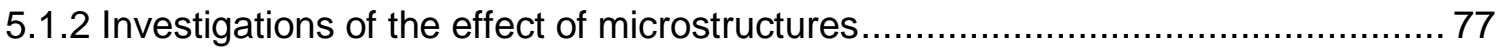

5.2 Description of the SEY experiment at cryogenic temperatures ............................... 78

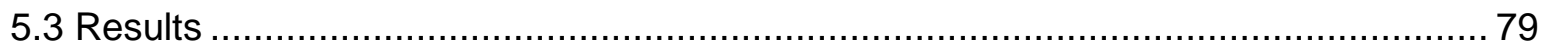

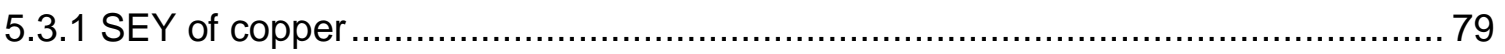

5.3.2 SEY of copper as a function of $\mathrm{Kr}, \mathrm{N}_{2}, \mathrm{CO}_{2}, \mathrm{CO}, \mathrm{CH}_{4}$ and $\mathrm{C}_{2} \mathrm{H}_{6}$ adsorption......... 80

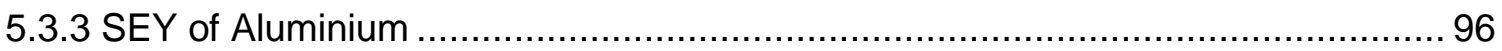

5.3.4 SEY of aluminium as a function of, $\mathrm{N}_{2}, \mathrm{CO}_{2}, \mathrm{CO}$ and $\mathrm{CH}_{4}$ adsorption.................. 96

5.3.5 SEY of electro polished copper ............................................................... 108

5.3.6 SEY SEY of electro polished copper as a function of, $\mathrm{N}_{2}, \mathrm{CO}_{2}, \mathrm{CO}$ and $\mathrm{CH}_{4}$

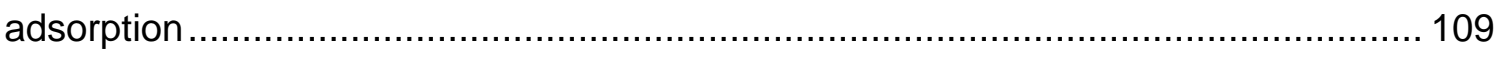

5.3.7 Comparison of the SEY of different substrates ............................................. 121

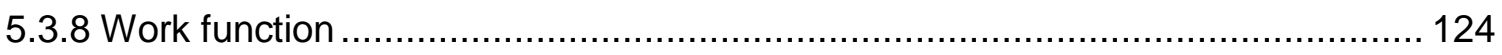

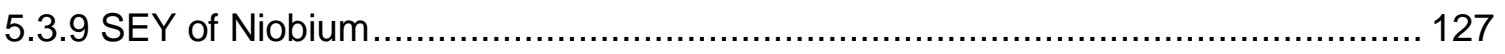

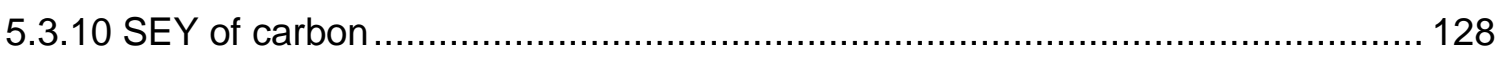

5.3.11 Adsorption and conditioning of physisorbed layers ................................... 129

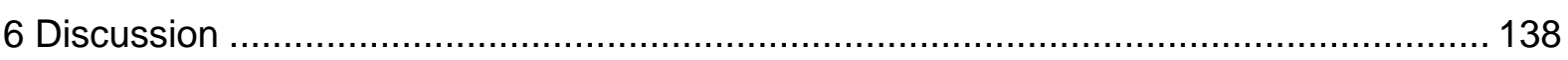

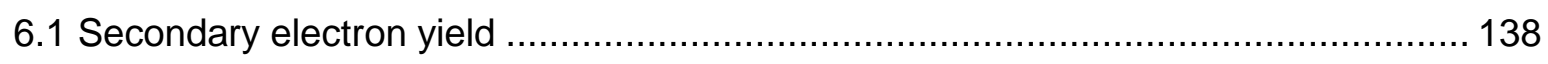

6.1.1 Tentative interpretation of the coverage dependence of SEY .......................... 138

6.1.2 Possible explanations of SEY of "bulk" adsorbate ........................................ 140

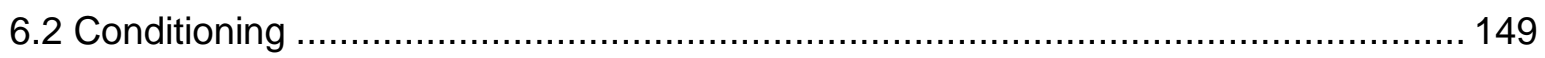

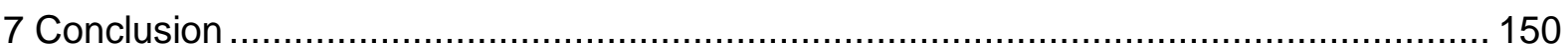

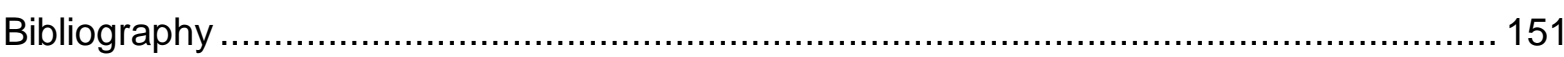

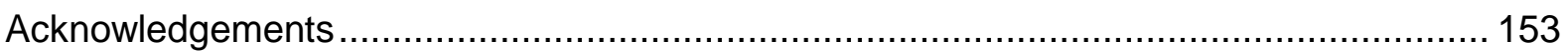

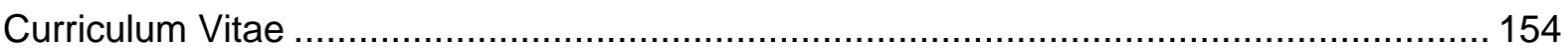




\section{Figures}

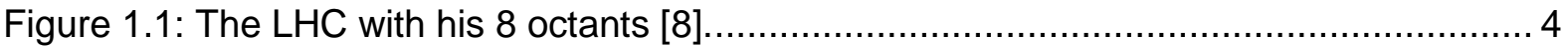

Figure 1.2. CERN accelerator complex [4] ............................................................... 7

Figure 1.3: Picture of a prototype beam screen......................................................... 9

Figure 2.1: Schematic energy distribution of electrons which are emitted from a surface bombarded with PE[20]......................................................................................... 11

Figure 2.2: The SEY of an air exposed, polycrystalline copper as a function of the primary

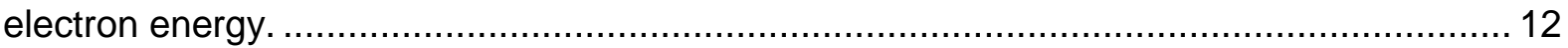

Figure 2.3: Inelastic mean free path of electrons in copper as a function of the kinetic energy [22].

Figure 2.4: Dependence of $\sigma=\delta+\eta(\delta=$ number of true secondaries/number of primaries, $\eta=$ number of backscattering/number of primaries) on the angle of incident for a $\mathrm{Si}(111)$ face [26].

Figure 2.5: Model of ripple structure for surface roughness [27] ................................... 16

Figure 2.6: Variations of SEY $\delta$ and backscattering coefficient $\eta$ of aluminium for normal incidence $\left(\theta=0^{\circ}\right)$ as a function of $H / W$ for surface roughness, for different width $W$. The dotted lines are the calculated $\delta$ and $\eta$ for a flat surface [27]...................................... 16 Figure 2.7: Influence of the surface roughness on the SEY. The probability for the electrons to escape is higher from a smooth surface[28].

Figure 2.8: SEY of glow discharged copper, after 5 minutes and 6 days of air exposure inside the vacuum vessel as a function of primary electron energy[28]............................ 18

Figure 2.9: The variation of the secondary electron yield under electron/ion bombardment[32].

Figure 2.10: SEY as a function of the primary electron energy for a copper sample colaminated on stainless steel before and after beam scrubbing. Electrons with $500 \mathrm{eV}$

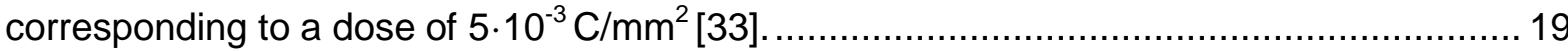

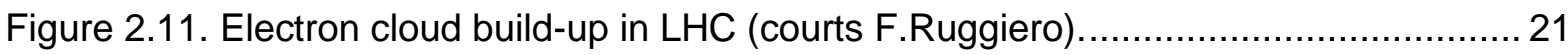

Figure 2.12: Simulation of the single bunch instability [40]. .......................................... 23

Figure 3.1: Schematic view of the molecular flow in a pipe.............................................. 30

Figure 3.2: Vacuum system for pump- down equation development. ................................ 32

Figure 3.3: Different gas production mechanism in the solid. ............................................ 32

Figure 3.4: Schematic description of adsorption and absorption. ...................................... 34

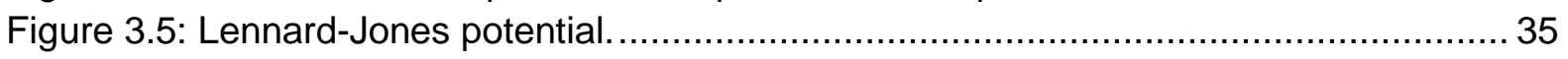

Figure 4.1: Schematic view of the measurement system for cryogenic measurements. ...... 39

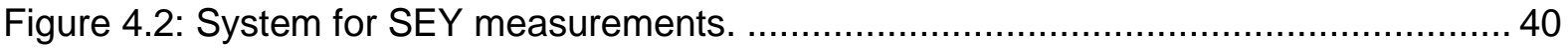

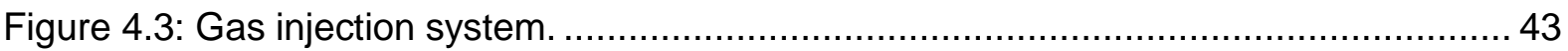

Figure 4.4: Drawing of gas injection system, which has been used for gas adsorption in

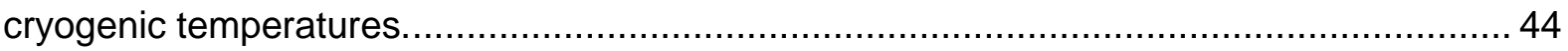

Figure 4.5: $1 / 2$ litre volume, to let a well-known amount of gas flow in the main chamber.... 45

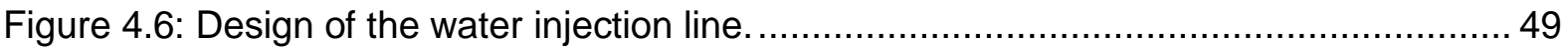

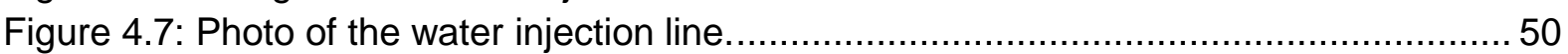

Figure 4.8: The path of electrons starting from the electron gun till the sample................. 51

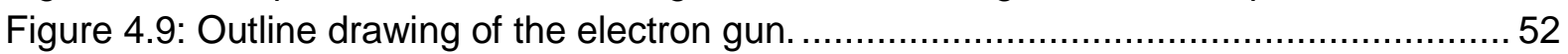

Figure 4.10: Labview programme for SEY measurements. ............................................ 53

Figure 4.11: The mask with a hole of $2 \mathrm{~mm}$ diameter, replaced in front of the sample without electrical contact to the sample. 
Figure 4.12: The calibration values for $x-, y-$, and $F$.....

Figure 4.13: The schema of the deflection plate and the path of the electron gun till the sample.

Figure 4.14: Graphs of the current on the sample depending on the voltage on the $x$ - or $y$ plate.

Figure 4.15: Secondary electron yield as a function of filament current on the electron gun.58 Figure 4.16: Total beam current as a function of filament current on the electron gun.........58

Figure 4.17: Sample current as a function of filament current on the electron gun. .............59

Figure 4.18: Displacement of the electrons with the earth magnetic field as a function of their

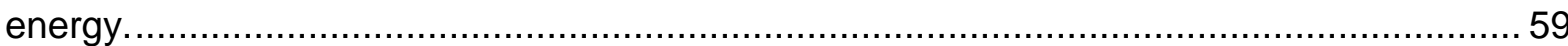

Figure 4.19: Secondary electron yield as a function of the negative bias voltage. ..............60 Figure 4.20: SEY for pulsed and continues beam and investigations of different pulse times.

Figure 4.21: The desorption yield of $\mathrm{H}_{2}, \mathrm{CH}_{4}, \mathrm{CO}, \mathrm{N}_{2}$ and $\mathrm{CO}_{2}$ as a function of the electron energy [47].

Figure 4.22: Cold head with wirings of the silicon-diode thermometer and the heater. Third photo after thermolizing the diode wires.

Figure 4.23: Cold head with dismounted thermal shield.

Figure 4.24: Piping \& Instrumentation Diagram of a cryostat for a cold head at variable temperature.

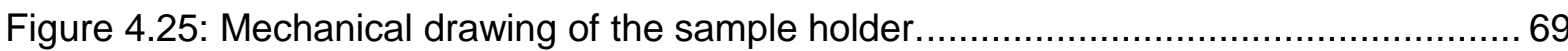

Figure 4.26: The connections of the cold head with the gasometer and the helium Dewar.. 70 Figure 4.27: The change of temperature by cooling down and warming up as a function of

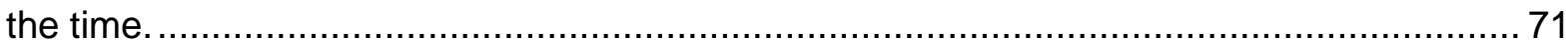

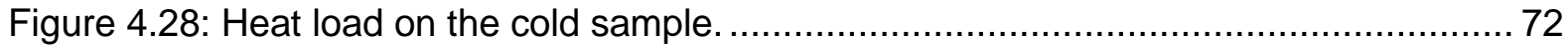

Figure 4.29: Spectrum of the residual gas of the baked vacuum chamber at room temperature and at $8 \mathrm{~K}$.

Figure 4.30: Photos of the Kelvin Probe taken before assembling on the vacuum system. . 74

Figure 4.31: Drawing of the Kelvin Probe. It is mounted on a CF63 flange. It has a work function resolution of $1-3 \mathrm{meV}$. The vibrating tip is made of stainless steel. ....................... 74

Figure 5.1: The rotating sample holder with mounted samples....................................... 76

Figure 5.2: SEY results for different samples at room temperature in a non-baked system. 76

Figure 5.3: Drilled copper sample with 92 holes $/ \mathrm{cm}^{2}$ each with $1 \mathrm{~mm}$ diameter. .................. 77

Figure 5.4: Yield of the drilled copper compared with the non drilled air exposed copper....77

Figure 5.5: SEY measurements system connected to the helium Dewar for cooling down. . 78

Figure 5.6: SEY of copper at different temperatures. The yield is decreasing with decreasing temperature.

Figure 5.7: The secondary electron yield of 10 monolayers of $\mathrm{Kr}, \mathrm{N}_{2}, \mathrm{CO}_{2}, \mathrm{CO}, \mathrm{CH}_{4}$ and $\mathrm{C}_{2} \mathrm{H}_{6}$

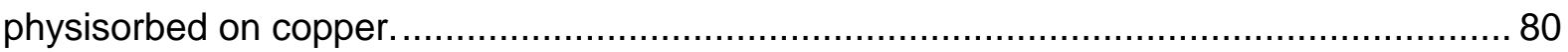

Figure 5.8: SEY of adsorbed $\mathrm{N}_{2}$ as a function of energy for different coverages. ............... 81

Figure 5.9: SEY of adsorbed $\mathrm{N}_{2}$ as a function of energy for low coverage.......................... 81

Figure 5.10: $\mathrm{SEY}$ of adsorbed $\mathrm{CO}_{2}$ as a function of energy for different coverages............. 82

Figure 5.11: SEY of adsorbed CO2 as a function of energy for low coverage. .................... 82

Figure 5.12: SEY of adsorbed CO as a function of energy for different coverages. ............. 83

Figure 5.13: SEY of adsorbed $\mathrm{CO}$ as a function of energy for low coverage. ..................... 83

Figure 5.14: SEY of adsorbed $\mathrm{CH}_{4}$ as a function of energy for different coverages. ............ 84

Figure 5.15: $\mathrm{SEY}$ of adsorbed $\mathrm{CH}_{4}$ as a function of energy for low coverage. ..................... 84

Figure 5.16: SEY of adsorbed $\mathrm{C}_{2} \mathrm{H}_{6}$ as a function of energy for different coverages............ 85 
Figure 5.17: $\mathrm{SEY}$ of adsorbed $\mathrm{C}_{2} \mathrm{H}_{6}$ as a function of energy for low coverage................... 85

Figure 5.18: SEY of adsorbed $\mathrm{Kr}$ as a function of energy for different coverages................ 86

Figure 5.19: Maximum yield as a function of coverage for different adsorbates................... 86

Figure 5.20: SEY of $\mathrm{N}_{2}$ as a function of coverage for defined primary electron energies. .... 87

Figure 5.21: SEY of $\mathrm{CO}_{2}$ as a function of coverage for defined primary electron energies... 87

Figure 5.22: SEY of $\mathrm{CO}$ as a function of coverage for defined primary electron energies.... 88

Figure 5.23: SEY of $\mathrm{CH}_{4}$ as a function of coverage for defined primary electron energies... 88

Figure 5.24: SEY of $\mathrm{C}_{2} \mathrm{H}_{6}$ as a function of coverage for defined primary electron energies. 89

Figure 5.25: SEY of $\mathrm{Kr}$ as a function of coverage for defined primary electron energies. .... 89

Figure 5.26: Maximum yield of $\mathrm{N}_{2}$ for each number of monolayer. ...................................90

Figure 5.27: Energy at the maximum yield of $\mathrm{N}_{2}$ as a function of coverage. ......................90

Figure 5.28: Maximum yield of $\mathrm{CO}_{2}$ for each number of monolayer................................. 91

Figure 5.29: Energy at the maximum yield of $\mathrm{CO}_{2}$ as a function of coverage. ....................91

Figure 5.30: Maximum yield of $\mathrm{CO}$ for each number of monolayer.................................. 92

Figure 5.31: Energy at the maximum yield of $\mathrm{CO}$ as a function of coverage........................ 92

Figure 5.32: Maximum yield of $\mathrm{CH}_{4}$ for each number of monolayer.................................. 93

Figure 5.33: Energy at the maximum yield of $\mathrm{CH}_{4}$ as a function of coverage...................... 93

Figure 5.34: Maximum yield of $\mathrm{C}_{2} \mathrm{H}_{6}$ for each number of monolayer................................ 94

Figure 5.35: Energy at the maximum yield of $\mathrm{C}_{2} \mathrm{H}_{6}$ as a function of coverage. ................... 94

Figure 5.36: Maximum yield of $\mathrm{Kr}$ for each number of monolayer..................................... 95

Figure 5.37: Energy at the maximum yield of $\mathrm{Kr}$ as a function of coverage. ....................... 95

Figure 5.38: Secondary electron yield of aluminium at room temperature and at cryogenic

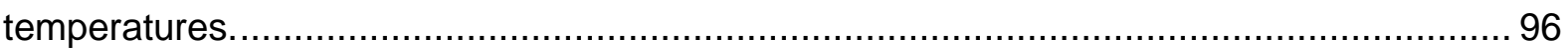

Figure 5.39: The secondary electron yield of 10 monolayers of $\mathrm{N}_{2}, \mathrm{CO}_{2}, \mathrm{CO}$ and $\mathrm{CH}_{4}$

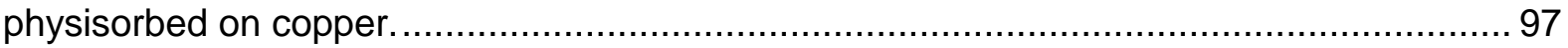

Figure 5.40: SEY of adsorbed $\mathrm{N}_{2}$ as a function of energy for different coverages............... 98

Figure 5.41: SEY of adsorbed $\mathrm{N}_{2}$ as a function of energy for low coverage........................ 98

Figure 5.42: SEY of adsorbed $\mathrm{CO}_{2}$ as a function of energy for different coverages............ 99

Figure 5.43: SEY of adsorbed $\mathrm{CO}_{2}$ as a function of energy for low coverage..................... 99

Figure 5.44: SEY of adsorbed CO as a function of energy for different coverages........... 100

Figure 5.45: SEY of adsorbed $\mathrm{CO}$ as a function of energy for low coverage. ..................... 100

Figure 5.46: $\mathrm{SEY}$ of adsorbed $\mathrm{CH}_{4}$ as a function of energy for different coverages........... 101

Figure 5.47: $\mathrm{SEY}$ of adsorbed $\mathrm{CH}_{4}$ as a function of energy for low coverage................... 101

Figure 5.48: SEY of $\mathrm{N}_{2}$ as a function of coverage for defined primary electron energies... 102

Figure 5.49: SEY of $\mathrm{CO}$ as a function of coverage for defined primary electron energies.. 102

Figure 5.50: SEY of $\mathrm{CO}_{2}$ as a function of coverage for defined primary electron energies. 103

Figure 5.51: SEY of $\mathrm{CH}_{4}$ as a function of coverage for defined primary electron energies. 103

Figure 5.52: Maximum yield of $\mathrm{N}_{2}$ for each number of monolayer. ................................. 104

Figure 5.53: Energy at the maximum yield of $\mathrm{N}_{2}$ as a function of coverage. ..................... 104

Figure 5.54: Maximum yield of $\mathrm{CO}_{2}$ for each number of monolayer.............................. 105

Figure 5.55: Energy at the maximum yield of $\mathrm{CO}_{2}$ as a function of coverage. ................... 105

Figure 5.56: Maximum yield of $\mathrm{CO}$ for each number of monolayer.................................. 106

Figure 5.57: Energy at the maximum yield of $\mathrm{CO}$ as a function of coverage...................... 106

Figure 5.58: Maximum yield of $\mathrm{CH}_{4}$ for each number of monolayer............................... 107

Figure 5.59: Energy at the maximum yield of $\mathrm{CH}_{4}$ as a function of coverage.................... 107

Figure 5.60: Maximum yield as a function of coverage for different adsorbates................. 108 
Figure 5.61: Secondary electron yield of electro polished copper at room temperature and at cryogenic temperatures. The yield is lower than the yield of copper due to the cleaner surface.

Figure 5.62: The secondary electron yield of 10 monolayers of $\mathrm{N}_{2}, \mathrm{CO}_{2}, \mathrm{CO}$ and $\mathrm{CH}_{4}$

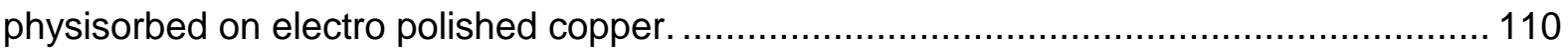

Figure 5.63: SEY of adsorbed $\mathrm{N}_{2}$ as a function of energy for different coverages............. 111

Figure 5.64: SEY of adsorbed $\mathrm{N}_{2}$ as a function of energy for low coverage..................... 111

Figure 5.65: SEY of adsorbed $\mathrm{CO}_{2}$ as a function of energy for different coverages........... 112

Figure 5.66: SEY of adsorbed $\mathrm{CO}_{2}$ as a function of energy for low coverage..................... 112

Figure 5.67: SEY of adsorbed CO as a function of energy for different coverages. ...........113

Figure 5.68: SEY of adsorbed $\mathrm{CO}$ as a function of energy for low coverage. ................... 113

Figure 5.69: SEY of adsorbed $\mathrm{CH}_{4}$ as a function of energy for different coverages.......... 114

Figure 5.70: $\mathrm{SEY}$ of adsorbed $\mathrm{CH}_{4}$ as a function of energy for low coverage. ...................114

Figure 5.71: SEY of $\mathrm{N}_{2}$ as a function of coverage for defined primary electron energies... 115

Figure 5.72: SEY of $\mathrm{CO}_{2}$ as a function of coverage for defined primary electron energies. 115

Figure 5.73: SEY of $\mathrm{CO}$ as a function of coverage for defined primary electron energies.. 116

Figure 5.74: SEY of $\mathrm{CH}_{4}$ as a function of coverage for defined primary electron energies. 116

Figure 5.75: Maximum yield of $\mathrm{N}_{2}$ for each number of monolayer. ............................... 117

Figure 5.76: Energy at the maximum yield of $\mathrm{N}_{2}$ as a function of coverage. ..................... 117

Figure 5.77: Maximum yield of $\mathrm{CO}_{2}$ for each number of monolayer................................ 118

Figure 5.78: Energy at the maximum yield of $\mathrm{CO}_{2}$ as a function of coverage. ................... 118

Figure 5.79: Maximum yield of $\mathrm{CO}$ for each number of monolayer.................................... 119

Figure 5.80: Energy at the maximum yield of $\mathrm{CO}$ as a function of coverage..................... 119

Figure 5.81: Maximum yield of $\mathrm{CH}_{4}$ for each number of monolayer.............................. 120

Figure 5.82: Energy at the maximum yield of $\mathrm{CH}_{4}$ as a function of coverage..................... 120

Figure 5.83: Maximum yield as a function of coverage for different adsorbates................ 121

Figure 5.84: Maximum yield of different $\mathrm{N}_{2}$ coverages physisorbed on different substrates.

122

Figure 5.85: Maximum yield of different $\mathrm{CO}_{2}$ coverages physisorbed on different substrates. 122

Figure 5.86: Maximum yield of different $\mathrm{CO}$ coverages physisorbed on different substrates. 123

Figure 5.87: Maximum yield of different $\mathrm{CH}_{4}$ coverages physisorbed on different substrates. 123

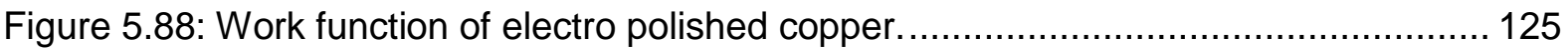

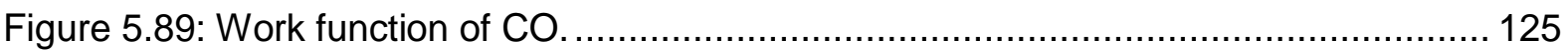

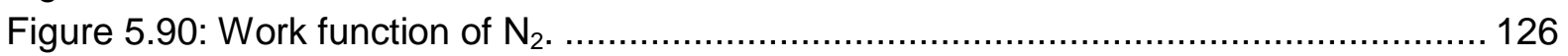

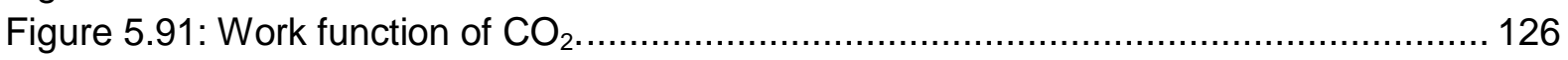

Figure 5.92: SEY of Niobium as a function of the primary electron energy at different

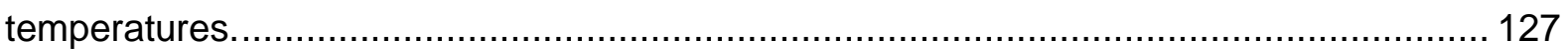

Figure 5.93: SEY of carbon at room temperature and at cryogenic temperatures. ............. 128

Figure 5.94: Residual gas spectrum of the main chamber before and during gas injection.

Figure 5.95: Maximum yield as a function of electron dose on $25 \mathrm{ML} \mathrm{H}_{2} \mathrm{O} / \mathrm{Cu}$................. 130

Figure 5.96: Secondary electron yield of water during conditioning................................ 131

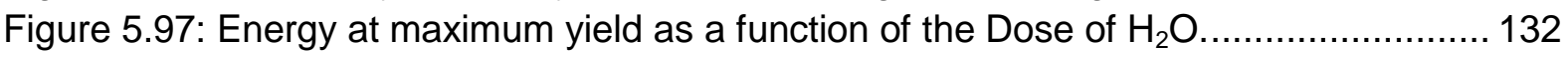

Figure 5.98: Secondary electron yield of carbon monoxide during conditioning................. 133

Figure 5.99: Maximum yield as a function of electron dose of CO .................................. 134 
Figure 5.100: Energy at the maximum yield as a function of total electron dose of CO. ... 135

Figure 5.101: Secondary electron yield of $\mathrm{N}_{2}$ during conditioning................................... 136

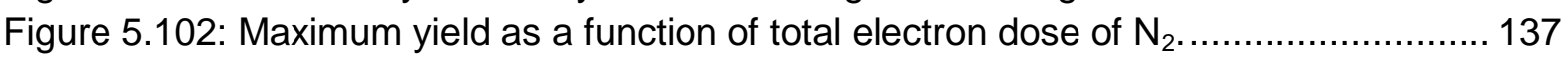

Figure 5.103: Energy at maximum yield as a function of total electron dose of $\mathrm{N}_{2} \ldots \ldots \ldots \ldots .137$

Figure 6.1: Three parts of the curve for the maximum yield as a function of the number of monolayers.

Figure 6.2: Generation of secondary electrons in insulators via electron beam coming from an electron gun. $\chi$ is the electron affinity and it is either very low or even negative in noble gases.

Figure 6.3: Maximum yield as a function of the stopping power. 142

Figure 6.4: Maximum yield as a function of the number of electrons for each gas. For all the gases but $\mathrm{Kr}$, the values are saturation values. For $\mathrm{Kr}$, SEY of 60 monolayers is shown. . 143 Figure 6.5: Maximum yield as a function of electron density. Value for $\mathrm{Kr}$ is taken for $60 \mathrm{ML}$.

Figure 6.6: Maximum yield of rare gases as a function of electron density. Values are taken for 60 monolayers [30].

Figure 6.7: Maximum yield of different adsorbates as a function of the range of primary electrons.Values of rare gases are taken for 60 monolayers. For values of $\mathrm{Ne}, \mathrm{Ar}$, $\mathrm{Xe}$ see [30]...... 145

Figure 6.8: Maximum yield as a function of the density. Value for $\mathrm{Kr}$ is taken for $60 \mathrm{ML} . \ldots 145$ Figure 6.9: Inelastic mean free path of different adsorbates as a function of the energy. The values are calculated using [22]. The value for $\mathrm{Kr}$ is for $60 \mathrm{ML}$ 147

Figure 6.10: Maximum yield as a function of the number of valence electrons. The value for $\mathrm{Kr}$ is for $60 \mathrm{ML}$ 148 


\section{Tables}

Table 1.1: Beam properties in LHC.......................................................................... 7

Table 3.1: The root-mean-square velocity $v_{r m s}$ of different gas molecules at 0 and $23^{\circ} \mathrm{C}$ [45].

Table 3.2: Pressure ranges

Table 3.3: Pressure ranges, mean free paths, and density for nitrogen at room temperature.

Table 3.4: Outgassing rates of different materials (All values depend on pre-treatment) [43].

Table 3.5: Number of the programme used for the bake out of different elements. ............ 42

Table 3.6: Maximum temperature and the time for each temperature for different

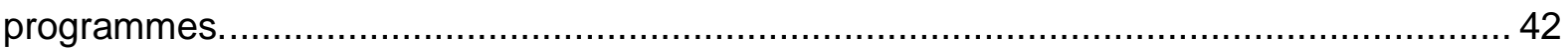

Table 3.6: Calculated and measured conductance of different gases used for the gas

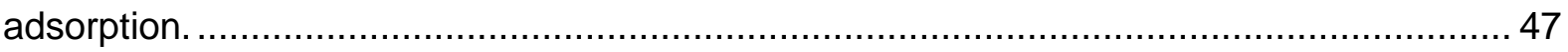

Table 3.7: Measured pumping speed of the different samples for different gases and the

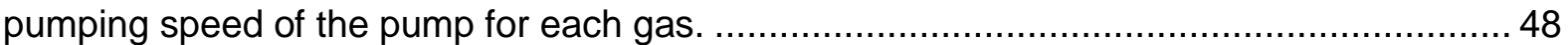

Table 5.1: Surface comparison of copper and electro polished copper. $R_{z}$ means "Maximum height of profile" and Surface index is the ratio of the surface area to the area of an ideal plane. 108

Table 6.1: Band gap of different adsorbates and their maximum SEY. The values for the maximum yield for all the gases but rare gases are the saturation values. The maximum yield of the rare gases is given for 60 monolayers. The saturation value for them is not known. 
"curiosity is always the starting point for solutions to a problem" Galileo Galilei

\section{Introduction}

\subsection{Overview of the thesis}

The scope of this thesis is the measurement and the interpretation of the secondary electron yield (SEY) on surfaces held at cryogenic temperatures. Performed in the vacuum group at the TE department of CERN, it aims a better understanding of the influence of physisorbed gases in the cold regions of the LHC vacuum system on the performance of the machine.

In chapter 1 an introduction of CERN and the LHC project is given. The importance of the vacuum system and the effect of the beam on the vacuum are described.

The basic principles of secondary electron emission and the effect of the electron cloud in particle accelerators are presented in chapter 2.

An introduction to the vacuum physics and technology is presented in chapter 3 . Also outgassing, bake out and adsorption processes are presented in chapter 3 .

The design and the operation of the experimental system, dedicated to SEY measurements, including vacuum chamber, gas injection system, calibration of the gas injection system, water injection line, electron gun, the operation mode of the cooling system and the Kelvin Probe are described in chapter 4.

In chapter 5 the experimental procedure and the results are given. The reference measurements and the cryogenic measurements with detailed operation modes and results of SEY as a function of gas adsorption are described in this chapter.

A discussion of the results is given in chapter 6 .

Finally a conclusion of the work is given in chapter 7 . 


\subsection{CERN and the LHC project}

What we are made of and where we are from? The standard model of particle physics describes the interactions between 12 elementary building blocks (quarks and leptons) and the 3 out of 4 force carriers (bosons). This theory includes [1]:

- Strong interactions due to the colour charges of quarks and gluons.

- A combined theory of weak and electromagnetic interaction, known as electroweak theory, that introduces $\mathrm{W}$ and $\mathrm{Z}$ bosons as the carrier particles of weak processes, and photons as mediators to electromagnetic interactions.

Gravity is not yet part of this framework. These effects are tiny under high-energy Physics situations, and can be neglected in describing the experiments. Eventually, scientists seek a theory that also includes a correct quantum version of gravitational interactions, but this is not yet achieved.

The Standard Model was the triumph of particle physics of the 1970's. It incorporated all that was known at that time and has since then successfully predicted the outcome of a large variety of experiments. Today, the Standard Model is a well established theory applicable over a wide range of conditions [1].

The standard model (SM) has been very successful in explaining the elementary particles and their interaction. But it is not a complete theory. We do not know what causes the fundamental particles to have masses. The basis of the Standard Model is to combine the weak force and the electromagnetic force. This unification implies that electricity, magnetism, light and some types of radioactivity are all manifestations of a single underlying force called, the electroweak force [2]. But in order for this unification to work mathematically, it requires that the force-carrying particles have no mass. We know from experiments that this is not true. Physicists Peter Higgs, Robert Brout and François Englert came up with a solution to solve this conundrum. They suggested the idea called the Higgs mechanism [2]. This mechanism involves one additional particle, called the Higgs boson, and one additional force type, mediated by exchanges of this boson. According to that mechanism all particles had no mass just after the Big Bang. As the Universe cooled and the temperature fell below a critical value, an invisible force field called the 'Higgs field' was formed together with the associated 'Higgs boson' [2, 3]. The field prevails throughout the cosmos: any particles that interact with it are given a mass via the Higgs boson. The more they interact, the heavier they become, whereas particles that never interact are left with no mass at all. The prediction of the 
existence of the scalar fundamental particles, called Higgs bosons, has not been confirmed by any experiment $[2,3]$. Today we can only say that if it exists, it must have a mass greater than about $80 \mathrm{GeV} / \mathrm{c}^{2}$.

Astronomers and physicists have found that all we see in the Universe - planets, stars, galaxies - accounts for only a tiny $4 \%$ of it! In a way, it is not so much the visible things that define the Universe, but rather the void around them.

Cosmological and astrophysical observations indicate that most of the Universe is made up of invisible substances that do not emit electromagnetic radiation - that is, we cannot detect them directly through telescopes or similar instruments. We detect them only through their gravitational effects, which makes them very difficult to study. These mysterious substances are known as 'dark matter' and 'dark energy'. Researchers now believe that the gravitational effect of dark matter makes galaxies spin faster than expected, and that its gravitational field deviates the light of objects behind it. Measurements of these effects show that dark matter exists, and they can be used to estimate the density of dark matter even though we cannot directly observe it [1-3].

But what is dark matter? One idea is that it could contain 'supersymmetric particles' hypothesized particles that are partners to those already known in the Standard Model. Supersymmetry is one of the attempts to extend the Standard Model. Experiments at the Large Hadron Collider may be able to find supersymmetric particles [2].

Some physicists are working to support a new idea called string theory. This attempts to tie up the loose ends in the Standard Model by explaining all the fundamental particles and forces (including gravity) in a unified framework. Underlying string theory is the radical idea that fundamental particles are not really like points or dots, but rather small loops of vibrating strings. All the different particles and forces are just different oscillation modes of a unique type of string. Bizarrely, the theory also implies that besides the familiar three-dimensional world and the fourth dimension of time, there are six additional spatial dimensions! These extra dimensions are apparently 'curled up' so small that we do not see them. Another attempt of scientist at CERN is to observe the extra dimensions at LHC [2].

CERN- Centre Européene pour la Recherche Nucléaire, the European Organization for Nuclear Research- with his accelerator complex, consisting of linear and circular accelerators, is one of the biggest research centres for high energy physics in the world [4]. 
CERN was founded in 1954 and employs just around 2500 people, who design and build the particle accelerators and ensure their smooth operation. Additionally about 8000 scientists, who represent 580 universities and 85 nationalities, come to CERN [4].

The Large Hadron Collider (LHC), which was placed into the existing tunnel of LEP collider, has a circumference of $26,7 \mathrm{~km}$. It has an eightfold structure and each of its octants consists of an arc, where the main bending magnets (the main dipoles), focussing and defocusing (quadrupole) magnets and higher order correction magnets are situated (Figure 1.1) [5].

The LHC is the largest particle accelerator in the world and it has been designed to provide proton-proton collision at $14 \mathrm{TeV}$ centre-of-mass energy with a luminosity of $10^{34} \mathrm{~cm}^{-2} \mathrm{~s}^{-1}$. This will be achieved by filling each of the two rings with 2835 bunches of $10^{11}$ particles each[6, 7]. In 2011 the achieved energy is $7 \mathrm{TeV}$ in total with a luminosity of $2 \cdot 10^{32} \mathrm{~cm}^{-2} \mathrm{~s}^{-1}$. This luminosity could be reached with 700 bunches of $10^{8} \mathrm{p} / \mathrm{bunch}$.

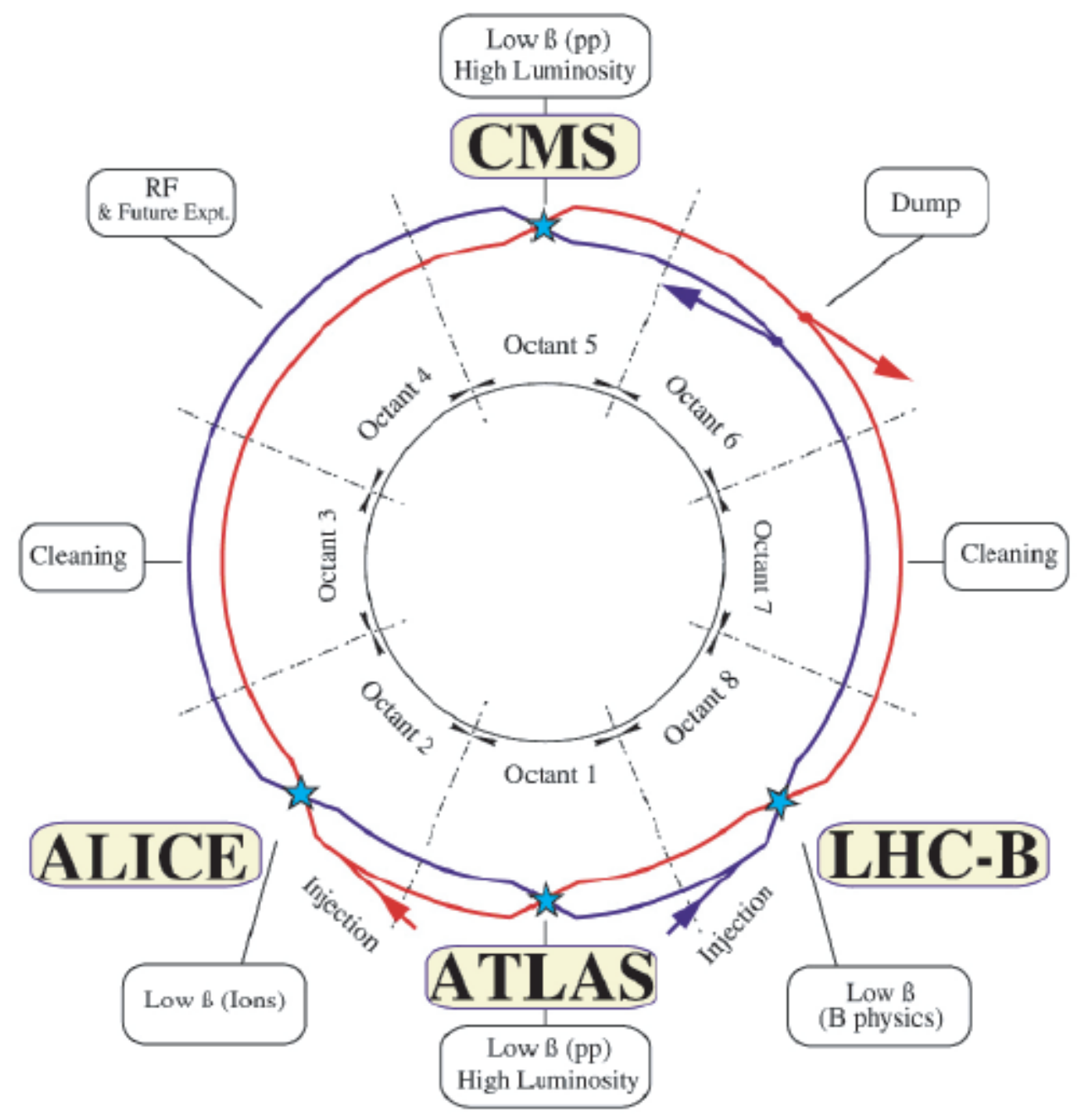

Figure 1.1: The LHC with his 8 octants [8]. 
In a synchrotron like LHC, the beam will be accelerated through radio-frequency (RF) cavities, which provide the longitudinal electric field.

To focus and steer the beams, superconducting magnets are used, which operate at a temperature of $1.9 \mathrm{~K}$. Thousands of magnets of different varieties are providing a magnetic field of nearly 8 Tesla.

Two beams of particles, which are travelling in two separate vacuum chambers, will cross and collide in four experimental areas with four detectors. The two big detectors are ATLAS and CMS. Both of them are general purpose detectors to analyse the particles produced by the collision in accelerator. ALICE is a medium sized detector to investigate the physics of heavy ion collisions. Another medium sized detector, $\mathrm{LHCb}$, is designed to detect the large number of b-hadrons produced in the LHC.

- A Toroidal LHC ApparatuS (ATLAS) [9] which can be divided into four major parts, consists of a series of concentric cylinders around the interaction point where the proton beams of LHC collide. The four parts are: The inner detector, the calorimeters, the muon spectrometer and the magnet systems. The magnet systems consist of eight very large air-core superconducting barrel loops, two end caps, which are producing the outer toroidal magnetic field, and of an inner solenoid which produces a two tesla magnetic field around the inner detector. ATLAS experiment is searching for the Higgs boson[10] and it is sensitive to largest possible Higgs masses. Another goal of ATLAS is to investigate the asymmetry between the behaviour of matter and antimatter, called as CP-violation[10], which should explain according to the standard model the lack of detectable antimatter in the universe. Also supersymmetry, dark matter and extra dimensions are investigated in ATLAS experiment. To study the top quark[10], which was discovered at Fermilab in 1995, should be also possible due to the high energy and collision rates. Furthermore a new model of physics like the broken symmetry[10] can also be investigated.

- Compact Muon Solenoid (CMS) detector[11] is designed to achieve the same goals like ATLAS. In contrary to ATLAS it consists of only one magnetic system, a superconducting solenoid, which produces a magnetic field of 4T (about 100000 times the magnetic field of the earth). The detector is made of an inner tracker with ten layers of silicon strip detectors and silicon pixel detectors close to the interaction point, an electromagnetic calorimeter with an excellent energy resolution and a muon system for momentum measurements up to highest luminosity. 
- A Large Ion Collider Experiment (ALICE)[12] is used to investigate the strong interactions of matter at extreme densities and high temperatures during heavy ion collisions. The data received from ALICE experiment will allow to study a state of matter known as quark-gluon plasma[10], which is believed to have existed soon after

the big bang. It is made of an inner tracking system consisting of six cylindrical layers of silicon detectors, radiation and momentum detectors, a muon spectrometer and an electro-magnetic calorimeter.

- LHCb experiment[13] is dedicated to get the understanding about why the universe appears to be composed almost entirely of matter but no antimatter. It is designed to study the CP-violation in B-mesons decay[10]. It is a single arm forward spectrometer made of a vertex detector, two ring imaging Cherenkov detectors and of the electromagnetic and hadronic calorimeters.

The protons with an initial energy of $100 \mathrm{keV}$, which are produced in a Duoplasmatron source, pass through a linear accelerator (LINAC 2), where they achieve energy of $50 \mathrm{MeV}$. A new linear accelerator (LINAC4) is under construction to replace LINAC2. Further they are injected into the PSB (Proton Synchrotron Booster), where their energy gets increased and leave there with energy of $1.4 \mathrm{GeV}$. Then they pass through the Proton Synchrotron (PS, built in 1959), where they reach $28 \mathrm{GeV}$, and then the Super Proton Synchrotron (SPS), where they are accelerated up to $450 \mathrm{GeV}$. SPS is a circular accelerator with $2 \mathrm{~km}$ of diameter, which started operation in 1976. Finally the proton beams will be injected in LHC, where they reach the final energy of $7 \mathrm{TeV}$. Figure 1.2 shows the CERN accelerator complex. Heavy ions, produced in LINAC3 with energy of 4,2MeV/u are transferred to the Low Energy Ion Ring (LEIR), which accelerates the ions before transferring them to the Proton Synchrotron. This accelerator was commissioned in 2005, after having been reconfigured from the previous Low Energy Anti-proton Ring (LEAR). Isotope Separator OnLine DEvice (ISOLDE) is used to investigate unstable nuclei and was first in operation in 1967 and was rebuilt and upgraded afterwards. The Antiproton Decelerator (AD) reduces the velocity of antiprotons to about $10 \%$ the speed of light for research into antimatter. 

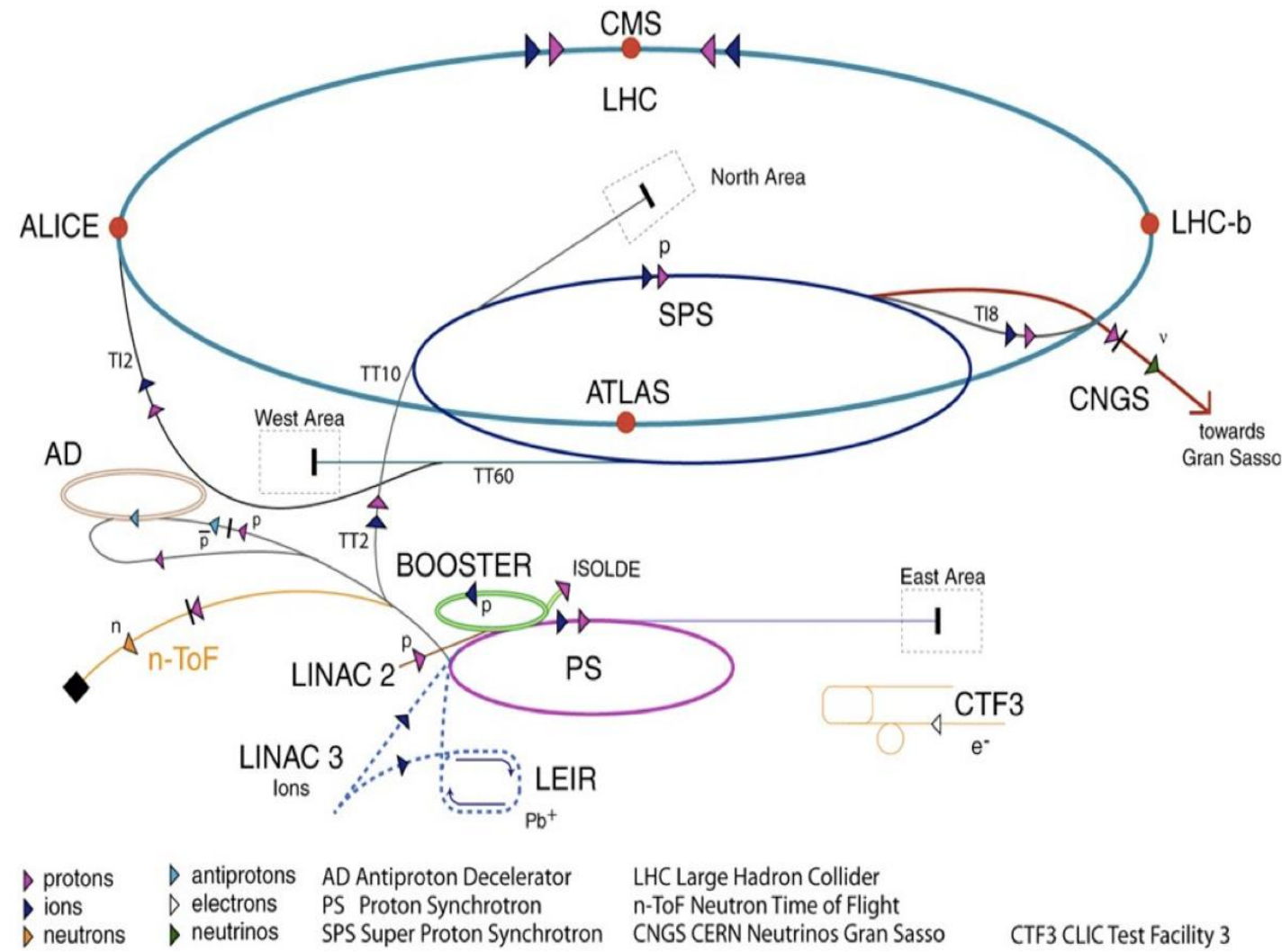

Figure 1.2. CERN accelerator complex [4].

The table below shows the nominal parameters of the LHC machine [14].

\begin{tabular}{|l|l|}
\hline Beam Energy (TeV ) & 7 \\
\hline Number of particles/bunch & $1.15 \cdot 10^{11}$ \\
\hline Number of bunches & 2835 \\
\hline Beam current I (A) & 0.54 \\
\hline Bunch spacing (ns) & 25 \\
\hline Luminosity (cm $\left.{ }^{-2} \mathrm{~s}^{-1}\right)$ & $10^{34}$ \\
\hline Bend field B (T) & 8.4 \\
\hline Circumference C (km) & 26.66 \\
\hline Bending radius (m) & 2784.32 \\
\hline No. of radiated photons per proton & 0.4 at 7TeV \\
\hline No. of radiated photons above 4eV per proton & 0.18 at 7TeV \\
\hline Relativistic factor $\mathrm{Y}$ & 7460.52 at $7 \mathrm{TeV}$ \\
\hline
\end{tabular}

Table 1.1: Beam properties in LHC. 
In the LHC it is aimed to achieve a luminosity of $10^{34} \mathrm{~cm}^{-2} \cdot \mathrm{s}^{-1}$. The luminosity is the number of events for a process per unit cross section and is a measure of the performance of the accelerator [15].

$$
L=k_{b} \frac{f_{r e v} N_{1} N_{2}}{4 \pi \sigma_{x}^{*} \sigma_{y}^{*}}
$$

$N_{1}, N 2 \ldots$ Number of particles of the rotating bunches

$k_{b}$...Number of bunches per beam

$f_{\text {rev....The revolution frequency }}$

$\sigma_{x}^{*}, \sigma_{y}^{*} \ldots$ The beam sizes at the collision point

To reach the maximum luminosity it is essential to minimize the transverse dimensions of the two beams and store as many particles as possible. There are different dynamic effects, which can reduce the luminosity of a storage ring and lead to beam instabilities. Three most important effects are the collective effects, interaction of the charged particles with themselves and with the surrounding electro-magnetic fields and the electron-cloud effect, which involve the interaction between high-energy beams and low-energy electrons produced in the vacuum chamber.

\subsection{LHC beam vacuum system}

The LHC includes three different vacuum systems: the insulation vacuum for the helium distribution line, the insulation vacuum for cryomagnets and the beam vacuum. The beam vacuum system has warm sections and cold arcs with different requirements. The chambers of the warm sections are coated with a TiZrV non evaporable getter (NEG) in order to decrease the residual gas pressure and secondary electron emission yield[16]. According to[17], the instantaneous power radiated by a charged particle, in this case a proton, travelling on a circular orbit is given by

$$
P_{\text {s.r. }}=\frac{1}{4 \pi \epsilon_{0}} \frac{2}{3} \frac{e^{2}}{c^{3}} \frac{v^{4}}{r^{2}} \gamma^{4}
$$

where $e$ is charge of the proton (i. e. the elementary charge), $c$ the speed of light in vacuum, $v$ the velocity $(v \approx c), r$ the bending radius $(r=2784,32 m$ for the main dipoles) and the 
relativistic factor ( $\gamma=7461$ for $7 \mathrm{TeV}$ protons). Substituting the numerical values into equation results in a value for the instantaneous power radiated by one proton of $P_{\text {s.r }}:=1.84 \cdot 10^{-11} \mathrm{~W}$. Having 2835 bunches with $1.05 \cdot 10^{11}$ protons per bunch distributed over the circumference of LHC $(26658,883 \mathrm{~m})$ results in a average linear proton density of about $1,12 \cdot 10^{10} \mathrm{~m}^{-1}$, hence the linear heat load caused by synchrotron radiation in the main dipoles is about $0,2 \mathrm{~W} \mathrm{~m}^{-1}$. To avoid the heat load, which would be dissipated in the superconducting magnets, if transferred to the cold mass, the cold mass is shielded against synchrotron radiation by a beam screen. The beam screen is made of a racetrack shaped tube with two cooling capillaries placed on its flat parts and it is cooled down to a temperature between $5 \mathrm{~K}$ and $20 \mathrm{~K}$ by using of pressurized helium gas[18]. At this temperature all gases except hydrogen are condensed on the beam screen. These physisorbed gases on the beam screen are modifying the surface properties and therefore change the secondary electron yield. A thin layer of oxygen free high conductivity copper is co-laminated with a low permeability stainless steel, the base material of the screen. This layer is intended to carry the beaminduced image currents, thus reducing the machine impedance to an acceptable value. Also important aspects of the beam screen are the pumping slots placed on the flat parts of the screen. Residual gas molecules can travel through this pumping slots and reach the cold bore wall where they will be permanently adsorbed (Figure 1.3).

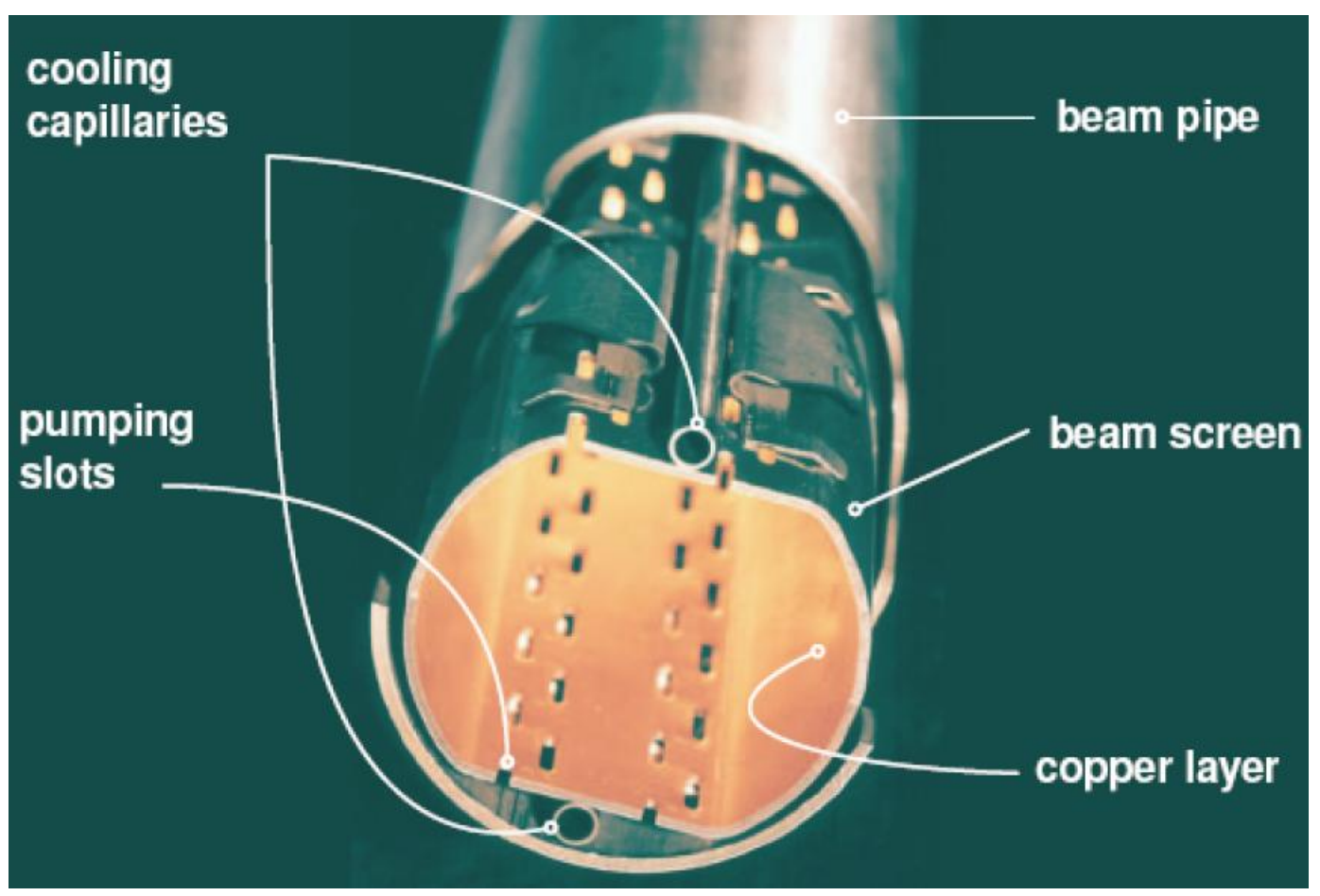

Figure 1.3: Picture of a prototype beam screen. 


\section{Principles of secondary electron emission}

\subsection{Secondary electron generation}

Secondary electron emission is the process, in which electrons leave a material under bombardment of a particle beam. Primary particles can get reflected or backscattered trough elastic scattering on the surface or they can enter the material, undergo inelastic scattering, lose energy and set lattice electrons free. These free electrons are also scattered and they interact with other electrons which cause a cascade. Not all of the free electrons can reach or leave the surface. Some of them lose their energy before reaching the surface. If they can leave the surface, they are called secondary electrons. The yield of the secondaries depends on the production of secondaries and their escape probability. The measured secondary electrons of a surface bombarded with primary electrons can be divided in following classes[19]:

1) Elastically backscattered electrons: These are electrons get scattered on the surface and come back with the same energy as the primaries

2) Rediffused electrons: Electrons, which get scattered inelastically, but lose a relatively low amount of their kinetic energy and are reflected back.

3) True secondaries: Electrons, which are produced by the inelastic interaction of primary electrons with the lattice electrons. Their typical kinetic energy is below $50 \mathrm{eV}$. 
Figure 2.1 shows the typical energy distribution of electrons, released from a surface under bombardment of primary electrons with energies $100 \mathrm{eV}<\mathrm{E}_{\mathrm{pe}}<1 \mathrm{keV}$. Electron with energies $\mathrm{E}<50 \mathrm{eV}$ are the so called true secondaries (SE) and electrons with energies $50 \mathrm{eV}<\mathrm{E}<\mathrm{E}_{\mathrm{pe}}$ are the inelastically backscattered and elastically reflected electrons (RE).

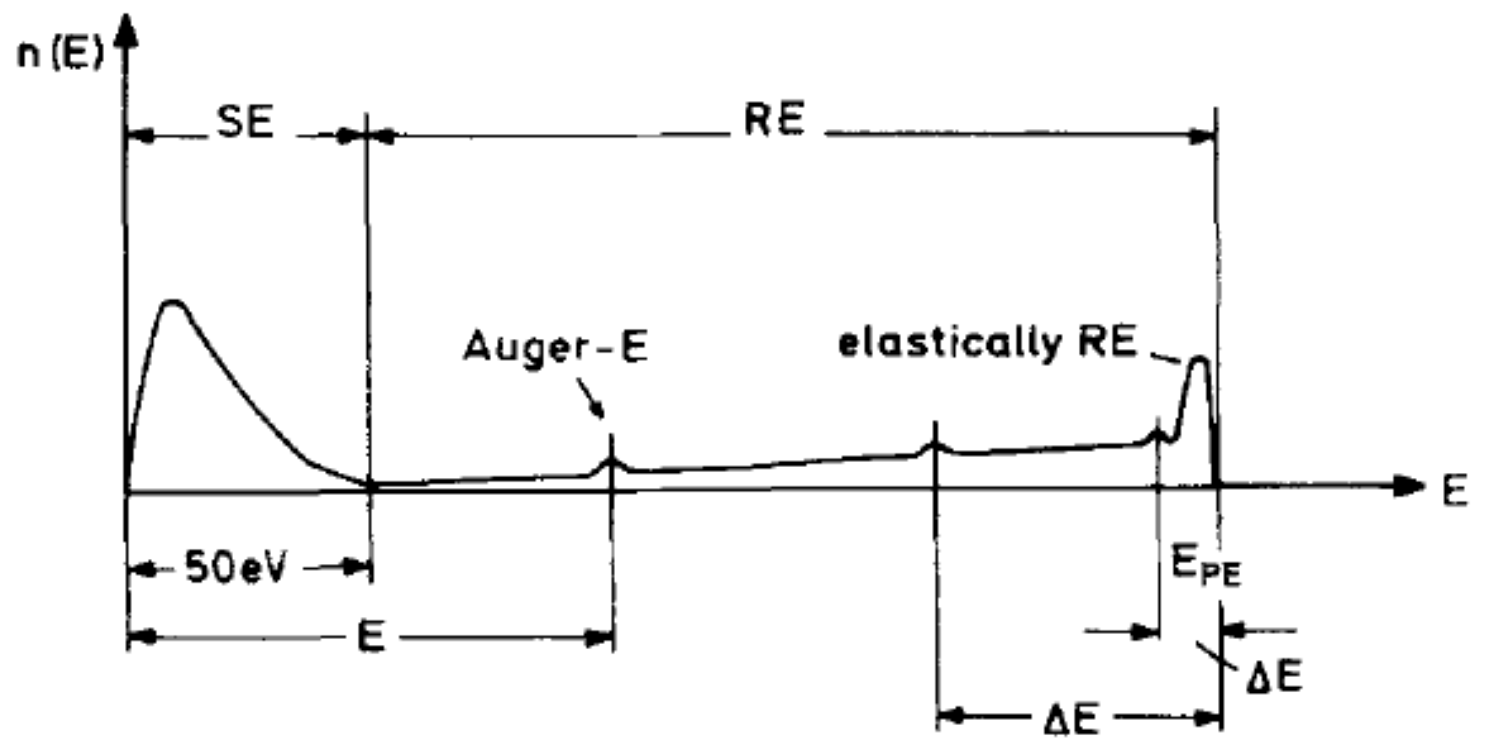

Figure 2.1: Schematic energy distribution of electrons which are emitted from a surface bombarded with $P E[20]$.

The secondary electron emission yield (SEY) is defined as the ratio of the number of secondaries leaving the surface to the number of incident primary electrons.

$$
\begin{gathered}
\delta=\frac{\text { Number of electrons leaving the surface }}{\text { Number of incident electrons }} \\
\delta=\frac{I_{C}}{I_{P}}=\frac{I_{P}-I_{S}}{I_{P}}=1-\frac{I_{S}}{I_{P}}
\end{gathered}
$$

$I_{P}$ is the primary current (the current leaving the electron gun and impinging on the surface of the sample) and $I_{S}$ is the total current measured on the sample flowing to ground $\left(I_{P}=I_{S}+I_{C}\right)$. 
$\mathrm{I}_{\mathrm{C}}$ is the current leaving the sample. It is the sum of the true secondaries and the elastically backscattered electrons.

The secondary electron emission is described through the yield $\delta\left(E_{0}, \theta_{0}\right)$ and its dependence on the emitted energy. $E_{0}$ and $\theta_{0}$ are the incident kinetic energy of the primary electron and its angle relative to the normal to the surface at the point of impact.

The form of a secondary electron yield curve as a function of primary electron energy is like in the Figure 2.2. When the yield is greater than one, more electrons are leaving the surface than the number of incident primary electrons.

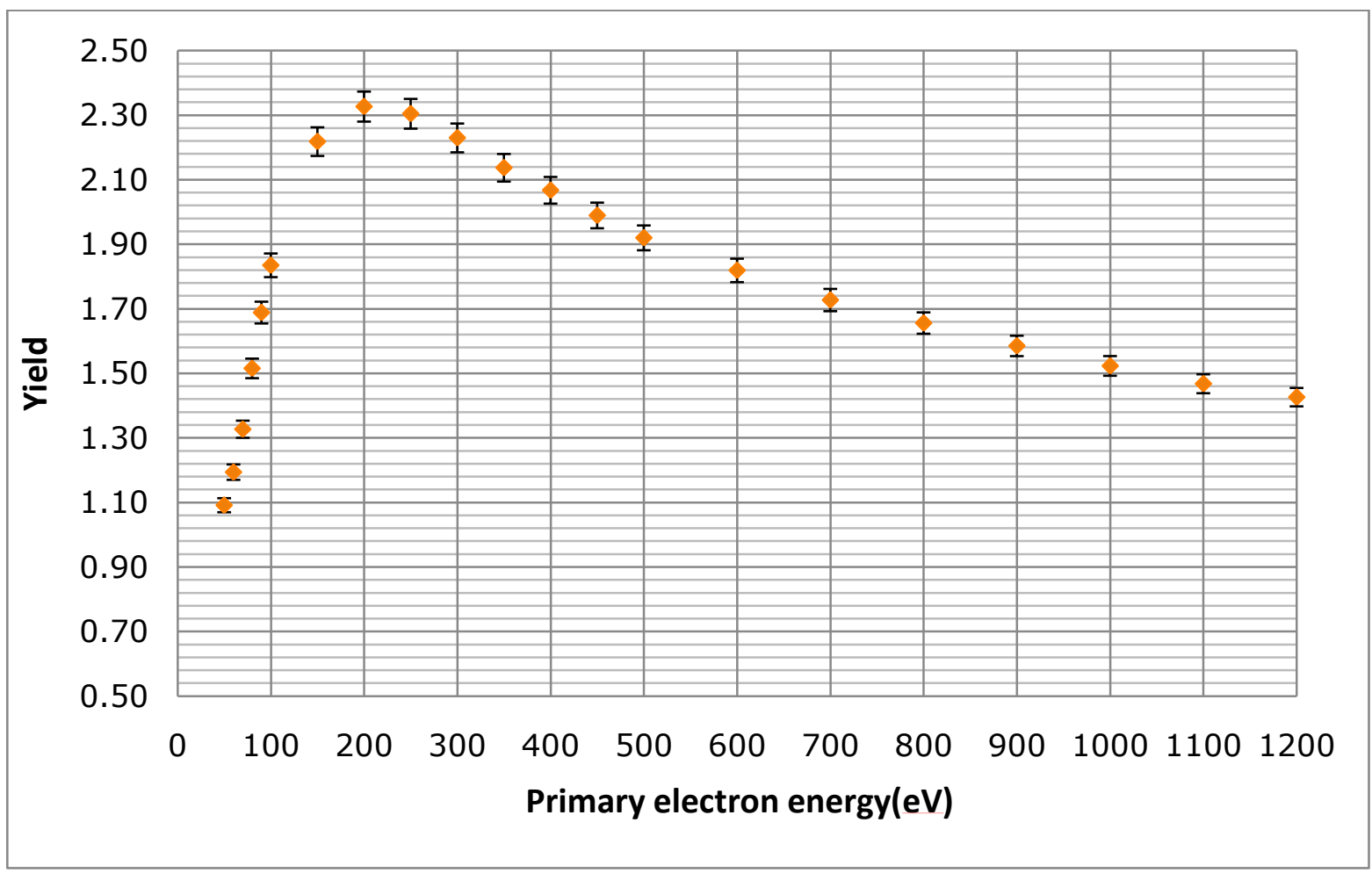

Figure 2.2: The SEY of an air exposed, polycrystalline copper as a function of the primary electron energy.

The secondary electron yield of copper in the as received state increases with the increasing energy of primary electrons and it reaches a maximum at energy of around $250 \mathrm{eV}\left(\mathrm{E}_{\max }\right)$. After reaching the maximum it starts to decrease with the energy. This is a general behaviour, which is found for most materials.

Primary electrons with high energies can penetrate deep into the target and lose most of their energies in regions where the liberated secondary electrons are not able to escape. With decreasing possibility to escape, the SEY starts to decrease (Figure 2.3). Secondary electrons generated at more than a certain distance, called the maximum escape depth $\varepsilon$, under the surface cannot escape from the specimen. 
With decreasing energy of the primary electrons, the penetration depth of the primaries decreases, therefore the secondary electrons are generated in a regime closer to the surface. The possibility to escape the surface increases and so does the yield. On the other hand, primary electrons with very low energies can only liberate few secondary electrons because the amount of available energy is low.

Secondary electrons originate in the case of metals from a layer of the order of $5-20 \AA$ below the surface and in the case of semiconductors and insulators slightly deeper due to the larger inelastic mean free paths in insulators [21].

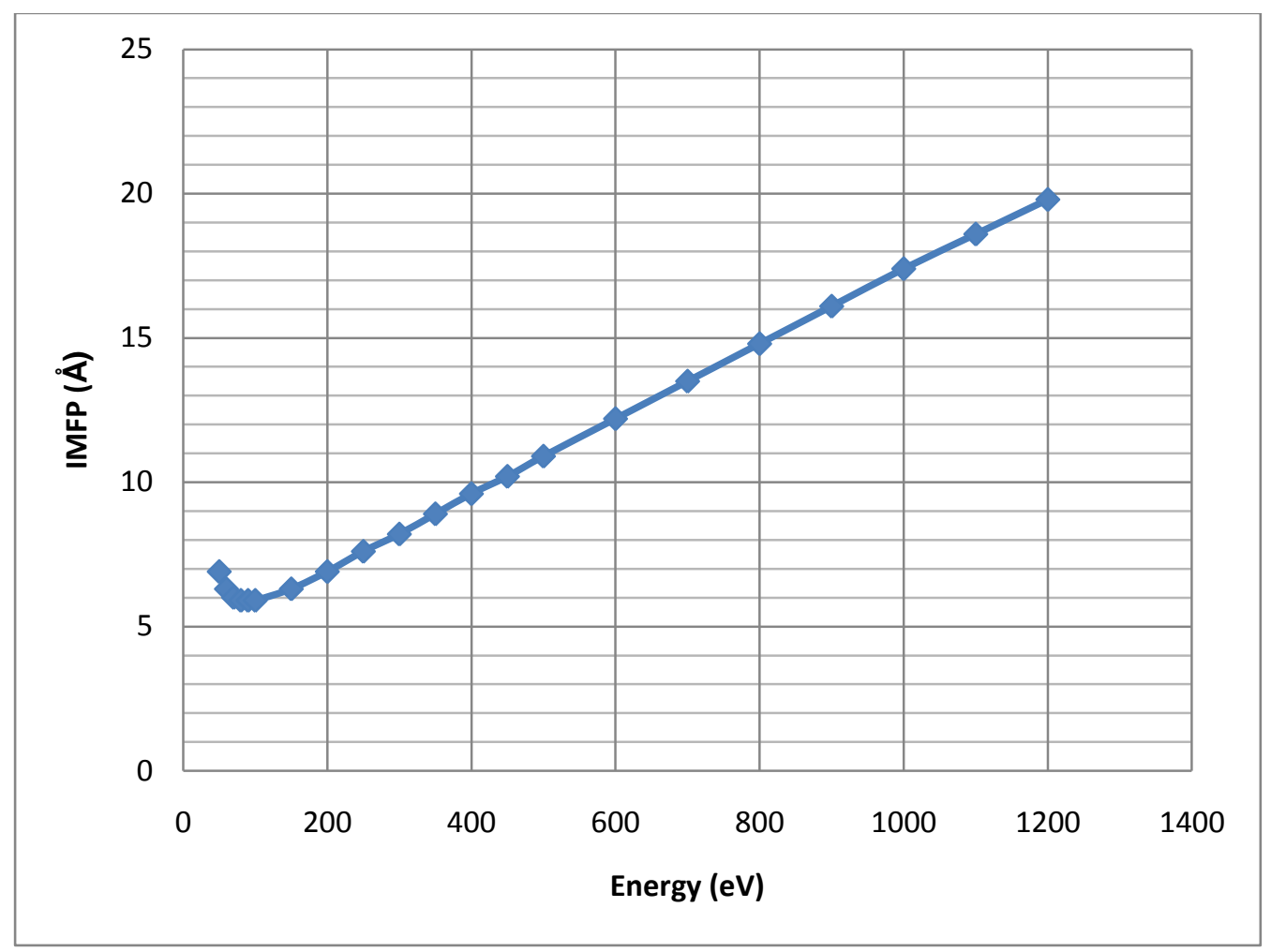

Figure 2.3: Inelastic mean free path of electrons in copper as a function of the kinetic energy [22].

In a simplified model the secondary electron yield can be written like following [20]:

$$
\delta=\int n\left(x, E_{P E}\right) f(x) d x,
$$

where $n\left(x, E_{P E}\right)$ is the number of secondary electrons produced in a distance $x$ from the surface through a primary electron at an energy of $E_{P E}$ and $f(x)$ is the probability that the secondary electrons can escape from the surface.

We can assume, that $\mathrm{n}$ is proportional to the average energy loss in the target:

$$
n\left(x, E_{P E}\right)=-\frac{1}{\varepsilon} \frac{d E}{d x}
$$


where $\varepsilon$ is the average energy per secondary electron excited at a distance $x$ from the surface. The probability for secondary electrons to escape is given by:

$$
f(x)=B e^{-x} / \lambda, B<1
$$

where $\lambda$ is the mean electron escape depth $[23,24]$.

As Young [25] assumed, based on the penetration data of electrons in $\mathrm{Al}_{2} \mathrm{O}_{3}$, the energy dissipation of primary electrons within the material is approximately constant:

$$
-\frac{d E}{d x}=E_{P E} /_{R}
$$

$R$ is the electron range and Young proposed a formula which was in close agreement with Bethe's theory prediction at low electron energies:

$$
\frac{R}{n m}=\frac{1.15 \cdot 10^{5}}{\rho\left(k g / m^{3}\right)}\left(\frac{E_{P E}}{k e V}\right)^{1.35}
$$

We can calculate the secondary electron yield by using the formulas above as follows:

$$
\begin{gathered}
\delta=\int_{0}^{\infty} \frac{B}{\varepsilon} \frac{E_{P E}}{R} e^{-x / \lambda} d x, \\
\delta\left(E_{P E}\right)=B \frac{E_{P E}}{\varepsilon} \frac{\lambda}{R}\left(1-e^{-R / \lambda}\right) .
\end{gathered}
$$

\subsubsection{Incidence angle dependence of secondary electron emission}

If the primary electron beam strikes on the surface with an angle $\theta$ to the surface normal, it will interact with the electrons at a mean depth of $\lambda \cdot \cos \theta$ instead of at the larger mean depth $\lambda$, which is the mean depth for perpendicular incident primary electrons. The secondaries, which get released at depth $\lambda \cdot \cos \theta$ have a shorter path to reach the surface, hence fewer of them get absorbed and so more secondaries can escape the surface. This means that the SEY yield increases with increasing angle $\theta$. 


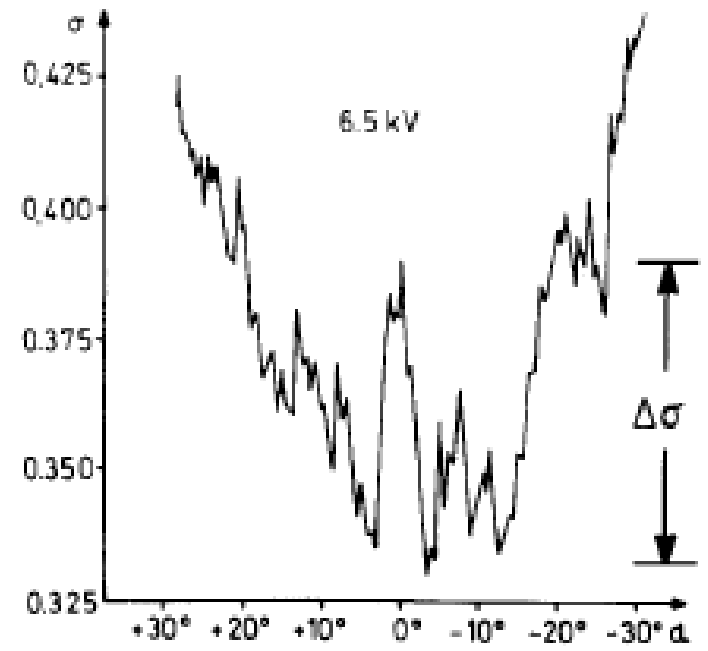

Figure 2.4: Dependence of $\sigma=\delta+\eta$ ( $\delta=$ number of true secondaries/number of primaries, $\eta=$ number of backscattering/number of primaries) on the angle of incident for a Si(111) face [26].

\subsubsection{Influence of the surface roughness on the SEY}

In general surface roughness can affect the physical processes in secondary electron emission in two ways. One way is that the surface roughness causes a dispersion of the incident angle of primary electrons. As long as the normal incidence on the macroscopic surface is considered, this effect results in larger electron yield in comparison with that for a flat surface, because of an increase in the yield with inclined incidence. The other effect is the re-entrance of electrons emitted from the surface into another part of the material. Secondary electrons, generated on a rough surface are facing other microstructure on their way to the surface and hence can lose part of their energy or even can get adsorbed before they can escape the surface. This effect causes a lower yield for rough surfaces than for flat surfaces.

The effect of surface roughness is simulated by [27], where it is to see that the SEY and the backscattering coefficient varies according to the height and width of the roughness. Figure 2.5 shows the model structure for surface roughness simulation and Figure 2.6 shows the variation of the SEY $\delta$ and the backscattering coefficient $\eta$ of aluminium for normal incidence as a function of $\mathrm{H} / \mathrm{W}$.

Figure 2.7 shows the influence of the surface roughness on SEY. 


\section{Chapter 2- Principles of secondary electron emission}

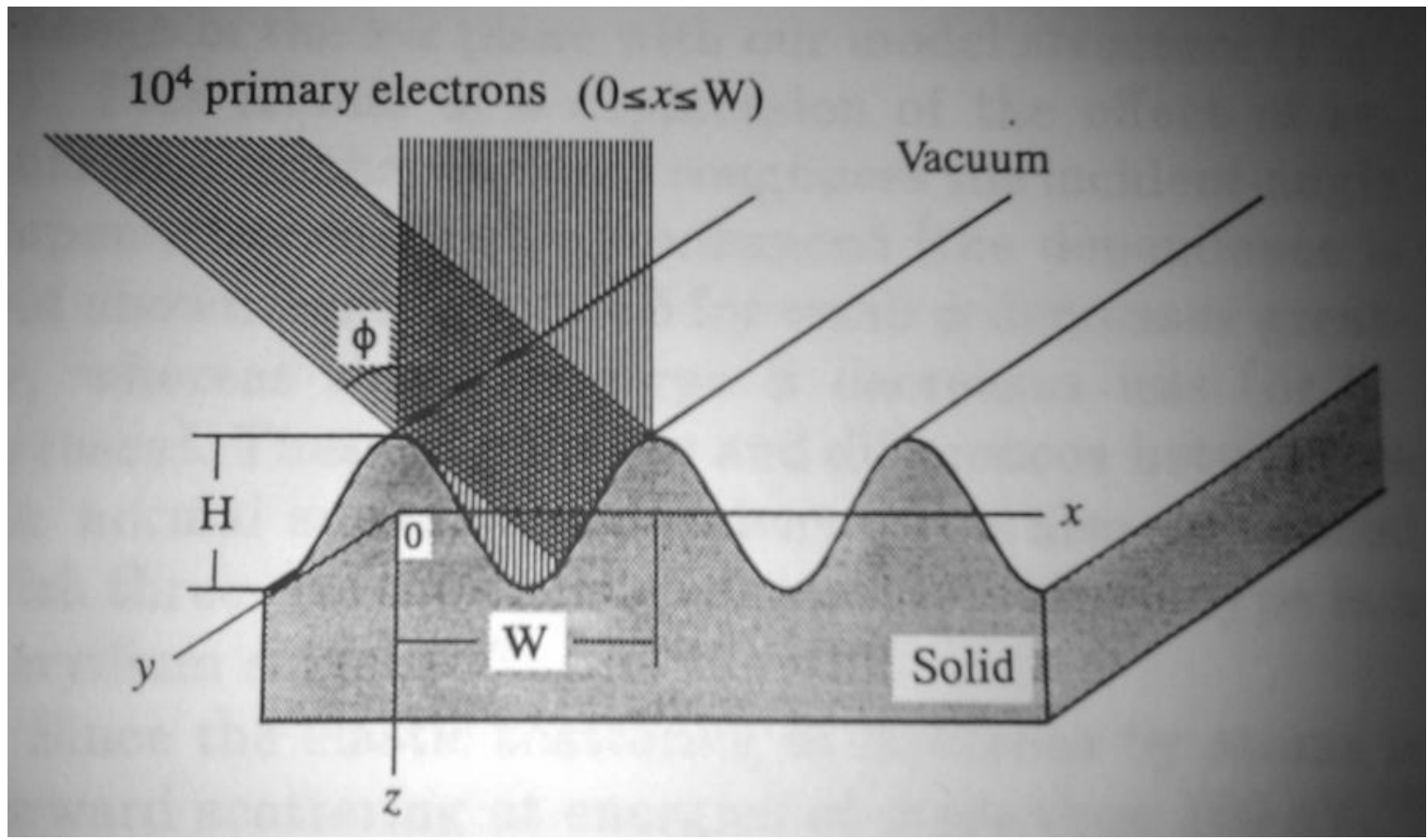

Figure 2.5: Model of ripple structure for surface roughness [27].

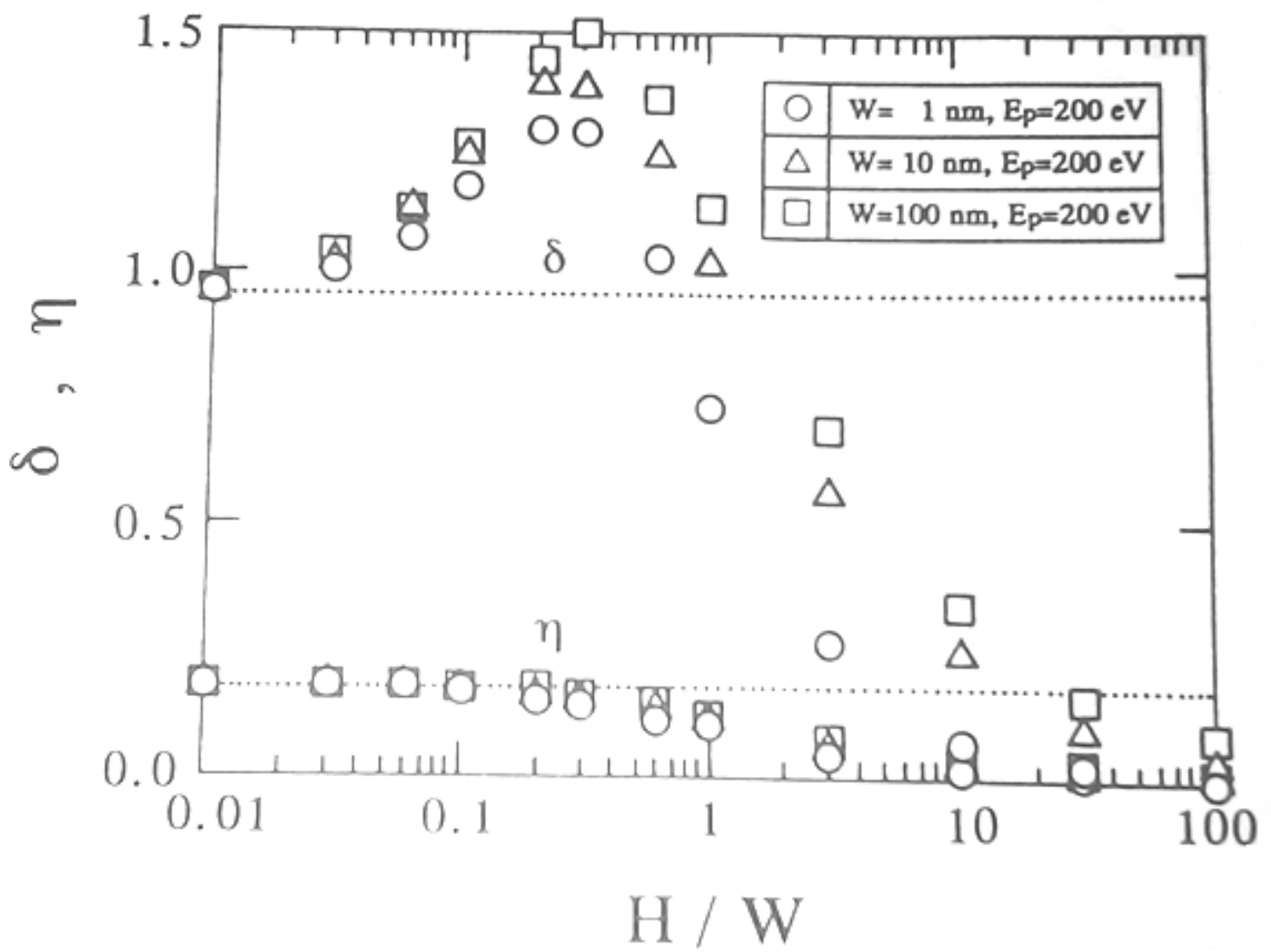

Figure 2.6: Variations of SEY $\delta$ and backscattering coefficient $\eta$ of aluminium for normal incidence $\left(\theta=0^{\circ}\right)$ as a function of $H / W$ for surface roughness, for different width $W$. The dotted lines are the calculated $\delta$ and $\eta$ for a flat surface [27]. 

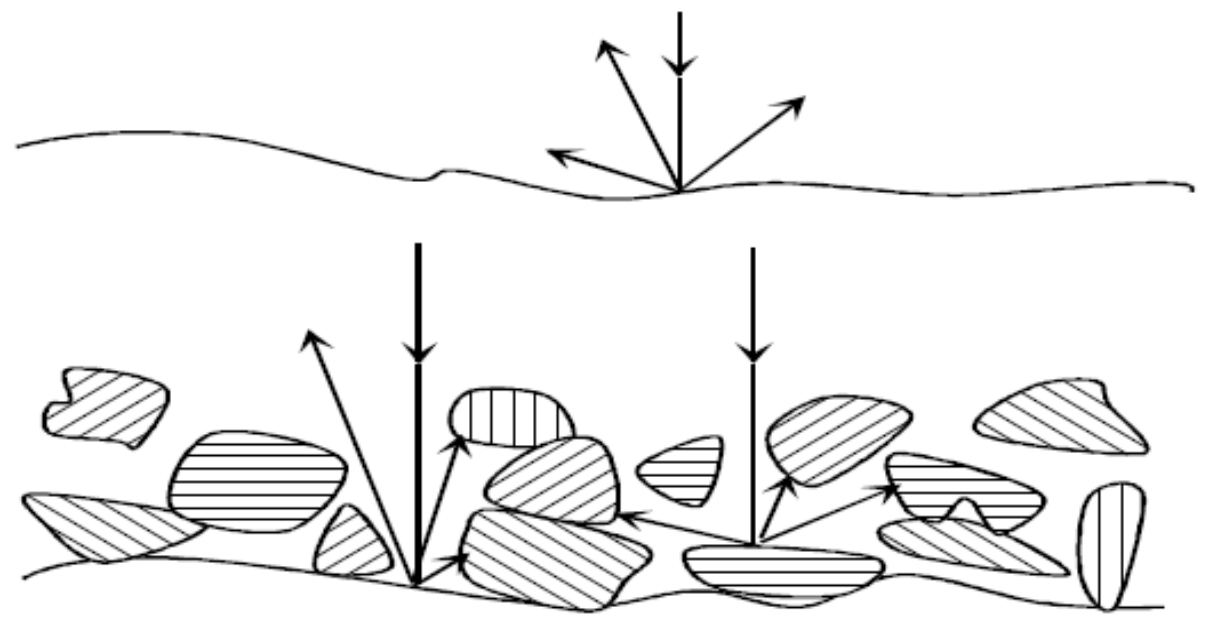

Figure 2.7: Influence of the surface roughness on the SEY. The probability for the electrons to escape is higher from a smooth surface[28].

\subsubsection{The effect of adsorbed layers and air exposure on the SEY}

The maximum escape depth of secondary electrons from metals is estimated as 10 atomic layers [29]. Assuming a thickness of $1 \AA$ for one atomic layer, the emitted electrons origin from the upper $1 \mathrm{~nm}$ of the solid. The probability for the low energetic secondary electrons to pass through a condensate layer of adsorbed gases is higher than through a metal. The escape depth of secondary electrons in such layers can reach about 100 monolayers [29].

According to the [30], the maximum yield $\delta_{\max }$ of copper is below 2 at an energy $E_{\max }$ at around $200-250 \mathrm{eV}$. In case of adsorbed layers of different gases, this yield changes rapidly. For example in case of $\mathrm{Ne}$, depending on the coverage, the yield increases. It reaches a value of around 5.25 (at $E_{\max } \approx 500 \mathrm{eV}$ ) for $\mathrm{D}=60 \mathrm{Ne}$ monolayers and it reaches a value of app. 30.8 (at $E_{\max } \approx 2400 \mathrm{eV}$ ) for $\mathrm{D}=1000$ monolayers of $\mathrm{Ne}$. Also with other rare gases the yield reaches very high values. The explanation for such behaviour is the large escape probability of the secondary electrons and the large attenuation length for the secondary electrons. The adsorbate layer may also change the secondary electron yield due to the change of the work function. According to [31], the deposition of 0.5 monolayer of Cs increased the yield of copper from 1.54 to 3.40. Alkali metals have a lower work function than that of the transition metal; therefore the secondary electrons can leave the surface easier so the yield increases.

Air exposure is changing the yield of the materials as well. In Figure 2.8 the yield of glow discharged copper after 5 minutes and 6 days of air exposure are shown. Exposures of a 
few minutes decrease the yield from 1.4 to about 1.2 and the energy at the maximum yield is shifted as well. With more air exposure the yield increases [28].

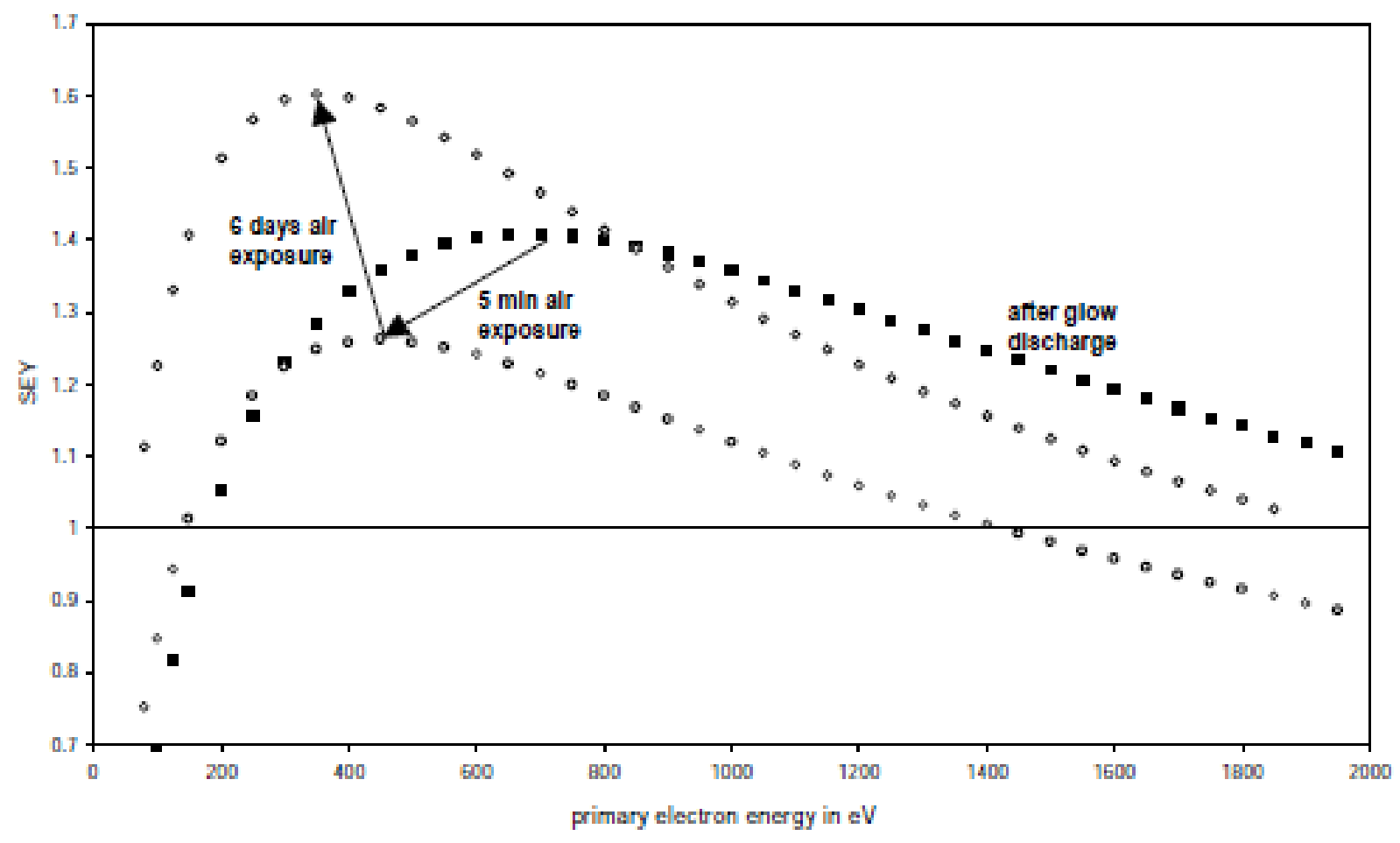

Figure 2.8: SEY of glow discharged copper, after 5 minutes and 6 days of air exposure inside the vacuum vessel as a function of primary electron energy[28].

\subsubsection{Conditioning (beam scrubbing)}

Secondary electron yield of technical metals depends strongly on the surface and on the composition of the oxide layer, as well as the condensed gas layers. The effect of dosing of a surface with electrons or photons decreases the secondary electron yield to a value which can be lower than the one of the base material. This process is called conditioning. The physical process, which causes the reduction of the yield, can be attributed in first position to the cleaning of the surface and in second position to the formation of carbon rich layer on the top surface [32]. Carbon is known to have a low secondary electron yield. There is no indication that the final secondary electron yield after conditioning depends on the base material. Conditioned surface is very sensitive to the air exposure but the reconditioning takes less time.

Conditioning is an important phenomenon in LHC because during operation of RF power components a gradual 'conditioning' occurs. Therefore investigations about this phenomenon are important for accelerator technology.

The required dose for conditioning via electron bombardment is very large compared to the electron dose during SEY measurements. With the electron dose of about $10^{-3} \mathrm{C} / \mathrm{mm}^{2} \mathrm{SEY}$ of 
copper colaminated on stainless steel can be reduced from 2.2 to 1.2. Figure 2.9 shows the variation of the secondary electron yield of as received copper and argon glow discharged copper electron bombardment and the SEY variation of the conditioned copper under argon ion bombardment. Figure 2.10 shows the effect of the beam scrubbing on the SEY as a function of the primary electron energy.

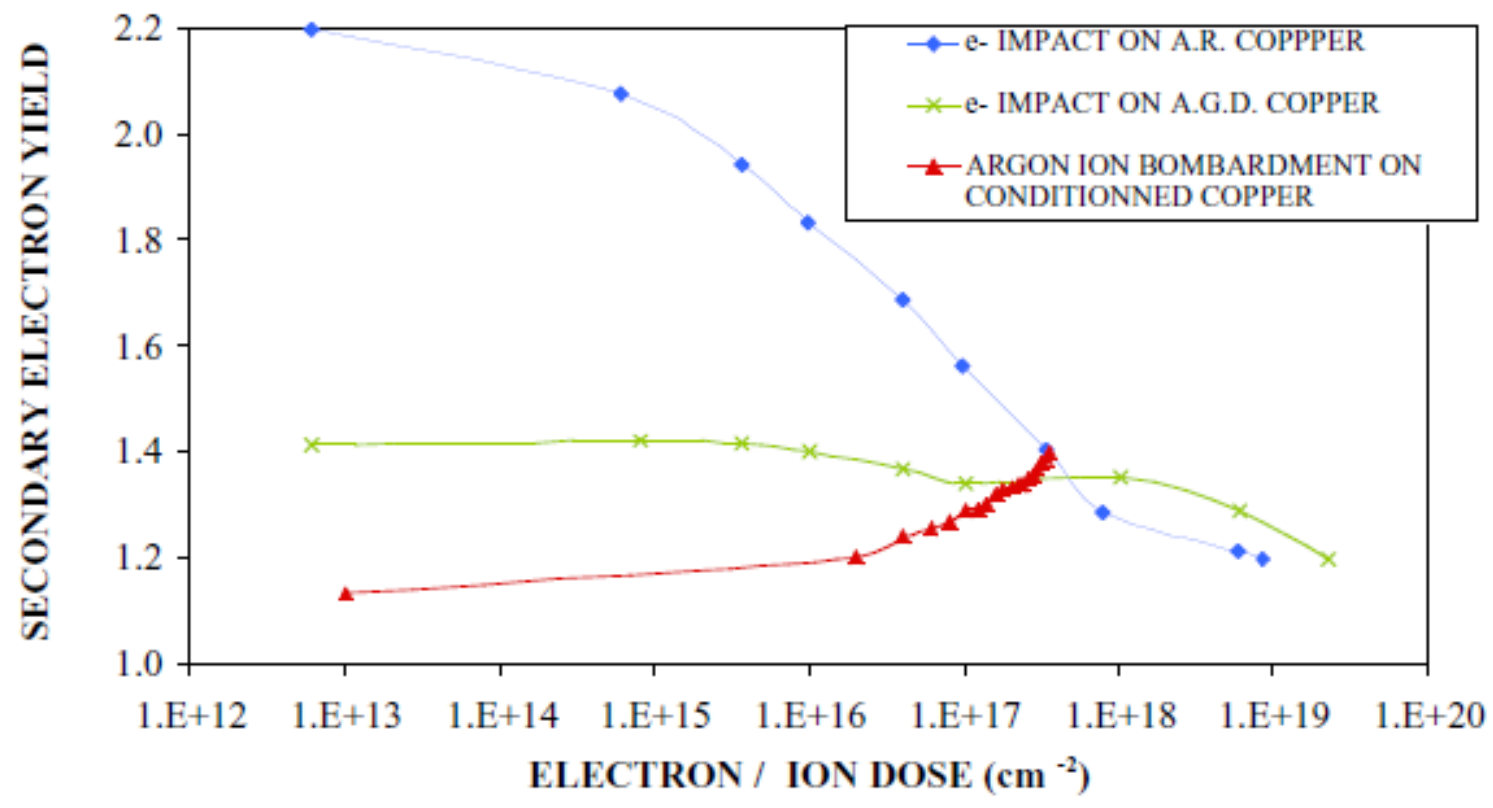

Figure 2.9: The variation of the secondary electron yield under electron/ion bombardment[32].

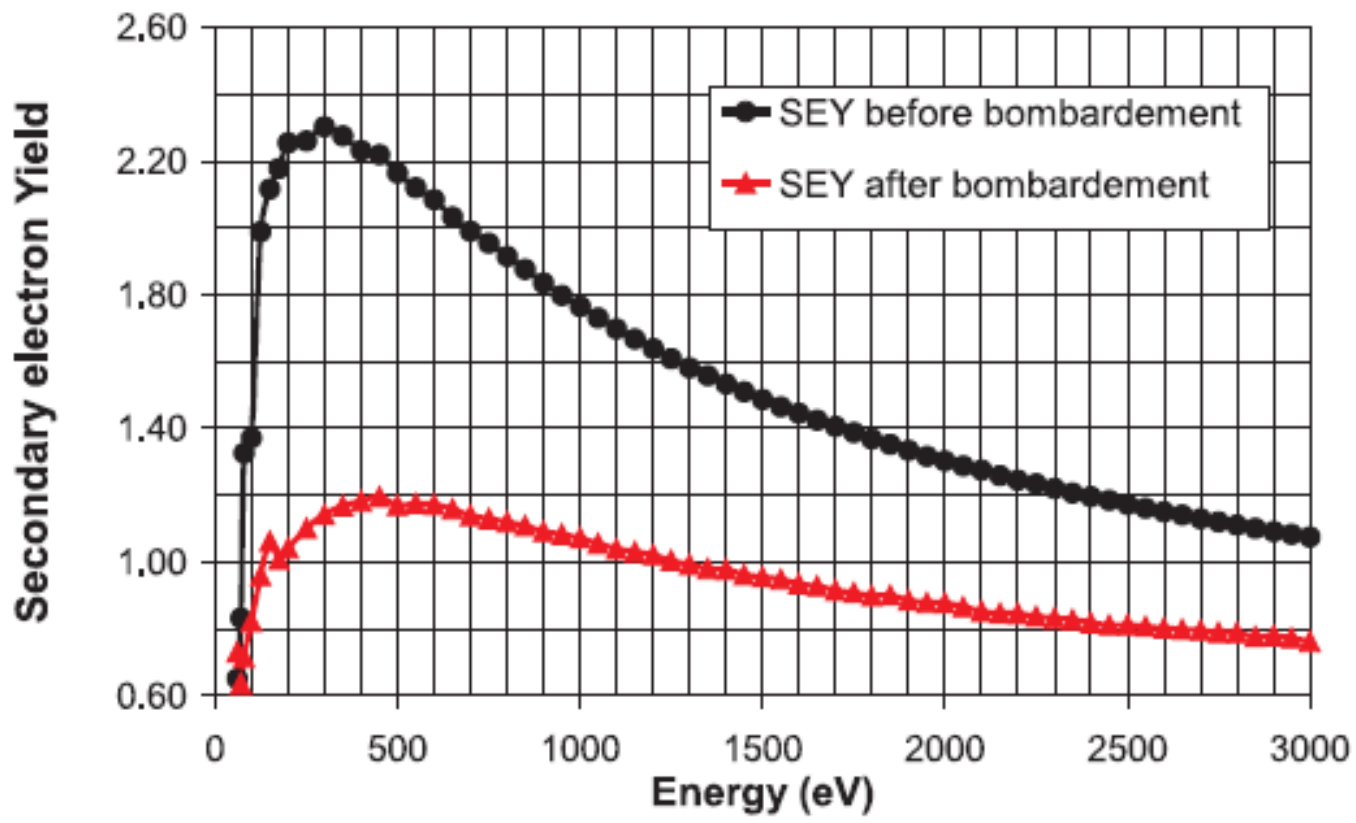

Figure 2.10: SEY as a function of the primary electron energy for a copper sample colaminated on stainless steel before and after beam scrubbing. Electrons with $500 \mathrm{eV}$ corresponding to a dose of $5 \cdot 10^{-3} \mathrm{C} / \mathrm{mm}^{2}$ [33]. 


\subsection{Electron cloud build-up}

Electron cloud effect, which involves the interaction between high-energy beams and lowenergy electrons produced in the vacuum chamber, is a limitation for the performance of high energy accelerators.

Due to the close bunch spacing of $25 \mathrm{~ns}$, an important number of electrons are generated in the LHC beam pipe by different production mechanism or a combination thereof. Figure 2.11 shows the electron cloud build-up mechanism in LHC [34].

In LHC at injection energy the main source of the primary electrons is the residual gas ionization. The designed density of hydrogen in LHC is about $10^{15}$ molecules $/ \mathrm{m}^{3}$ and of carbon monoxide is $1,3 \cdot 10^{13}$ molecules $/ \mathrm{m}^{3}$, which corresponds for a beam current of $0,7 \mathrm{~A}$ to a production rate of primary electrons with a typical value of[35]:

$$
\frac{d^{2} \lambda_{e}}{d s d t} \approx 2 \cdot 10^{11} e^{-} m^{-1} s^{-1}
$$

At $7 \mathrm{TeV}$ most of the primary electrons are generated by synchrotron radiation and photoemission. The number of photons emitted by a charged particle of charge Z.e per unit path length and per unit energy interval, or equivalently to $\lambda$, of the photons is equal to [36]

$$
\frac{d N^{2}}{d x d \lambda}=\frac{2 \pi \alpha Z^{2}}{\lambda^{2}} \cdot\left(1-\frac{1}{\beta^{2} \cdot n^{2}(\lambda)}\right)
$$

where $\alpha$ is the fine-structure constant, and $n$ is the refraction index $(\beta=v / c)$. In case of LHC at $7 \mathrm{TeV}$, the amount of photons produced per $14.2 \mathrm{~m}$ long bend and per proton bunch is about $3.7 \cdot 10^{10}[37]$.

In LHC the typical photoemission yield at energies of $10-100 \mathrm{eV}$ is 0,1 [38]. Following the reference [39], the beam pipe material for those photon wavelengths with an incident angle of $2^{\circ}$ relative to the surface has a reflectivity of $90 \%$, which allows us to assume that on average a photon is either reflected or converts into a photoelectron, so that the final number of electrons is approximately equal to the number of radiated photons. Following that 
assumption we can consider that every photon is reflected up to 10 times before it creates a photoelectron[37].

A photoelectron produced by a synchrotron radiation of the bunch head is accelerated by the field of the beam and it receives a maximum transverse momentum of [37]

$$
\Delta p_{e} \approx \frac{2 N_{b} r_{e} c m_{e}}{r_{p i p e}} \quad r_{e}=\frac{1}{4 \pi \varepsilon_{0}} \frac{e^{2}}{m_{e} c^{2}}
$$

$r_{\mathrm{e}} \ldots$...lassical electron radius

c...speed of light

$r_{\text {pipe....radius of the beam pipe }}$

$\mathrm{m}_{\mathrm{e}} \ldots$ electron mass

$N_{b} \ldots$ number of particles/bunch

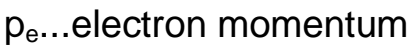

Considering the LHC parameters the accelerated photoelectrons have energy of approximately $200 \mathrm{eV}$, which corresponds a wall-to-wall time of flight of about $5 \mathrm{~ns}$. Most of the primary electrons hit the wall, before the next bunch arrives. The secondary electrons generated by the electrons of $200 \mathrm{eV}$ have of a factor 5-10 lower velocities. If the effective secondary electron yield (SEY) of the chamber is larger than unity, the number of electrons grows rapidly and leads to a high electron cloud density. If the traversal time of the electrons is comparable to the time interval between two bunches, a resonance condition is reached and the number of electrons grows exponentially. (Beam induced multipacting). Thus without an electron space-charge force, the number of electrons would increase indefinitely[37].

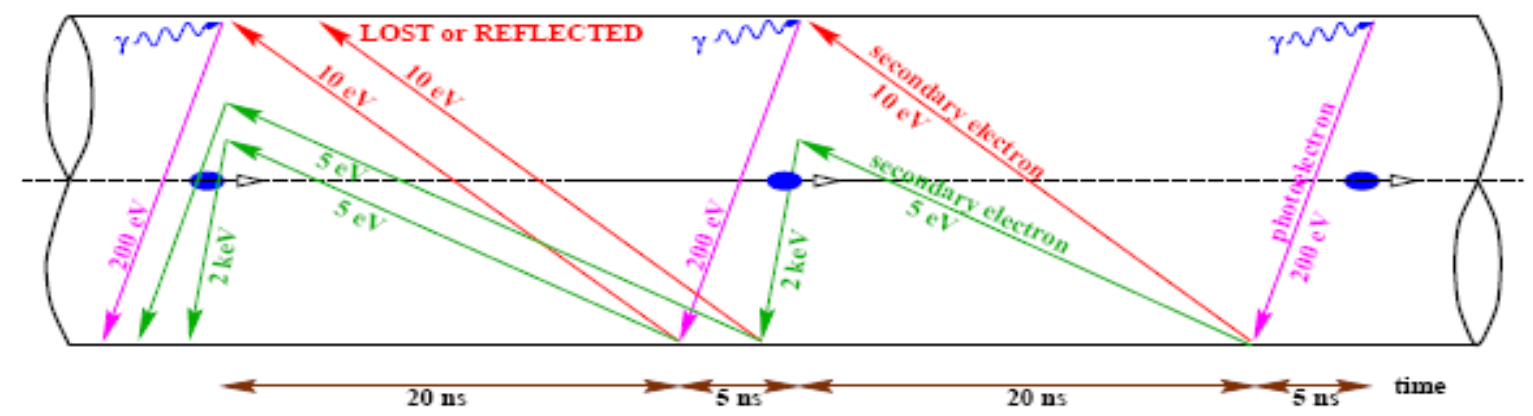

Figure 2.11. Electron cloud build-up in LHC (courts F.Ruggiero). 
The electron cloud density depends on characteristics of the circulating beam (bunch length, magnetic field, charge and spacing), the chamber geometry and the secondary electron yield (SEY) of the wall from which the electrons are generated.

The strength and the configuration of the magnetic field are essential for the dynamics of the electrons. In inhomogeneous magnetic fields like quadrupoles, electron trapping can occur, which enhance the electron cloud effect. In dipole fields, which are $80 \%$ of LHC, the electrons undergo only a vertical motion during their life time, because the cyclotron period is much shorter than the bunch length and they do not receive any horizontal force by the bunch.

\subsubsection{Electron cloud effects}

The electron cloud can provoke a number of undesirable effects. It leads to a limitation of the beam vacuum by several orders of magnitude, to degradation of the beam quality, due to generation of collective instabilities (coupled bunch and single bunch), to beam size increases, to fast or slow beam losses, It also cause noise in beam diagnostics, beam position monitors and feedback pick-ups, due to unequal electron bombardment. Electron cloud can also give rise to a heat load inside the cold superconducting magnets.

\section{a)Single bunch instability}

Short-range head-tail wake field arising from electron cloud may amplify any initial small deformation in the bunch and cause single-bunch instability. That kind of instability causes a vertical emittance blow-up. A proton or positron bunch interacts with the electron cloud during its passage. If the bunch enters the electron cloud with a slight offset on the head (Figure 2.12), the electrons accumulate around the beam centre (pinch effect). This new electron distribution will kick the following bunch particles toward the higher density region. Therefore the motion of the head will be transmitted and amplified at the tail of the bunch [40].

Without synchrotron motion, the resulting instability resembles the beam breakup in a LINAC. If synchrotron motion is included, the instability becomes similar to a regular transverse mode coupling instability (TMCI) [41, 42]. 


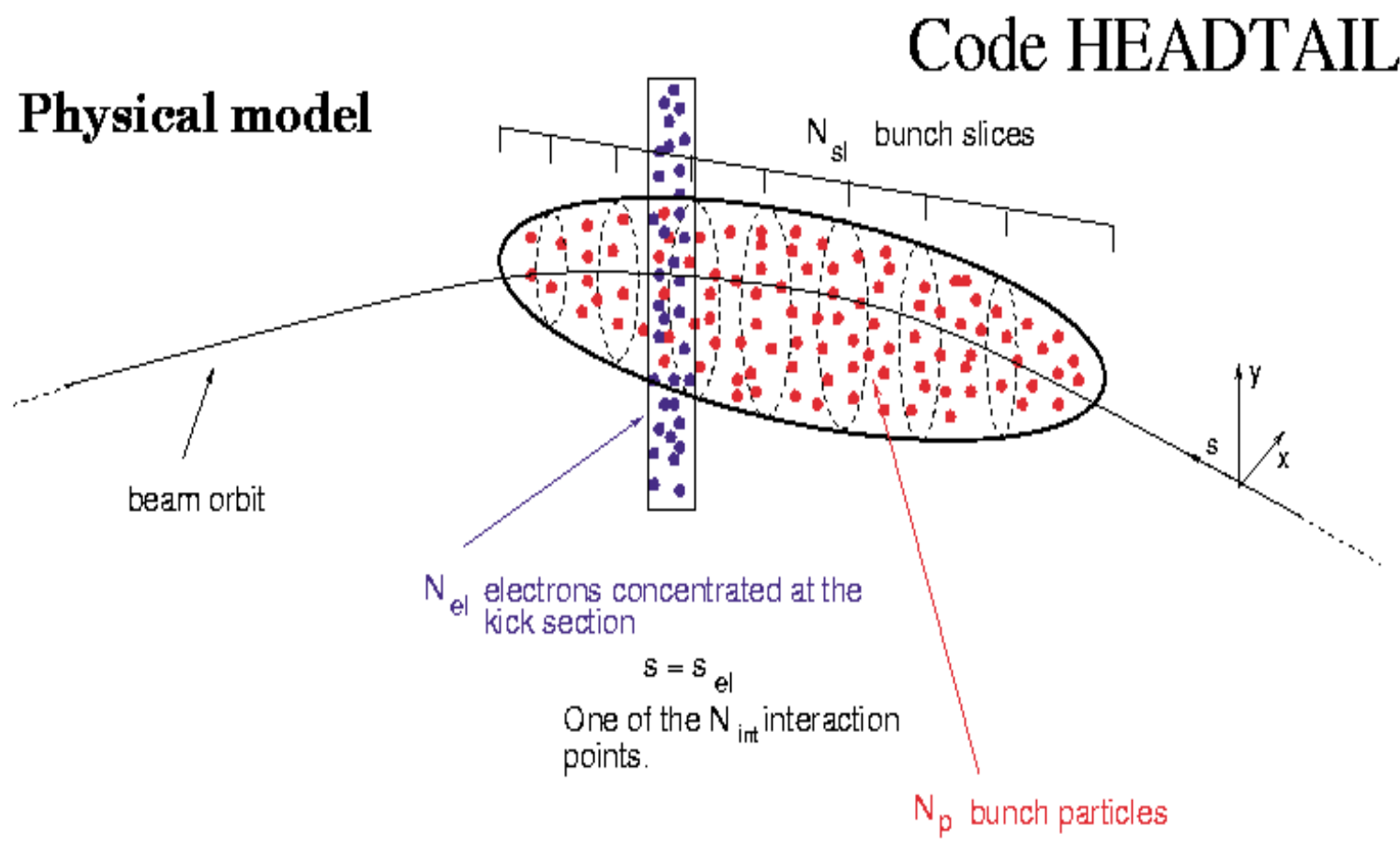

Figure 2.12: Simulation of the single bunch instability [40].

\section{b) Multiple bunch instability}

If a bunch is sent through the electron-cloud offset with respect to the other bunches, it will perturb the electron-cloud distribution and the next bunch will receive an additional deflection caused by this perturbation. This instability called multibunch instability is expected to be very weak for the LHC at collision energy (the rise time is on the order of 1s) but it can be a concern at injection energy and in the SPS.

\section{c) Heat load provoked by electron cloud}

Another important effect of the electron cloud on the LHC machine is the heat load inside the cold superconducting magnets. If the heat load exceeds the limited cooling capacity at cryogenic temperature, it can lead to magnets transition into normal-conducting state ("quench"). In LHC to reach the required magnetic dipole field and the magnetic rigidity in the quadrupoles, a superconducting technology at $1.9 \mathrm{~K}$ is used [6]. To keep the heat load at minimum, a beam screen is placed inside the vacuum cold bore held at $1.9 \mathrm{~K}$. The temperature of the beam screen reaches $20 \mathrm{~K}$, while adsorbing the heat dissipated by the beam. In the "as received" state (not conditioned state), the assumption of arc heat load for a maximum yield of 1.9 is around $4 \mathrm{~W} / \mathrm{m}$ with half of the nominal bunch intensity 
$\left(0.5 \cdot 10^{11}\right.$ proton/bunch) [35]. This heat load can be reduced by using the beam itself to condition the chamber surface by electron bombardment (beam scrubbing).

As described above Electron Cloud Effect is an important aspect in high energy particle accelerators, which depends strongly on the SEY. There are several investigations about the SEY at room temperature although there are only few investigations about SEY at cryogenic temperatures and fewer about SEY as a function of physisorbed gases. The aim of this project is to cover up the lack in that field. 


\section{Introduction to the vacuum physics}

\subsection{Vacuum for SEY measurements}

It is necessary to perform the SEY measurements in ultrahigh vacuum conditions because it is important to have surfaces free of gases. The properties of the vacuum chamber wall and accordingly of the sample material are of decisive importance since below $10^{-3}$ mbar there will be more gas molecules on the surfaces than in the volume of chamber. If one assumes a monomolecular adsorbed layer on the inside wall of an evacuated sphere with 1 volume, then the ratio of the number of adsorbed particles to the number of free molecules in the volume will be as follows [43]:

at 1 mbar $10^{-2}$

at $10^{-6} \mathrm{mbar} \quad 10^{+4}$

at $10^{-11} \mathrm{mbar} \quad 10^{+9}$

For this reason the monolayer formation time is of importance. It is only a fraction of a second in case of high vacuum $\left(10^{-3}-10^{-7} \mathrm{mbar}\right)$ while in the ultrahigh vacuum $\left(10^{-7}\right.$ $10^{12} \mathrm{mbar}$ ) range it can extend over a period of minutes or hours. Clean surfaces inside the vacuum chamber can therefore only be achieved under ultrahigh vacuum conditions.

In addition at cryogenic temperatures to avoid that the rest gas molecules in the vacuum chamber get adsorbed on the cold sample surface, it is necessary to reach ultrahigh vacuum. 
For the measurements of SEY in a laboratory setup, to work in ultrahigh vacuum conditions is also important to produce a focused beam. The mean free path of electrons at atmospheric pressure is only $1 \mu \mathrm{m}$. At ultrahigh conditions it can reach kilometres. Finally elimination of oxygen prevents "burning out" of hot filaments as for the e-gun, residual gas analyzer and Bayard \& Alpert gauge.

\section{2 Kinetic theory of gases and the mean free path}

The velocity of the gas molecules, which are in constant movement, depends on the molar mass $\mathrm{M}$ and the temperature $\mathrm{T}$ only. The root-mean-square velocity $\mathrm{v}_{\mathrm{rms}}$ is given by [44]:

$$
v_{r m s}=\left(\frac{3 R T}{M}\right)^{\frac{1}{2}}=\left(\frac{3 k T}{m}\right)^{\frac{1}{2}}
$$

M...Molar mass

m...Mass

T...Temperature

\begin{tabular}{|c|c|c|c|c|c|c|c|c|c|c|c|c|c|}
\hline \multirow{2}{*}{$\sum^{\text {Gas }}$} & \multicolumn{13}{|c|}{$v_{\text {rms }}\left(\mathrm{m} \mathrm{s}^{-1}\right)$} \\
\hline & \multicolumn{5}{|c|}{ monoatomic } & \multicolumn{5}{|c|}{ diatomic } & \multicolumn{3}{|c|}{ polyatomic } \\
\hline 1 & $\mathrm{He}$ & $\mathrm{Ne}$ & $\mathrm{Ar}$ & $\mathrm{Kr}$ & $\mathrm{Xe}$ & $\mathrm{H}_{2}$ & $\mathrm{~N}_{2}$ & $\mathrm{CO}$ & $\mathrm{O}_{2}$ & Air & $\mathrm{NH}_{3}$ & $\mathrm{C}_{2} \mathrm{H}_{2}$ & $\mathrm{CO}_{2}$ \\
\hline 273 & 1305 & 581 & 413 & 285 & 228 & 1838 & 512 & 493 & 461 & 485 & 632 & 511 & 393 \\
\hline 296 & 1358 & 605 & 430 & 297 & 237 & 1914 & 533 & 514 & 480 & 505 & 659 & 533 & 410 \\
\hline
\end{tabular}

Table 3.1: The root-mean-square velocity $v_{\text {rms }}$ of different gas molecules at 0 and $23^{\circ} \mathrm{C}$ [45].

The kinetic energy of gases is given like following:

$$
E_{k i n}=\frac{3 k T}{2}
$$


Due to the collisions between the gas molecules, the velocity of gases has a wide distribution from about zero to very large values. This is described by the MaxwellBoltzmann law:

$$
(1 / n) d n / d v=(4 / \pi)^{1 / 2}(m / 2 k T)^{3 / 2} v^{2} \exp \left(-\left(m v^{2}\right) / 2 k T\right)
$$

$d n$ is the number of particles in the velocity range between $v$ and $v+d v$ [44].

The average distance between collisions for a gas molecule is called the mean free path $\lambda$. The mean free path can be taken as the length of the path divided by the number of collisions. It depends on the density of the gas and also on the size of the molecules.

$$
\lambda=1 /\left(\sqrt{2} \pi d^{2} n\right)=k T /\left(\sqrt{2} \pi d^{2} p\right)
$$

d...Molecular diameter in $\mathrm{m}$

n...Number of molecules per volume

p...Pressure in $\mathrm{Pa}$

The pressure range, which spans more than 16 powers of ten, is divided into five regimes. These are defined as follows:

\begin{tabular}{|c|c|c|}
\hline Rough vacuum (RV) & $1000-1 \mathrm{mbar}$ & $10^{5}-10^{2} \mathrm{~Pa}$ \\
\hline Medium vacuum (MV) & $1-10^{-3} \mathrm{mbar}$ & $10^{2}-10^{-1} \mathrm{~Pa}$ \\
\hline High vacuum (HV) & $10^{-3}-10^{-7} \mathrm{mbar}$ & $10^{-1}-10^{-5} \mathrm{~Pa}$ \\
\hline Ultrahigh vacuum (UHV) & $10^{-7}-10^{-12} \mathrm{mbar}$ & $10^{-5}-10^{-10} \mathrm{~Pa}$ \\
\hline Extreme high vacuum (XHV) & $10^{-12}-10^{-14} \mathrm{mbar}$ & $10^{-10}-10^{-12} \mathrm{~Pa}$ \\
\hline
\end{tabular}

Table 3.2: Pressure ranges.

\begin{tabular}{|c|c|c|}
\hline Pressure (mbar) & Mean free path (m) & Number of molecules $\left(\mathbf{m}^{-3}\right)$ \\
\hline $10^{3}$ & $64^{*} 10^{-9}$ & $2.5^{\star} 10^{25}$ \\
\hline 1 & $64^{*} 10^{-6}$ & $2.5^{\star} 10^{22}$ \\
\hline $10^{-3}$ & $64^{\star} 10^{-3}$ & $2.5^{\star} 10^{19}$ \\
\hline $10^{-6}$ & 64 & $2.5^{\star} 10^{16}$ \\
\hline $10^{-10}$ & $64^{\star} 10^{3}$ & $2.5^{\star} 10^{12}$ \\
\hline
\end{tabular}

Table 3.3: Pressure ranges, mean free paths, and density for nitrogen at room temperature. 
With the change of pressure further physical properties change as well. While the thermal conductivity and internal friction of gases in the medium vacuum range are highly sensitive to pressure, in the rough and high vacuum regimes these properties are still proportional to pressure, but in general negligibly small.

To achieve different vacuum regimes different vacuum pumps are needed and also different vacuum gauges are required. The vacuum pumps and vacuum gauges used in the system for SEY measurements will be explained detailed in chapter 4 .

\section{3 Conductance in vacuum systems}

The conductance of a 'passive' vacuum component (e.g. tube, nipple, elbow, tee, valve, etc.) is a measure of that component's ability to transmit gas molecules from end-to-end in some given time. High conductance is of paramount importance in achieving rapid chamber pump down times and low base pressures. The conductance of a system is one of the parameters to calculate the gas flow in that system. Gas flow due to pressure difference can be comparable with the flow of heat or electricity in conductor, caused by a voltage difference. The flow rate (also called "throughput") $Q$ is given like following:

$$
Q=\frac{\left(p_{1}-p_{2}\right)}{Z}=\frac{\Delta p}{Z}=C \Delta p
$$

$\mathrm{C}=1 / \mathrm{Z}[\mathrm{l} / \mathrm{s}]$ is called conductance of a pipe.

In analogy with the thermal and electric circuits, we find that the overall conductance $\mathrm{C}_{0}$ for individual flow elements in series is given by

$$
\frac{1}{C_{0}}=\sum_{i}\left(\frac{1}{C_{i}}\right)
$$

For individual flow elements in parallel, the overall conductance is given by

$$
C_{0}=\sum_{i} C_{i}
$$


The gas flow is subdivided in 3 regimes: Viscous flow, intermediate flow and molecular flow. Viscous flow is again divided into 2 regimes called turbulent flow and laminar flow. The regimes are determined by the Knudsen number and the Reynolds number.

The limits between laminar, intermediate and molecular flow is defined by the Knudsen number and the limit between turbulent and laminar flow is defined by the Reynolds number.

The Reynolds number is expressed by

$$
R=\frac{d v \rho}{\eta}
$$

v....Gas velocity

p...Density of the gas at the temperature of the flow

$\eta \ldots$ Viscosity of the gas at the temperature of the flow

d... Diameter of the pipe or the characteristic size of the vacuum component

The Knudsen number is defined as followed:

$$
K=\frac{\lambda}{d}
$$

\section{$\lambda$...Mean free path}

1) Viscous or continuum flow:

This will be found almost exclusively in the rough vacuum range. This type of flow is determined by the interactions of the molecules. It is divided in two groups:

a) Turbulent flow: $\mathrm{R}>2200, \mathrm{~K}<0.01$

b) Laminar flow: $R<1200, K<0.01$

2) Intermediate or Knudsen flow: $1.0>\mathrm{K}>0.01$

The transitional range between viscous flow and molecular flow is called Knudsen flow. It is prevalent in the medium vacuum range. 
3) Molecular flow: $\mathrm{K}>1.0$

This will be found in the high and ultrahigh vacuum ranges. In performing of the experiments described in this thesis, the gas flow was in the molecular flow regime, which is explained more in details below.

\section{Molecular flow $\left(\mathrm{p}<10^{-3} \mathrm{mbar}\right)$ :}

If the mean free path of the gas molecules is large compared to the dimensions of the pipe, the flow of the gas is called molecular flow. This occurs at low pressures, because most collisions are with the walls of the pipe rather than other gas molecules [43]. As a result of reflection a gas particle can move in any arbitrary direction in a high vacuum; it is no longer possible to speak of "flow" in the macroscopic sense.

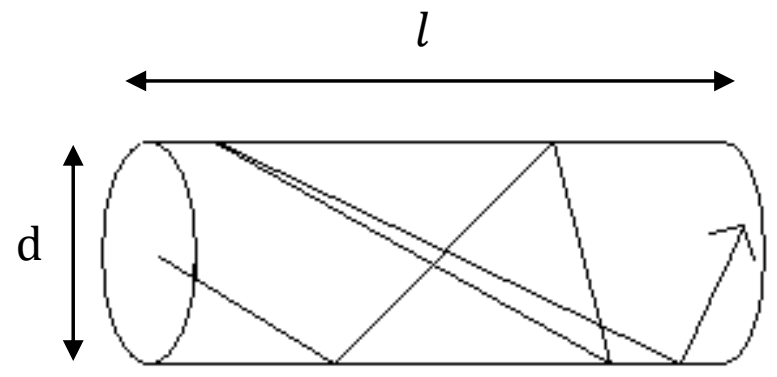

Figure 3.1: Schematic view of the molecular flow in a pipe.

$$
C=\frac{d^{2} \pi}{4} \frac{1}{\left(1+\frac{3}{4} \frac{l}{d}\right)} \sqrt{\frac{R T}{2 \pi M_{m o l}}}
$$

For long tubes with $\mid$ »d:

$$
C_{\text {long }}=\frac{\pi}{3} \frac{d^{3}}{l} \sqrt{\frac{R T}{2 \pi M_{m o l}}}
$$

For short tubes with $1 \approx \mathrm{d}$ :

$$
C_{\text {short }}=\frac{d^{2} \pi}{7} \sqrt{\frac{R T}{2 \pi M_{m o l}}}
$$


For circular aperture:

$$
C=\frac{d^{2} \pi}{4} \sqrt{\frac{R T}{2 \pi M_{m o l}}}
$$

\subsection{Calculation of pump-down time for a vacuum system}

One of the most important considerations by designing a new vacuum system or evaluating the performance of an existing one is to know the effective pumping speed. The effectiveness of a vacuum pump is given in terms of the pump speed $S_{p}$, which is defined like following (Figure 3.2)

$$
S_{p}=Q / p_{i}
$$

where $Q$ is the throughput of the pump and $p_{i}$ is the pressure at the inlet of the pump[46]. The effective pumping speed $S_{s}$ is defined by

$$
S_{e}=Q / p
$$

where $p$ is the pressure within the vacuum space. The overall conductance of the piping system between the vacuum space and the vacuum pump is also related to the throughput by

$$
C_{0}=Q /\left(p-p_{i}\right)
$$

Combining the first and the last equation, we find the following relationship between the pump speed, effective pumping speed, and overall piping conductance.

$$
1 / S_{e}=1 / S_{p}+1 / C_{0}
$$




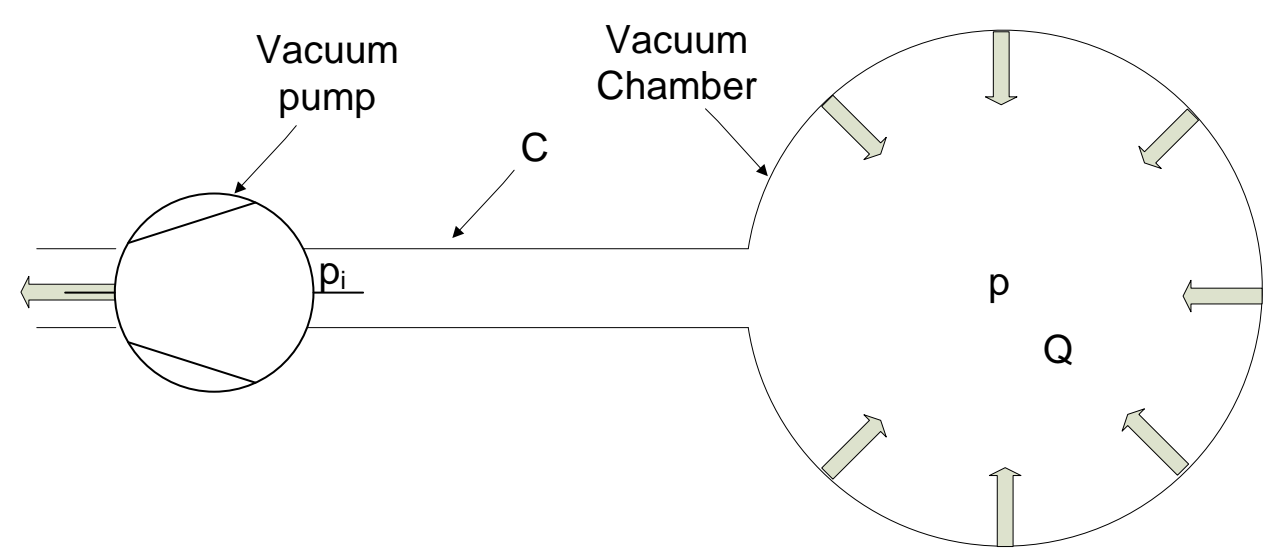

Figure 3.2: Vacuum system for pump- down equation development.

After a long pumping time, the pressure of the system changes only slightly $(d p / d t=0)$ and the system pressure approaches the ultimate pressure of the system $p_{u}$. For constant system pumping speed and constant $Q$, the pump-down time between the initial pressure $p_{1}$ at $t=0$ and the final pressure $p_{2}$ at $t=t_{p}$ is given [46]

$$
t_{p}=\left(\frac{V}{S_{e}}\right) \ln \left(\frac{p_{1}-p_{u}}{p_{2}-p_{u}}\right)
$$

\subsection{Outgassing and bake out}

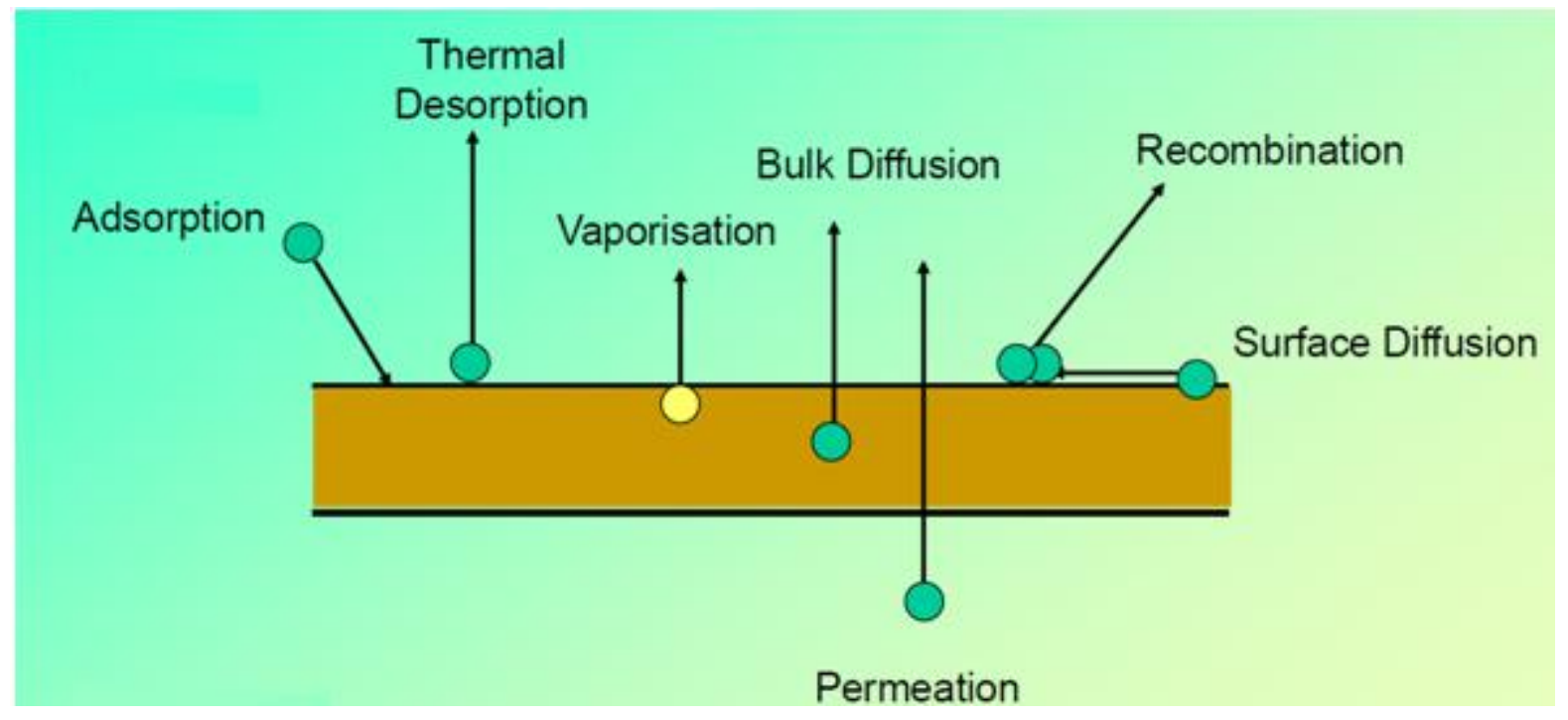

Figure 3.3: Different gas production mechanism in the solid.

Outgassing is the process of releasing of the gas, which is produced through several mechanisms like gas permeation through the walls, vaporisation of materials and thermal 
desorption. It affects the residual pressure in vacuum chamber. At higher temperatures the rate of outgassing increases due to the increase of the vapour pressure, of the rate of chemical reaction and thermal desorption of adsorbed molecules. The outgassing rate depends on the conditions of the surface exposed to the vacuum.

Typical values for the outgassing rate for different materials are shown in Table 3.4.

\begin{tabular}{|c|c|c|c|}
\hline \multicolumn{4}{|c|}{ At room temperature } \\
\hline Standard values & \multirow{2}{*}{\multicolumn{2}{|c|}{$\begin{array}{c}\text { Metals } \\
10^{-9} \ldots 10^{-7}\end{array}$}} & Nonmetals \\
\hline$\left(\operatorname{mbar} \cdot 1 \cdot \mathrm{s}^{-1} \cdot \mathrm{cm}^{-2}\right)$ & & & $10^{-7} \ldots 10^{-5}$ \\
\hline \multicolumn{4}{|c|}{ Outgassing rate } \\
\hline Material & Condition & 1 hour & 10 hour \\
\hline $\mathrm{Al}$ & None & $1.33 \cdot 10^{-6}$ & ---- \\
\hline $\mathrm{Al}$ & Cleaned & ---- & $1.06 \cdot 10^{-8}$ \\
\hline $\mathrm{Al}$ & Degreased & $8.4 \cdot 10^{-9}$ & ---- \\
\hline Stainless Steel & None & $8.5 \cdot 10^{-7}$ & $2.66 \cdot 10^{-8}$ \\
\hline Stainless Steel & Degreased & $5.32 \cdot 10^{-7}$ & ----- \\
\hline Stainless Steel & Baked $24 \mathrm{~h}\left(200^{\circ} \mathrm{C}\right)$ & $1.9 \cdot 10^{-10}$ & ---- \\
\hline
\end{tabular}

Table 3.4: Outgassing rates of different materials (All values depend on pre-treatment) [43].

Outgassing is strongly depending on the treatment of the material. Processes like degreasing, annealing or baking change it. For example the outgassing rate of as received stainless steel after 1 hour pumping time is $8.5 \cdot 10^{-7} \mathrm{mbar} \cdot 1 \cdot \mathrm{s}^{-1} \cdot \mathrm{cm}^{-2}$, of degreased stainless steel after 1 hour pumping time is $5.32 \cdot 10^{-7} \mathrm{mbar} \cdot 1 \cdot \mathrm{s}^{-1} \cdot \mathrm{cm}^{-2}$ and of baked stainless steel again after 1 hour pumping time is $1.9 \cdot 10^{-10} \mathrm{mbar} \cdot 1 \cdot \mathrm{s}^{-1} \cdot \mathrm{cm}^{-2}$.

The residual gas in a vacuum system resides mostly on the walls, however at room temperature the molecules occasionally desorb and fly across the system. The very weakly physisorbed molecules are not a problem, since they will come off in a relatively short time and are pumped away. Nor the very strongly chemisorbed ones are a problem, since they will desorb too rarely to influence the residual pressure. The molecules in the intermediate range are the biggest concern, which means adsorbed molecules with binding energies of 70 to $100 \mathrm{kj} / \mathrm{mol}$. The reason is the very long sojourn time, mostly of the order of one year at room temperature. The only practical way to remove these molecules is to bake the system. 


\subsection{Adsorption}

Adsorption is a process by which the gas molecules or atoms stick to the surface of the solid in one or more layers. The adsorption-desorption process is controlled by the interaction energy between the adsorbed molecules or atoms and the surface atoms. This surface process is easily mistaken for absorption, which is the penetration of the gas molecules into the solid.

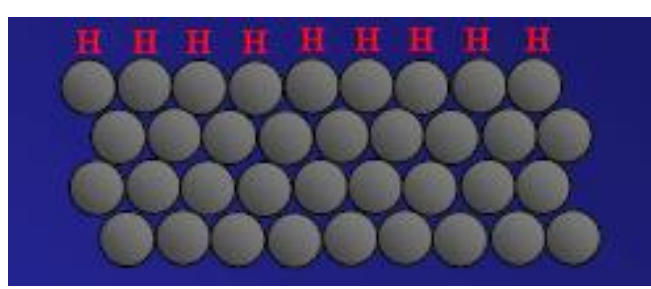

$\mathrm{H}_{2}$ adsorption

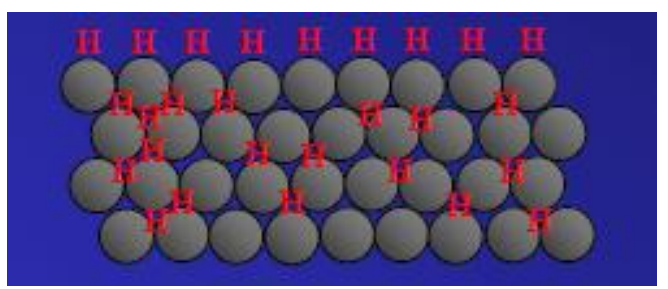

$\mathrm{H}_{2}$ absorption

Figure 3.4: Schematic description of adsorption and absorption.

According to the binding energy adsorption can be divided in two groups:

Weak: physical adsorption (Physisorption) $\mathrm{E}<30 \mathrm{kj} / \mathrm{mol}$

Strong: chemical adsorption (Chemisorption) E> $100 \mathrm{kj} / \mathrm{mol}$

Physisorption is based on the Van der Waals interactions between the adsorbate and the substrate (and also between the adsorbate molecules). Almost all gases can physisorb under the correct conditions as for instance low temperature to almost all surfaces. Since there is no covalent bond formation, there is no electron exchange between the adsorbed molecule and substrate surface. The geometrical structure and electronic characteristics of the adsorbed molecule or atom and also of the surface are essentially preserved. A physisorbed molecule can spontaneously leave the surface after a certain time. The energy necessary in order to desorb a physisorbed molecule has the same order of magnitude as the condensation enthalpy. Physisorption is therefore observed mostly at low temperatures.

Chemisorption occurs when there is the formation of a chemical linkage between adsorbate and substrate. In this case, the enthalpy is around an order of magnitude higher than with physisorption.

Both physical and chemical adsorption may occur on a substrate at the same time; a layer of molecules may be physically adsorbed on top of an underlying chemisorbed layer. It is also possible for a gas to be physically adsorbed at first, and then to react chemically more slowly with the surface of the solid. At low temperatures, chemisorptions may be so slow that only 
physisorption occurs. Therefore in cryogenic temperatures the important adsorption is physisorption.

The interaction between two uncharged molecules is described by Lennard-Jones potential (Figure 3.5). It is mildly attractive as two uncharged molecules or atoms approach one another from a distance, but strongly repulsive when they approach too close.

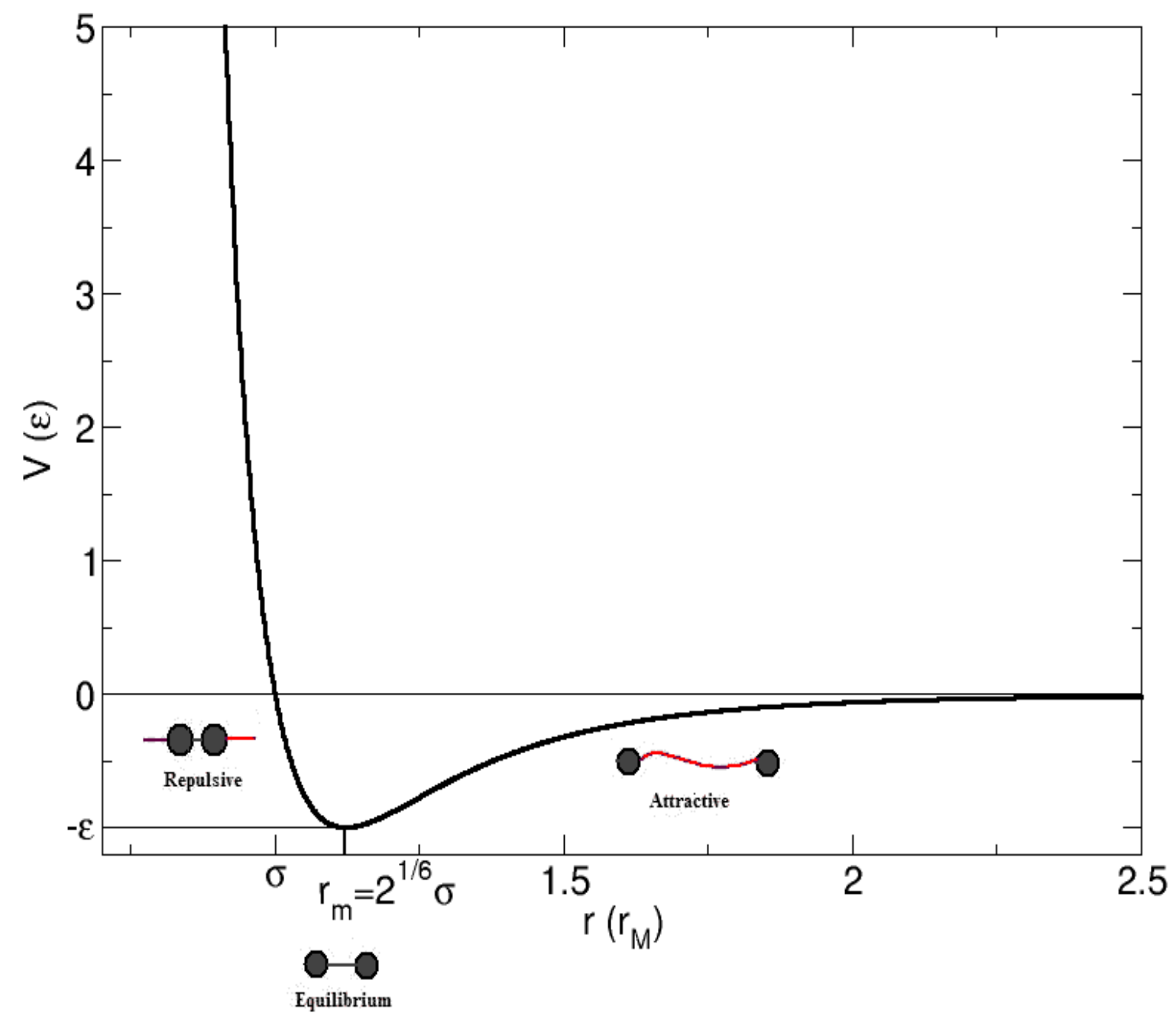

Figure 3.5: Lennard-Jones potential.

Lennard-Jones potential is given by:

$$
E=\varepsilon\left[\left(\frac{r_{m}}{r}\right)^{12}-2\left(\frac{r_{m}}{r}\right)^{6}\right]
$$

$\varepsilon$...Depth of potential well

$r_{m}$...Equilibrium distance from the surface

r...Distance from the surface 
The BET (Stephen Brunauer, Paul Hugh Emmett and Edward Teller) equation represents the physical adsorption data for gas temperatures below the critical temperature of the gas. When a gas molecule strikes the bare surface of the adsorbent, the molecule is retained on the surface if its energy is lower than the interaction energy between the molecules and the adsorbent atoms. The adsorbent is covered with a layer of gas molecules one molecule thick (monolayer). Molecules continue to strike the surface that is covered with adsorbed gas molecules to form a layer of molecules two molecules thick. It continues like that till the equilibrium is reached.

The BET equation is like following [46]:

$$
\frac{V}{m_{a}}=\frac{v_{m} z\left(p / p_{\text {sat }}\right)}{\left(1-p / p_{\text {sat }}\right)\left[1+(z-1)\left(p / p_{\text {sat }}\right)\right]}
$$

where $\quad$ V... volume of gas adsorbed at 1 bar and $273.2 \mathrm{~K}$

$\mathrm{m}_{\mathrm{a} . . .}$ mass of adsorbent

$\mathrm{v}_{\mathrm{m}} \ldots$ volume of gas per unit mass of adsorbent required to form a monolayer over the entire adsorbent surface

p...partial pressure of the gas being adsorbed

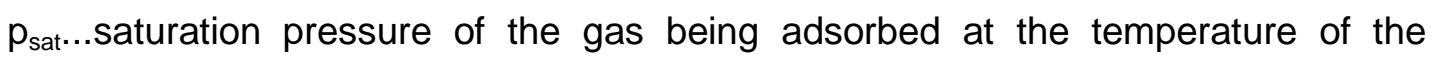
adsorbent

The parameter $z$ is a function of the energy of adsorption and the temperature of the adsorbent,

$$
z=\exp \left(\theta_{a}[K] / T[K]\right)
$$

The sticking coefficient is defined as the ratio of the number of molecules that are captured and stick to the surface to the number of molecules that strike the surface. The warm surface is assumed to have a sticking coefficient of zero. The sticking factor may change for different molecules but for SEY measurements we assumed that the sticking factor at $4,7 \mathrm{~K}$ is 1 for each gas. The first monolayer that adsorb will have the highest sticking coefficient. As further monolayers form, the sticking coefficient becomes even lower as the nature of the surface changes and the subsequent bonds become weaker.

If the adsorbed molecule possesses sufficient energy to emerge from the potential well in which it is trapped, desorption occurs. Desorption can be activated by thermal energy (Thermal desorption) or by energy transfer from photons, electrons, ions etc (Stimulated 
desorption). Another way of desorption is called reaction before desorption, where adsorbed atoms form molecules and then the molecules leave the surface. 


\section{Design of the system for SEY- Measurements at cryogenic temperatures}

\subsection{Vacuum Chamber}

In order to measure the secondary electron yield of physisorbed gases at cryogenic temperatures a new vacuum system has been designed. The measurement assembly is mounted in a bake-able, all metal UHV system. The system can be divided in 3 main systems. The first part shall be called the cryogenic system. It consists of a cold head and the sample connected to the cold head. It is used to cool down the sample with liquid helium and to keep the sample at a temperature of $4,7 \mathrm{~K}$. Because of the heat radiation coming from the warm vacuum chamber, it is not possible to cool down to $4,2 \mathrm{~K}$, which is in fact the cryogenic liquid helium temperature. The second part shall be called the vacuum system. The pressure in the vacuum system is in the range of $9 \cdot 10^{-10} \mathrm{mbar}$. The cryogenic system and the vacuum system are connected via a bellow, which allows us to adjust the distance between the sample and the collector as well between the sample and the Kelvin Probe. The vacuum system is equipped with the following instrumentation:

- a 60l/s turbo molecular pump

- Residual Gas Analyzer (RGA) and a Bayard Alpert Gauge, which are used for vacuum diagnostics and for the quantitative evaluation of adsorbates on the sample. With the help of the RGA, which is based on a mass spectrometry, it is possible to detect leaks and outgassing. This device can do the same work as a helium leak detector and much more. 
- a Penning gauge to measure the pressure in the main chamber

- an electron gun with a spot size/beam current of $1 \mathrm{~mm} / 2 \cdot 10^{-7} \mathrm{~A}$ and beam energy of $50 \mathrm{eV}-2500 \mathrm{eV}$

- a Kelvin Probe to perform the work function measurements

The third part is the gas injection system, which will be used to inject and adsorb different gases (CO, $\mathrm{CO}_{2}, \mathrm{H}_{2}, \mathrm{CH}_{4}, \mathrm{C}_{2} \mathrm{H}_{6}, \mathrm{Kr}$ ) for measuring $\mathrm{SEY}$ of physisorbed gases and water injection line to physisorb water.

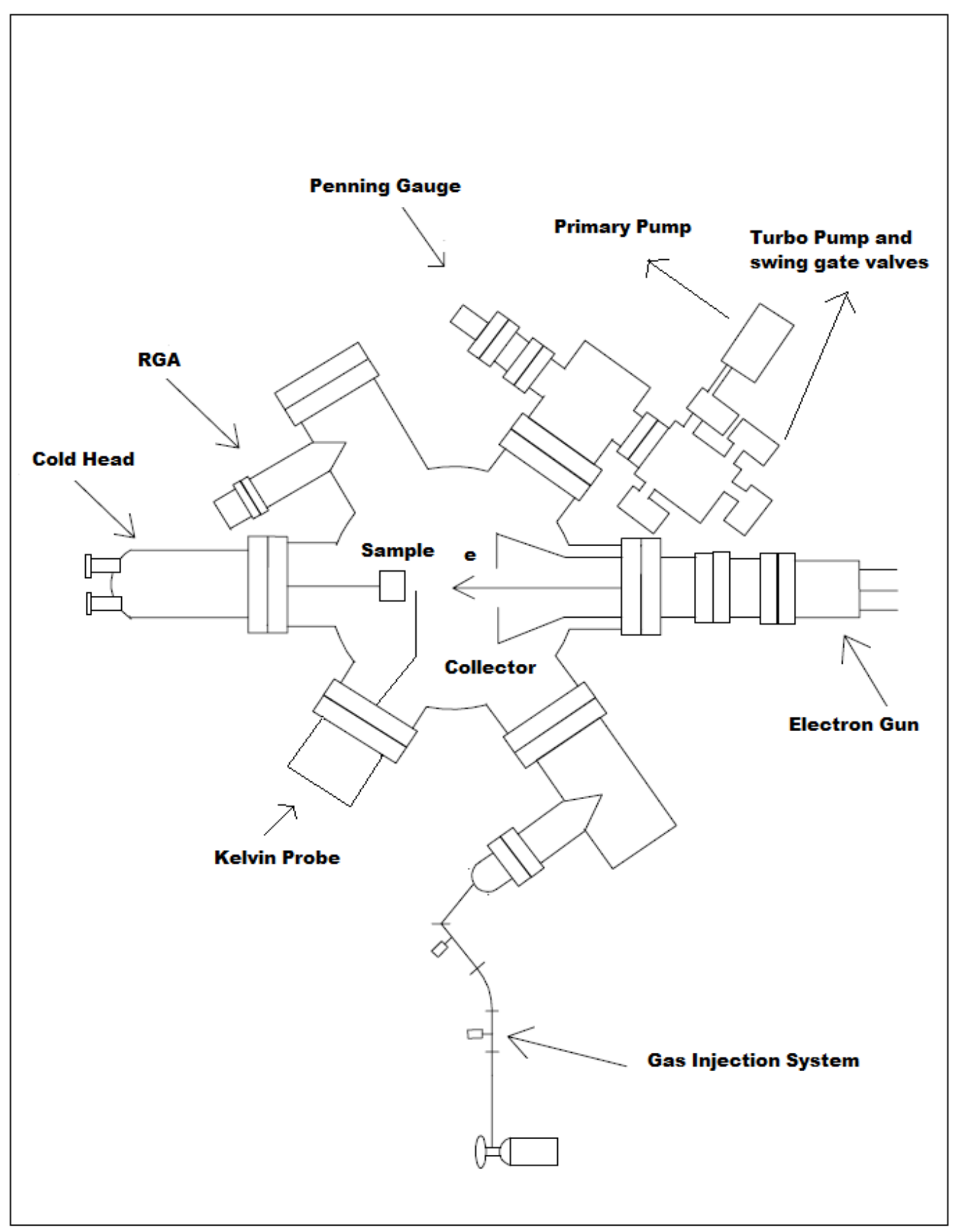

Figure 4.1: Schematic view of the measurement system for cryogenic measurements. 
Chapter 4- Design of the system for SEY-Measurements at cryogenic temperatures

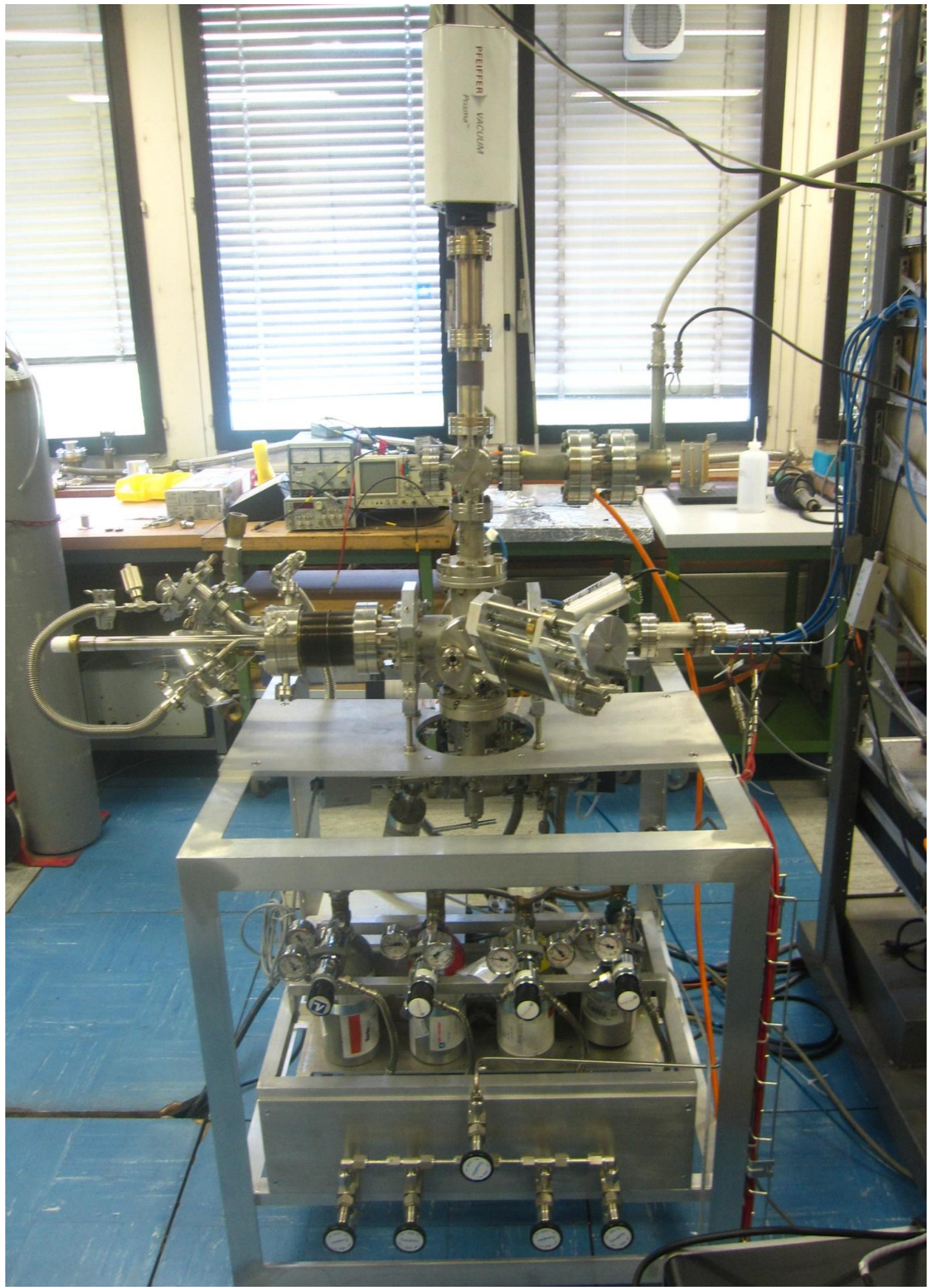

Figure 4.2: System for SEY measurements. 


\section{Chapter 4- Design of the system for SEY-Measurements at cryogenic temperatures}

The whole system is made of stainless steel $316 \mathrm{LN}$, where the pressure is in the high $10^{-8}$ mbar without bake-out. The flanges are Conflat $^{\mathrm{TM}}$ flanges with different nominal diameters like 16,40 and $63 \mathrm{~mm}$.

All metal valves with O-ring seals have been used. Also a leak valve with a sapphire crystal as a sealing surface area has been assembled on the gas injection system for controlled inlet of gases into the UHV chamber. A rupture disk is mounted on the system to protect it from overpressure in case of a leak from the gas bottles into the system ( the gas bottles are under 200bar pressure).

To assemble the flanges, windows and all other instruments silver plated screws were used, since uncoated screws may seize after baking. Also silver plated $\mathrm{Cu}$-seals were used to join the flanges. For the connections of the outlets and the inlets of the cold head Helicoflex ${ }^{\mathrm{TM}}$ seals have been used.

To have an ultrahigh vacuum system for SEY measurements, bake out has been performed each time, after the system had been opened. The pressure of the non baked system is about $6 \times 10^{-8} \mathrm{mbar}$. After bake pressures of $9 \times 10^{-10} \mathrm{mbar}$ could be reached. So the pressure was almost 2 magnitudes lower than the non baked system. Before bake-out the dominant gas in the system is water, where else after bake out the amount of water decreases and the dominant gas is $\mathrm{H}_{2}$ in the spectrum.

To which temperature and for how long a system has to be heated depends on the desired ultimate pressure, on the binding energies of the molecules, the material of the vessel and the sealings and also on the surface of the vessel and its pre-treatment. Different elements of the system like RGA, B\&A gauge, electron gun are allowed to be heated up to different maximal temperatures, depending on the sensitivity of the materials used to build them. The bake out procedure used in SEY system had 4 different programs each with 3 steps. The programs and the steps used for each element are shown in the table 3.5 and 3.6. 
Chapter 4- Design of the system for SEY-Measurements at cryogenic temperatures

\begin{tabular}{|c|c|}
\hline Element & Programme \\
\hline Cold Head & 1 \\
\hline Residual Gas Analyzer & 2 \\
\hline Bayard \& Alpert Gauge & 1 \\
\hline Electron gun & 1 \\
\hline Main chamber & 4 \\
\hline Angle valve & 1 \\
\hline Kelvin Probe & \\
\hline
\end{tabular}

Table 3.5: Number of the programme used for the bake out of different elements.

\begin{tabular}{|c|ccc|ccc|ccc|}
\hline Prog & ${ }^{\circ} \mathbf{C} / \mathbf{h}$ & $\mathbf{T}_{\max }$ & $\mathbf{t}(\mathbf{h})$ & ${ }^{\circ} \mathbf{C} / \mathbf{h}$ & $\mathbf{T}_{\max }$ & $\mathbf{t}(\mathbf{h})$ & ${ }^{\circ} \mathbf{C} / \mathbf{h}$ & $\mathbf{T}_{\max }$ & $\mathbf{t}(\mathbf{h})$ \\
\hline 1 & 100 & 150 & 20 & 100 & 100 & 40 & 100 & 20 & 48 \\
2 & 200 & 250 & 20 & 200 & 100 & 40 & 100 & 20 & 48 \\
3 & 50 & 120 & 20 & 50 & 100 & 40 & 100 & 20 & 48 \\
4 & 50 & 140 & 20 & 50 & 100 & 40 & 100 & 20 & 48 \\
\hline
\end{tabular}

Table 3.6: Maximum temperature and the time for each temperature for different programmes.

\subsection{Gas injection system}

The residual gas in a UHV system like in LHC can affect the SEY value. In cryogenic temperatures between $5 \mathrm{~K}$ and $20 \mathrm{~K}$ the residual gas condenses on the surface of the chamber wall and changes the SEY. To investigate the changes of SEY as a function of physisorbed gases a gas injection system has been designed and built. The main focus was on the following gases: $\mathrm{N}_{2}, \mathrm{CO}, \mathrm{CO}_{2}$, and $\mathrm{CH}_{4}$. Also measurements with $\mathrm{Kr}$ and $\mathrm{C}_{2} \mathrm{H}_{6}$ have been performed. The expected gas composition on the beam screen during operation is $\mathrm{H}_{2}$, $\mathrm{CH}_{4}, \mathrm{CO}$ and $\mathrm{CO}_{2}$, that's the reason why the measurements with $\mathrm{CH}_{4}, \mathrm{CO}$ and $\mathrm{CO}_{2}$ have been performed. $\mathrm{H}_{2}$ is not measured because at a pressure of $10^{-8} \mathrm{mbar}, \mathrm{H}_{2}$ starts to evaporate over a temperature of $3.7 \mathrm{~K}$ and we could cool down the sample to $4.7 \mathrm{~K}$. With the system used for SEY measurements it was possible to go down till 4.7K but not more, so that we couldn't perform measurements with $\mathrm{H}_{2}$. SEY of $\mathrm{N}_{2}$ has been measured, because this is the gas used in all gauges and pumps as the calibration gas. Measurements with $\mathrm{Kr}$ 


\section{Chapter 4- Design of the system for SEY-Measurements at cryogenic temperatures}

have been performed because there was the chance to compare the results with literature values.

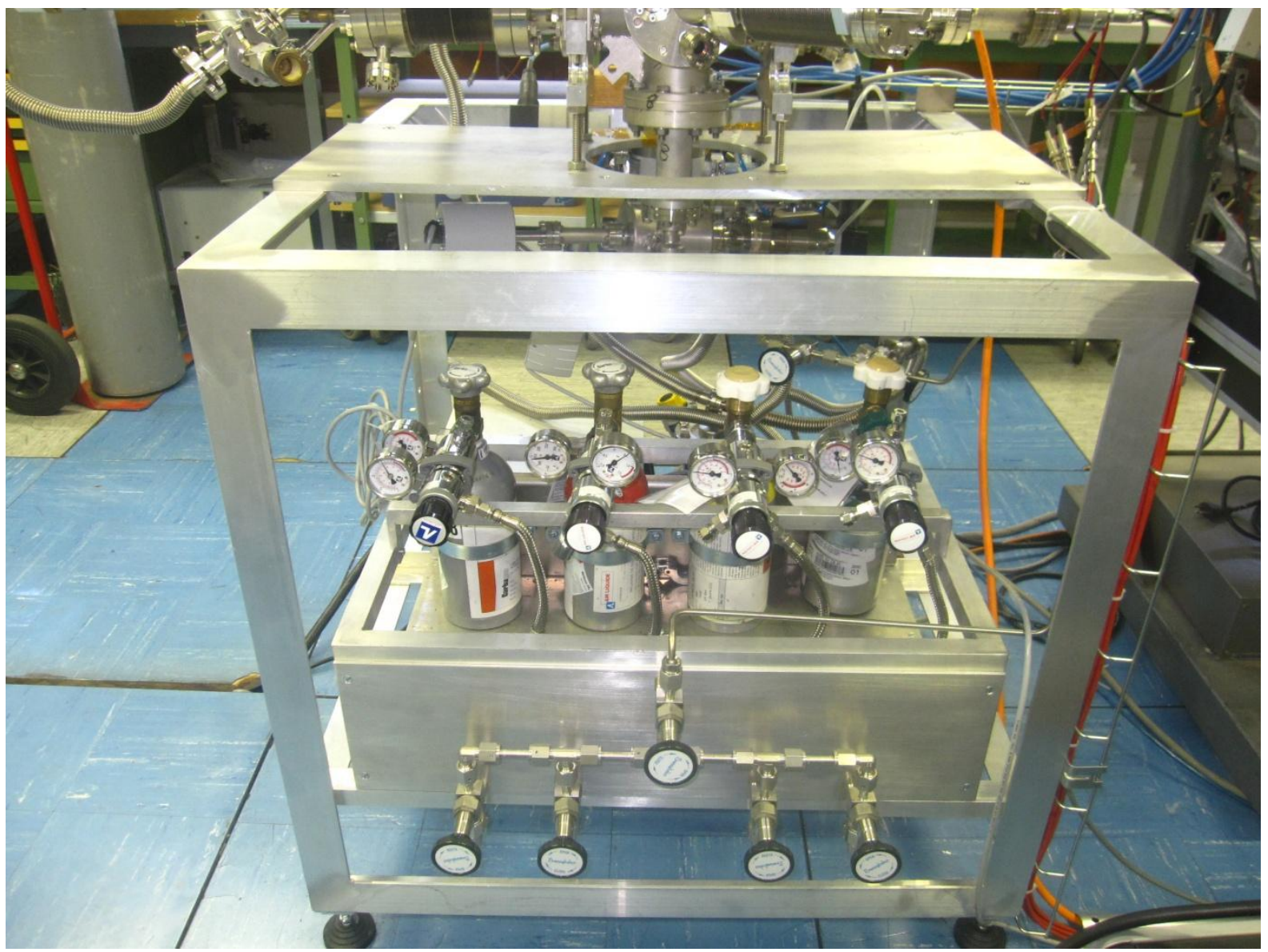

Figure 4.3: Gas injection system.

The gas injection system consists of 4 gas cylinders, each one equipped with a pressure reducing valve. The gas flows to a volume of $1 / 2$ litres through a variable leak valve, and the volume is separated from the main chamber with an all metal valve, where also a known conductance is installed in order to be able to calculate the gas flow. A primary pump and a turbo pump with a pumping speed of $60 \mathrm{l} / \mathrm{s}$ are attached to the system to pump down the injection line after and before the measurements. One compact capacitance gauge CMR 274 and one Compact Process lon gauge IMR 265 are used to measure the pressure in the half litre volume.

- Compact capacitance gauge CMR 274:

It is made of an aluminium-oxide-ceramic capacitance and the electronic system converts the capacitance in an output voltage. (Pressure range from $1.1 \mathrm{mbar}$ to $10^{-3} \mathrm{mbar}$ ) 


\section{Chapter 4- Design of the system for SEY-Measurements at cryogenic temperatures}

- Compact process ion gauge IMR 265:

It is made of a Pirani gauge and a hot-cathode gauge. The pressure range is from $10^{3} \mathrm{mbar}$ to $2 \cdot 10^{-6}$ mbar. From $10^{3}$ mbar to $5 \times 10^{-2}$ mbar the measurement is done by the Pirani gauge and by lower pressures hot-cathode gauge starts to work.

To build the gas injection system VCR fittings of Swagelok were used, because of their high cleanliness and because they are suitable in low pressure measurements. The seal on a VCR assembly is made when the gasket is compressed by two beads during the engagement of a male nut or body hex and a female nut. Stainless steel tubes with an inner diameter of $6 \mathrm{~mm}$ were used for the injection line. In total 7 Swagelok SS-4BG-V51 stainless steel valves were used, which are compatible for working pressures up to 68,9 bar.

A volume of $1 / 2$ litres was manufactured and mounted to have a well-known amount of gas, which will then flow to the main chamber. One variable leak valve (Varian 951-5106) was attached to the volume to have a controlled gas flow to the defined volume.

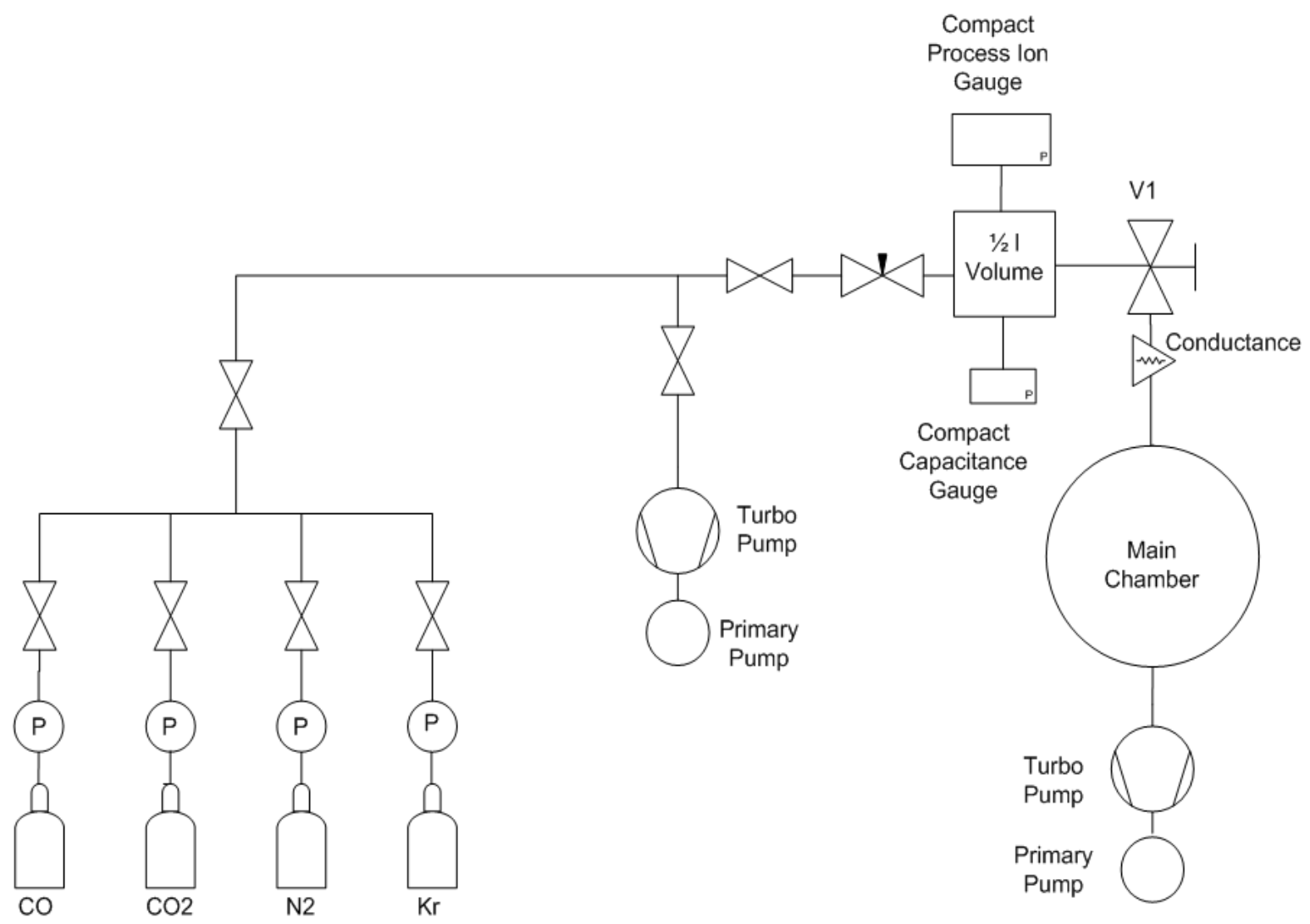

Figure 4.4: Drawing of gas injection system, which has been used for gas adsorption in cryogenic temperatures. 

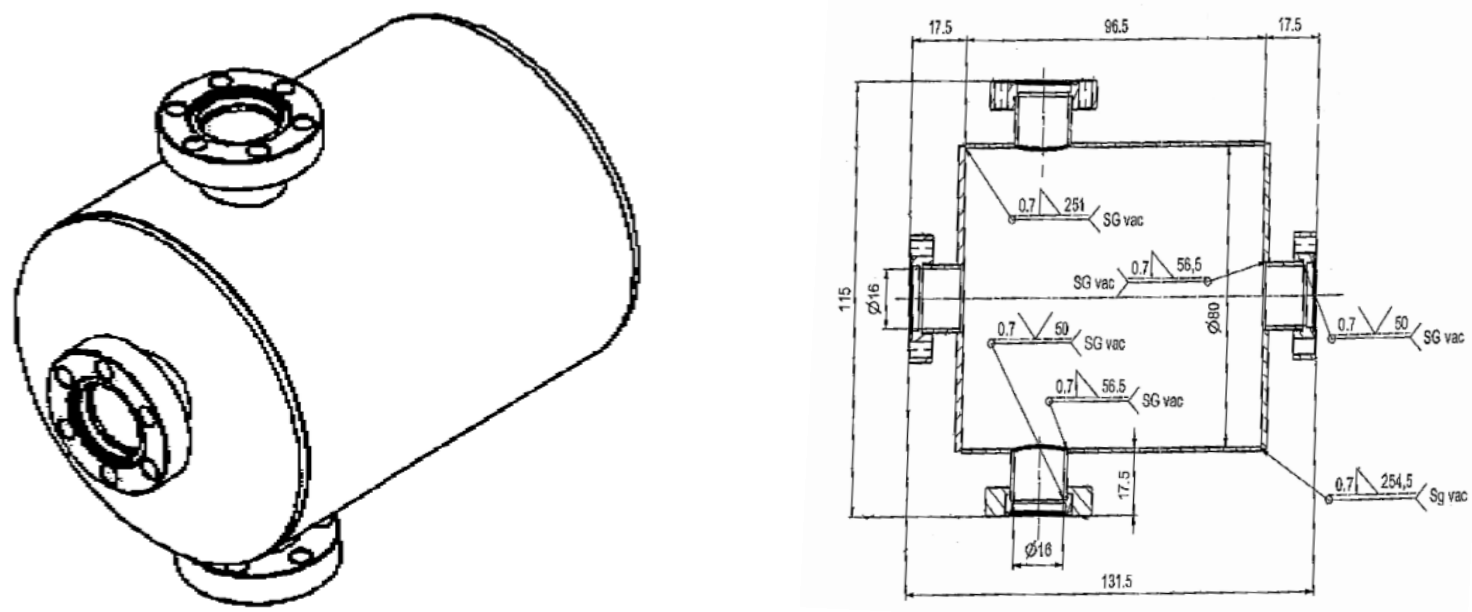

Figure 4.5: 1/2 litre volume, to let a well-known amount of gas flow in the main chamber.

\subsection{Calibration of gas injection system}

Once the sample is cooled down to the cryogenic temperatures $(4.7 \mathrm{~K})$ it is possible to adsorb different gases and measure SEY as a function of the physisorbed gas.

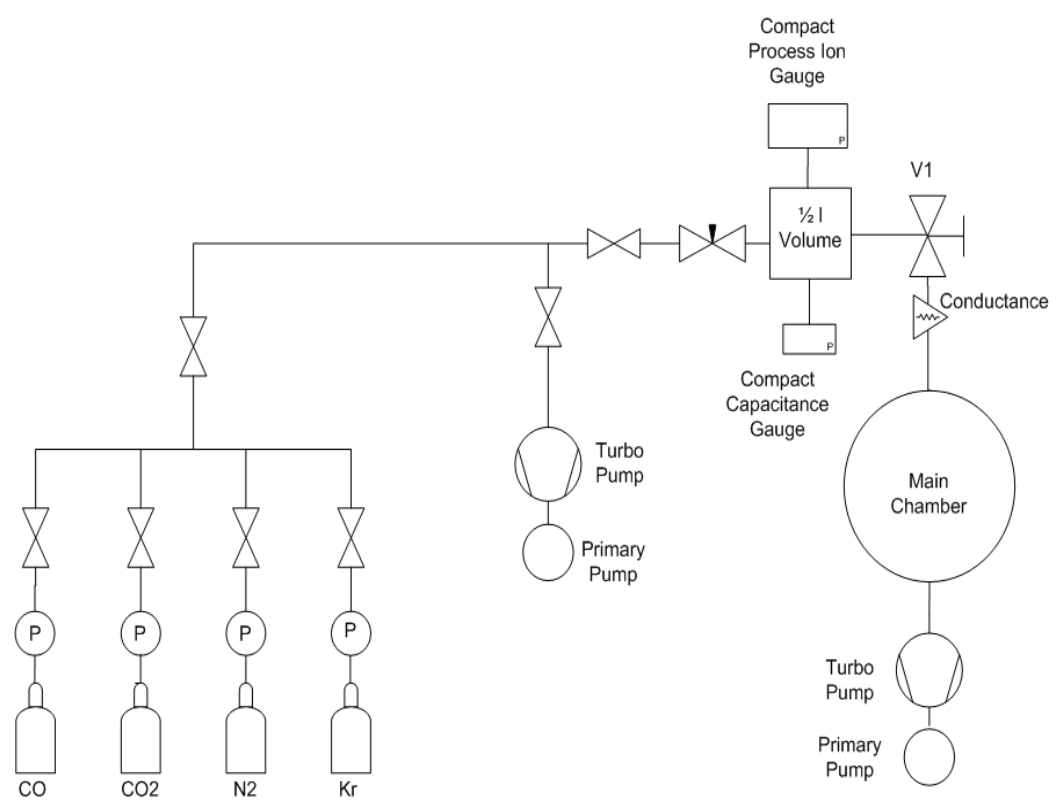

First the volume will be filled with gas of known pressure. Later, the gas will be let through a conductance in the main chamber by opening the valve $\mathrm{V} 1$. In order to know the gas flow to the main chamber and accordingly the number of monolayers adsorbed on the sample the following calculations have been done. 


\section{Chapter 4- Design of the system for SEY-Measurements at cryogenic temperatures}

\subsubsection{Measuring the gas flow into the well defined volume}

For manufacturing the conductance, a nominal diameter $d$ of $0.5 \mathrm{~mm}$ was selected. According to the formula of conductance for monocular flow (see chapter 3.3), the conductance for the $\mathrm{N}_{2}$ was calculated.

In order to verify the conductance, the gas flow into the well defined volume had to be measured. Gas with a constant pressure from the bottle and accordingly constant flow has been let into the volume, while the valve V1 has been kept closed. The pressure increase in the volume has been measured for a defined time of gas inlet. The gas flow can be calculated like following:

$$
Q_{i n}=\frac{\Delta p \cdot V}{\Delta t}
$$

where $\Delta p$ is the pressure change in the volume and $\Delta t$ is the time. After opening the valve $\mathrm{V} 1$, the flow remains constant and steady state will be reached after some time. The conductance can be calculated by

$$
C=\frac{Q_{\text {in }}}{p_{2}}
$$

where $p_{2}$ is the pressure in the main chamber $(V=3.2$ litre).

The calculated and the measured values of the conductance for $\mathrm{N}_{2}$ match. The conductance for other gases could be calculated by using the formula below:

$$
\frac{C_{x}}{C_{y}}=\sqrt{\frac{M_{y}}{M_{x}}}
$$

The calculated and measured valued for conductance are shown in table 3.6. The values for conductance in the second column are values calculated by using the formula above and the value on the third column for $\mathrm{N}_{2}$ shows the measured value. 
Chapter 4- Design of the system for SEY-Measurements at cryogenic temperatures

\begin{tabular}{|c|c|c|}
\hline Gas & C (I/s) calculated & C (I/s) measured \\
\hline $\mathrm{CH}_{4}$ & 0.036 & \\
\hline $\mathrm{H}_{2} \mathrm{O}$ & 0.034 & \\
\hline $\mathrm{N}_{2}$ & 0.027 & 0.027 \\
\hline $\mathrm{CO}$ & 0.027 & \\
\hline $\mathrm{C}_{2} \mathrm{H}_{6}$ & 0.026 & \\
\hline $\mathrm{CO}_{2}$ & 0.022 & \\
\hline $\mathrm{Kr}$ & 0.016 & \\
\hline
\end{tabular}

Table 3.6: Calculated and measured conductance of different gases used for the gas adsorption.

\subsubsection{Pumping speed of the pump and the cold sample}

To calculate the number of the monolayers adsorbed on the cold sample, it is necessary to know the exact pumping speed of the cold sample. For that purpose first the pumping speed of the pump at room temperature has been measured for each gas by using the injection line and the known conductance. For room temperature the pumping speed of the sample for each gas is negligible. Afterwards the sample has been cooled down to measure the pumping speed of it.

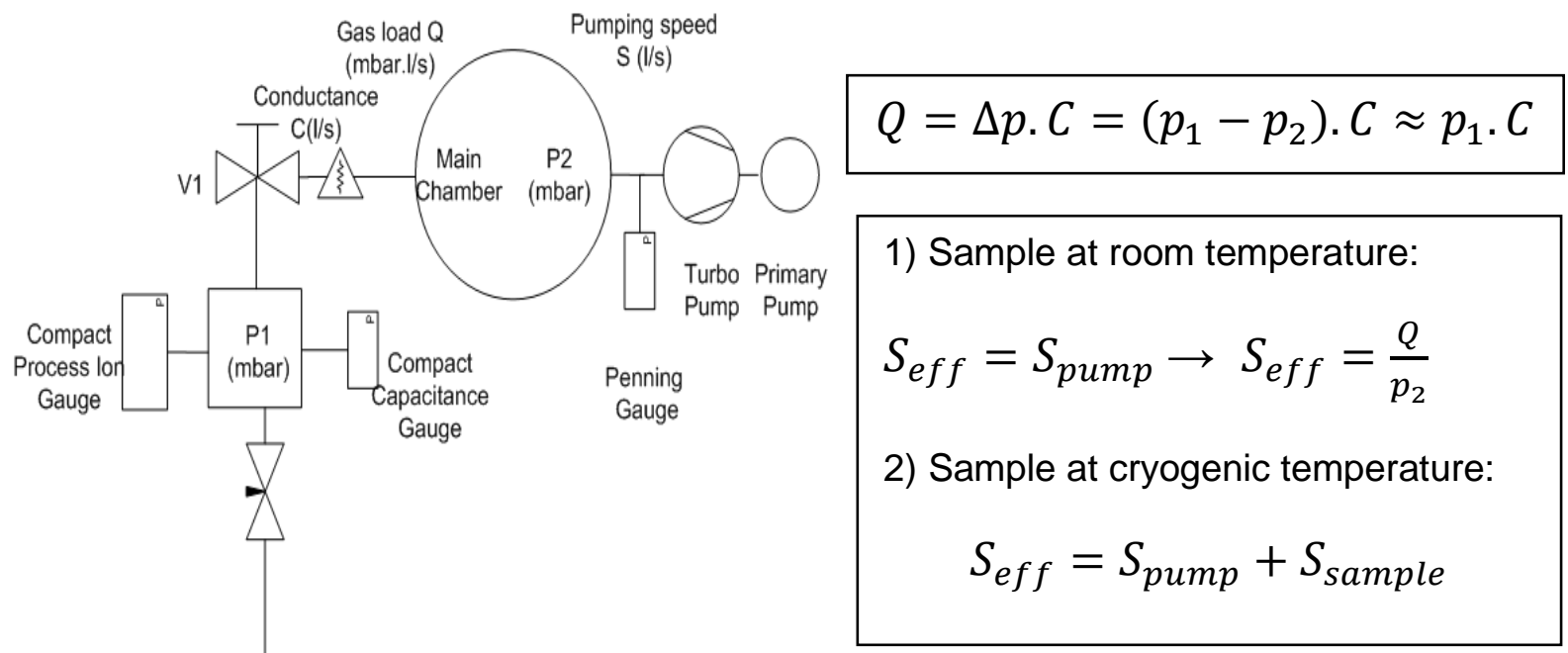


Chapter 4- Design of the system for SEY-Measurements at cryogenic temperatures

\begin{tabular}{|c|c|c|c|c|}
\hline Gas & $\mathrm{S}_{\mathrm{Cu}}(\mathrm{I} / \mathbf{s})$ & $\mathrm{S}_{\text {AI }}(\mathrm{I} / \mathbf{s})$ & $\mathrm{S}_{\mathrm{Cu}, \text { pol }}(\mathrm{I} / \mathbf{s})$ & $\mathbf{S}_{\text {pump }}(\mathrm{I} / \mathbf{s})$ \\
\hline $\mathrm{CH}_{4}$ & 23 & 20 & 23 & 36 \\
\hline $\mathrm{H}_{2} \mathrm{O}$ & 21 & 22 & 21 & 31 \\
\hline $\mathrm{N}_{2}$ & 17 & 18 & 17 & 25 \\
\hline $\mathrm{CO}$ & 17 & 18 & 17 & 25 \\
\hline $\mathrm{CH}_{4}$ & 23 & 20 & 23 & 36 \\
\hline $\mathrm{CO}_{2}$ & 14 & 16 & 14 & 17 \\
\hline $\mathrm{Kr}$ & 10 & 10 & 10 & \\
\hline
\end{tabular}

Table 3.7: Measured pumping speed of the different samples for different gases and the pumping speed of the pump for each gas.

\subsubsection{Calculation of the coverage}

After knowing the pumping speed of the sample, the number of the monolayers adsorbed on the cold sample can be calculated.

$R=83.14$ mbar.l/(mol. $K) \quad V=S_{\text {sample }} . t$

$p \cdot V=n \cdot R \cdot T \rightarrow n=\frac{p . V}{R \cdot T}, \quad$ number of mols of adsorbed gas on the sample

$N_{\text {molecules }}=n \cdot N_{A}, \quad$ number of molecules of adsorbed gas on the sample

$N_{M L}=3 \cdot \frac{10^{15}}{\mathrm{~cm}^{2}} \quad, \quad$ number of molecules in $1 \mathrm{ML}$ of gas $/ \mathrm{cm}^{2}$ (considered identical

for each gas)

$\chi_{M L}=\frac{N_{\text {molecules }}}{N_{M L}}, \quad$ number of monolayers adsorbed in a defined time 


\subsection{Water injection line}

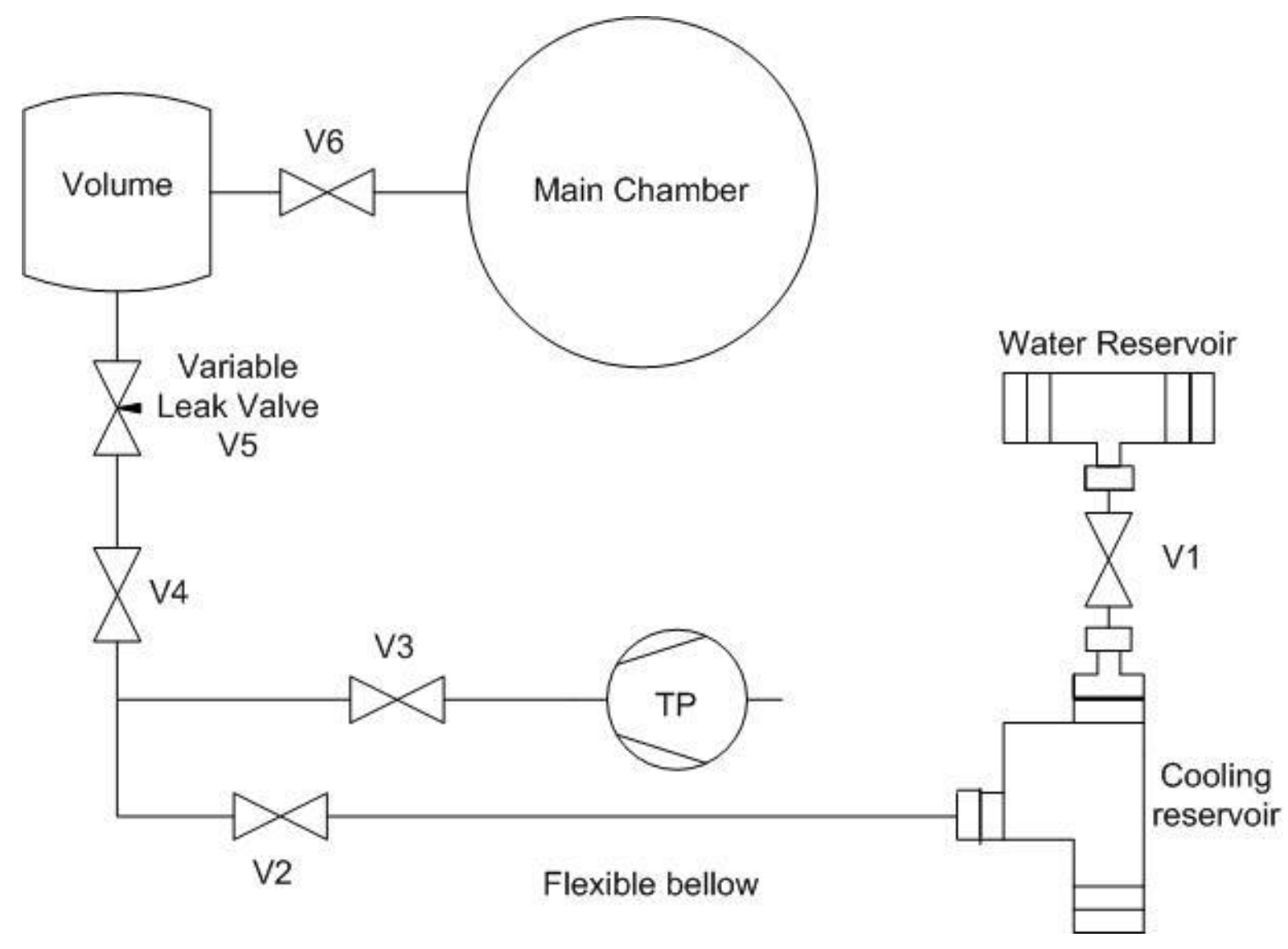

Figure 4.6: Design of the water injection line.

A water injection line has been designed and built to measure the effect on the SEY of physisorbed water on an electro polished copper substrate as a function of the dose of electrons (Figure 4.6 and 4.7). While cooling down the cooling reservoir the valves V1, V4, V5 and V6 are closed and the valves V2 and V3 are open. So the cooling reservoir gets pumped down by the turbo pump. After reaching $-15^{\circ} \mathrm{C}$ on the cooling reservoir via liquid nitrogen, the valve $\mathrm{V} 1$ will be opened and the water vapour reaches the cold part and gets condensed on the wall. After approximately 5 minutes the valves $\mathrm{V} 1$, V2 and $\mathrm{V} 3$ will be closed and the cooling reservoir will be warmed up again. Like that we get app. $20 \mathrm{mbar}$ of $\mathrm{H} 2 \mathrm{O}$ in the reservoir. By opening the valves V4 and V5 carefully, we can have a controlled gas flow to the volume and therefore to the main chamber and perform the adsorption of water on the cold substrate. The measurement and the results will be discussed in the chapter 5 more in details. 


\section{Chapter 4- Design of the system for SEY-Measurements at}

cryogenic temperatures

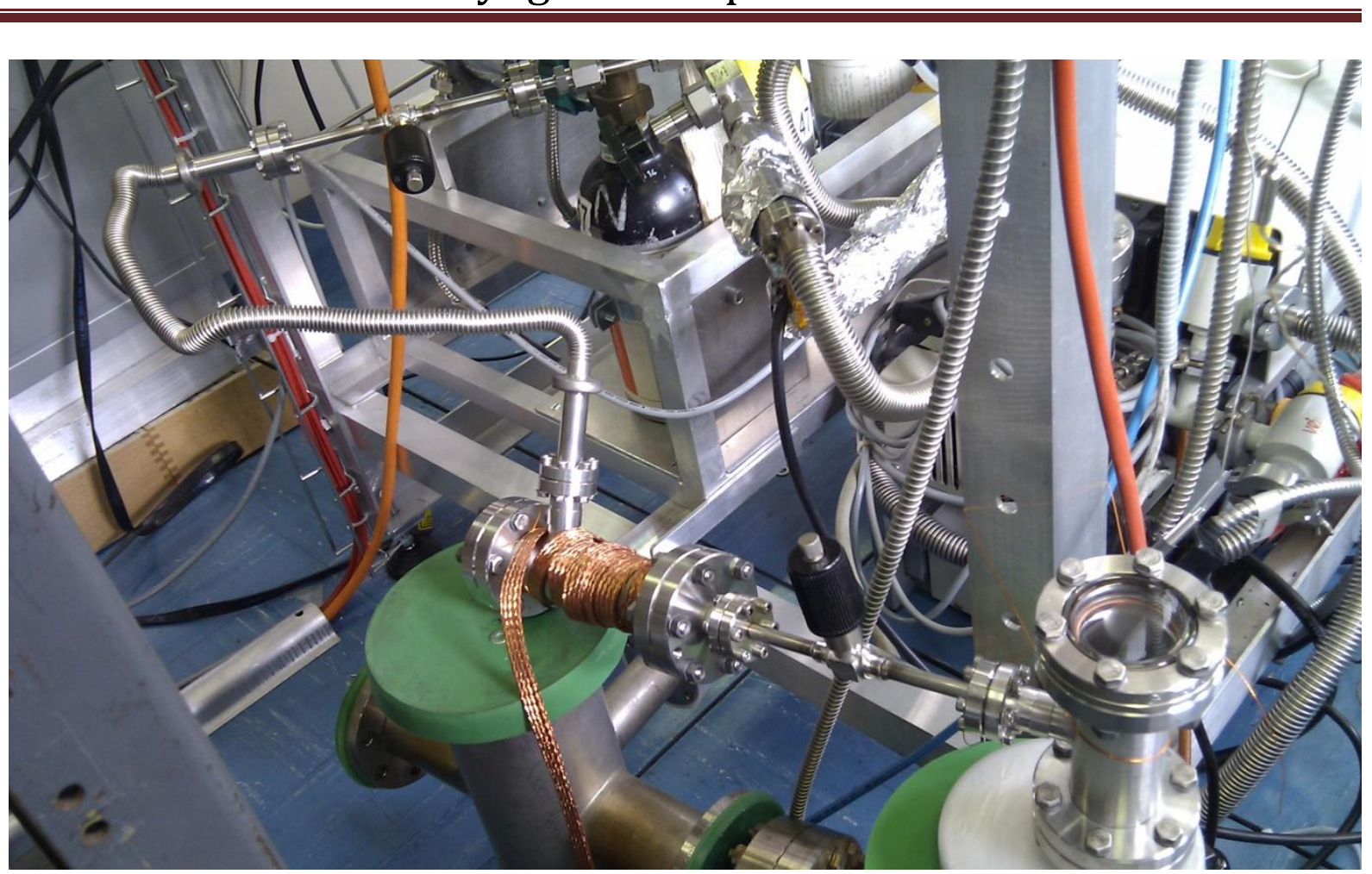

Figure 4.7: Photo of the water injection line.

\subsection{SEY measuring system}

For SEY measurements the target has been irradiated with pulsed primary electrons with a variable energy between $70 \mathrm{eV}$ and $1200 \mathrm{eV}$. Pulsing of the beam could be done by deflecting the beam with a voltage. In order to produce the primary electrons an electron gun (model "ELS5000" from the manufacturer PSP Vacuum Technology) was used. The gun consists of a source mounted on a CF35 flange and an electronic control unit to operate the filament and control the beam energy, intensity, focusing and steering of the beam. Electrical connections between the gun and the control unit are made via the 12-way CF35 feedthrough flange with a lockable 12-way plug and cable assembly. The electron source is bakeable to $150^{\circ} \mathrm{C}$.

The typical parameters of the beam used for the measurements are like following:

- spot size $=1 \mathrm{~mm}$

- $\quad$ pulse time $=2 \mathrm{~ms}$

- beam current $=1.10^{-7} \mathrm{~A}$

- total dose $/$ point $=6 \mathrm{nC} / \mathrm{mm}^{2}$ 


\section{Chapter 4- Design of the system for SEY-Measurements at cryogenic temperatures}

The gun consists of 6 main elements (See Figure 4.8).

1. W hairpin filament mounted in a grid assembly (Wehnelt).

2. Anode lens element.

3. Focus lens element.

4. Ground lens element.

5. Quadrupole beam steering deflectors.

6. End of gun element/nose cone.

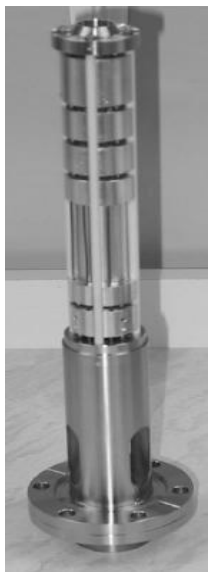

Anode and Earth element are directly connected to ground. The end of gun element is directly attached to ground to maintain a field free region between sample and gun. The gun has been fitted with mu-metal sleeve (thickness of $0.4 \mathrm{~mm}$ ) to act as a magnetic screen. The sleeve is designed to stop potentials on gun elements and gun ceramics affecting the sample potential. The mu-metal provides a magnetic screen which is particularly useful for low beam energies (below $500 \mathrm{eV}$ ). The electrons, emitted by the hot cathode, pass through a lens system and deflector plates to produce a focused beam. The focused beam arrives on the sample and produces secondary electrons. Secondary electrons leaving the sample get collected on the collector; which is situated in front of the sample. The collector has a conical shape in order to be able to collect the secondary electrons in whole $2 \pi$ azimuthal range. The conical shape of the collector doesn't allow the secondaries to escape the collector. The collector is biased at $+45 \mathrm{~V}$ and the sample is biased at $-9 \mathrm{~V}$ (See chapter 4.6.5). The current on the sample and on the collector are measured simultaneously to calculate the SEY (Figure 4.8 and 4.9 ).

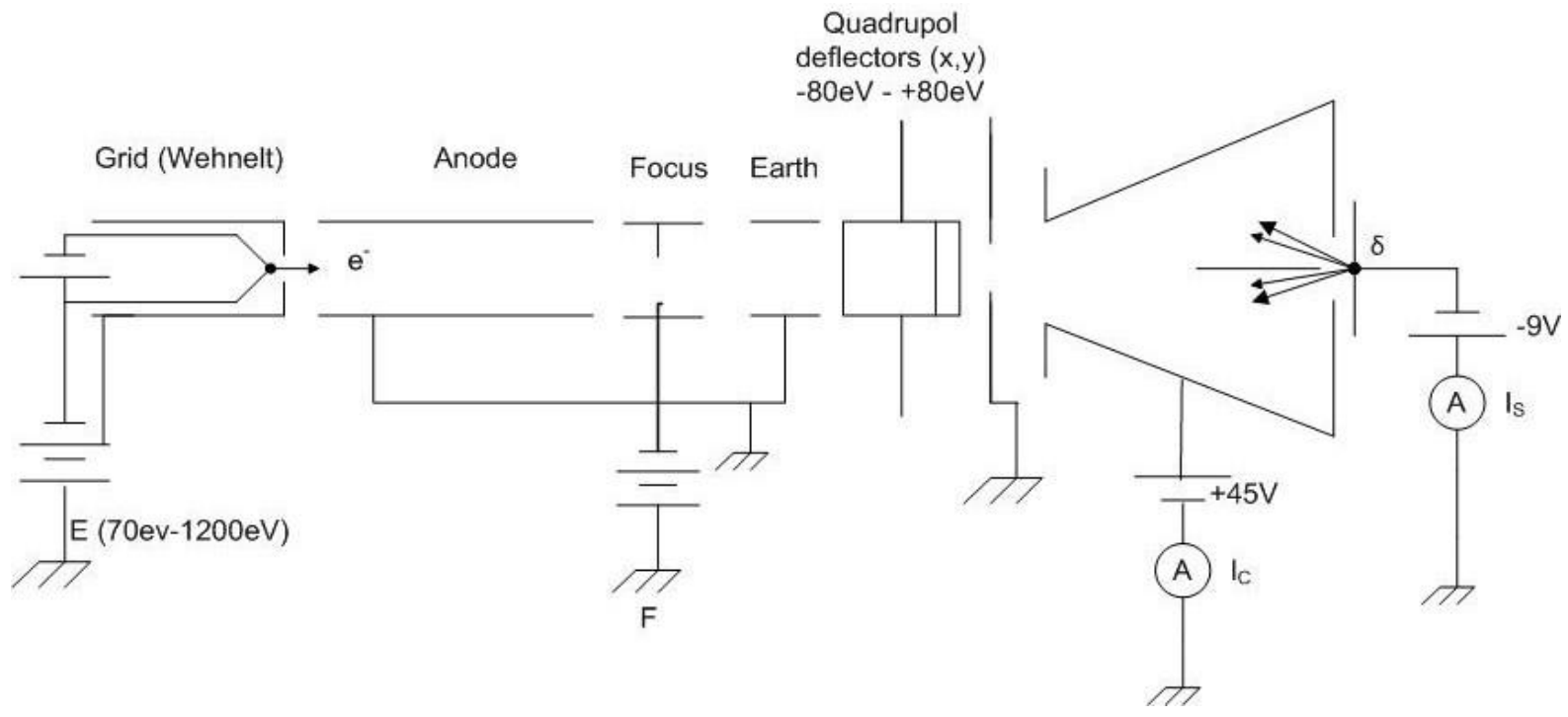

Figure 4.8: The path of electrons starting from the electron gun till the sample. 
Chapter 4- Design of the system for SEY-Measurements at cryogenic temperatures

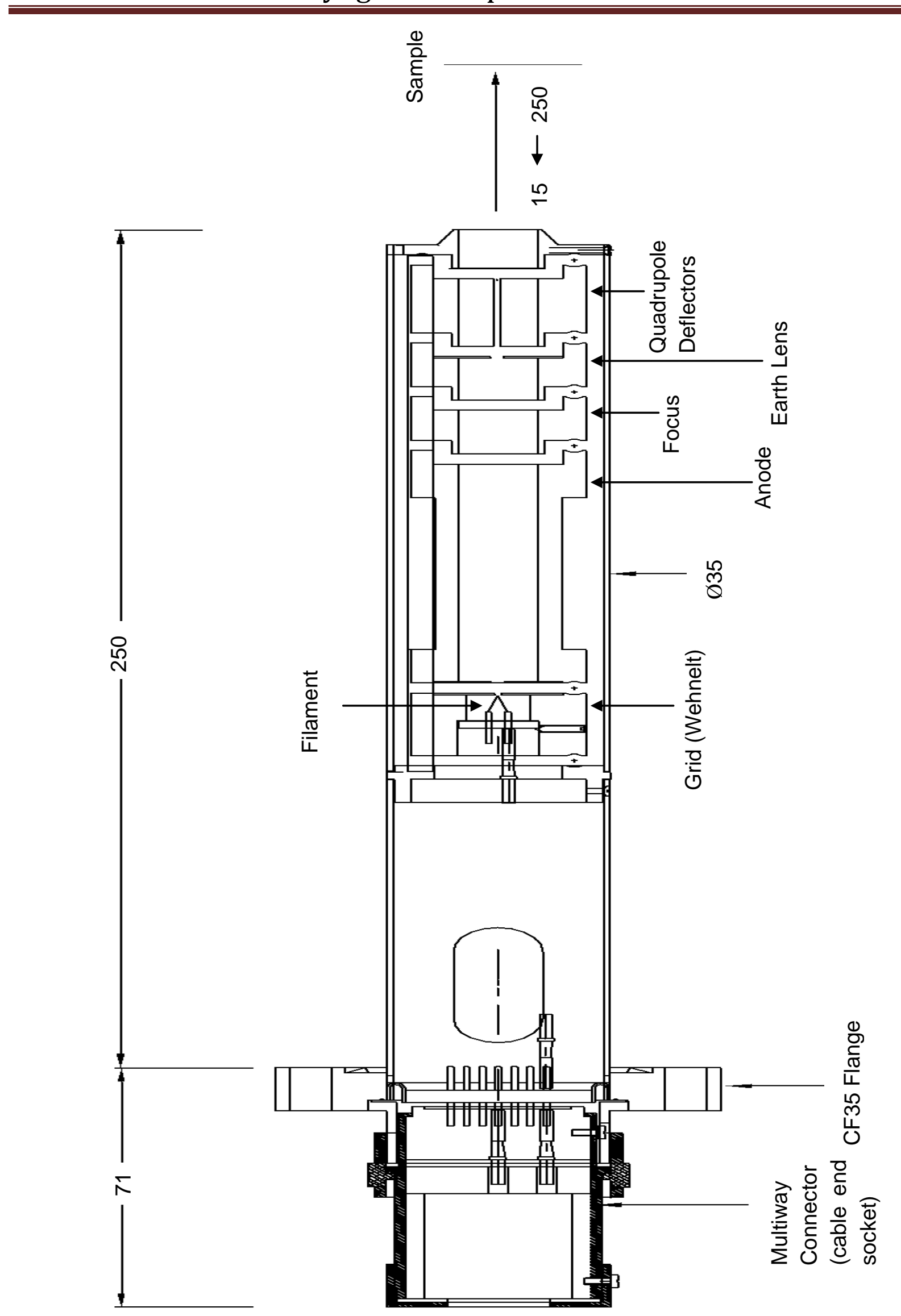

Figure 4.9: Outline drawing of the electron gun. 
A Labview program has been used for the measurements. All the adjustments about the $x-$, $y$-plates and the focussing voltage for each energy are saved on the program. After adjusting the pulse time and the filament current, the sample current and the collector current are read and the yield is calculated.
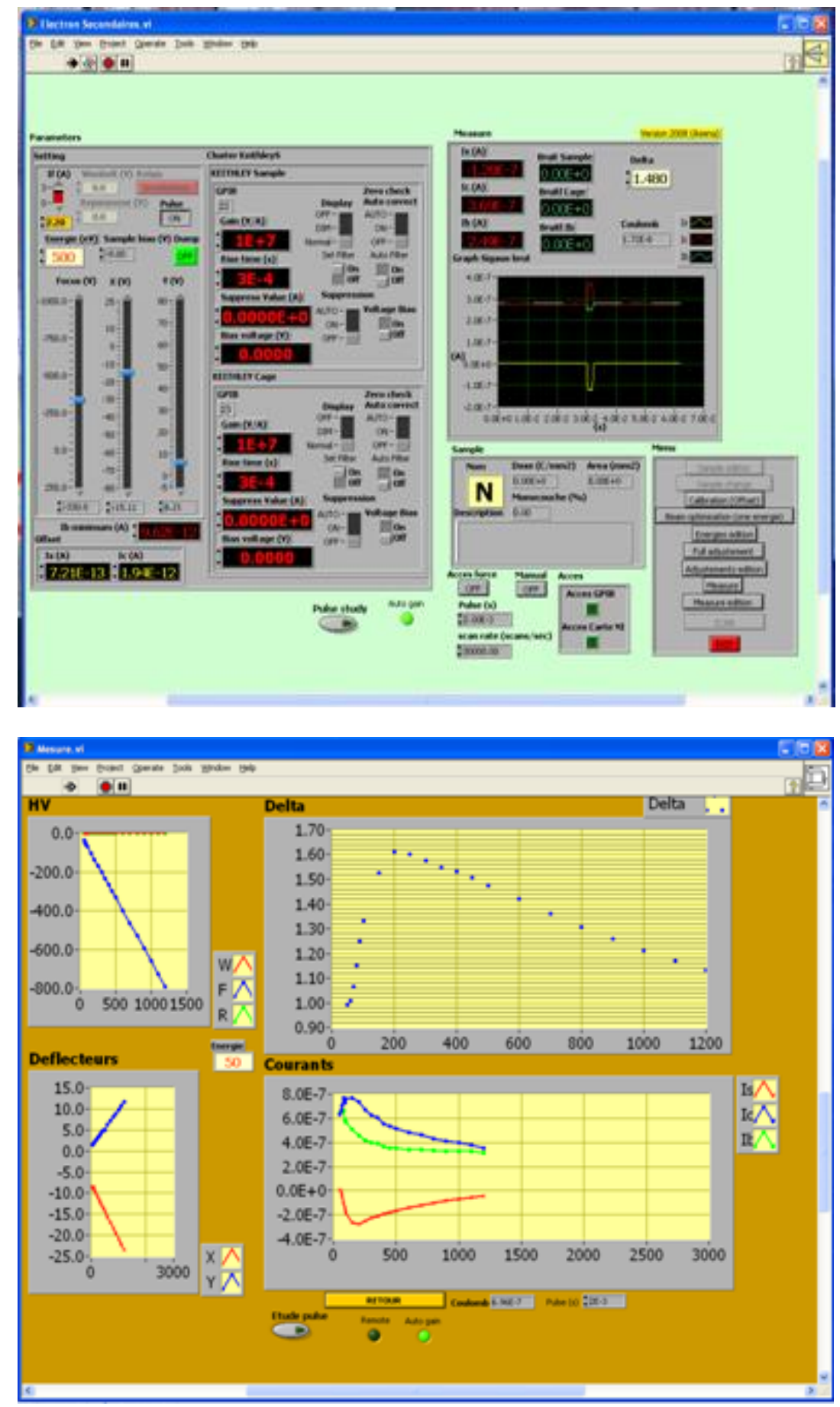

Figure 4.10: Labview programme for SEY measurements. 


\subsection{Commissioning and adjustments of the electron gun parameters}

Before starting the measurements, the electron gun needed to be adjusted and calibrated for the system. Main concerns are the listed below:

- Adjustments of the $x-, y$-deflection plates and the focussing voltage

- Calibration of the beam spot size

- Filament current of the electron gun

- Pulsed vs. continuous beam

The effect of the earth magnetic field on the primary electrons should be considered and the sample bias voltage should be optimised. The results are shown below.

\subsubsection{Calibration of the $x-, y$ - and F- Values}

The new electron gun had to be adjusted, in order to get a well focused and well centered beam on the sample. For that a small mask with a hole of $2 \mathrm{~mm}$ diameter in the center has been placed in front of the sample (Figure 4.11). The beam has been focused in the center of the hole which corresponds to the center of the sample by adjusting the $\mathrm{x}$ and $\mathrm{y}$ plates and the focusing-voltage. In that way, values for $x, y$ and focus has been acquired for some primary energies and these values have been extrapolated for the whole energy range (70ev-1200eV) and used in the software as adjustment of the gun. The calibration values for $\mathrm{X}-, \mathrm{y}-$, and $\mathrm{F}$ are shown in the following graphs (Figure 4.12).

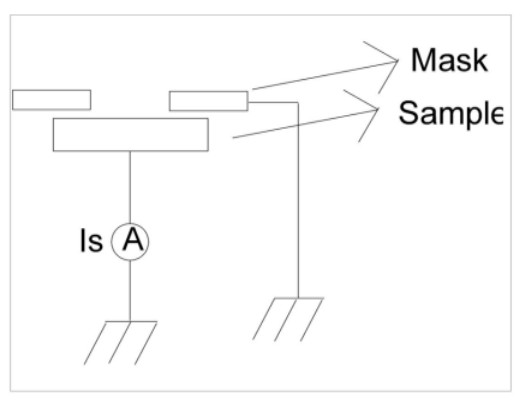

Figure 4.11: The mask with a hole of $2 \mathrm{~mm}$ diameter, replaced in front of the sample without electrical contact to the sample. 
Chapter 4- Design of the system for SEY-Measurements at cryogenic temperatures
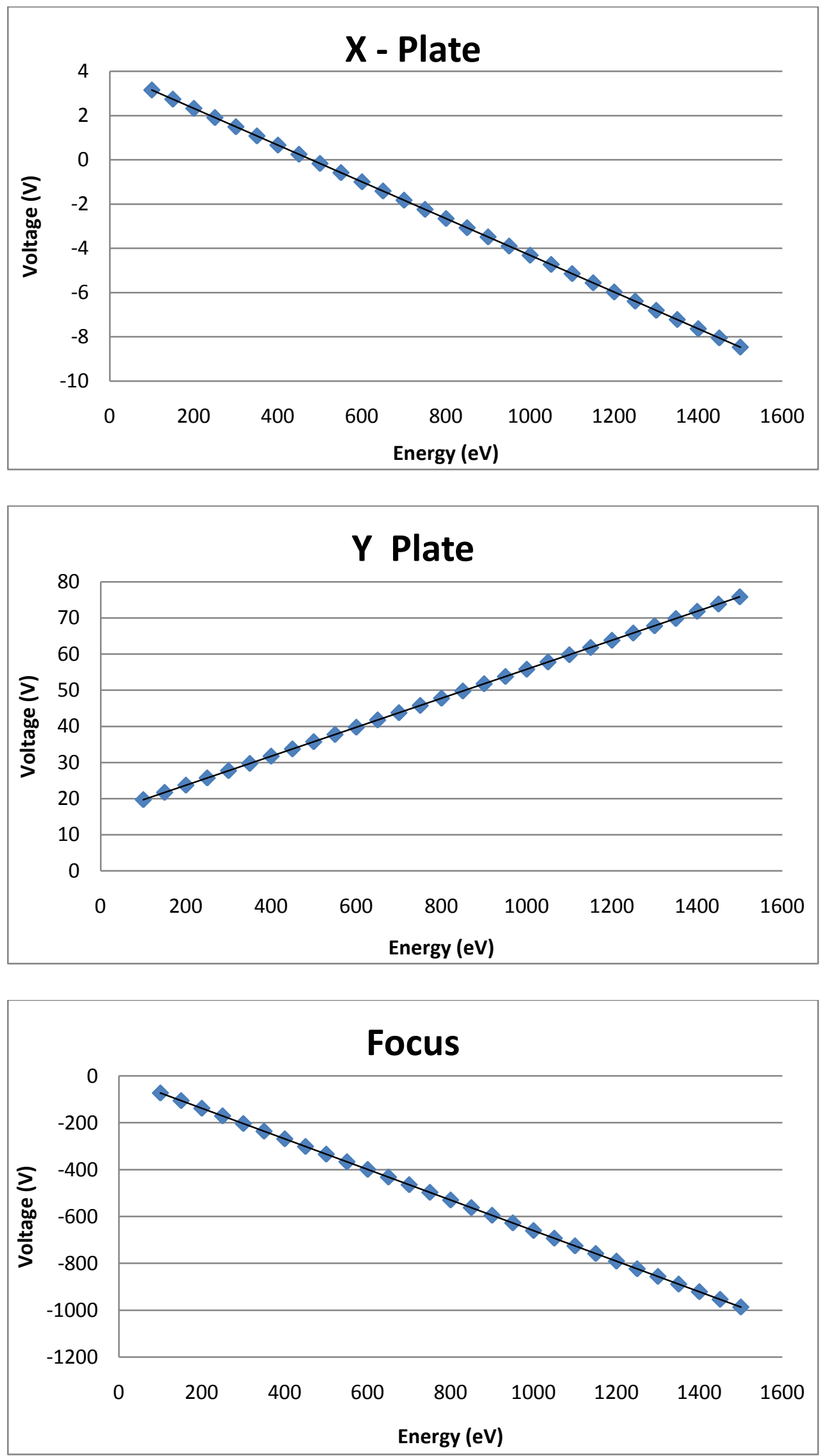

Figure 4.12: The calibration values for $x-, y$-, and $F$. 


\subsubsection{Calibration of the beam spot size}

After scanning the $x, y$ and $F$ values for a defined energy range and calculation of the deflection of the beam for a defined voltage using the the equation $F=q \cdot E$ we could calculate the size of the beam. For that primary electrons with energy of $500 \mathrm{eV}$ were used and 2 different scans were made, once the $x$-value was kept constant and the voltage on the $y$ plate has been changed and afterwards the other way around. The current on the sample has been measured for the different voltages. From the calculated deflection of the beam, the beam size was calibrated. It had a diameter of $1 \mathrm{~mm}$. The scheme of the deflection plates are shown in Figure 4.13. Figure 4.14 shows the Graphs of the current on the sample depending on the voltage on the $x$ - or $y$ - plate.

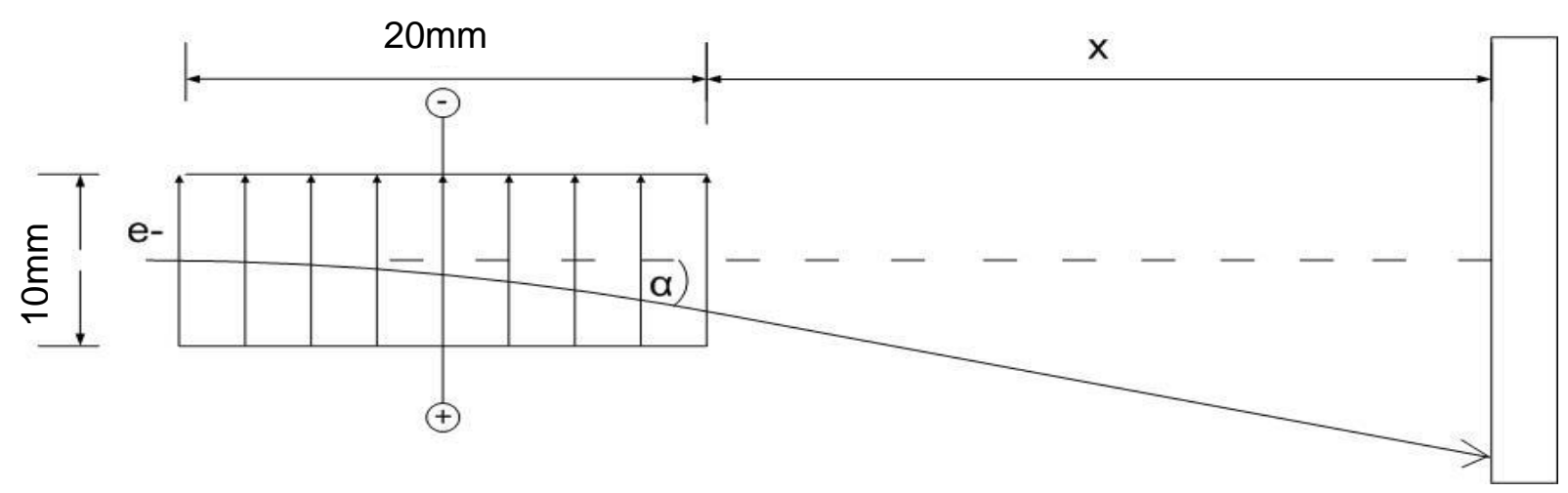

Figure 4.13: The schema of the deflection plate and the path of the electron gun till the sample.

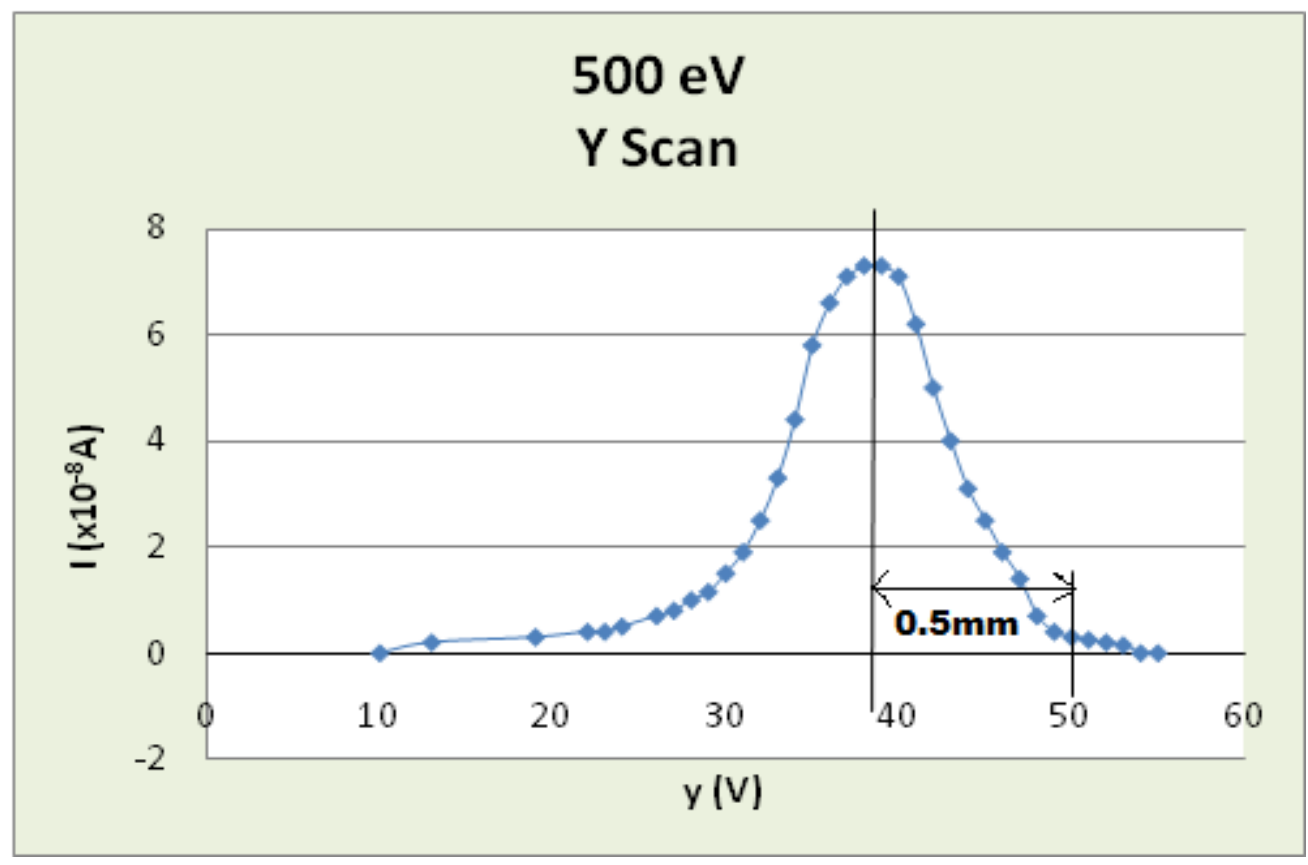




\section{Chapter 4- Design of the system for SEY-Measurements at}

cryogenic temperatures

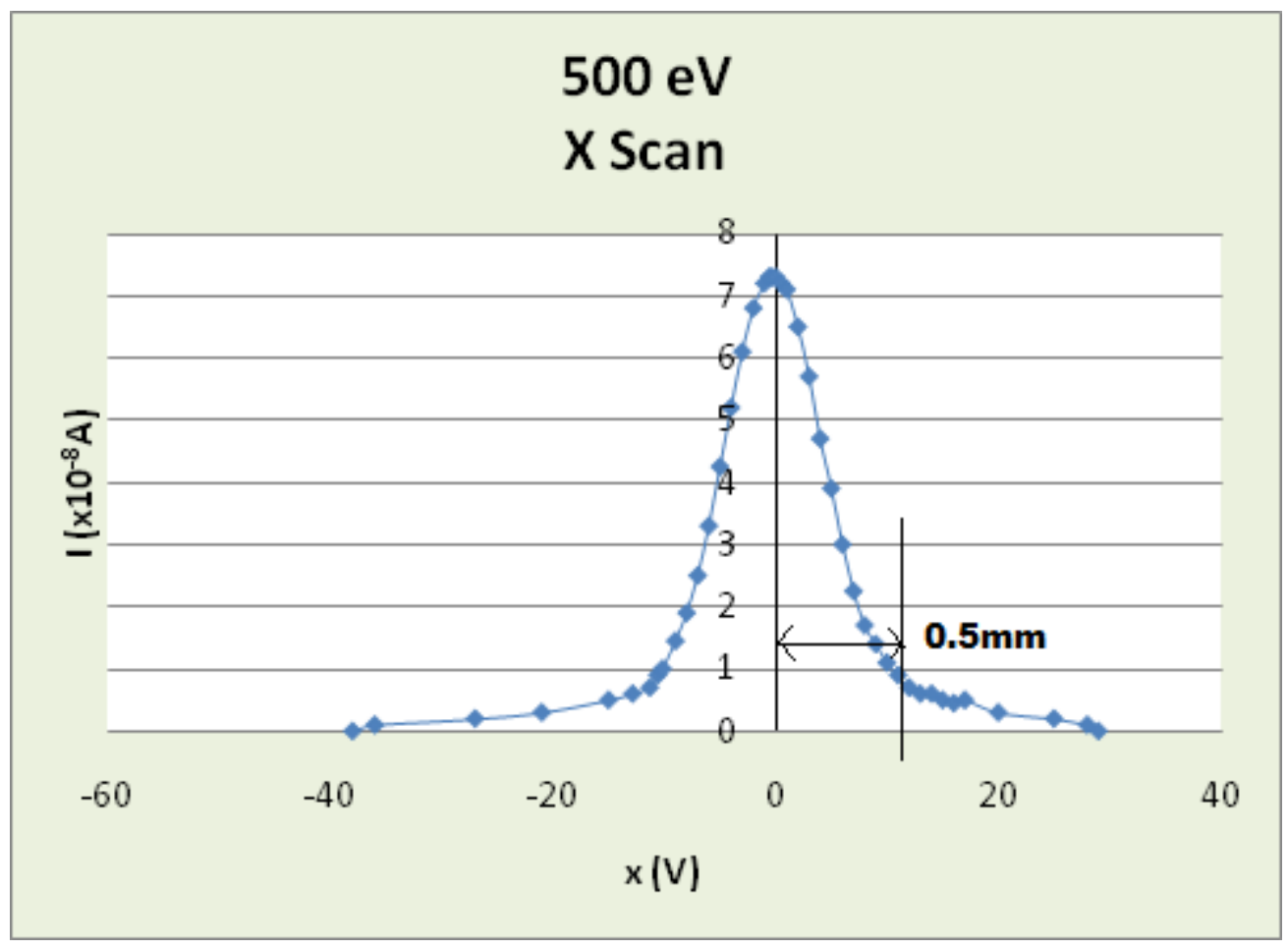

Figure 4.14: Graphs of the current on the sample depending on the voltage on the $x$ - or $y$-plate.

\subsubsection{Filament current of the electron gun}

For SEY measurements, it is important to minimize the dose on the sample to avoid desorption of the adsorbed gases. In order to minimize the dose on the sample, it is necessary to keep the beam current low. For that purpose measurements with different filament currents of the gun have been performed, to find out the lowest possible beam current, which still allows to perform the SEY measurements. Three different filament currents have been measured: 2.2A, 2.1A and 2.05A. The results show that the yield doesn't change for these three different currents but the beam current decreases with decreasing filament current. The less the beam current the less is the current on the sample and accordingly the less is the dose on the sample. The results are shown in Figure 4.154 .16 and 4.17. For measurements a filament current of $2.1 \mathrm{~A}$ have been used. 
Chapter 4- Design of the system for SEY-Measurements at cryogenic temperatures

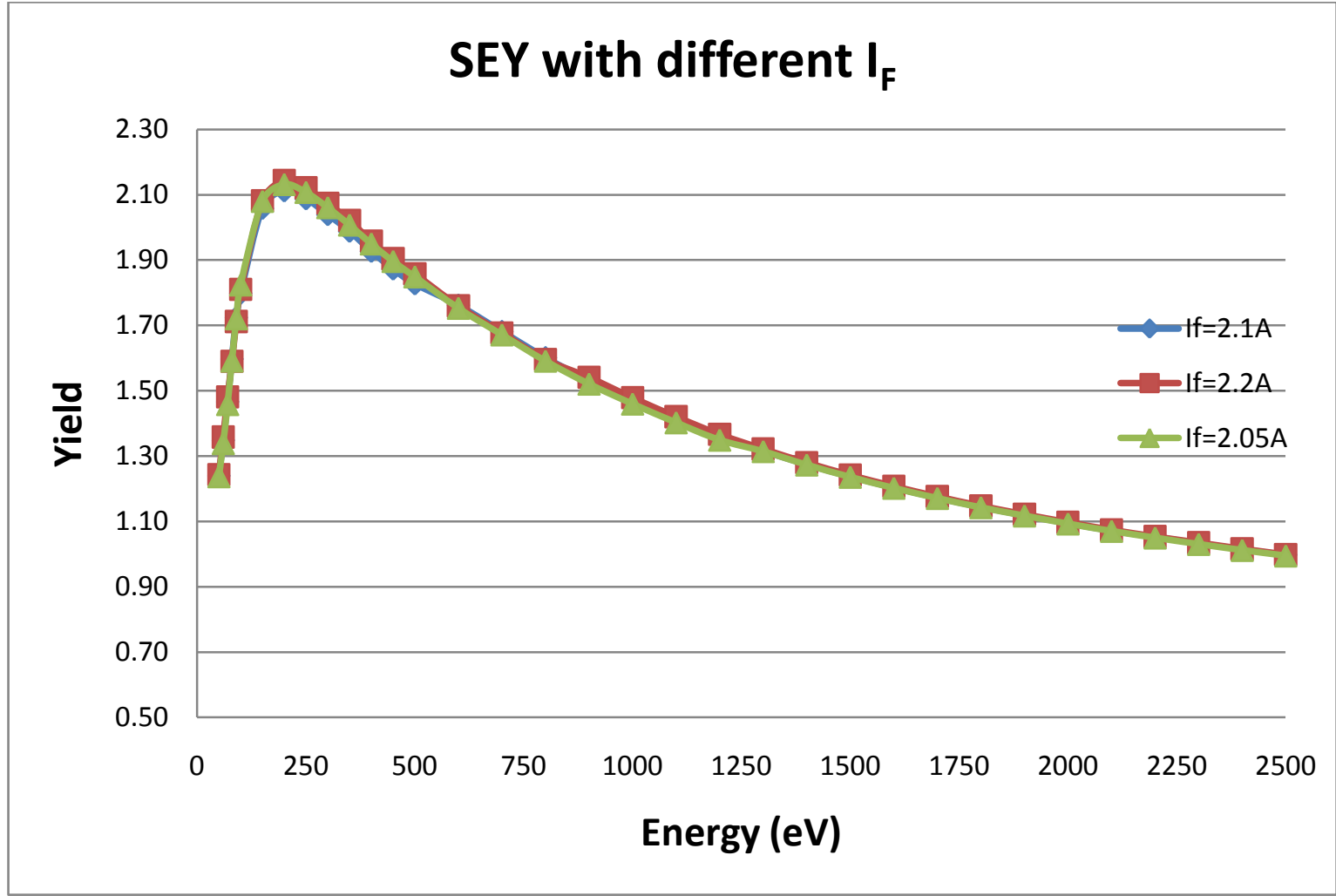

Figure 4.15: Secondary electron yield as a function of filament current on the electron gun.

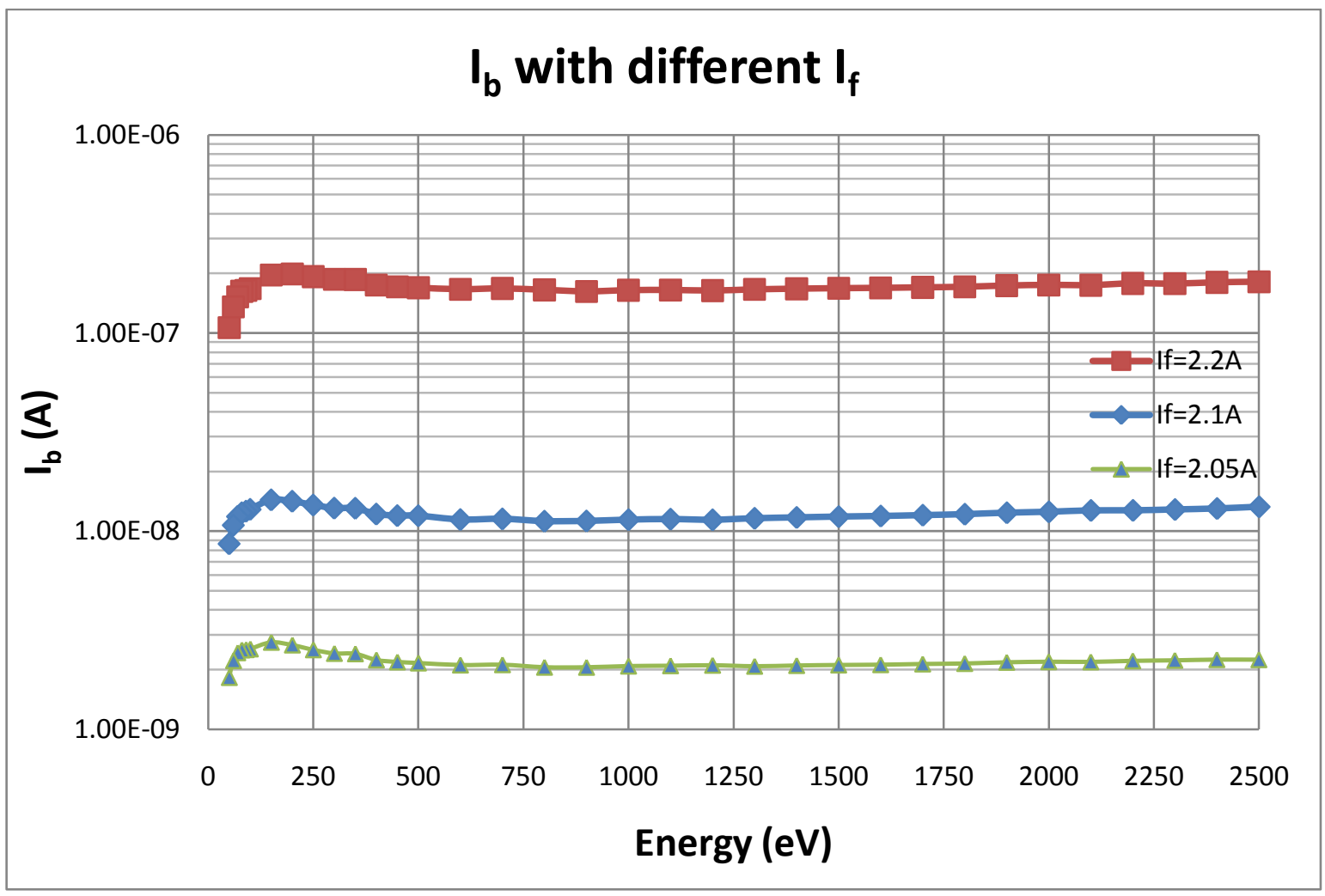

Figure 4.16: Total beam current as a function of filament current on the electron gun. 
Chapter 4- Design of the system for SEY-Measurements at cryogenic temperatures

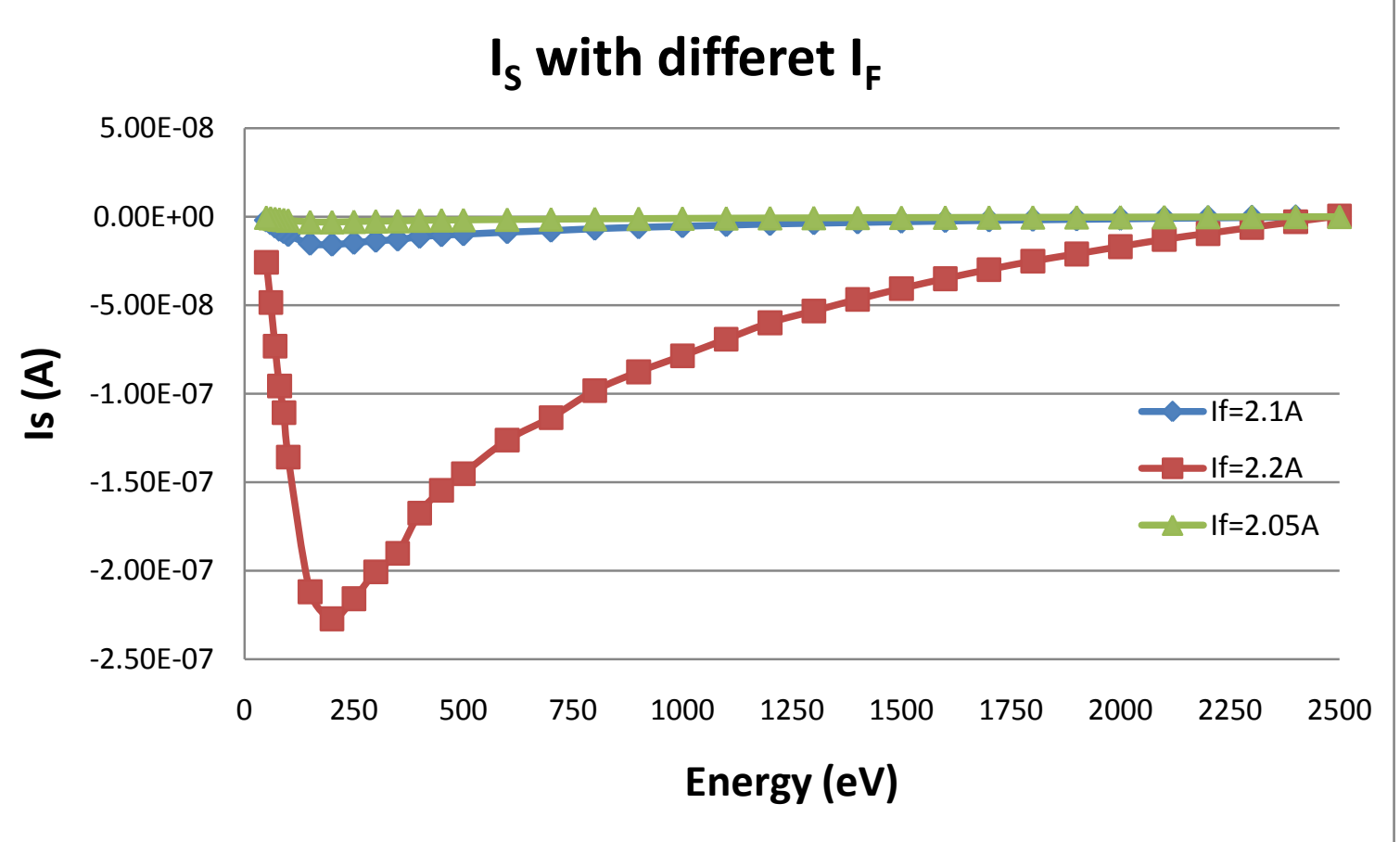

Figure 4.17: Sample current as a function of filament current on the electron gun.

\subsubsection{Calculation of the magnetic field effect on the primary electrons}

To estimate the effect of the magnetic field of the earth on the primary electrons coming out of the gun, some calculations have been done. For the calculation the magnetic field of the earth has been set as $3 \times 10^{-5} \mathrm{~T}$, the angle between the $\mathrm{v}_{\mathrm{e}}$ and $\mathrm{B}$ as $10^{\circ}$ and the distance from the end of the gun till sample is $130 \mathrm{~mm}$.

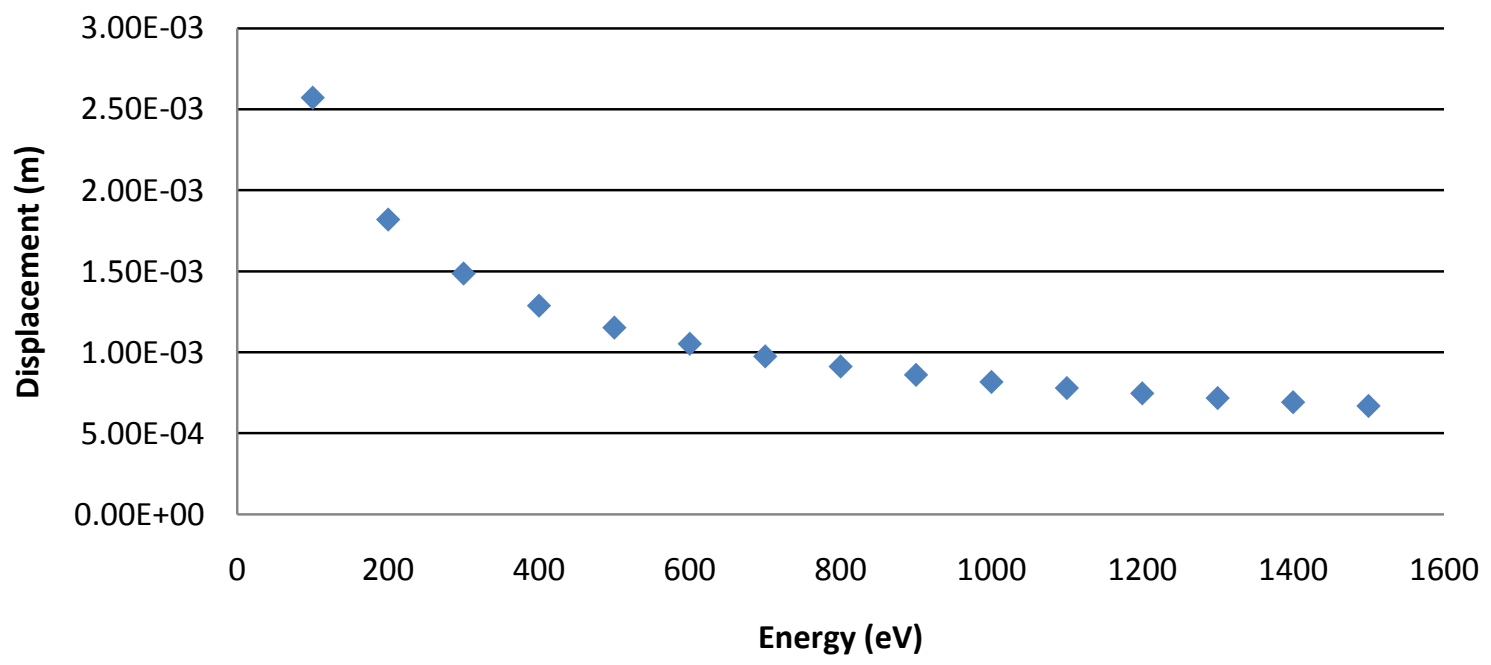

Figure 4.18: Displacement of the electrons with the earth magnetic field as a function of their energy. 


\section{Chapter 4- Design of the system for SEY-Measurements at cryogenic temperatures}

It is easy to see that the earth magnetic field displaces the electrons especially the ones with low energy. To reduce that effect the system has been oriented in the direction of the earth magnetic field. Also the magnet on the penning gauge affects the electrons trajectories. To avoid this effect the magnet on the gauge has been removed before the measurements.

\subsubsection{Sample bias}

A bias voltage on the sample is necessary in order to make the secondary electrons leave the sample surface. To understand the effect of the sample bias, measurements with different voltages have been done. With zero bias, the number of electrons hitting the surface and the number of electrons leaving the surface are the same so that the yield is 1 but for a wide range of bias voltages the measured SEY does not change. Figure 4.19 shows the results. The following measurements were performed with a sample bias of $-9 \mathrm{~V}$.

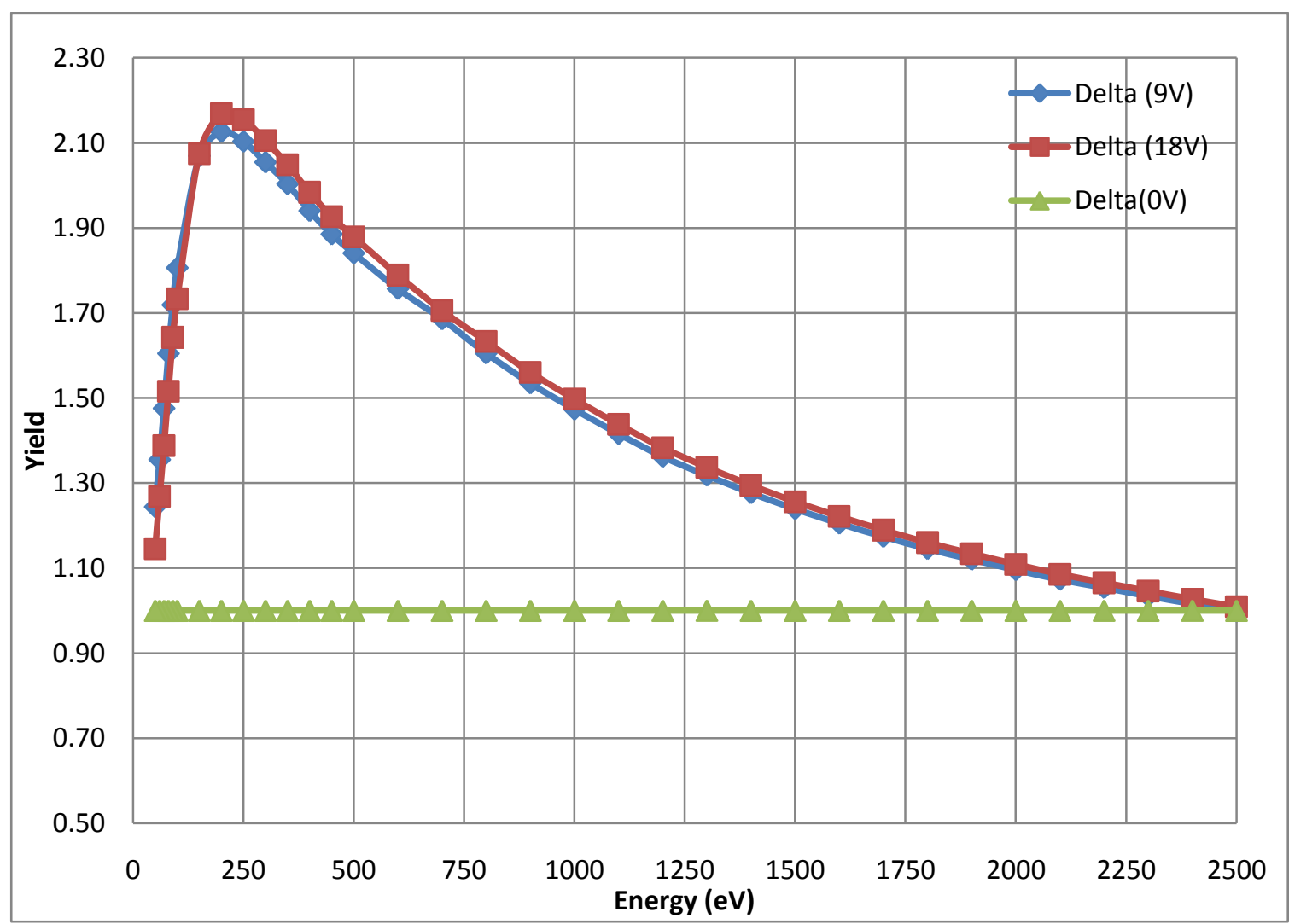

Figure 4.19: Secondary electron yield as a function of the negative bias voltage. 


\section{Chapter 4- Design of the system for SEY-Measurements at cryogenic temperatures}

\subsubsection{Pulsed beam}

To keep the electron dose on the sample at minimum is important in order to avoid the charging of the sample, not to do conditioning of the sample and to avoid desorption of the condensed gases. The following results show that the electron dose with pulsed beam is high enough to perform the SEY measurements like with continuous beam but with pulsed beam the electron dose on the sample is low. To find the minimum pulse time, with which the electron dose is still enough to perform the SEY measurements, measurements with different pulse times have been performed. It is to see that SEY measurements are still possible with $2 \mathrm{~ms}$ pulse time (Figure 4.20 ) therefore all the measurements were performed with $2 \mathrm{~ms}$ pulse time.
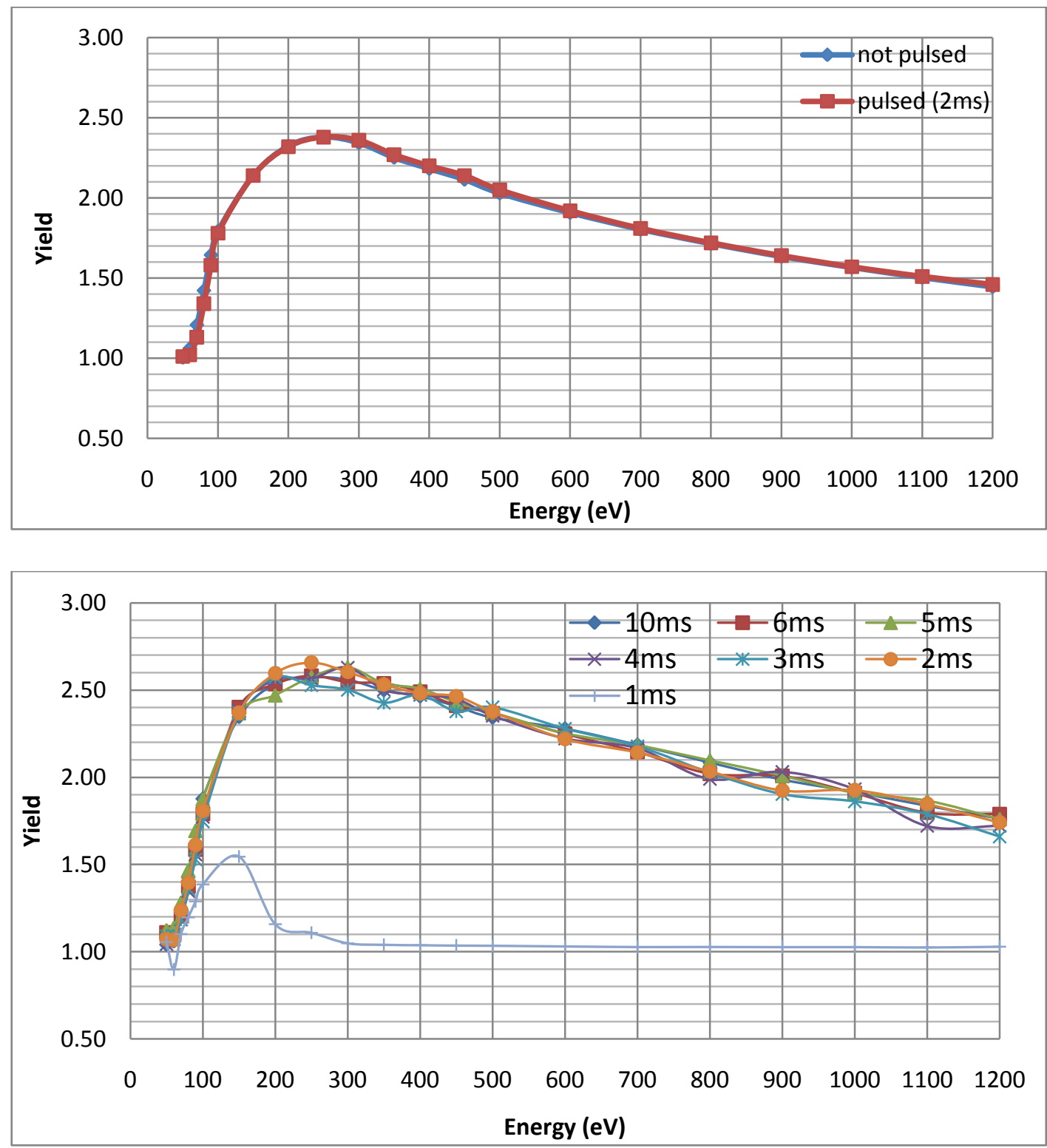

Figure 4.20: SEY for pulsed and continues beam and investigations of different pulse times. 


\section{Chapter 4- Design of the system for SEY-Measurements at cryogenic temperatures}

In order to verify that the electron dose on the sample with $2 \mathrm{~ms}$ pulse time is low enough to avoid the desorption of the condensed gases, the number of monolayers of $\mathrm{N}_{2}$ desorbed by that electron dose has been calculated by using the reference [47]. Figure 4.21 shows the desorption yield of $\mathrm{H}_{2}, \mathrm{CH}_{4}, \mathrm{CO}, \mathrm{N}_{2}$ and $\mathrm{CO}_{2}$ as a function of the electron energy calculated by [47].

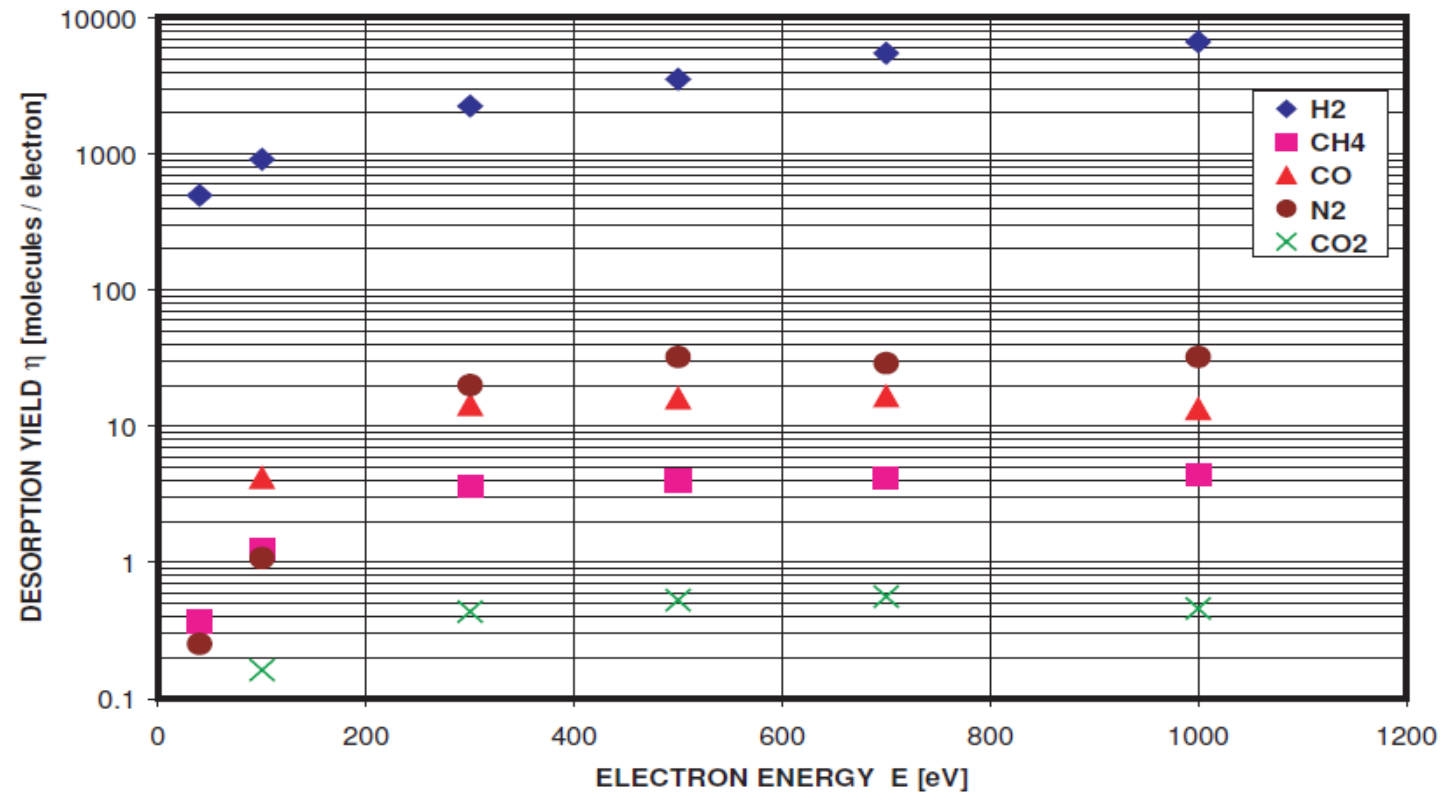

Figure 4.21: The desorption yield of $\mathrm{H}_{2}, \mathrm{CH}_{4}, \mathrm{CO}, \mathrm{N}_{2}$ and $\mathrm{CO}_{2}$ as a function of the electron energy [47].

The number of electrons coming out of the electron gun during $2 \mathrm{~ms}$ pulse is:

$$
I=\frac{e . N}{t} \quad \rightarrow \quad N=\frac{\text { I.t }}{e}=1.2510^{9} \text { electrons }
$$

where $\mathrm{E}$ is $500 \mathrm{eV}, \mathrm{I}$ is $1 \cdot 10^{-7} \mathrm{~A}$ and $\mathrm{t}$ is $2 \cdot 10^{-3} \mathrm{~s}$. The duration of one measurement is $30 \mathrm{~s}$.

Using the Figure 4.6 one electron with $500 \mathrm{eV}$ can desorb app. 30 molecules of $\mathrm{N}_{2}$, which means $1.25 \cdot 10^{9}$ electrons with $500 \mathrm{eV}$ can desorb $3.75 \cdot 10^{10} \mathrm{~N}_{2}$ molecules or $3.75 \cdot 10^{-5}$ monolayers of $\mathrm{N}_{2}$. By using the continuous beam with the same conditions, the number of desorbed $\mathrm{N}_{2}$ molecules during one measurement is about $2 \cdot 10^{-2}$ monolayers.

The amount of monolayers desorbed with the pulsed electron beam is negligible for the SEY measurements. 


\section{Chapter 4- Design of the system for SEY-Measurements at cryogenic temperatures}

\subsection{Cryogenic measurements}

\subsubsection{Why cryogenic measurements of SEY?}

In physics, the word cryogenics is used as a synonym for low temperatures below $\quad-150^{\circ} \mathrm{C}$. The word cryogenics stems from Greek and means 'the production of freezing cold'. Besides the production of very low temperatures, it also studies the behaviour of materials at those temperatures. In cryogenics it is common to use the Kelvin-scale. Cryogenics involves different areas of engineering; Rocket propulsion systems, Studies in high-energy physics, Electronics, Mechanical design, Space simulation and high-vacuum technology, Superconductivity, Biological and medical applications, Food processing, Manufacturing processes, Recycling of materials...

There are several significant effects that appear only at very low temperatures, like vanishing of specific heats, ductile-brittle transitions in carbon steel and superconductivity. Superconductivity is the simultaneous disappearance of all electric resistance and the appearance of perfect diamagnetism. In the absence of a magnetic field, many elements, alloys, and compounds become superconducting at a well-defined temperature, called the transition temperature in zero field $t_{0}$. Superconductivity can be destroyed by increasing the magnetic field around the material to a sufficiently large value.

In order to have a high energy beam with a fixed bending radius, the most important technologies for the construction of CERN were high-field superconducting magnets and superfluid helium cryogenics. LHC has 1750 main superconducting magnets and 8000 superconducting correctors. In total in the LHC some $15 \mathrm{GJ}$ of magnetic energy will be stored in superconductive magnets. In order to keep the magnets in superconducting state, some parts of the LHC is at cryogenic temperatures.

To understand the electron-cloud-effect in the cryogenic parts of the LHC, the SEY measurements at cryogenic temperatures were performed. To achieve the very low temperatures, a cold head has been used, which was manufactured in the Cryolab of CERN.

As a cryogenic fluid we used Helium. Helium has two stable isotopes: $\mathrm{He}^{4}$, the most common one, and $\mathrm{He}^{3}$. Ordinary helium gas contains about $1.3 \times 10^{-4}$ percent $\mathrm{He}^{3}$, so when speaking of helium or liquid helium, it is referred to $\mathrm{He}^{4}$, unless otherwise stated. Liquid $\mathrm{He}^{4}$ has a normal boiling point of $4,214 \mathrm{~K}$ and a density at the normal boiling point of 124,8 


\section{Chapter 4- Design of the system for SEY-Measurements at cryogenic temperatures}

$\mathrm{kg} / \mathrm{m}^{3}$. Liquid helium has no freezing point at a pressure of $1 \mathrm{~atm}$. In fact, liquid helium does not freeze under its own vapour pressure even if the temperature is reduced to absolute zero.

\subsubsection{Physical quantities for a cryogenic system}

\section{I. $\quad$ Thermal conductivity}

The thermal conductivity $k_{t}$ of a material is defined as the heat-transfer rate per unit area divided by the temperature gradient causing the heat transfer. There are three mechanisms responsible for conduction of heat through materials: (1) electron motion, as in metallic conductors; (2) lattice vibrational-energy transport or phonon motion, as in all solids and liquids; (3) molecular motion, as in organic solids and gases [46]. The thermal conductivity $\mathrm{k}_{\mathrm{t}}$ is determined as following:

where

$$
k_{t}=\frac{n c_{v}\langle v\rangle \lambda}{3 N_{A}}
$$

n ... molecules per unit volume

$\mathrm{C}_{\mathrm{v}} \quad \ldots$ molar heat capacity

$\langle v\rangle \ldots$ mean molecular sped

$\lambda$... mean free path

$\mathrm{N}_{\mathrm{A}}$....Avogadro's number

A decrease in temperature results in a decrease in the mean molecular speed and consequently, a decrease in the gas thermal conductivity.

Energy is transported in metals by electronic and phonon motion. The thermal conductivity increases to a very high maximum as the temperature is lowered, until the mean free path of the energy carriers becomes on the order of the dimensions of the material sample. When this condition is reached, the boundary of the material begins to introduce a resistance to the motion of the carriers, and the carrier mean free path becomes constant (approximately equal to the material thickness). Because the specific heat decreases to zero as the absolute temperature approaches zero, we see that the thermal conductivity would also decrease with a decrease in temperature in this very low temperature region. The thermal conductivity of alloys and impure metals decrease as the temperature is decreased. 


\section{Chapter 4- Design of the system for SEY-Measurements at cryogenic temperatures}

\section{Electrical conductivity}

The electrical conductivity $k_{e}$ of a material is defined as the electric current per unit crosssectional area divided by the voltage gradient in the direction of current flow. When an external electric filed is applied to an electric conductor, free electrons in the conductor are forced to move in the direction of the applied field. This motion is opposed by the positive ions of the metal lattice and impurity atoms present in the material. Decreasing the temperature of the conductor decreases the vibrational energy of the ions, which in turn results in a smaller interference with electron motion. Therefore, the electrical conductivity increases as the temperature is lowered for metallic conductors.

Drude has developed the first theory of electric resistance through treating the free electrons as an 'electron gas'. He obtained the following expression [46]:

$$
k_{e}=\frac{(N / V) e^{2} \lambda}{m_{e}\langle v\rangle}
$$

where N/V...number of free electrons per unit volume

e ... electron charge

$\lambda$... mean free path of an electron

$\mathrm{m}_{\mathrm{e}}$...mass of an electron

$\langle v\rangle_{\ldots}$ mean electron speed

\section{Heat radiation}

A cold sample can be heated by radiation and this power must be dissipated by cooling. The thermal radiation power is given:

$$
P=\sigma \cdot A \cdot E \cdot\left(T_{1}^{4}-T_{2}^{4}\right)
$$

E... Radiation exchange grade

$$
E:=\frac{1}{\frac{1}{\varepsilon_{1}}+\frac{1}{\varepsilon_{2}}-1}
$$

$\varepsilon_{1}, \varepsilon_{2} \ldots$ Emission coefficient of different plates 


\section{Chapter 4- Design of the system for SEY-Measurements at cryogenic temperatures}

\subsubsection{Principles of the cold head}

The cold head is shown in the photograph in Figure 4.22, 4.23 and schematically in Figure 4.24. The system is working in horizontal position.

For cooling, liquid helium coming from the transfer line arrives into the phase separator. Gas produced by this transfer feeds a heat exchanger which cools down a thermal screen at approximately $30 \mathrm{~K}$ (red circuit). Liquid from the phase separator is transferred through an expansion valve to a double wall exchanger.

The primary function of the heat exchanger is to allow the removal of energy from the compressed incoming gas by transferring energy to the low-pressure cold return gas. The liquid helium flow and a heater around the sample permit us to regulate the temperature of the sample.
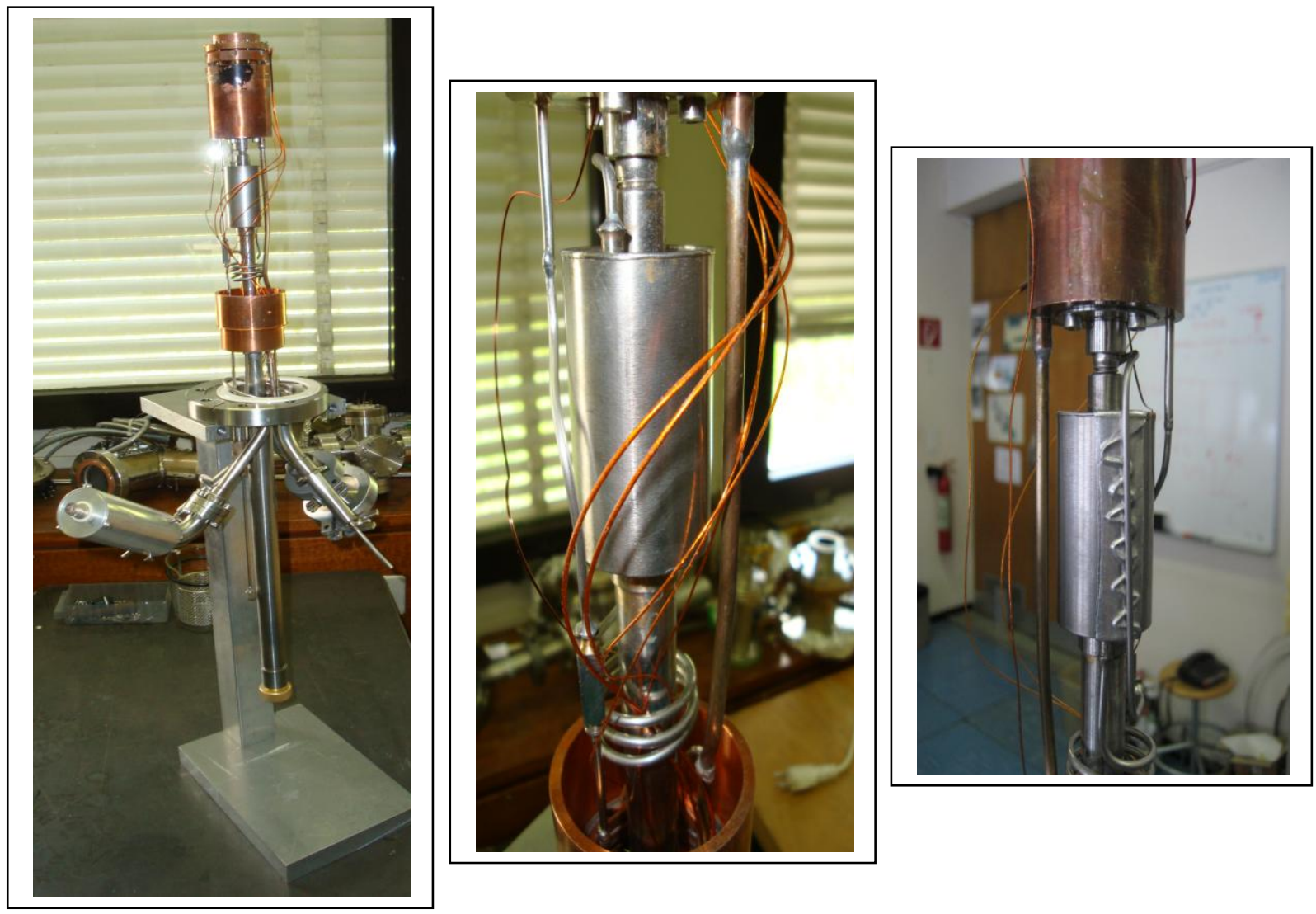

Figure 4.22: Cold head with wirings of the silicon-diode thermometer and the heater. Third photo after thermolizing the diode wires. 
Chapter 4- Design of the system for SEY-Measurements at cryogenic temperatures

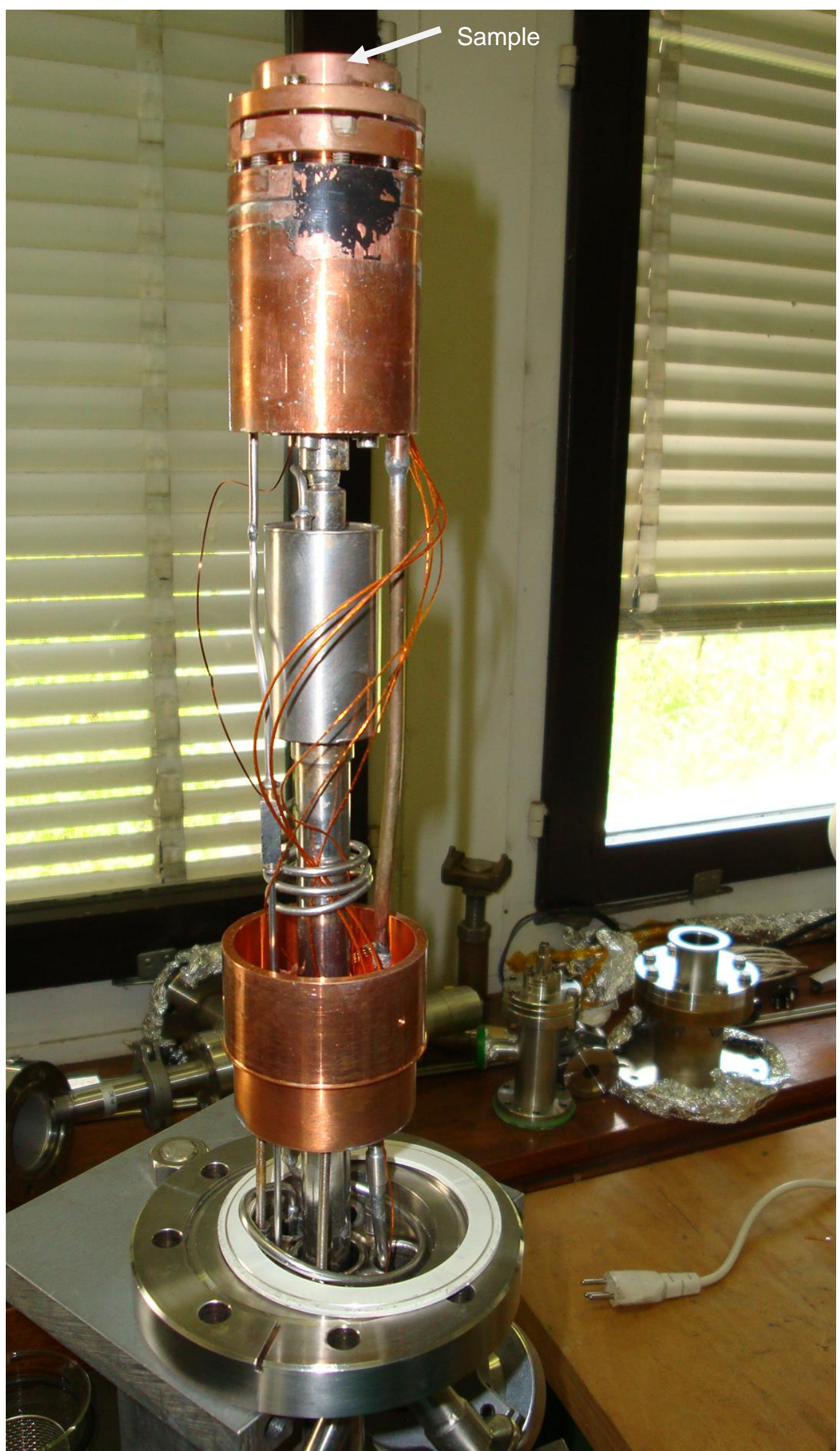

Figure 4.23: Cold head with dismounted thermal shield. 


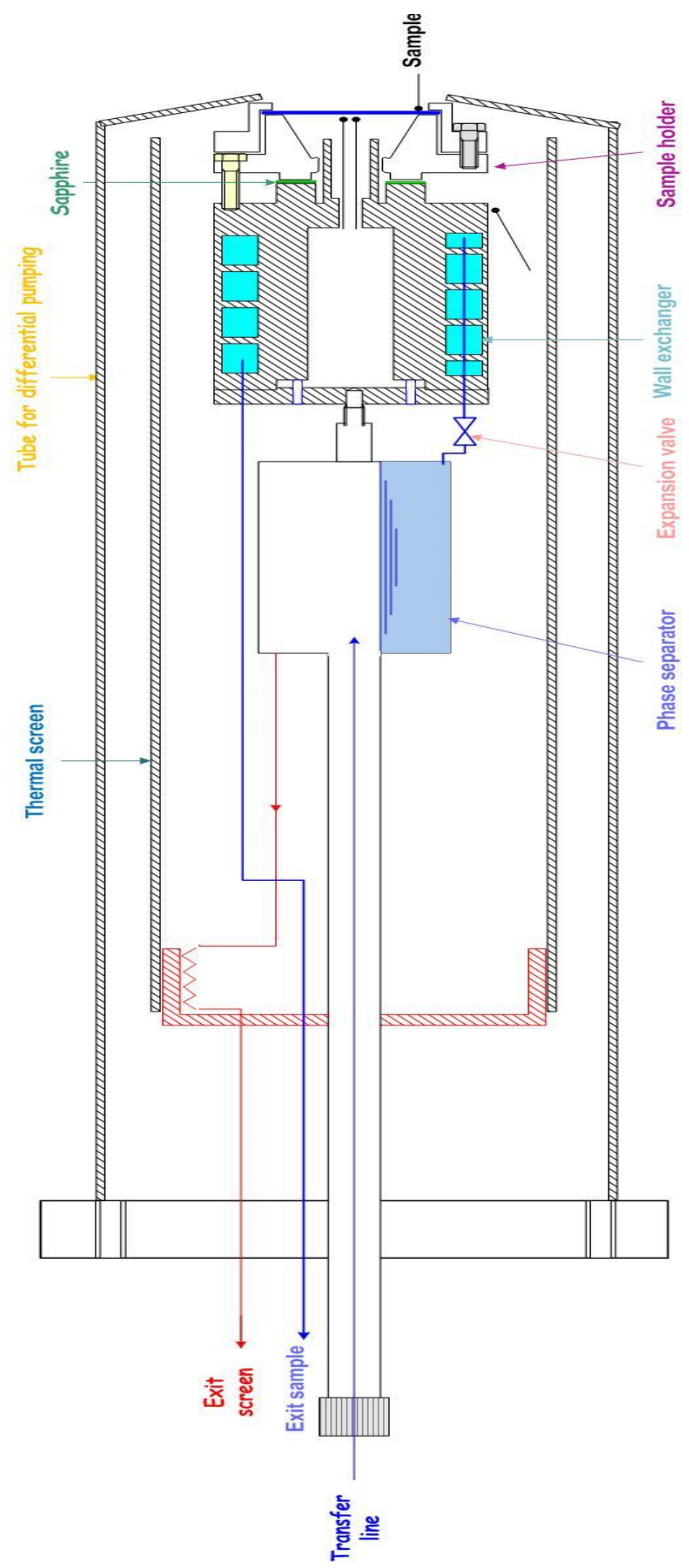

Figure 4.24: Piping \& Instrumentation Diagram of a cryostat for a cold head at variable temperature. 


\section{Chapter 4- Design of the system for SEY-Measurements at cryogenic temperatures}

The sample is fixed with aluminium bolts $(M 3 \times 6)$ on a copper support, the sample holder (Figure 4.25). This sample holder is connected to the cold source via a sapphire ring with polished faces, of an external diameter $28 \mathrm{~mm}$, internal diameter $15 \mathrm{~mm}$, thickness $0.5 \mathrm{~mm}$, i.e., a contact surface of $4.39 \mathrm{~cm} 2$. At either side of the sapphire ring, an indium wire makes a cold weld to the cold head and the sample holder, maintained mechanically in place by insulating screws in PEEK (4xM6). The sample is thus electrically insulated with respect to the cold source, but in excellent thermal contact.

A silicon diode thermometer has been glued on the sapphire in contact with the sample to measure the temperature difference between the copper plates constituting the contact. The thermometer is glued on the sapphire by using special glue called Stycast 2850 FT because of its low outgassing rate. The wire of the diode has been thermalized by clamping it via indium foil on the phase separator. During measurements, the temperature of the sample reaches $4.7 \mathrm{~K}$. On the inner part of the sample holder, as close as possible to the sample, a rectangular thermofoil heater with kapton insulation has been glued. We used again the same glue as for thermometer.

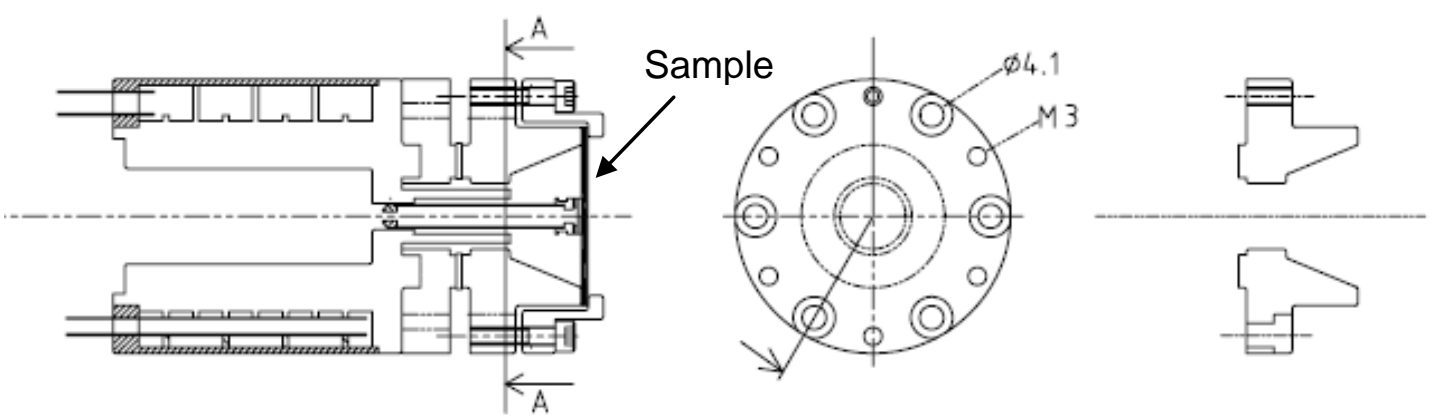

Figure 4.25: Mechanical drawing of the sample holder.

To be able to make the differential pumping a tube has been designed to be mounted around the cold head (Figure 4.24). Stainless steel and kapton disks are mounted above the sample holder to cover the top part of the cold head, in order to perform differential pumping.

The cold head has been mounted on a bellow, in order to change the distance between the sample and the collector. 


\subsubsection{Cooling procedure and performance}

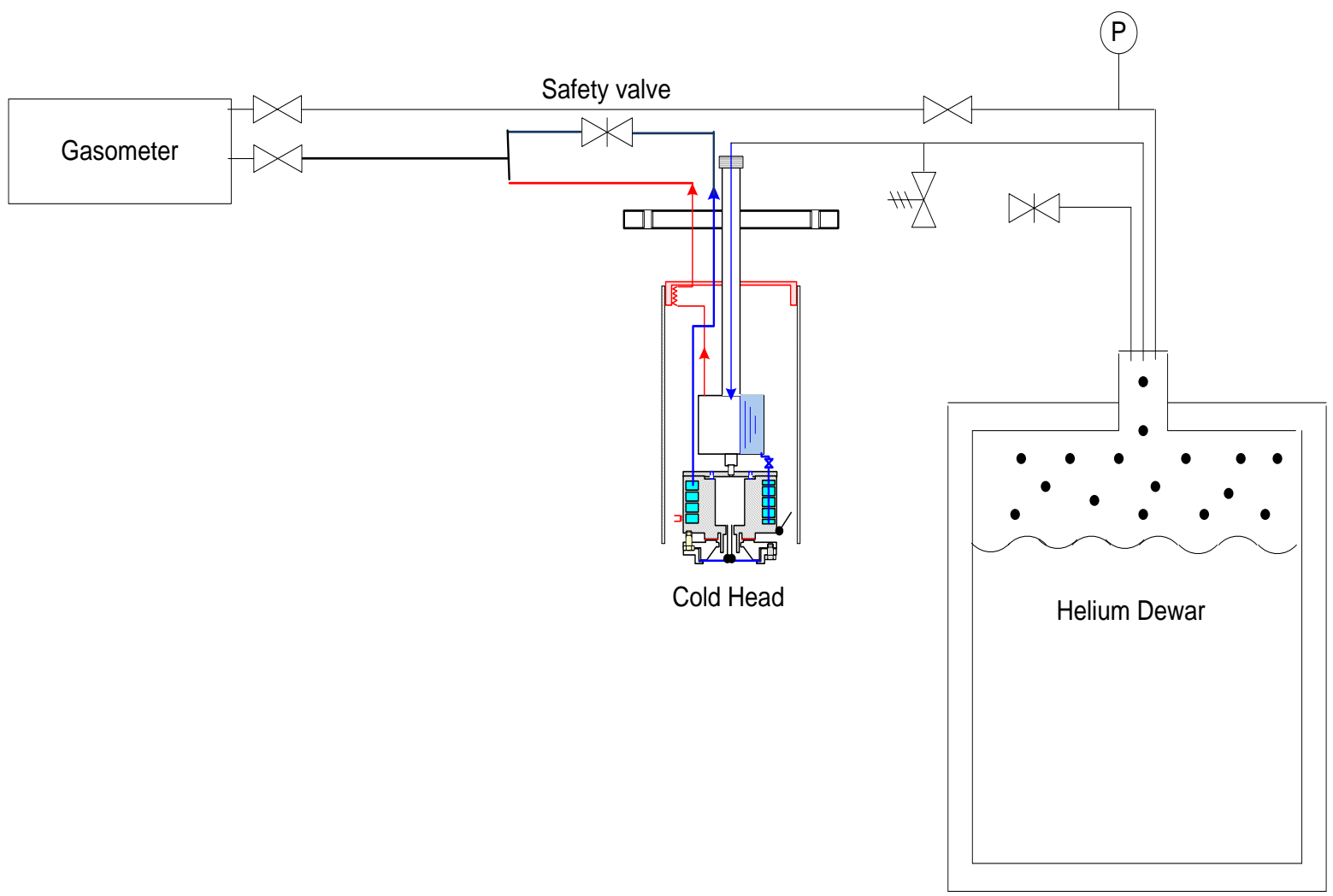

Figure 4.26: The connections of the cold head with the gasometer and the helium Dewar.

Before starting cooling, the cold head has to be flushed to get rid of oxygen. For that Nitrogen from a bottle will be let into the helium line of the cold head and right afterwards the helium line of the cold head will be pumped again.

To start with the cold measurements, the cold head has to be connected to a Dewar, which provides the liquid helium for cooling, and with the gasometer, to send back the helium gas to the Cryolab. The cold head has two outlets and both of them are connected to the gasometer via a long tube. To assemble that tube to the outlet, helicoflex seals were used, because they can stand the cryogenic temperatures. One of the outlets has a safety valve, to protect the system from explosions in case of overpressure.

The cold head and the helium Dewar are interconnected through a transfer line. The transfer line has to be connected to the Dewar before being connected to the cold head. Just after 


\section{Chapter 4- Design of the system for SEY-Measurements at cryogenic temperatures}

having seen that the liquid helium is coming out of the transfer line, the transfer line can be placed in the cold head.

To be able to transfer liquid helium from the Dewar to the cold head, the gas pressure in the Dewar has to be increased. For that purpose, helium gas from a helium bottle will be let in the helium Dewar. While cooling down, the valve going from the Dewar to the gasometer is closed, to preserve the high pressure. After ending the measurements, we have to open the valve between Dewar and gasometer to release the pressure in the Dewar.

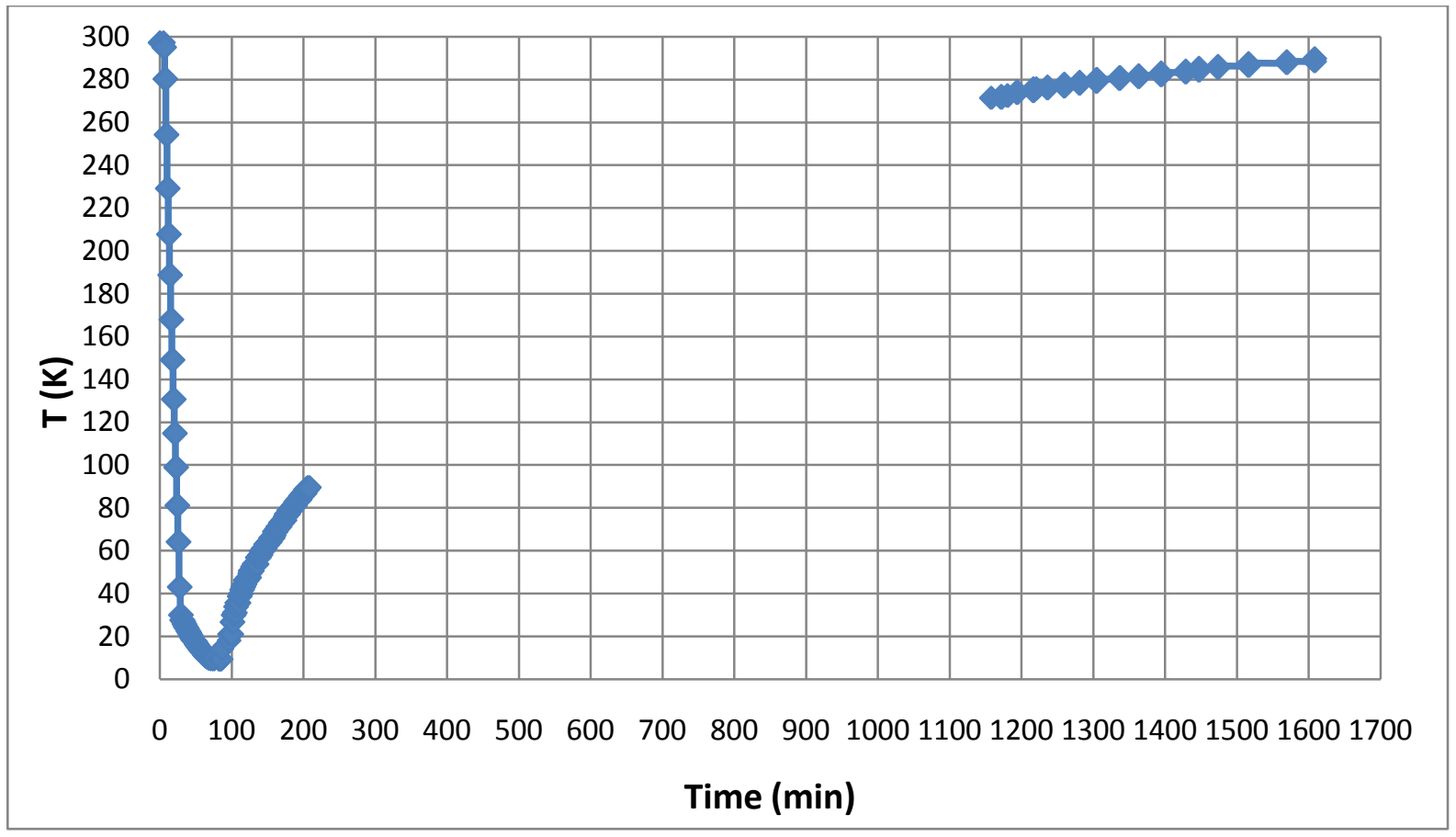

Figure 4.27: The change of temperature by cooling down and warming up as a function of the time.

For cooling down the sample by using the cold head approximately a time of 80 minutes is necessary till a temperature of $4.7 \mathrm{~K}$ can be reached. After stopping the cooling process the sample starts to warm up. Without using any heater, it takes app. 27 hours till the sample is again at room temperature (Figure 4.27). With the help of the heater, it is possible to warm up the cold sample faster.

The Figure 4.28 shows the change of the temperature depending on the power of the heater, while the cooling is still on.

In Figure 4.29 the spectra of the residual gas in the system at room temperature and at $8 \mathrm{~K}$ are compared. It is to be seen that the amount of $\mathrm{H}_{2} \mathrm{O}, \mathrm{CO}, \mathrm{CO}_{2}$ and $\mathrm{CH}_{4}$ decreases due to the cryopumping of the cold sample. Also the amount of the $\mathrm{H}_{2}$ decreases as a fraction of $\mathrm{H}_{2} \mathrm{O}$. 
Chapter 4- Design of the system for SEY-Measurements at cryogenic temperatures

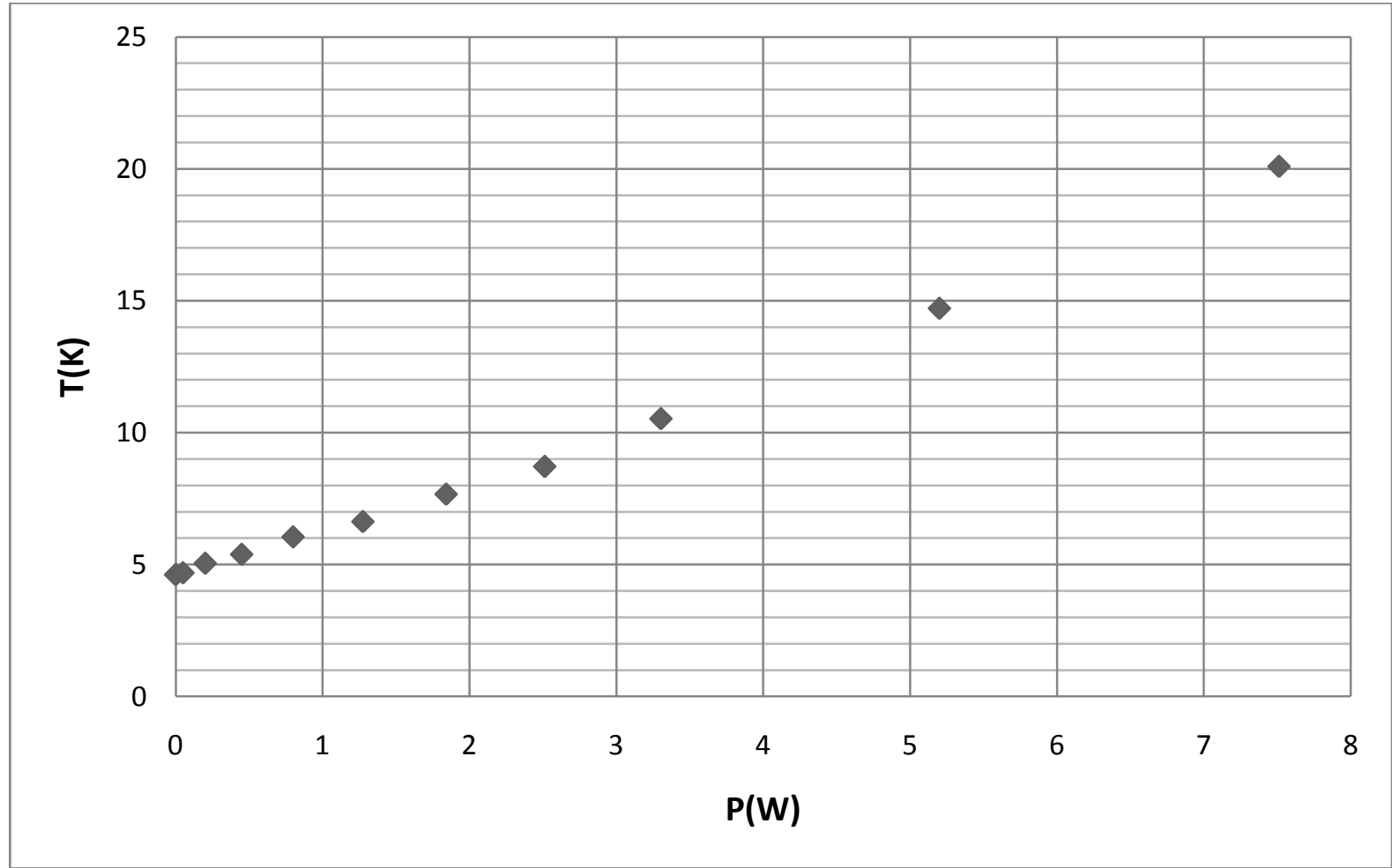

Figure 4.28: Heat load on the cold sample.

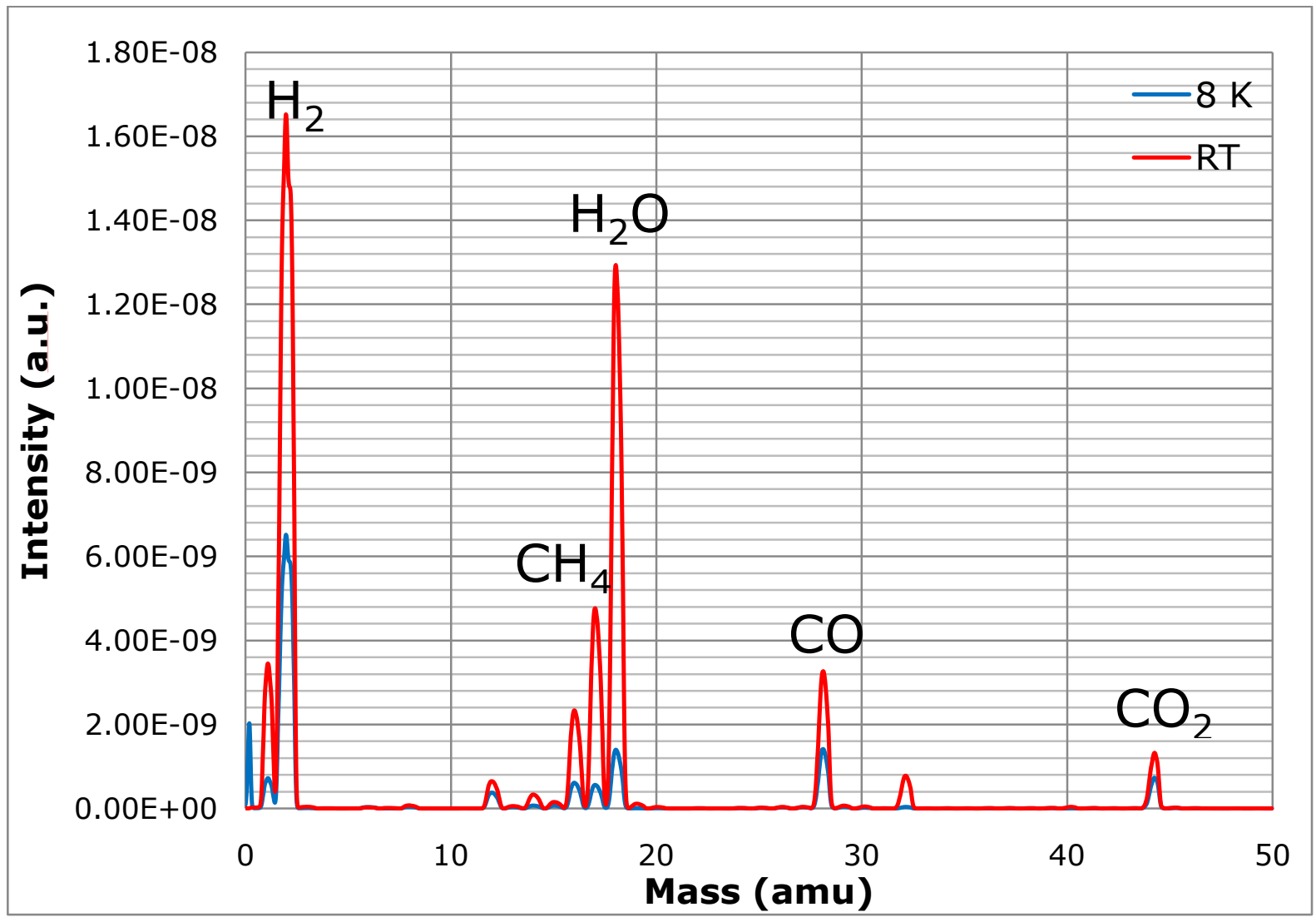

Figure 4.29: Spectrum of the residual gas of the baked vacuum chamber at room temperature and at $8 K$. 


\section{Chapter 4- Design of the system for SEY-Measurements at cryogenic temperatures}

\subsection{Work function and the Kelvin Probe}

The work function (WF) is the least amount of energy required to remove an electron from the surface of a conducting material, to a point just outside the metal with zero kinetic energy. As the electron has to move through the surface region, its energy is influenced by the chemical, electric and mechanical characteristics of the region. Hence the WF is an extremely sensitive indicator of surface condition and is affected by absorbed, adsorbed or evaporated layers, surface reconstructions, surface charging, oxide layer imperfections, surface and bulk contamination, etc.

The Kelvin Probe is a non-contact, non-destructive vibrating capacitor device used to measure the work function difference, or for non-metals, the surface potential, between a conducting specimen and a vibrating tip.

When two materials with different work functions are brought together, electrons in the material with the higher work function flow to the one with the lower work function. If these materials are made into a parallel plate capacitor, equal and opposite surface charges form. The voltage developed over this capacitor is called the contact potential and measuring it is done by applying an external backing potential to the capacitor until the surface charges disappear, at that point the backing potential will equal the contact potential.

The traditional Kelvin Probe was first postulated by the renowned Scottish scientist W.Thomson, later Lord Kelvin, in 1861. It consists of a flat circular electrode (termed the reference electrode) suspended above and parallel to a stationary electrode (the specimen), thus creating a simple capacitor. In 1932 William Zisman introduced a new method to measure the contact potential. He mounted a vibrating reference surface, or tip, just above a sample electrode. The output voltage varies periodically as the tip vibrates, and the peak-topeak voltage depends on the difference between the contact potential and backing voltage.

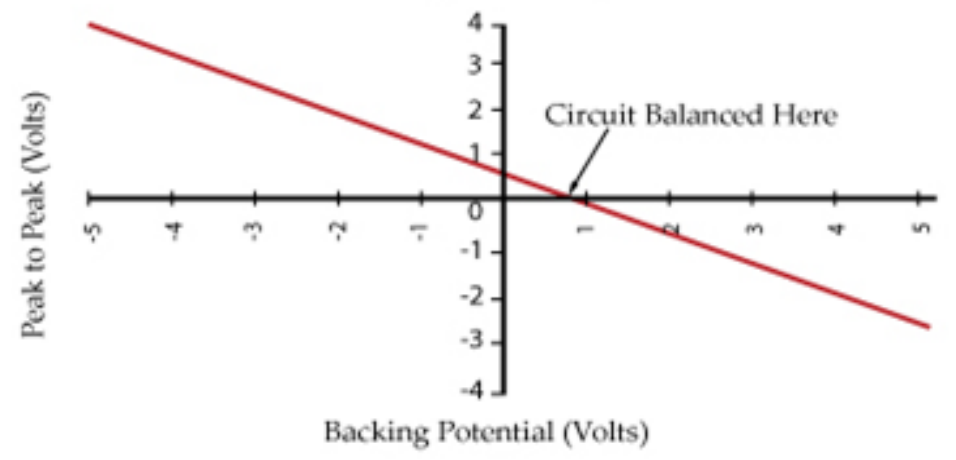

When $\mathrm{V}_{\mathrm{ptp}}=0$ the Work Function or Contact Potential of the surface is equal and opposite to $\mathrm{Vb}$. 


\section{Chapter 4- Design of the system for SEY-Measurements at}

\section{cryogenic temperatures}

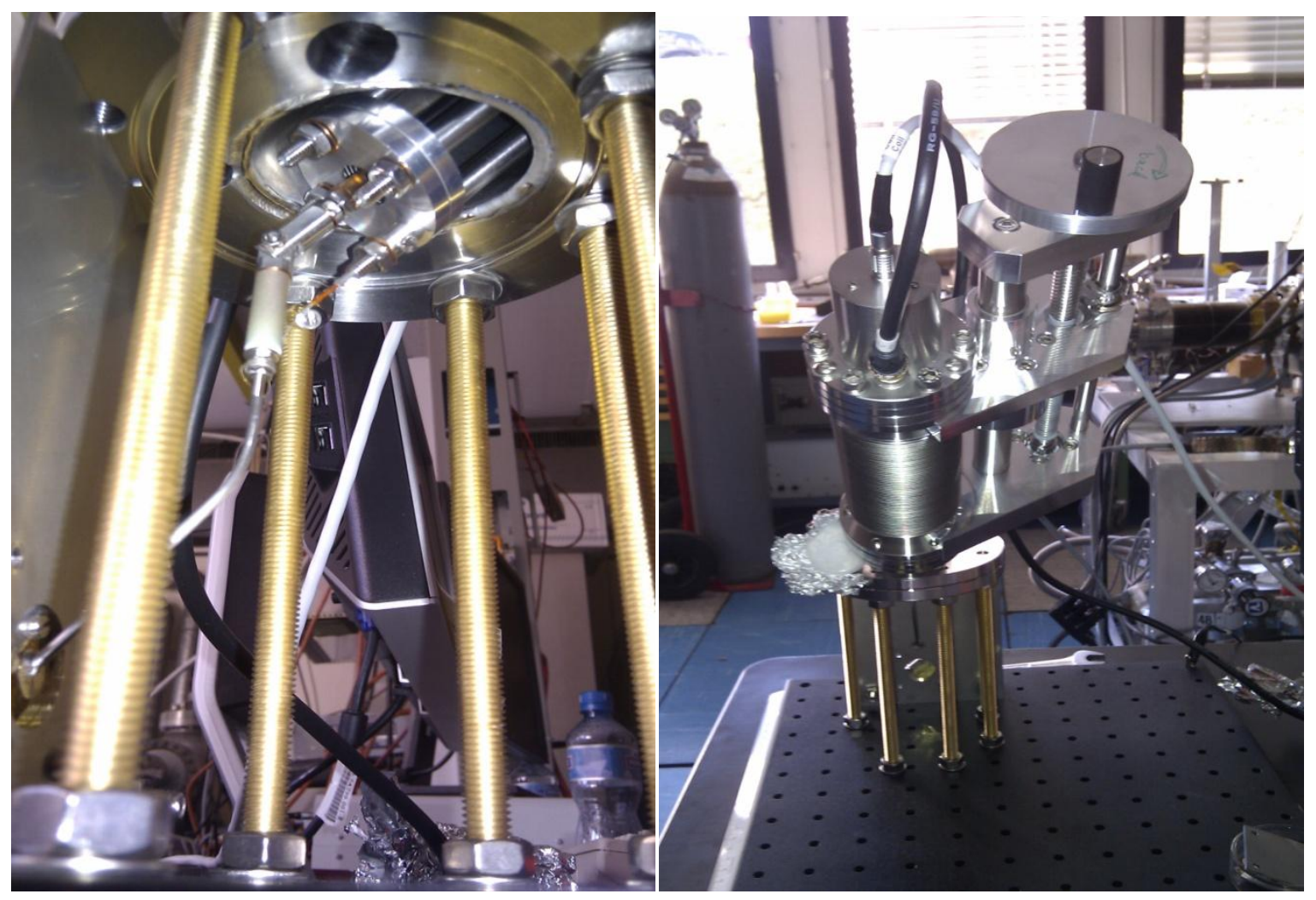

Figure 4.30: Photos of the Kelvin Probe taken before assembling on the vacuum system.

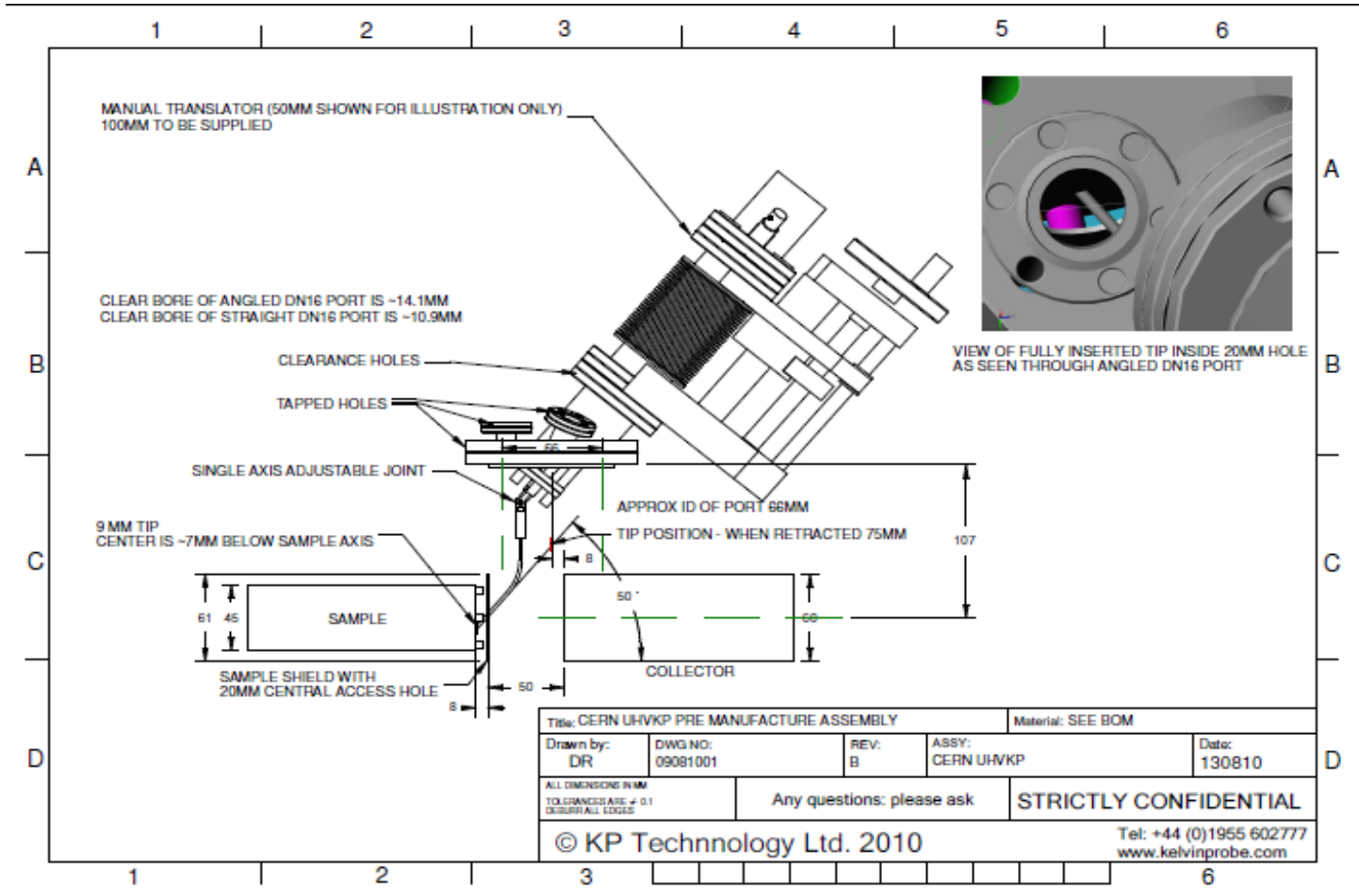

Figure 4.31: Drawing of the Kelvin Probe. It is mounted on a CF63 flange. It has a work function resolution of $1-3 m e V$. The vibrating tip is made of stainless steel. 


\section{Secondary electron emission from cold surfaces as a function of the gas adsorption}

\subsection{Reference measurements with an existing system at room temperature}

In order to be able to verify the results of the newly built system, measurements with an already existing system have been done, which was built to perform SEY measurements at room temperature. The system is described in [30]. In that way acquired results have been compared with the results of the new system.

\subsubsection{SEY measurements with different samples}

Four different samples - Aluminium, copper, stainless steel and a gold film placed on a copper sample- were mounted on a rotating sample holder (Figure 5.1). The samples used for the measurements are chemically cleaned and they are air exposed. The energy range of the primary electrons varies from $100 \mathrm{eV}$ to $3000 \mathrm{eV}$. The results of these measurements are shown below in figure 5.2. The vacuum system used for the measurements is not baked, and the pressure was $6 \cdot 10^{-8} \mathrm{mbar}$. 


\section{Chapter 5-Results}

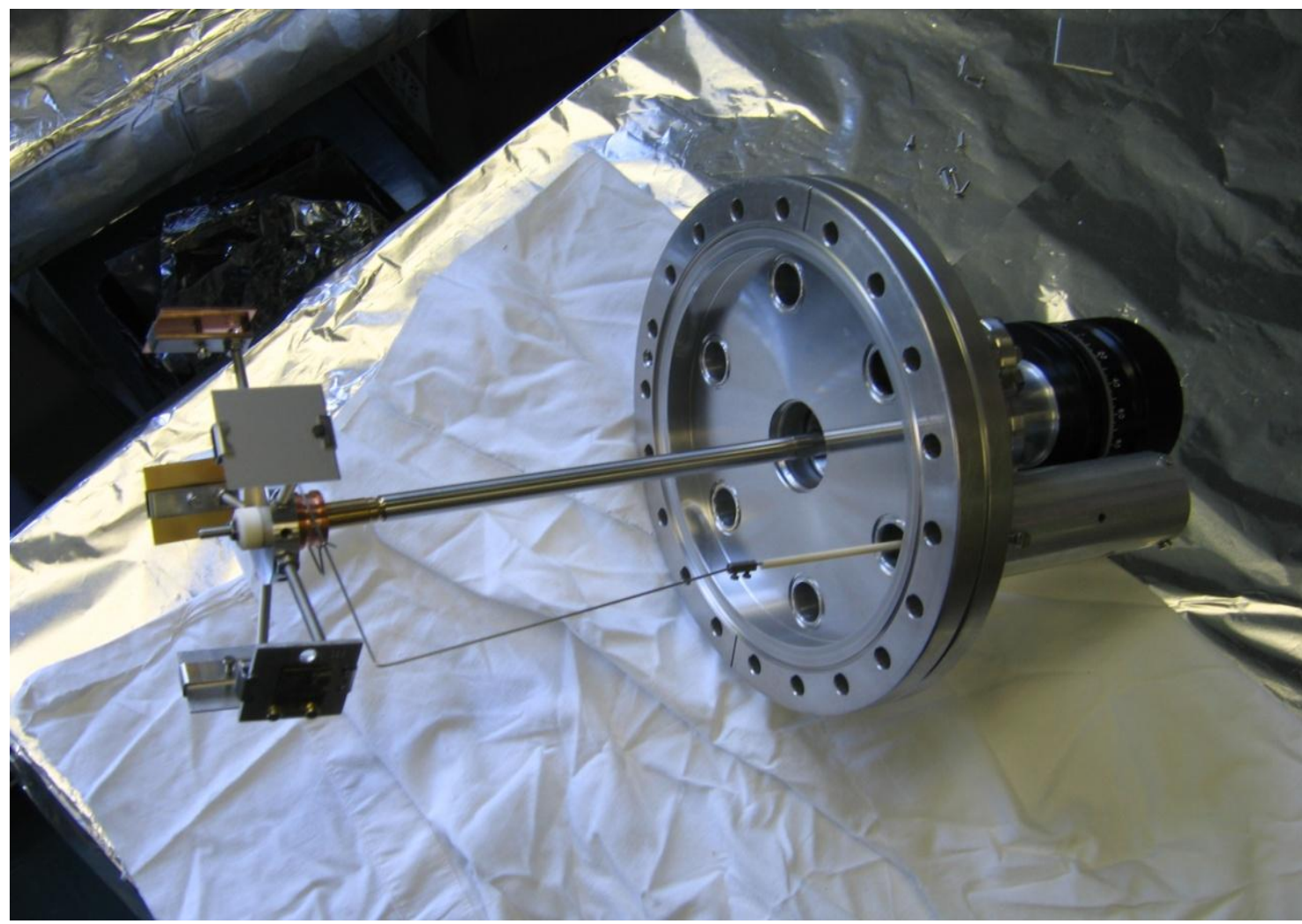

Figure 5.1: The rotating sample holder with mounted samples.

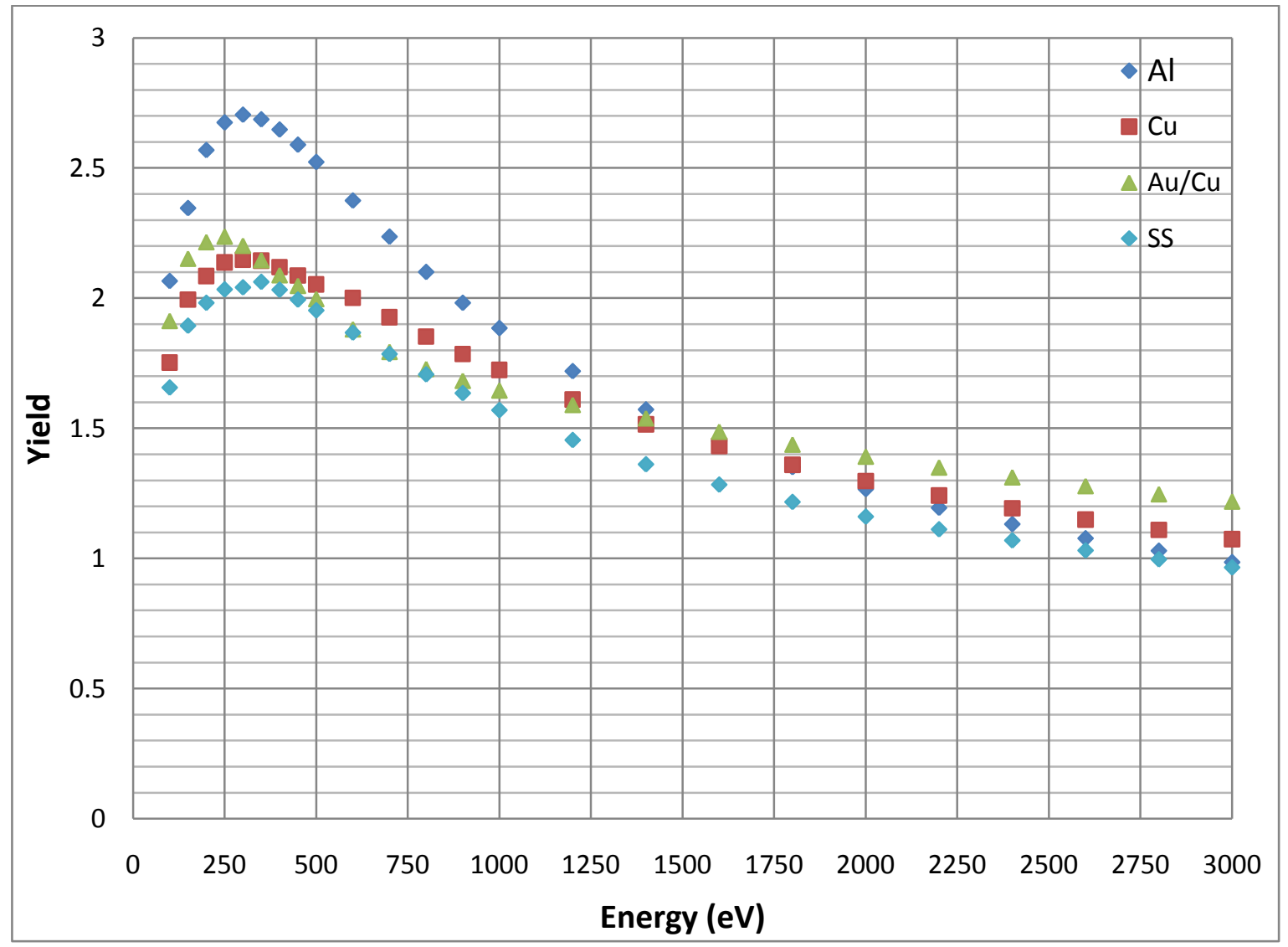

Figure 5.2: SEY results for different samples at room temperature in a non-baked system. 
The yield is changing for different materials. Aluminium has the maximum yield of 2.7 at an energy of $300 \mathrm{eV}$, whereas copper has a maximum yield of 2.1. The values of the maximum yield for SS and Au on Cu are 2.06 and 2.23 , respectively.

\subsubsection{Investigations of the effect of microstructures}

As explained in chapter 2 the microstructure is a factor which can change the SEY. In order to investigate this effect, a drilled copper sample has been manufactured with $92 \mathrm{holes} / \mathrm{cm}^{2}$ with a hole diameter of $1 \mathrm{~mm}$ (Figure 5.3).
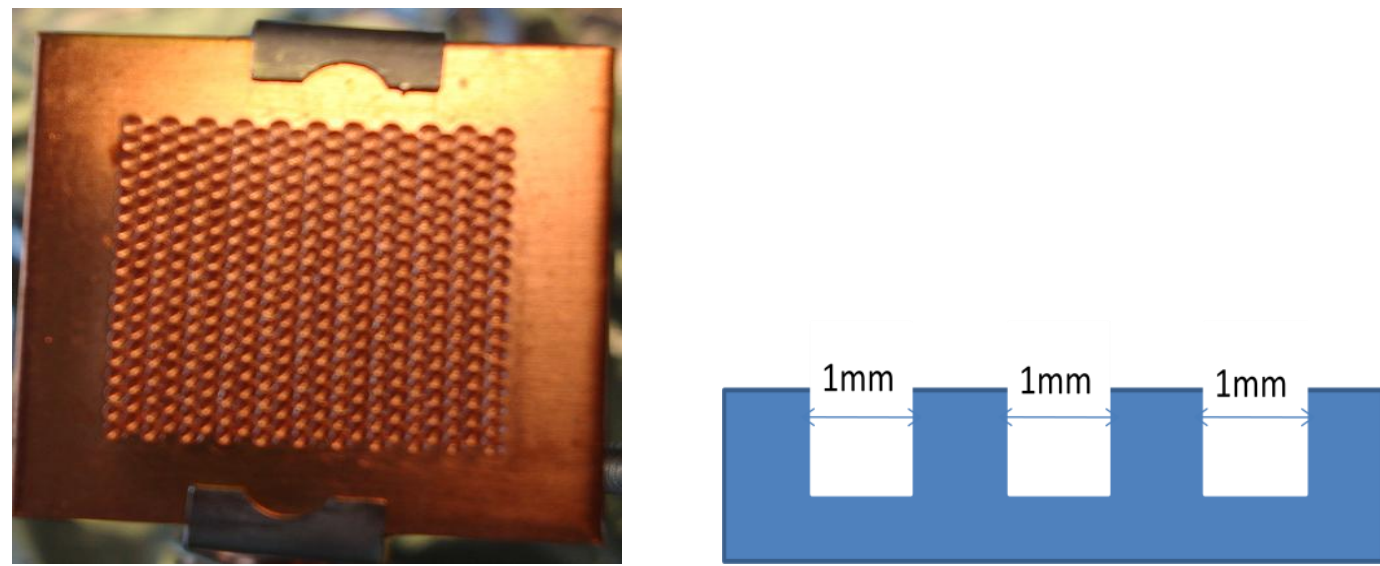

Figure 5.3: Drilled copper sample with 92 holes $/ \mathrm{cm}^{2}$ each with $1 \mathrm{~mm}$ diameter.

The comparison of the drilled copper and air exposed copper without microstructures are shown in Figure 5.4.

\section{Drilled Cu vs. air exposed $\mathrm{Cu}$}

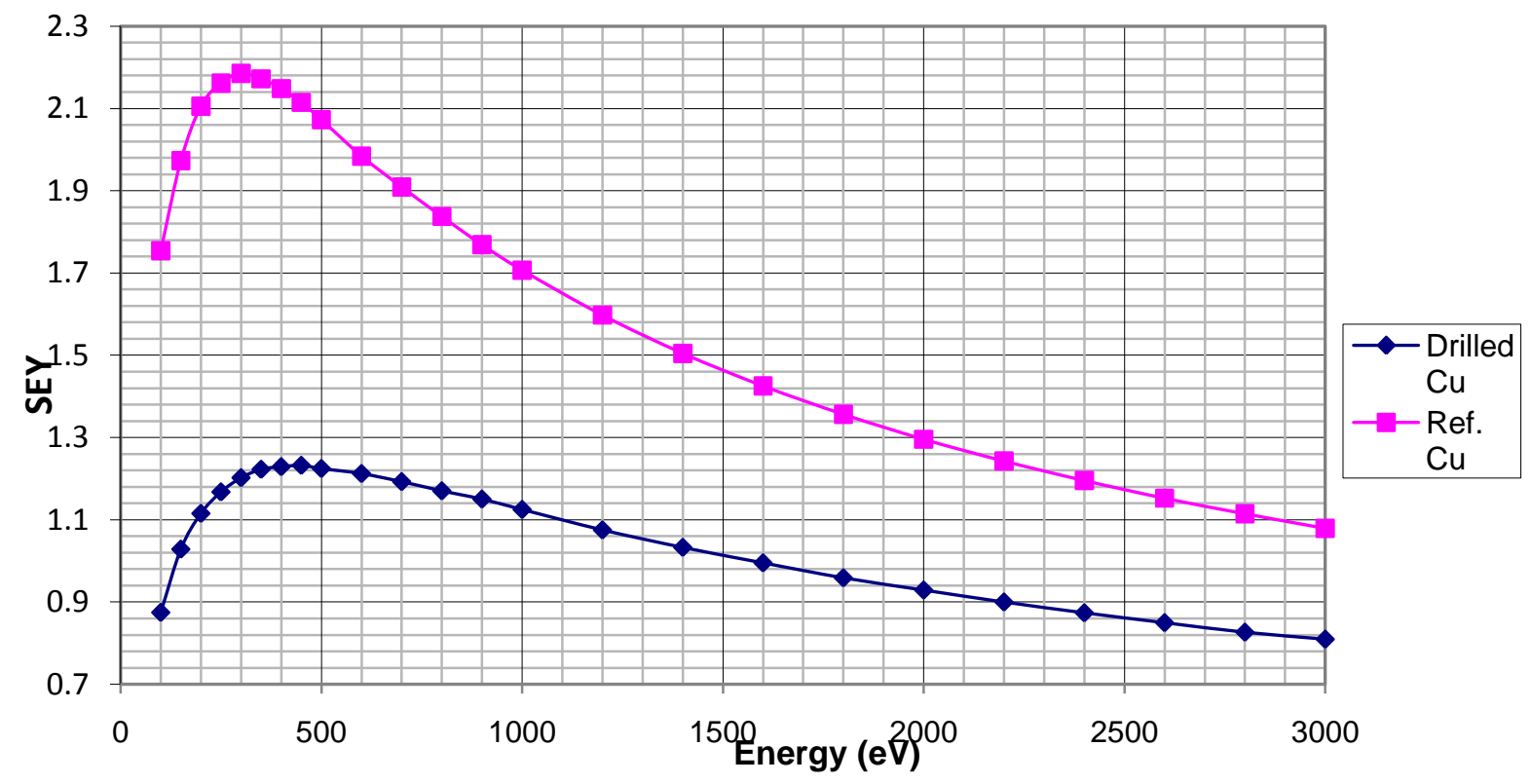

Figure 5.4: Yield of the drilled copper compared with the non drilled air exposed copper. 


\subsection{Description of the SEY experiment at cryogenic temperatures}

The aim of this work is a quantitative and qualitative investigation of secondary electron emission on cold surfaces as a function of gas coverage. In chapter 2 it has been already shown that the secondary electrons produced in the vacuum system have a major influence on the performance of the accelerator.

The idea is to measure the SEY of a sample, which is kept at $4.7 \mathrm{~K}$ as a function of physisorbed gases; in this case $\mathrm{N}_{2}, \mathrm{Kr}, \mathrm{CO}, \mathrm{CO}_{2}, \mathrm{C}_{2} \mathrm{H}_{6}$ and $\mathrm{CH}_{4}$. SEY of adsorbed $\mathrm{CO}, \mathrm{CO}_{2}$ and $\mathrm{CH}_{4}$ have been measured because they are the most common gases in the CERN- LHC accelerator and in most other Ultra High Vacuum Systems. Measurements have been performed with $\mathrm{N}_{2}$, because of its importance as calibration gas for most of the pressure gauges and pumps. Also measurements with $\mathrm{Kr}$ have been done, to compare the results with existing works. Measurements with $\mathrm{C}_{2} \mathrm{H}_{6}$ are performed to compare the results with $\mathrm{CH}_{4}$ in order to find common points. The measurements of the secondary electron yield are needed to predict the vacuum behaviour in the LHC and to understand the mechanism of SEY from physisorbed gas layers.

Copper was used as the first sample, it is the material used for the beam screen; afterwards measurements have been performed with Aluminium, electro polished copper, Niobium and Carbon. Different gas layers were physisorbed on the cooled sample and the yield was measured for different amounts of gas layers.

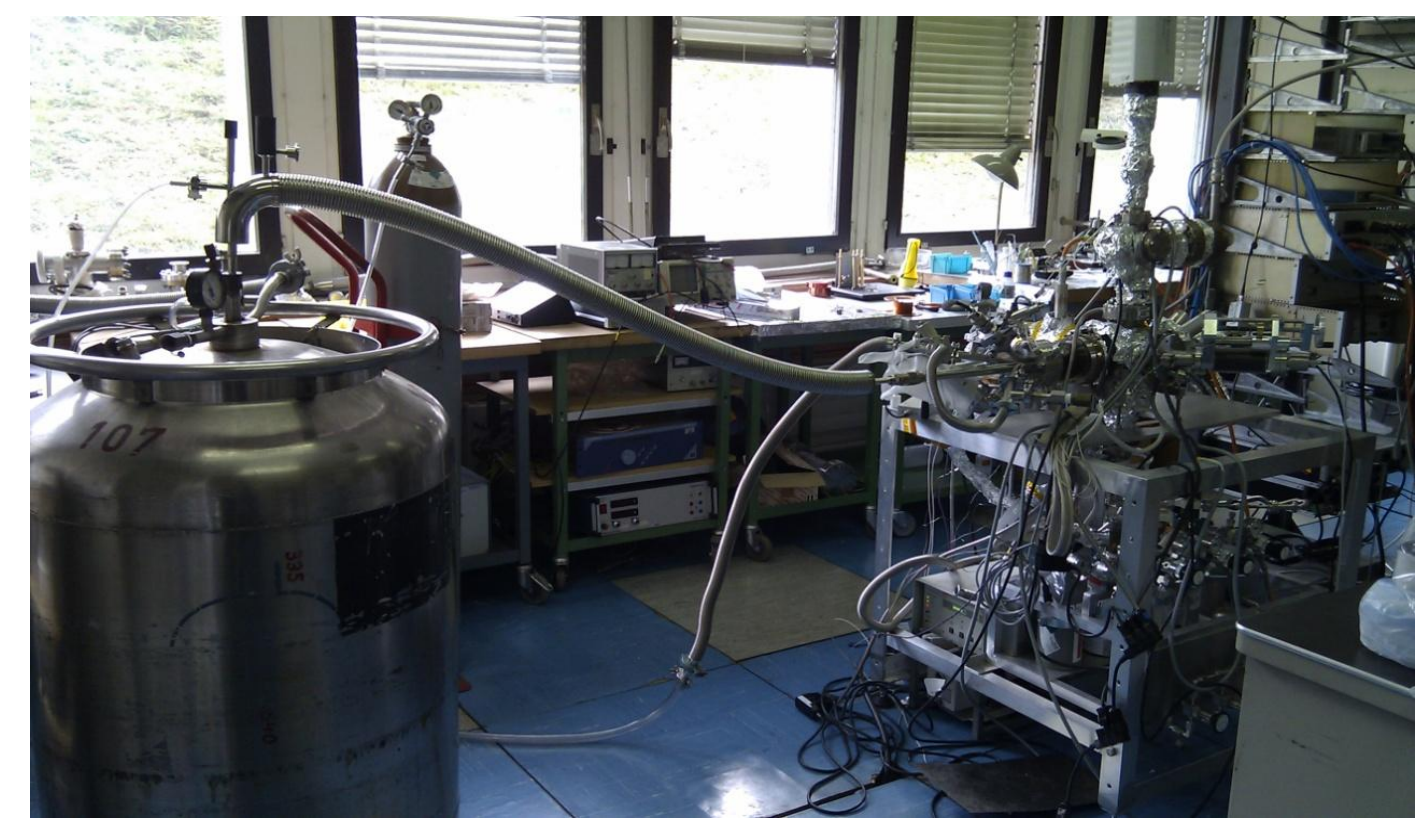

Figure 5.5: SEY measurements system connected to the helium Dewar for cooling down. 


\subsection{Results}

\subsubsection{SEY of copper}

Before starting the measurements as a function of gas coverage the yield of copper at $4.7 \mathrm{~K}$ has been measured. All the measurements are reproducible. The measured yield is decreasing with decreasing temperature (Figure 5.6). The reason for this decrease could be the adsorption of the residual gases in the vacuum system on the copper sample due to the cooling. The number of monolayers of adsorbed gas during cooling down is about 0.3 by a pressure of $1.5 \cdot 10^{-9} \mathrm{mbar}$.

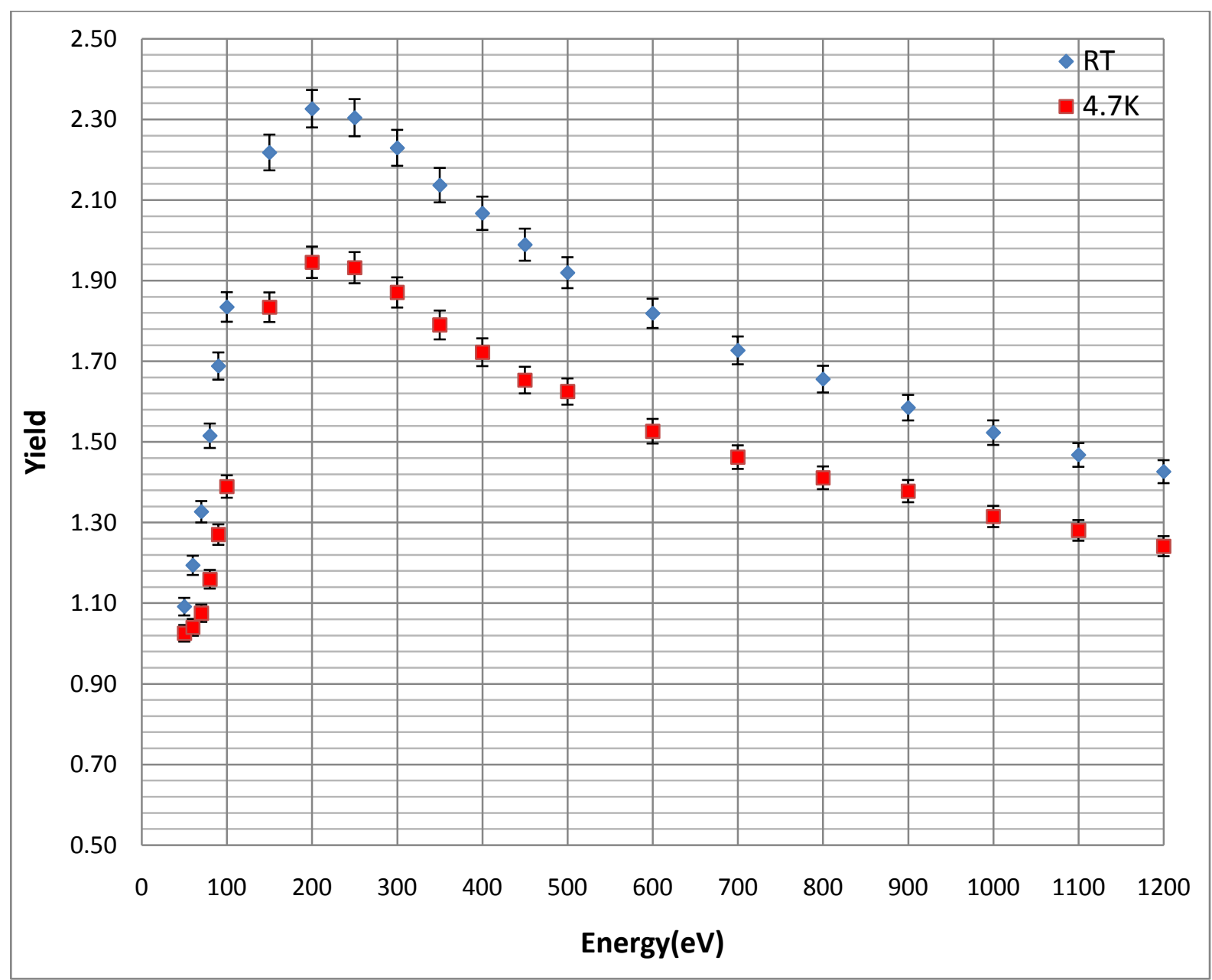

Figure 5.6: SEY of copper at different temperatures. The yield is decreasing with decreasing temperature. 


\subsubsection{SEY of copper as a function of $\mathrm{Kr}, \mathrm{N}_{2}, \mathrm{CO}_{2}, \mathrm{CO}, \mathrm{CH}_{4}$ and $\mathrm{C}_{2} \mathrm{H}_{6}$ adsorption}

The variation of the SEY as a function of 10 monolayers $\mathrm{Kr}, \mathrm{N}_{2}, \mathrm{CO}_{2}, \mathrm{CO}, \mathrm{CH}_{4}$ and $\mathrm{C}_{2} \mathrm{H}_{6}$ physisorbed on the copper is shown in figure 5.7 .

For each measurement it was essential to have new, fresh layers of adsorbed gases in order to avoid charging. Therefore after each measurement the previous gas layers have been removed by warming up the sample and new layers have been adsorbed for the following measurement. This process was repeated for all the gases and all the samples.

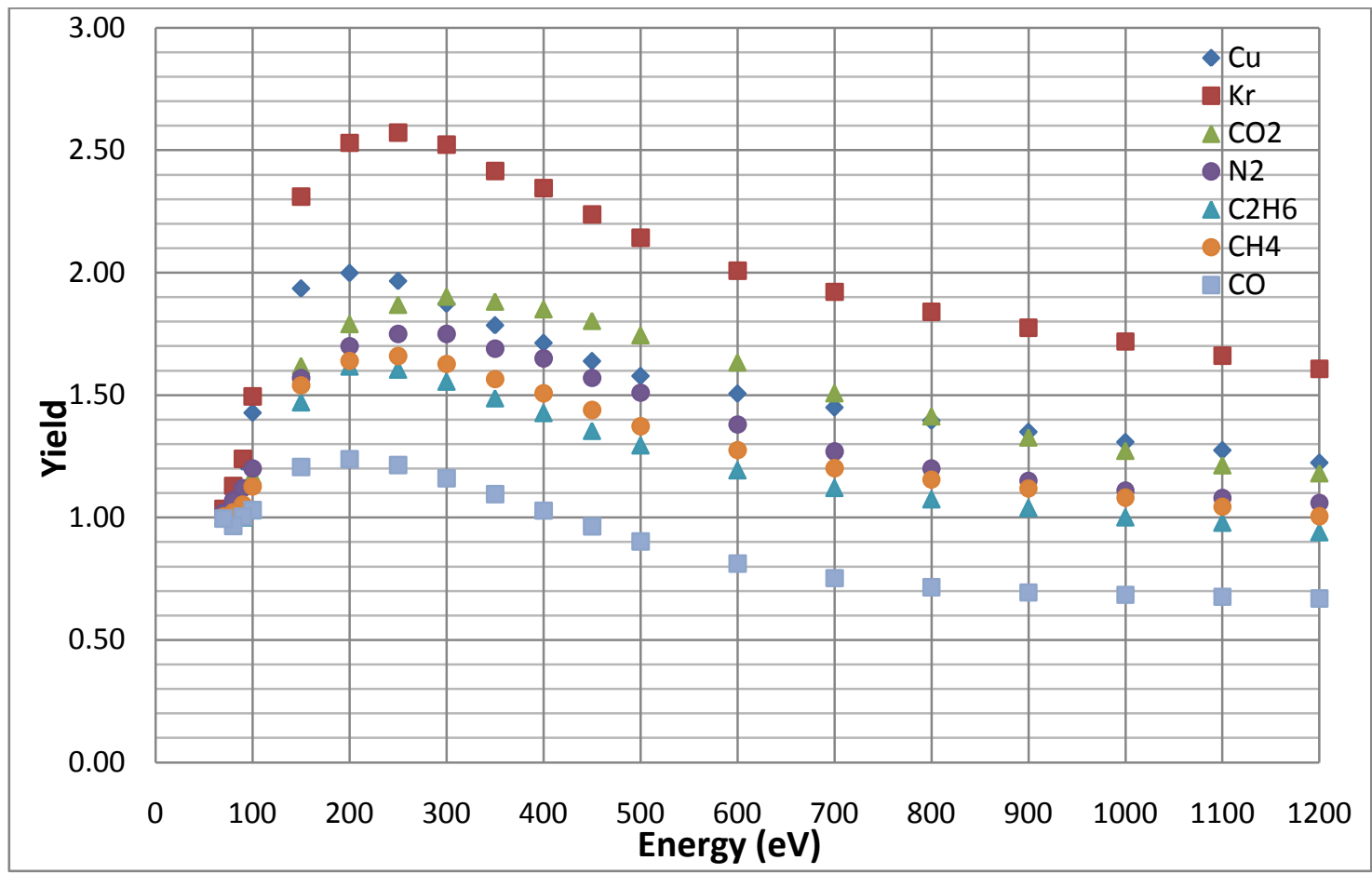

Figure 5.7: The secondary electron yield of 10 monolayers of $\mathrm{Kr}, \mathrm{N}_{2}, \mathrm{CO}_{2}, \mathrm{CO}, \mathrm{CH}_{4}$ and $\mathrm{C}_{2} \mathrm{H}_{6}$ physisorbed on copper.

The adsorption of 10 monolayers Krypton increases the yield of copper substrate, while the adsorption of each 10 monolayers of $\mathrm{N}_{2}, \mathrm{CO}_{2}, \mathrm{CO}, \mathrm{CH}_{4}$ and $\mathrm{C}_{2} \mathrm{H}_{6}$ decreases the yield.

The energy for maximum yield is different for different gases. While $\mathrm{Kr}, \mathrm{CH}_{4}$ and $\mathrm{N}_{2}$ have the maximum yield at $250 \mathrm{eV} ; \mathrm{CO}$ and $\mathrm{C}_{2} \mathrm{H}_{6}$ have it like air exposed $\mathrm{Cu}$ at $200 \mathrm{eV} . \mathrm{CO}_{2}$ has the maximum yield at $300 \mathrm{eV}$. The secondary electron yield for each gas with different numbers of monolayers as a function of the primary electron energy is shown in figures 5.8 till 5.18. For each gas the first series of curves shows the SEY for high coverage and the second series shows the SEY with low coverage. For Krypton there is only one curve with high coverage. For each gas the measurements have been performed 3-4 times and the results are reproducible. The percentage error for the measurements is $3 \%$. 


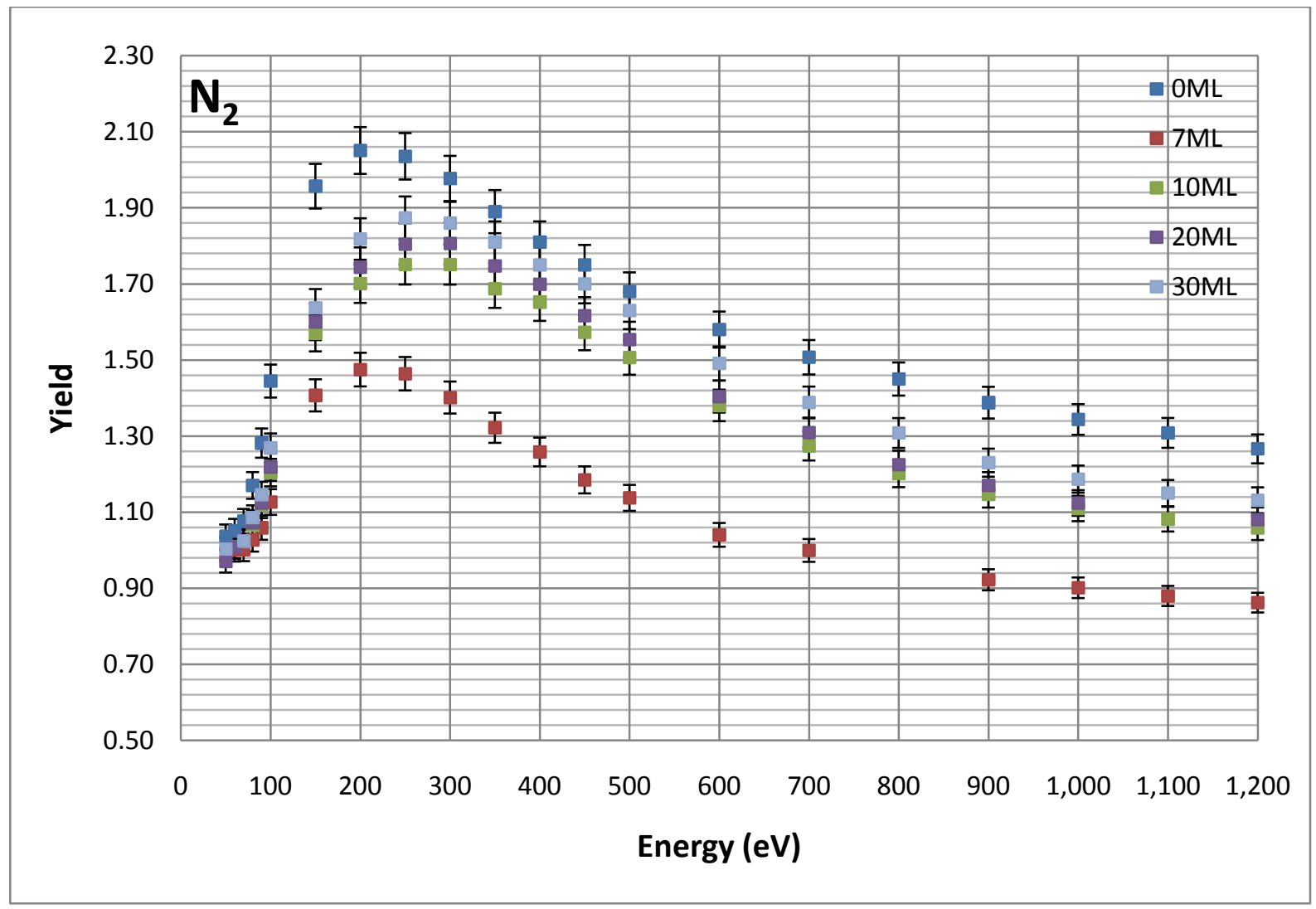

Figure 5.8: SEY of adsorbed $N_{2}$ as a function of energy for different coverages.

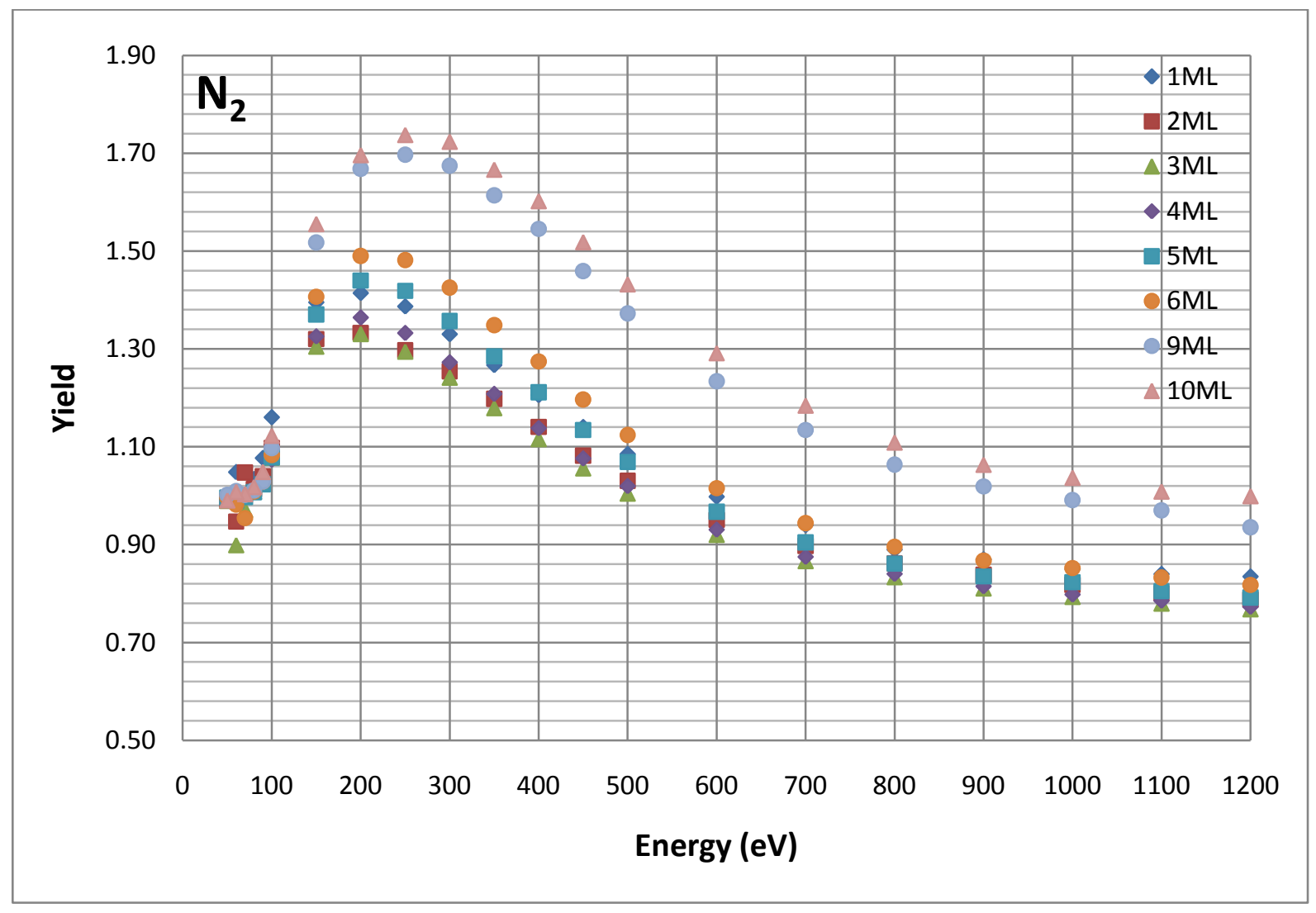

Figure 5.9: SEY of adsorbed $\mathrm{N}_{2}$ as a function of energy for low coverage. 


\section{Chapter 5-Results}

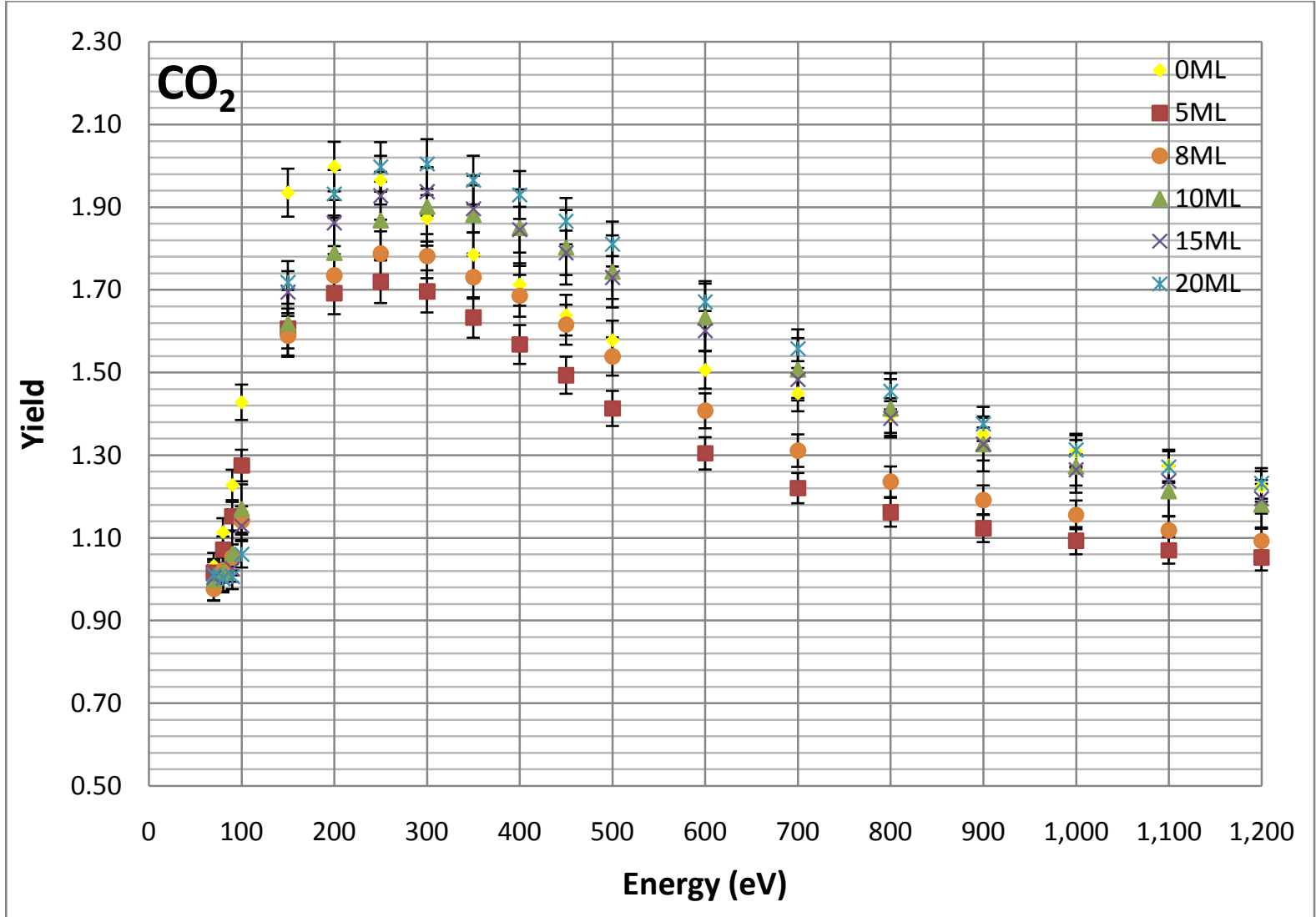

Figure 5.10: SEY of adsorbed $\mathrm{CO}_{2}$ as a function of energy for different coverages.

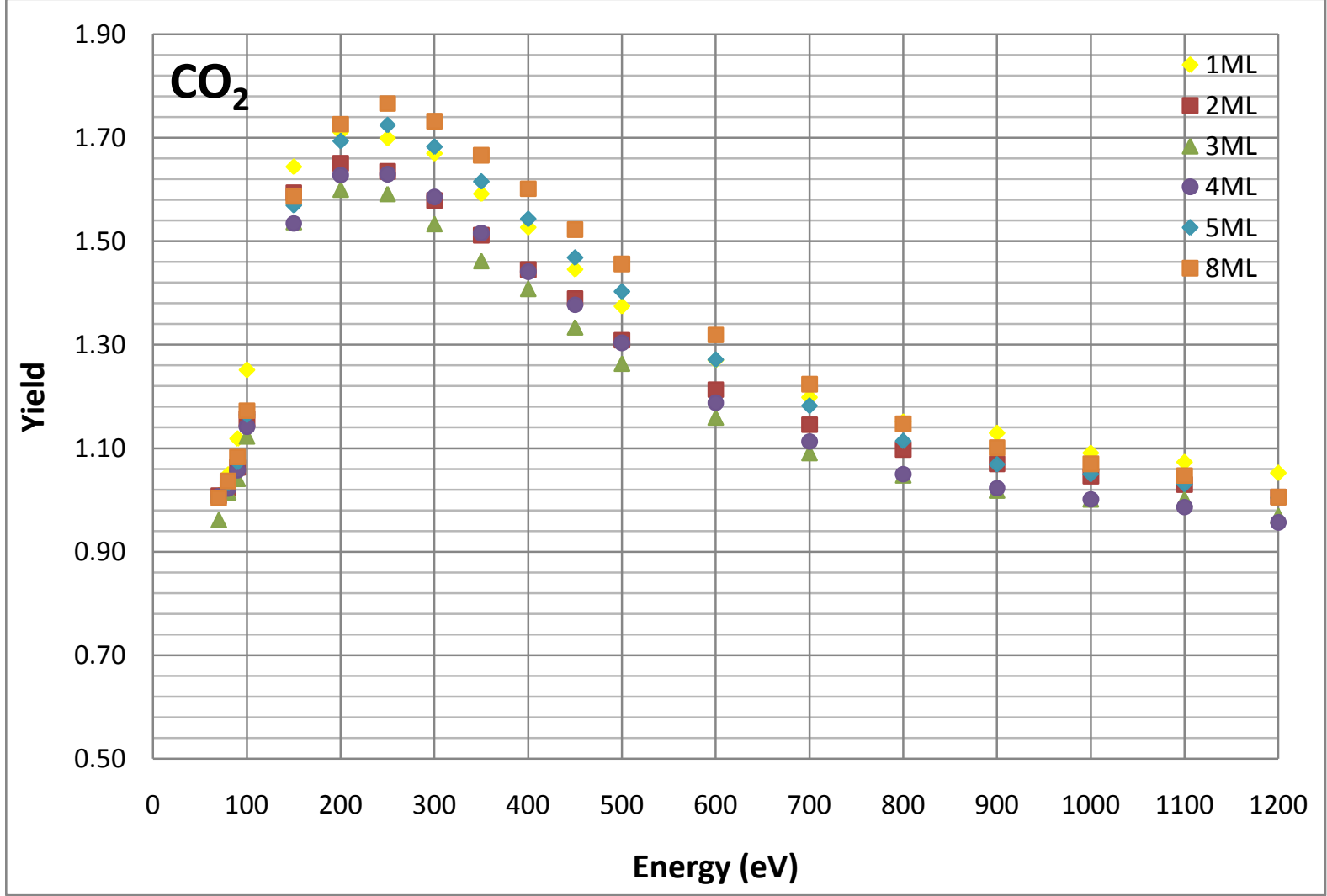

Figure 5.11: SEY of adsorbed CO2 as a function of energy for low coverage. 


\section{Chapter 5-Results}

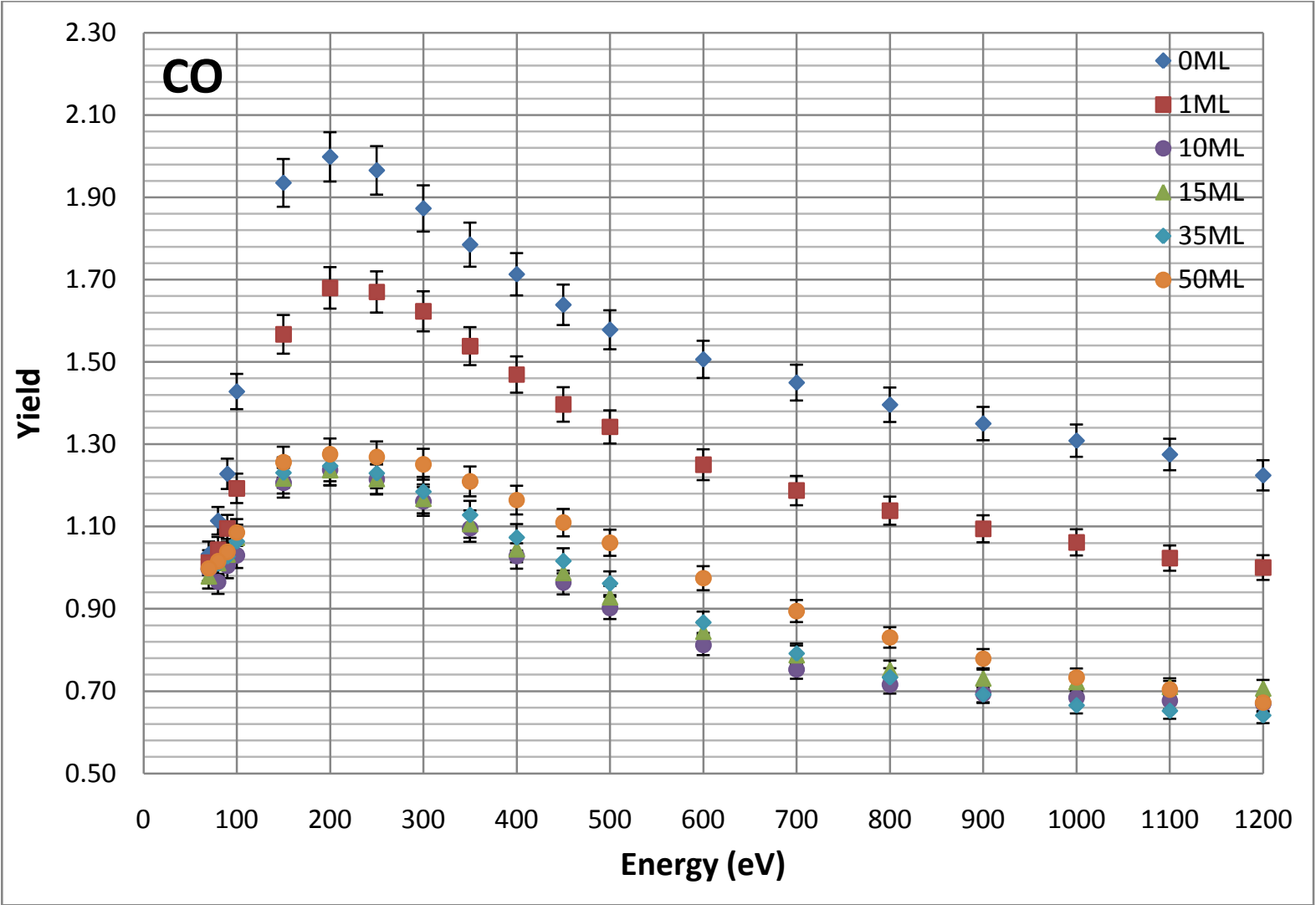

Figure 5.12: SEY of adsorbed CO as a function of energy for different coverages.

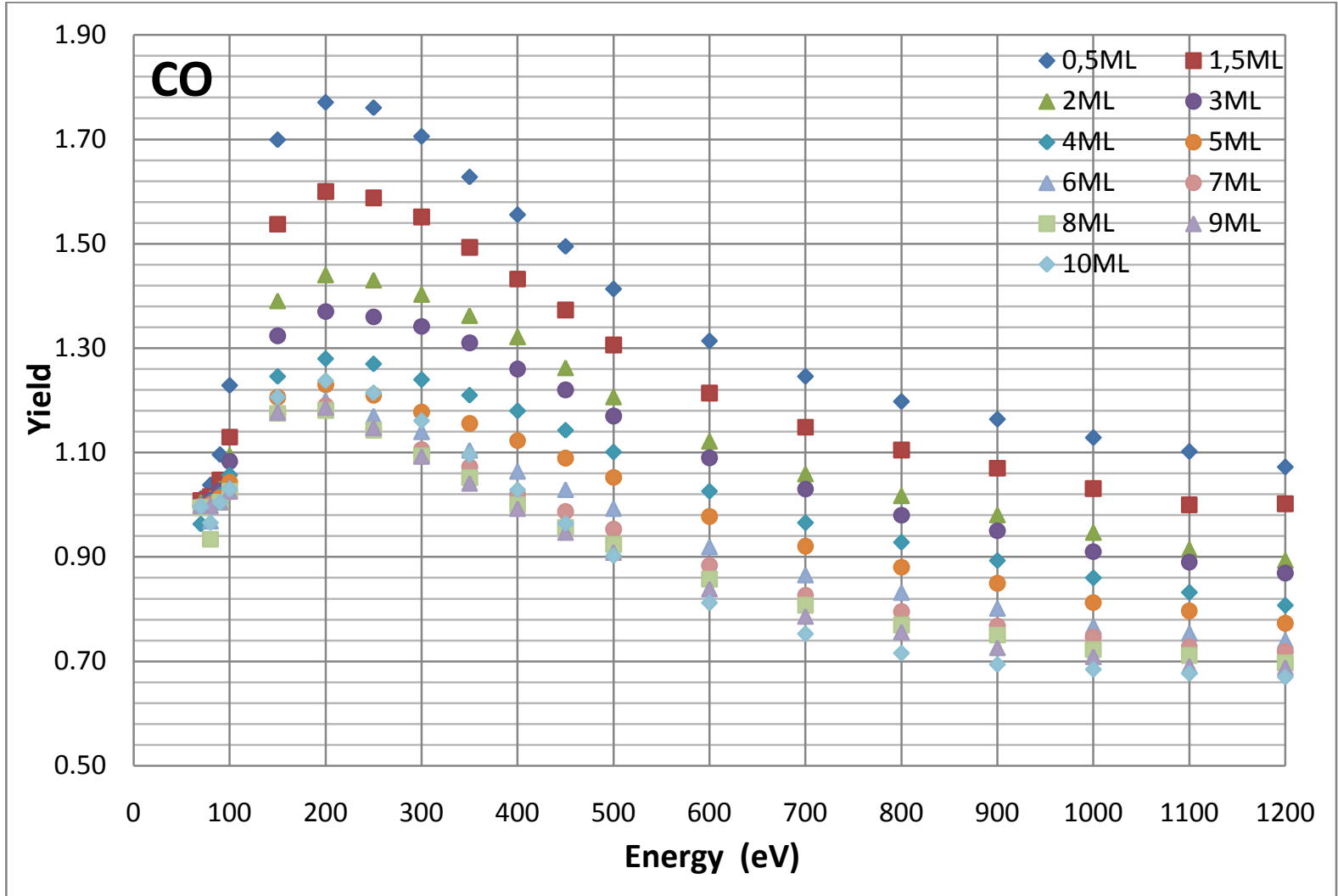

Figure 5.13: SEY of adsorbed CO as a function of energy for low coverage. 


\section{Chapter 5-Results}

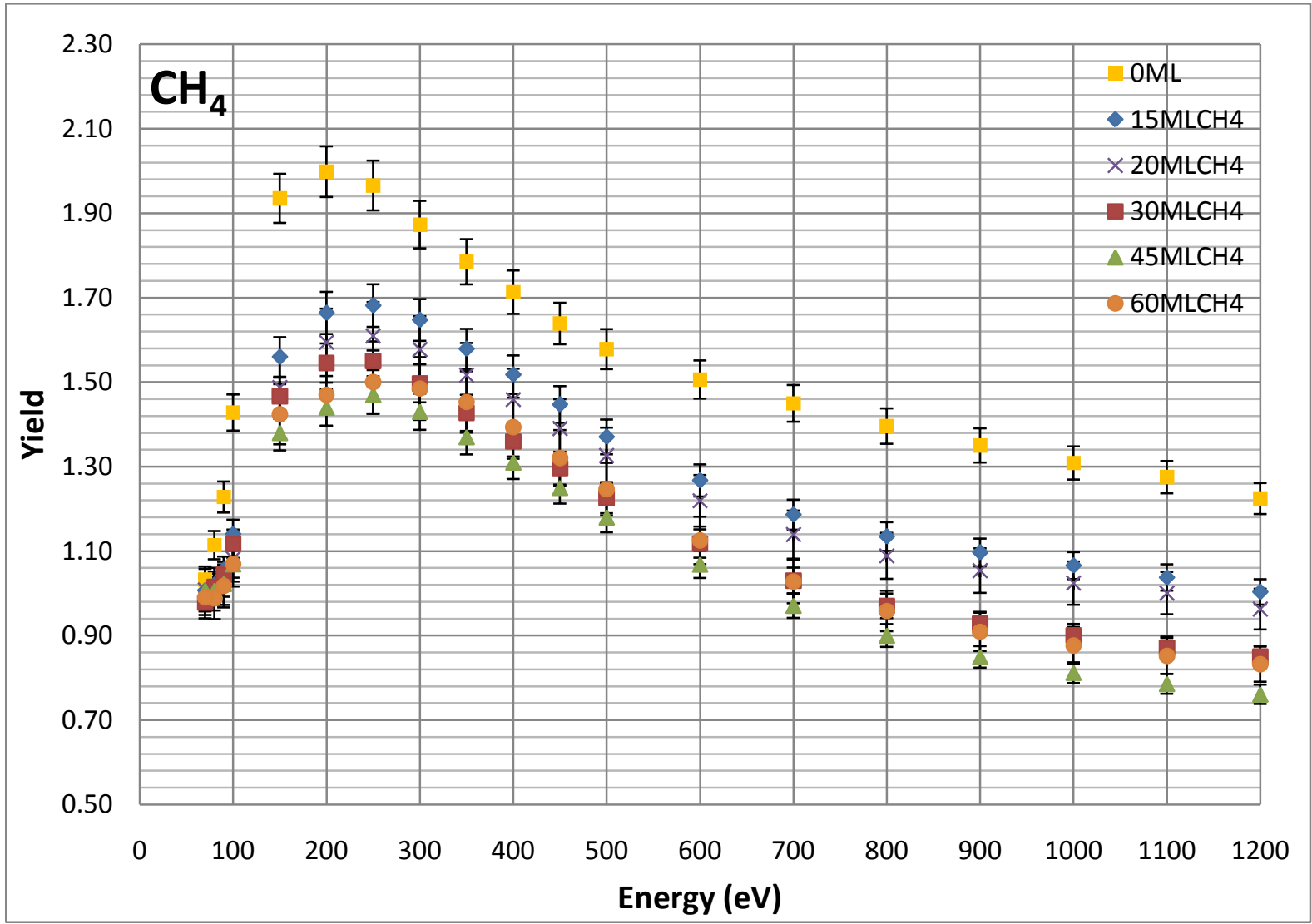

Figure 5.14: SEY of adsorbed $\mathrm{CH}_{4}$ as a function of energy for different coverages.

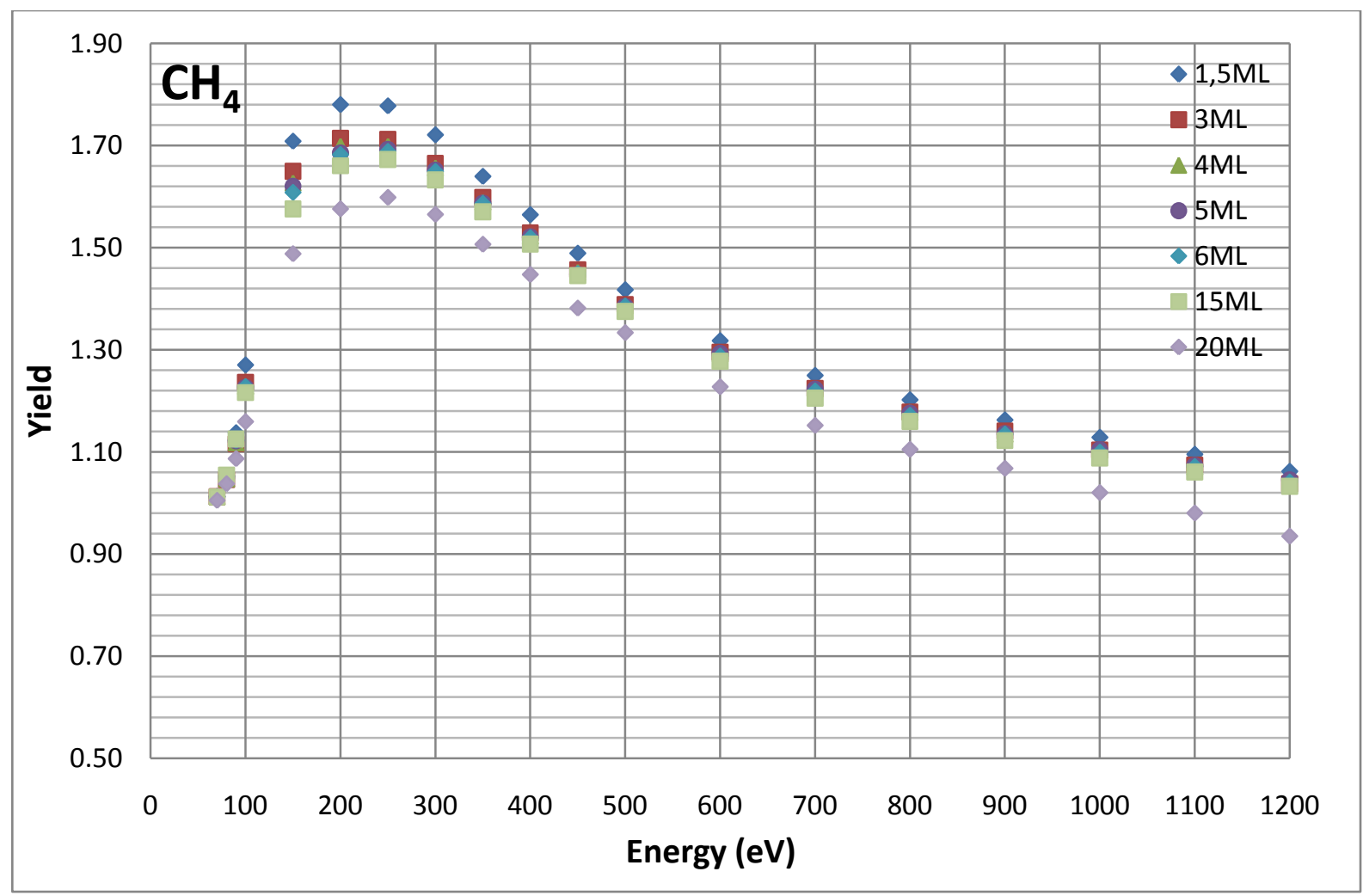

Figure 5.15: SEY of adsorbed $\mathrm{CH}_{4}$ as a function of energy for low coverage. 


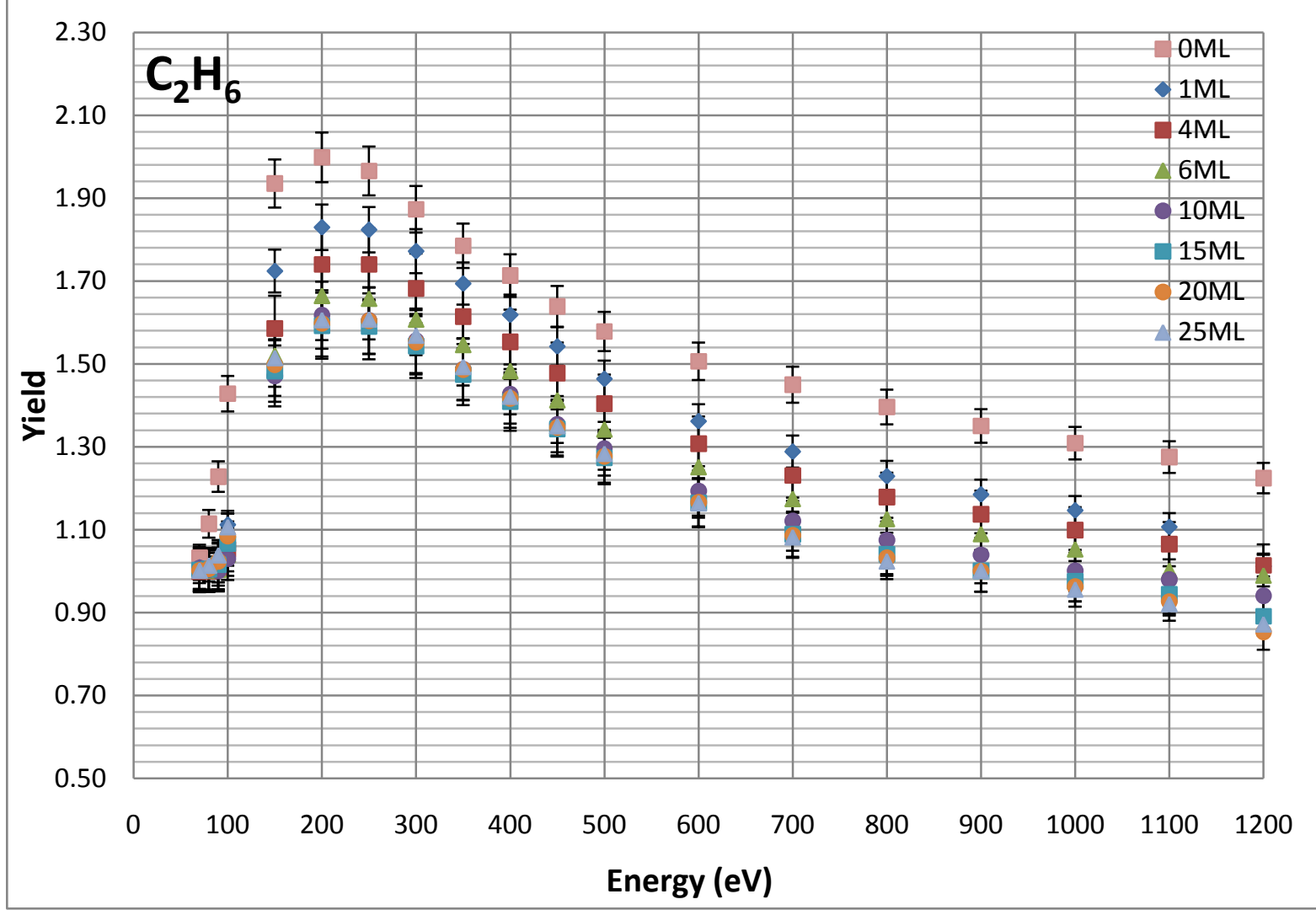

Figure 5.16: SEY of adsorbed $\mathrm{C}_{2} \mathrm{H}_{6}$ as a function of energy for different coverages.

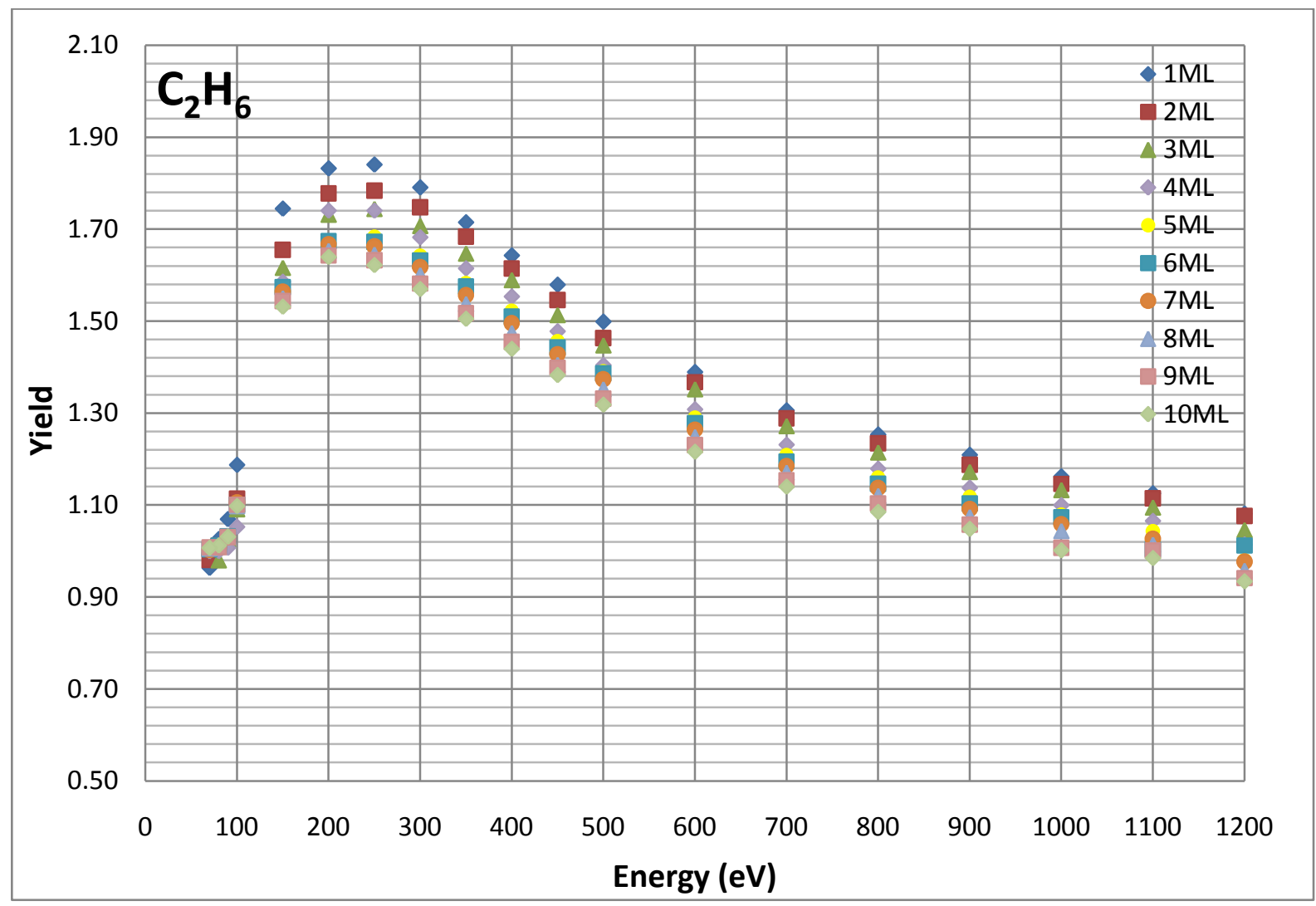

Figure 5.17: SEY of adsorbed $C_{2} H_{6}$ as a function of energy for low coverage. 


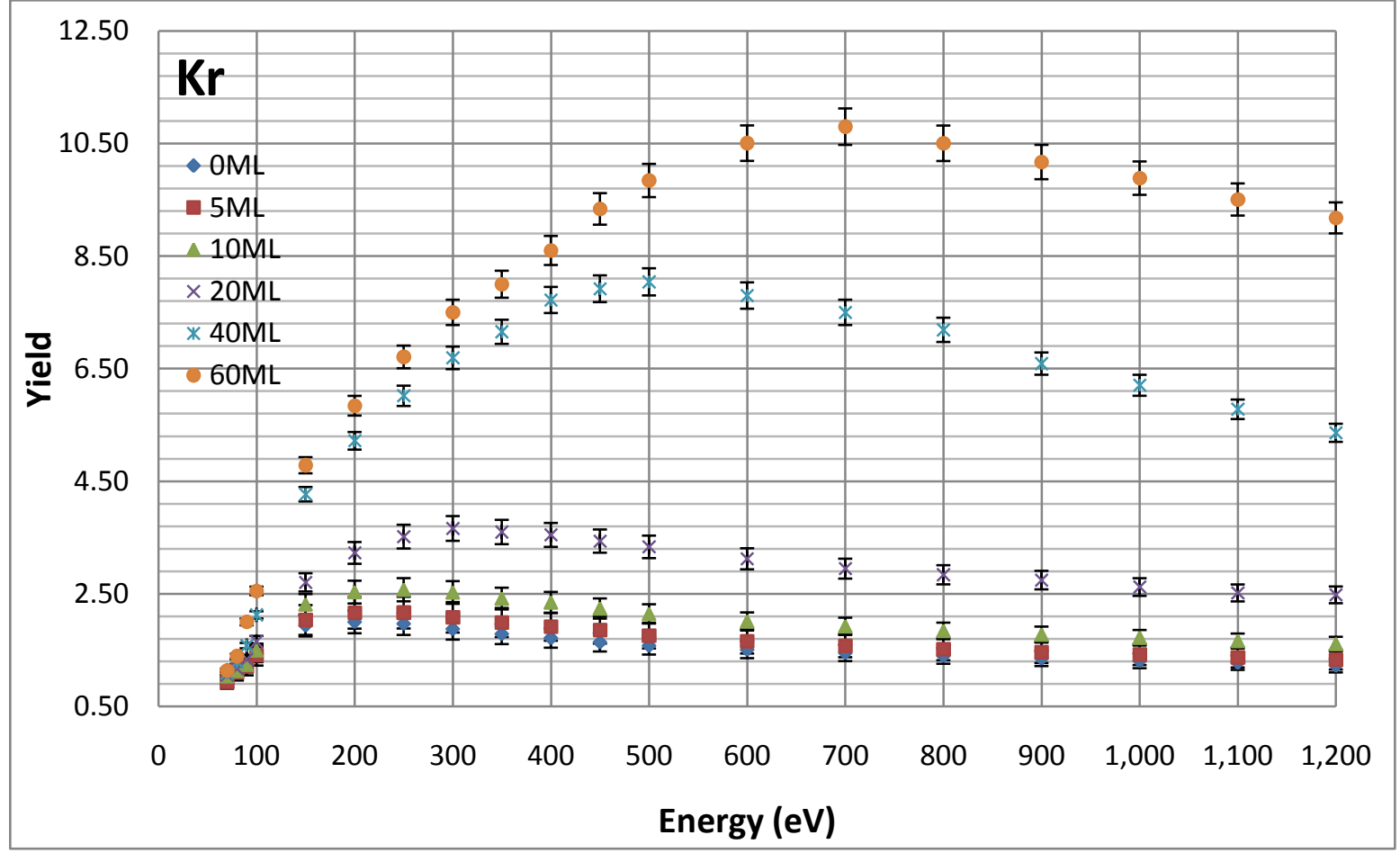

Figure 5.18: SEY of adsorbed Kr as a function of energy for different coverages.

Figures $5.20,5.21,5.22,5.23,5.24$ and 5.25 show the secondary electron yield as a function of the coverage for some selected primary electron energies.

Maximum yield as a function of coverage and the primary energy at the maximum yield as a function of coverage are shown in figure 5.19 and 5.26-5.37.

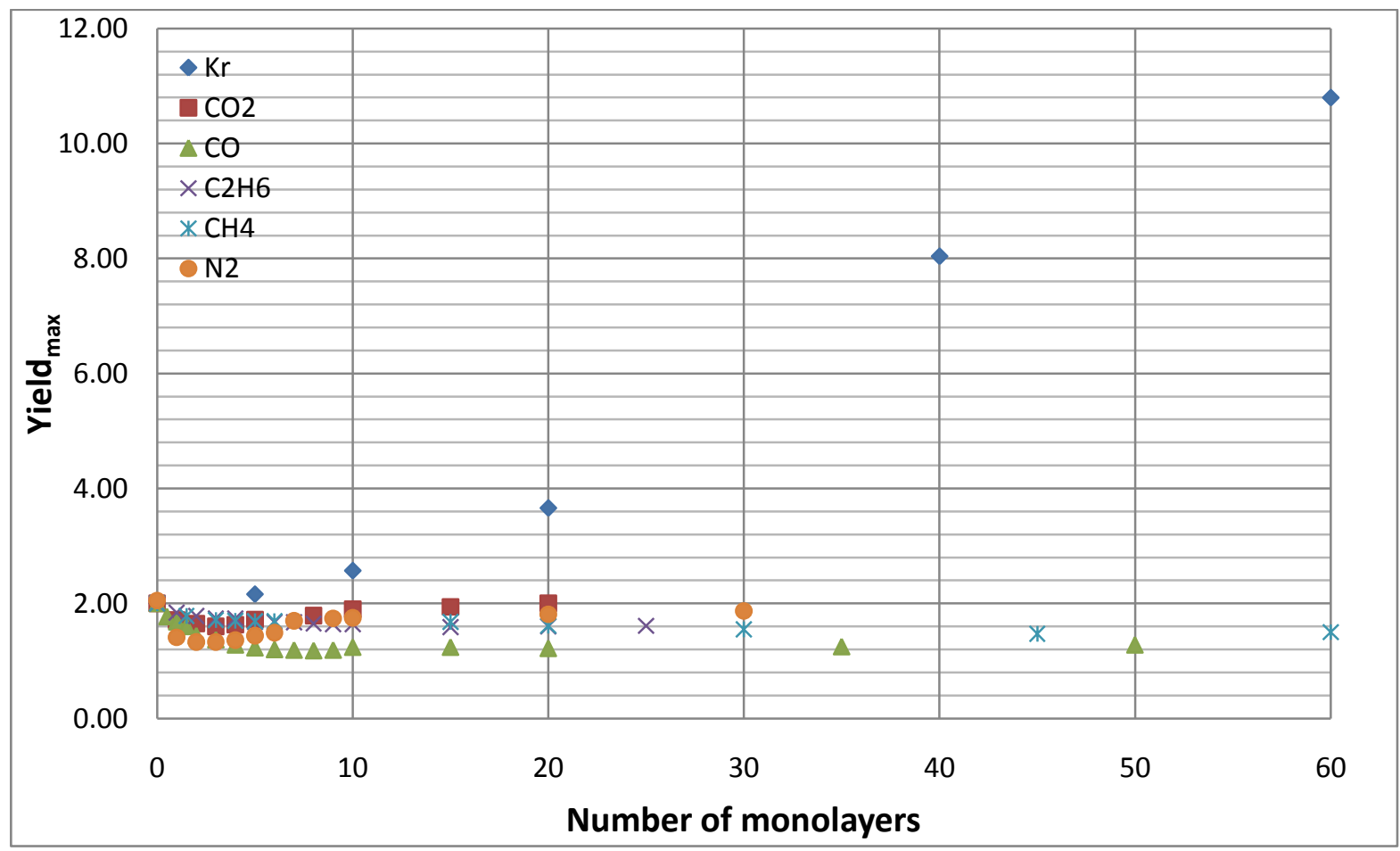

Figure 5.19: Maximum yield as a function of coverage for different adsorbates. 


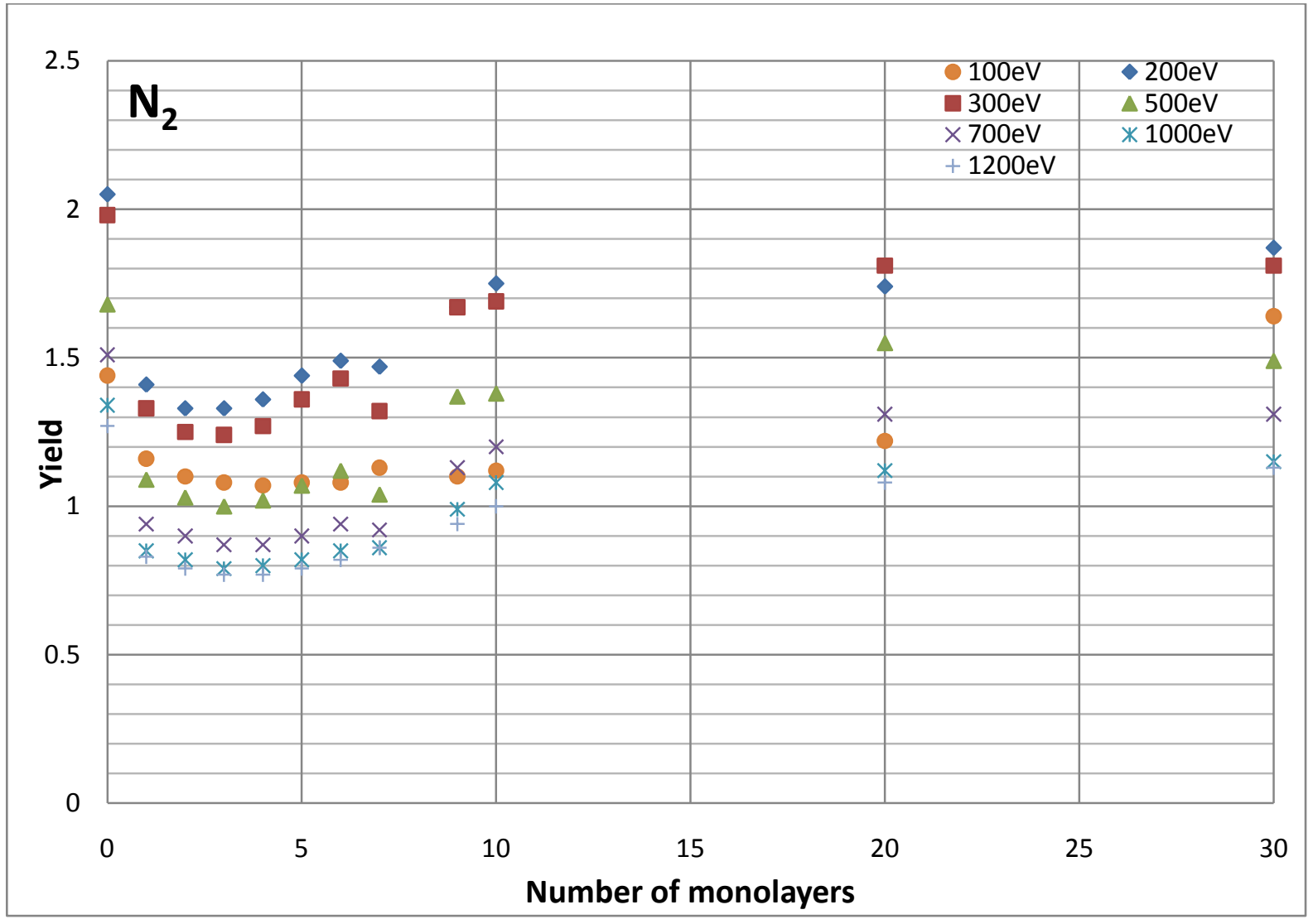

Figure 5.20: SEY of $\mathrm{N}_{2}$ as a function of coverage for defined primary electron energies.

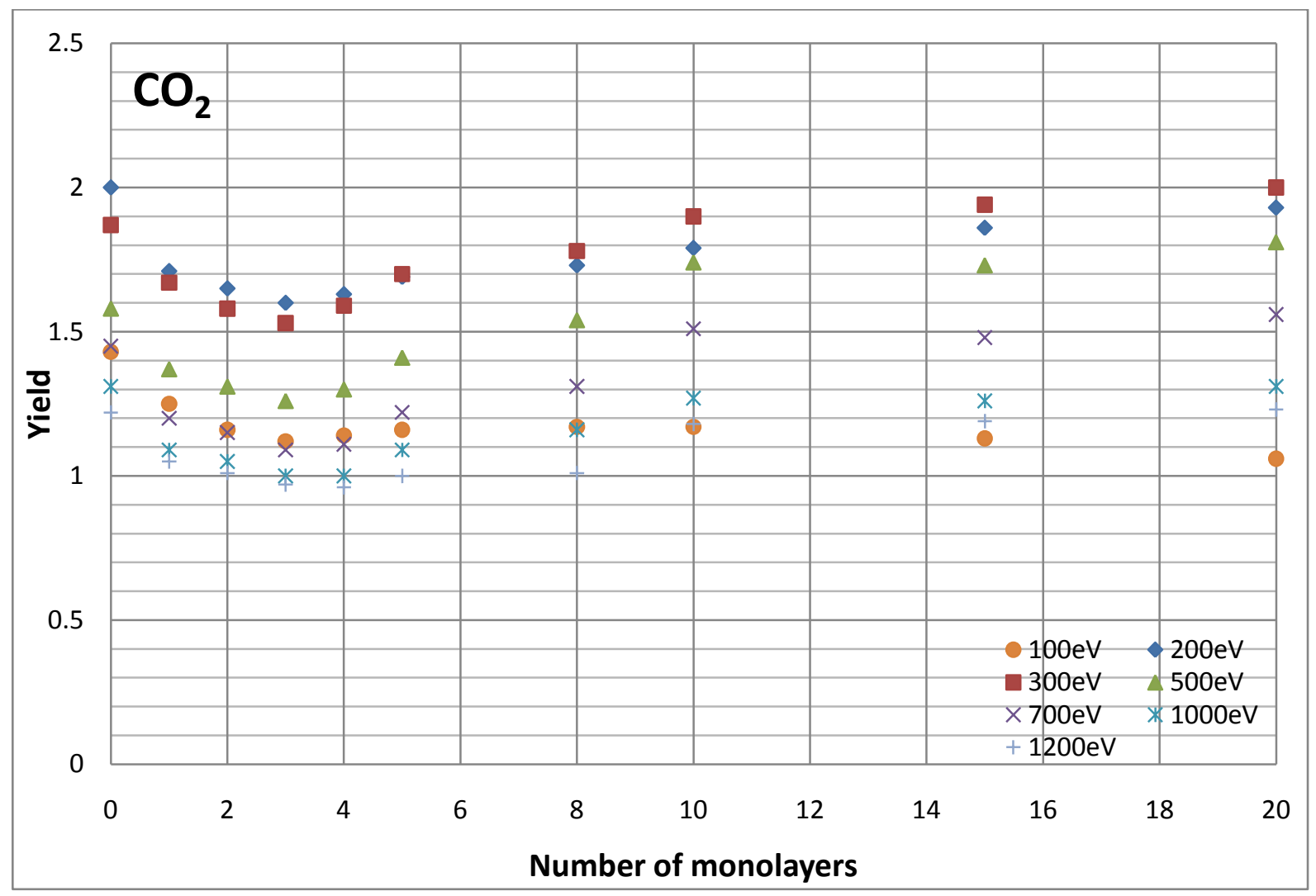

Figure 5.21: $\mathrm{SEY}$ of $\mathrm{CO}_{2}$ as a function of coverage for defined primary electron energies. 
Chapter 5-Results

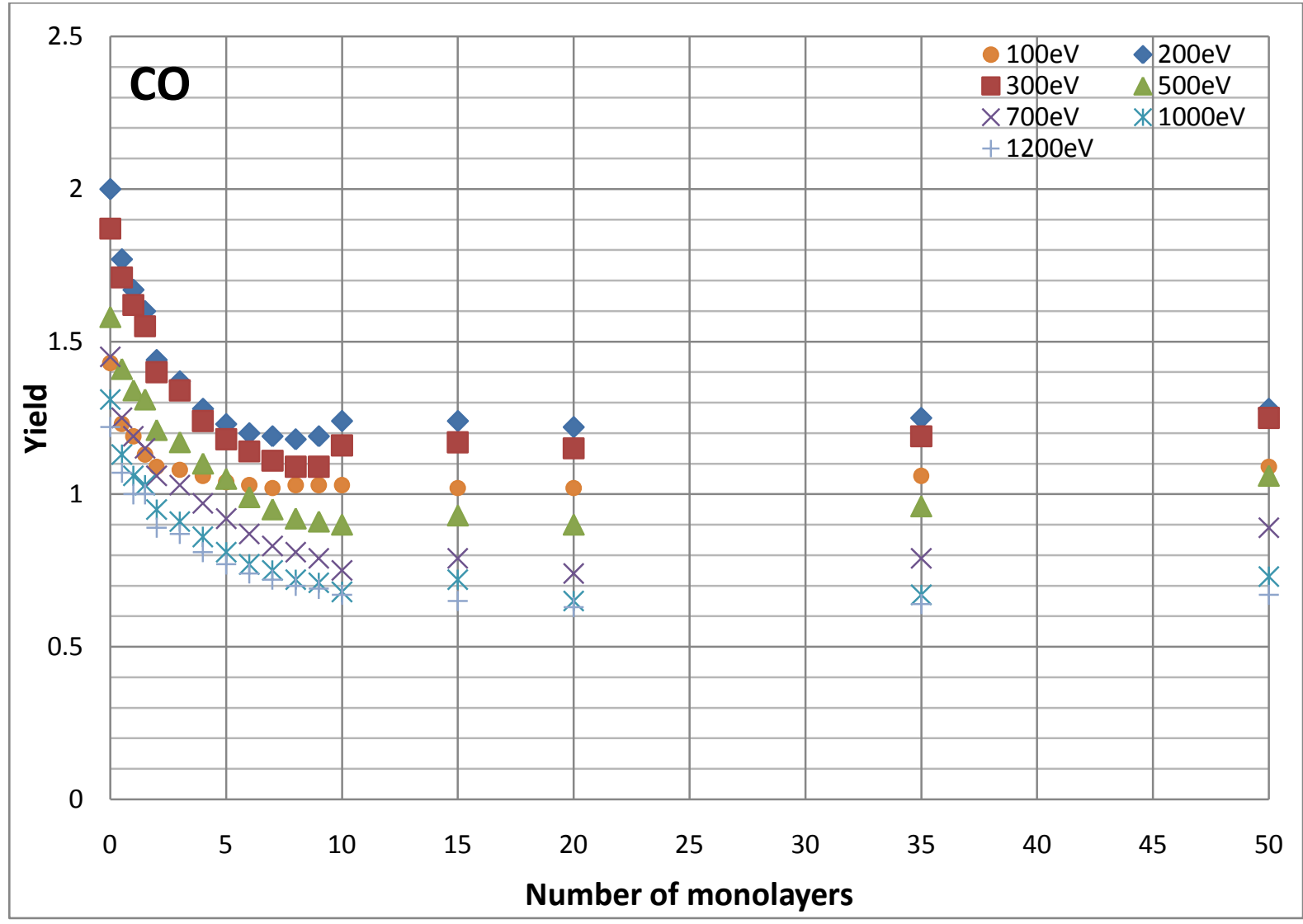

Figure 5.22: SEY of CO as a function of coverage for defined primary electron energies.

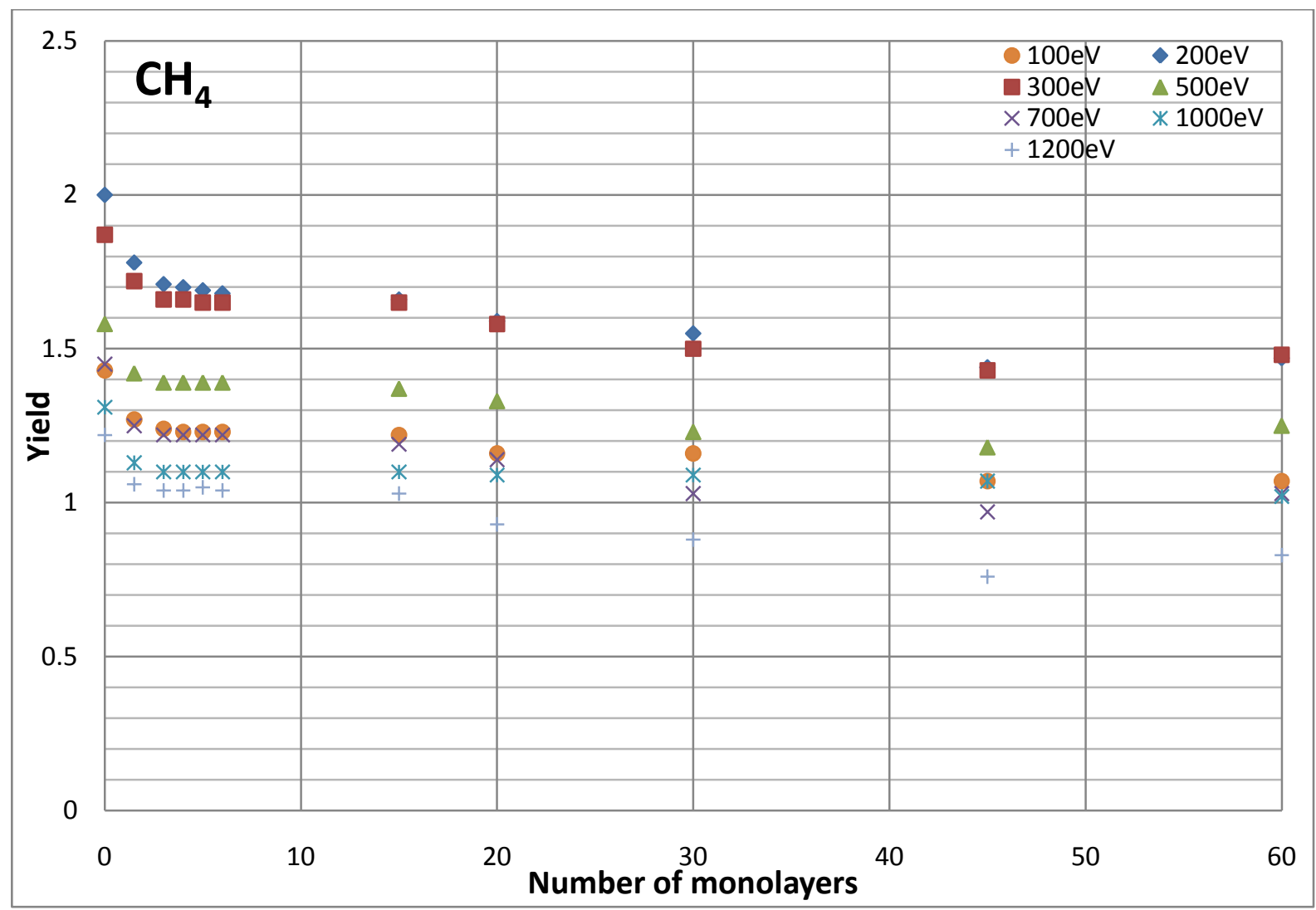

Figure 5.23: $\mathrm{SEY}$ of $\mathrm{CH}_{4}$ as a function of coverage for defined primary electron energies. 
Chapter 5-Results

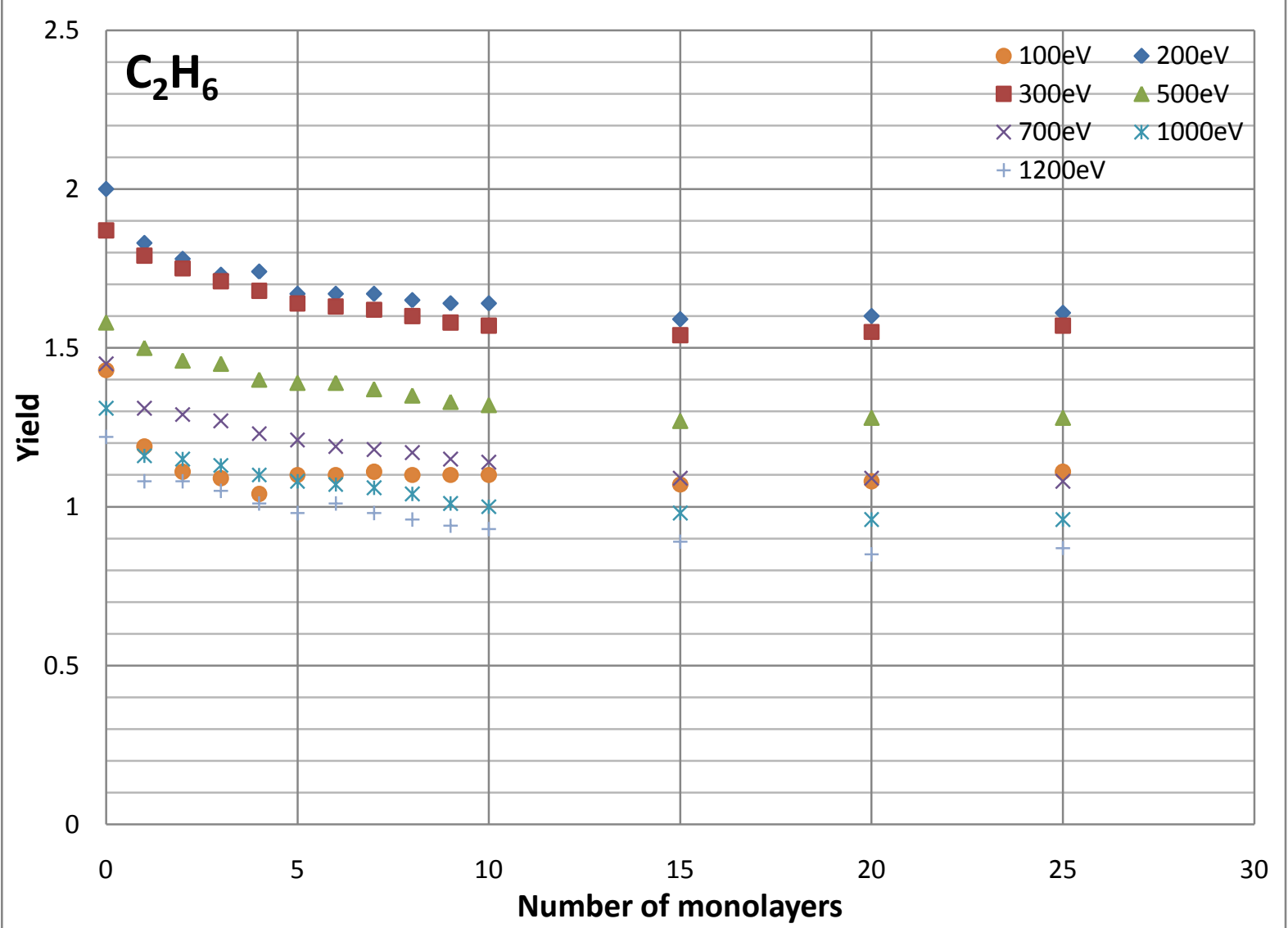

Figure 5.24: $\mathrm{SEY}$ of $\mathrm{C}_{2} \mathrm{H}_{6}$ as a function of coverage for defined primary electron energies.

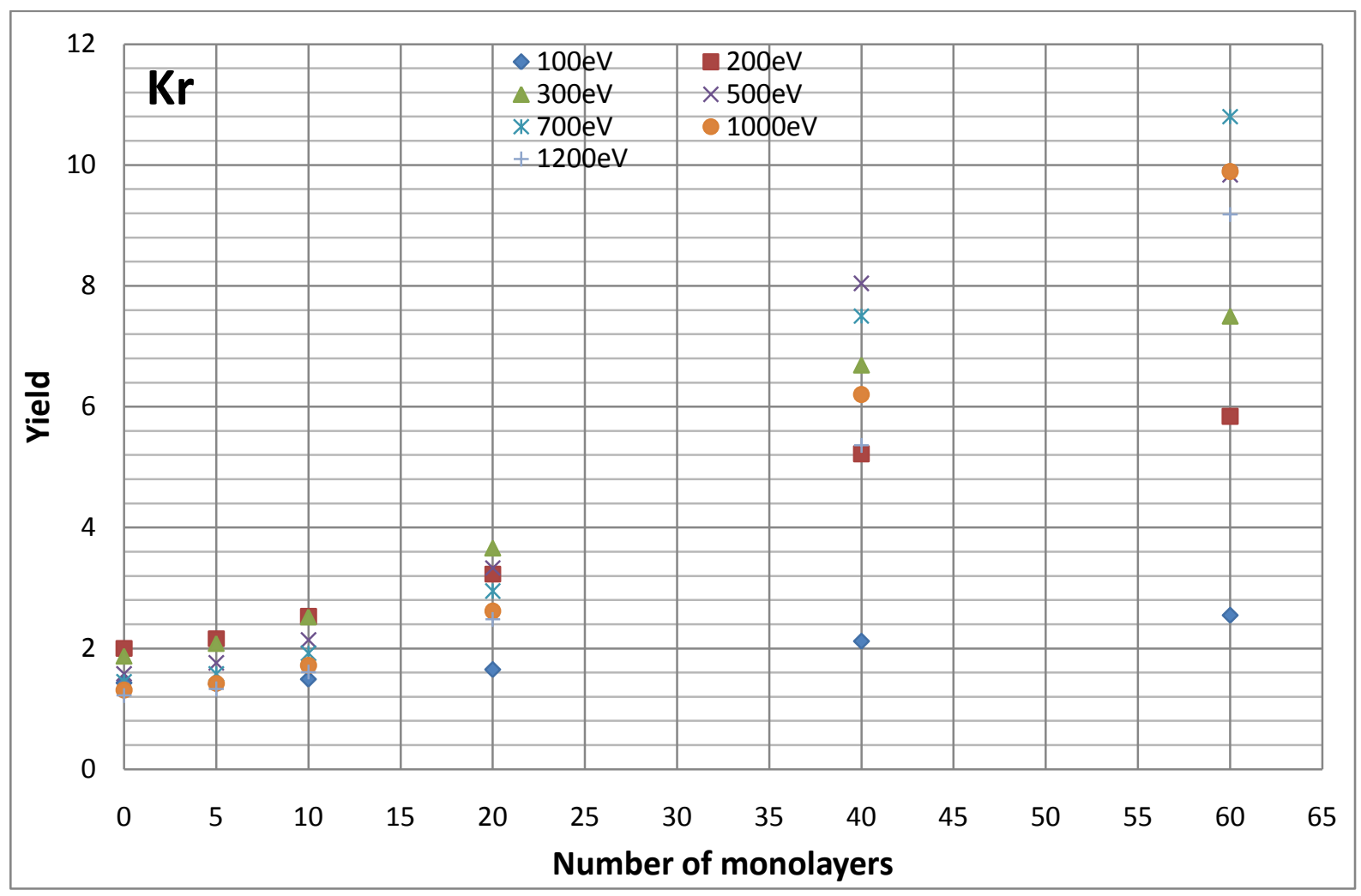

Figure 5.25: SEY of Kr as a function of coverage for defined primary electron energies. 


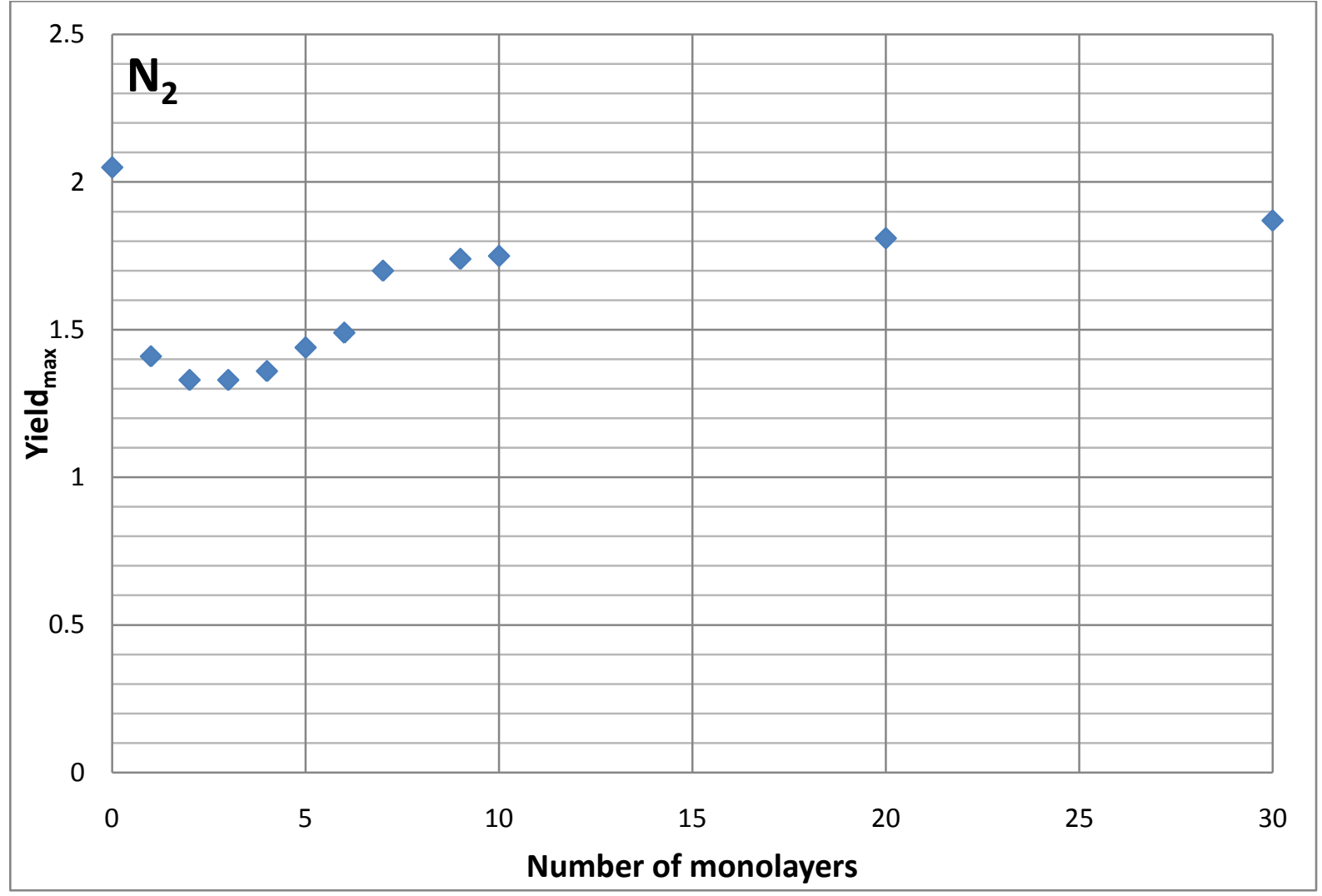

Figure 5.26: Maximum yield of $\mathrm{N}_{2}$ for each number of monolayer.

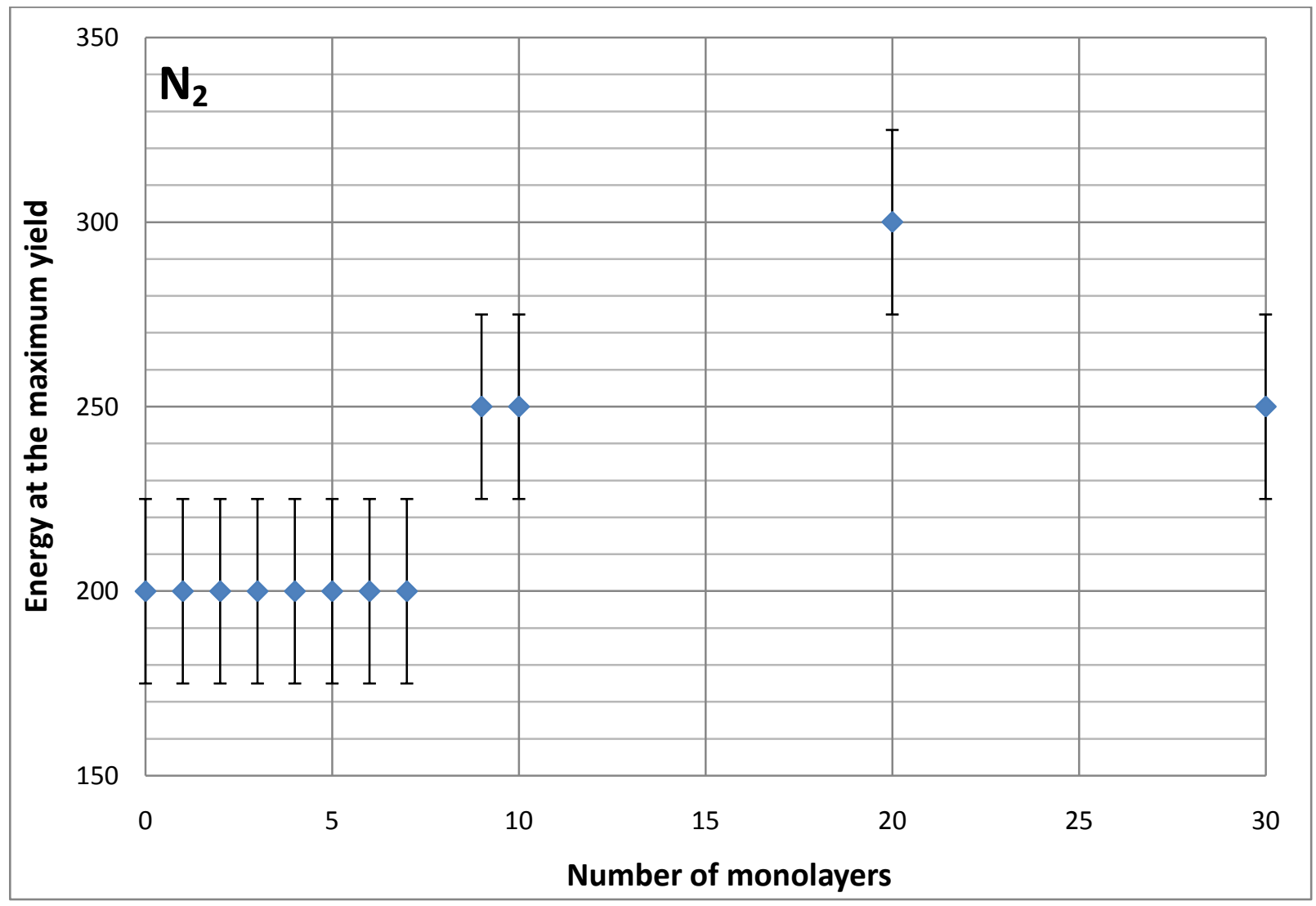

Figure 5.27: Energy at the maximum yield of $\mathrm{N}_{2}$ as a function of coverage. 


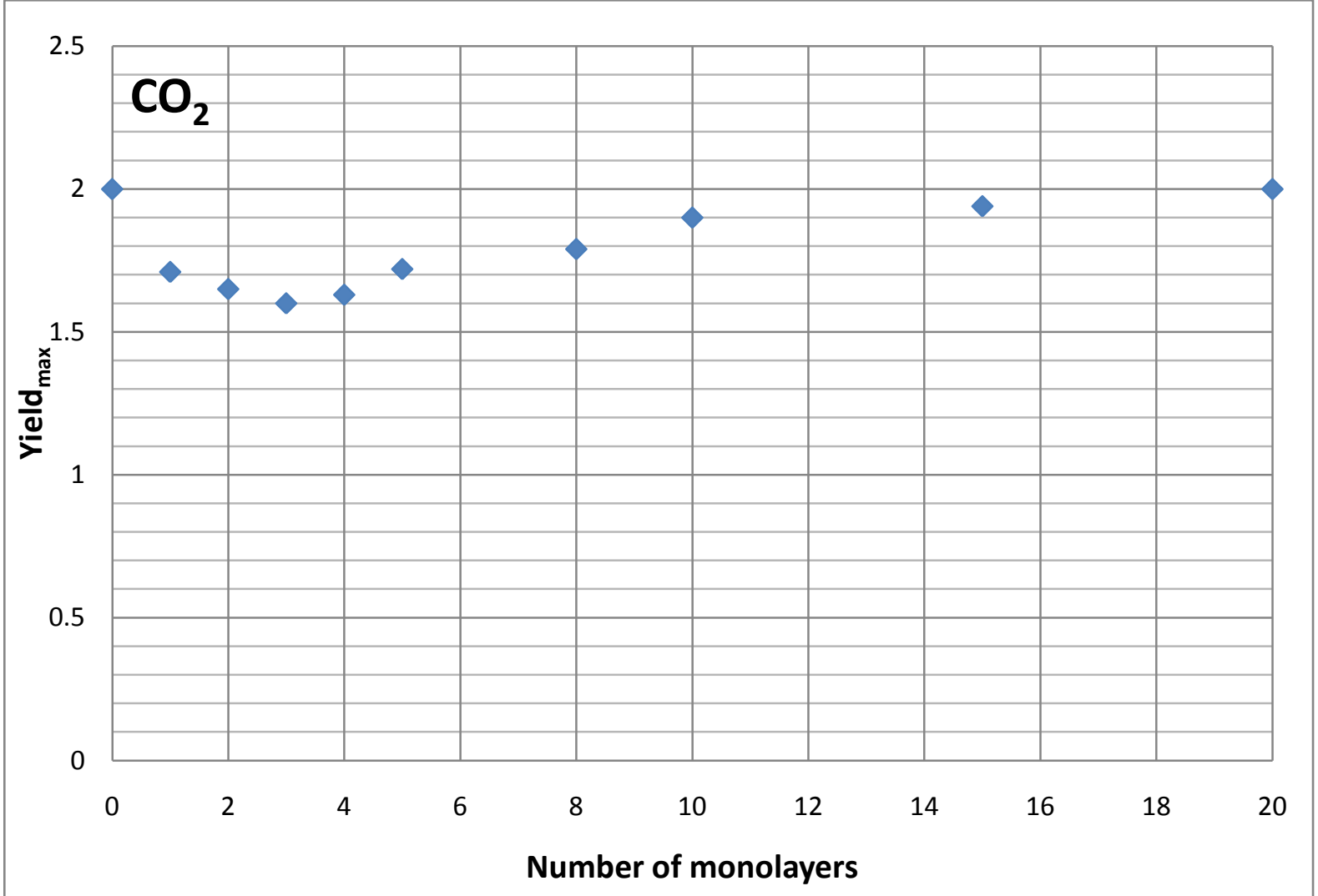

Figure 5.28: Maximum yield of $\mathrm{CO}_{2}$ for each number of monolayer.

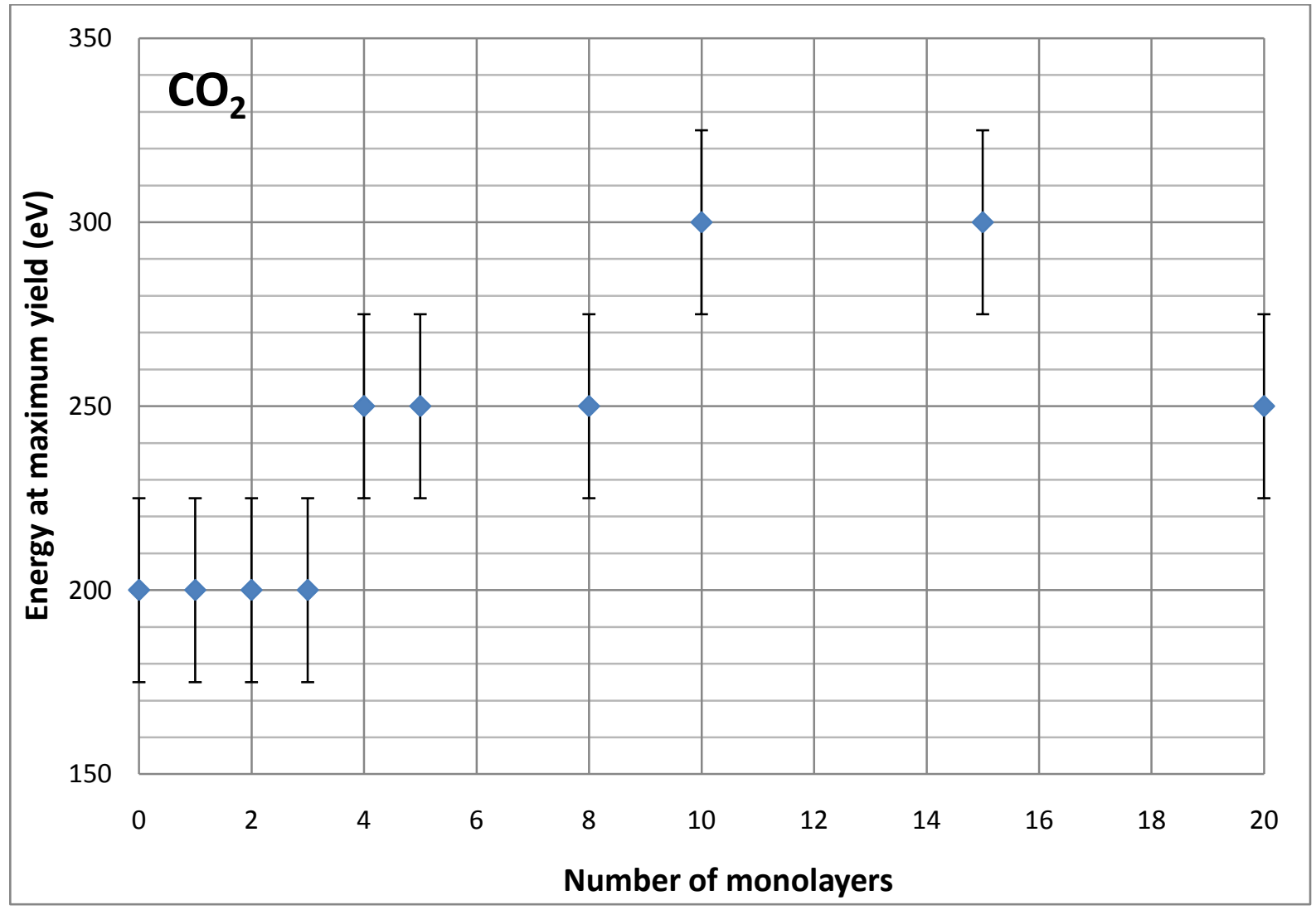

Figure 5.29: Energy at the maximum yield of $\mathrm{CO}_{2}$ as a function of coverage. 


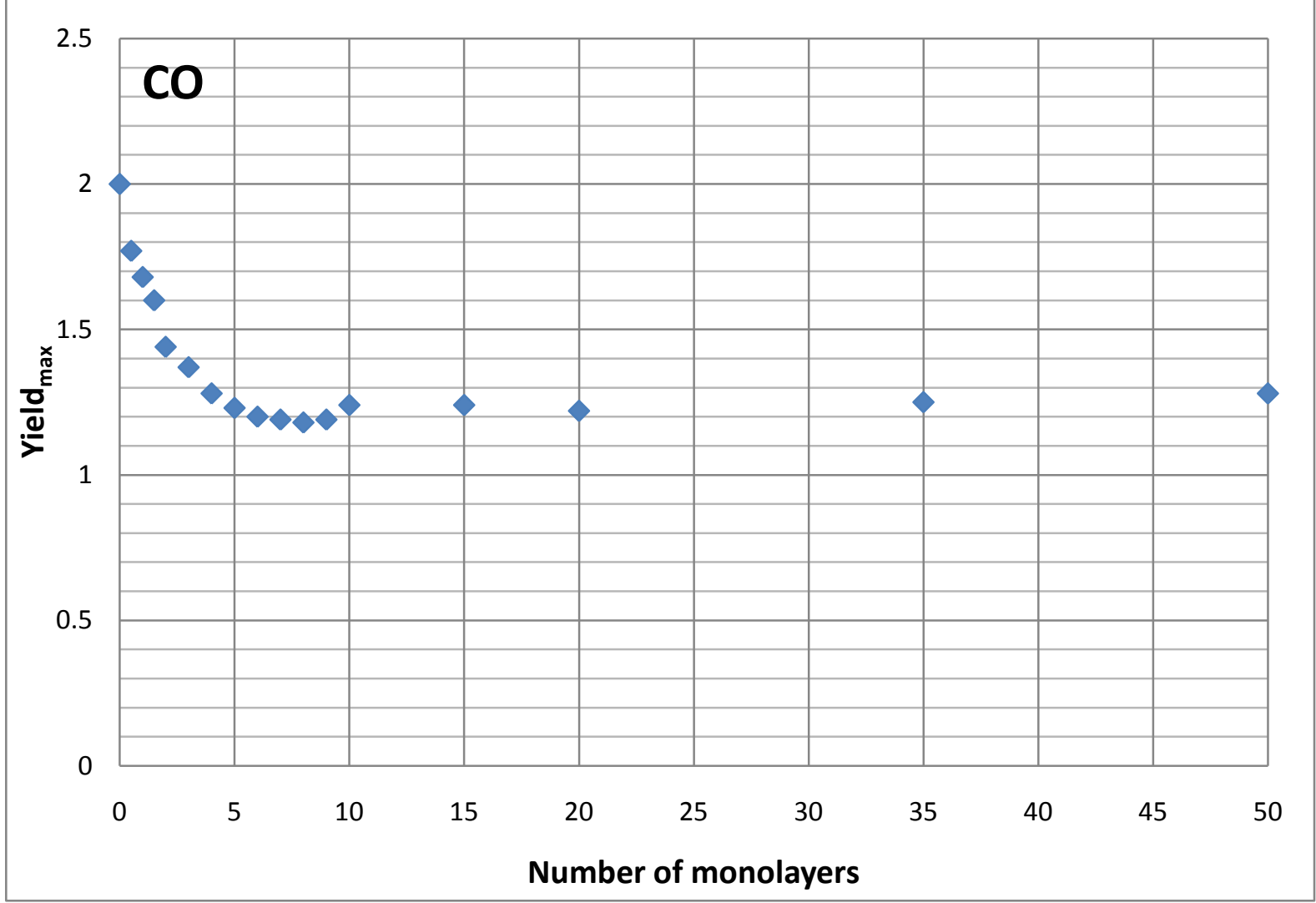

Figure 5.30: Maximum yield of CO for each number of monolayer.

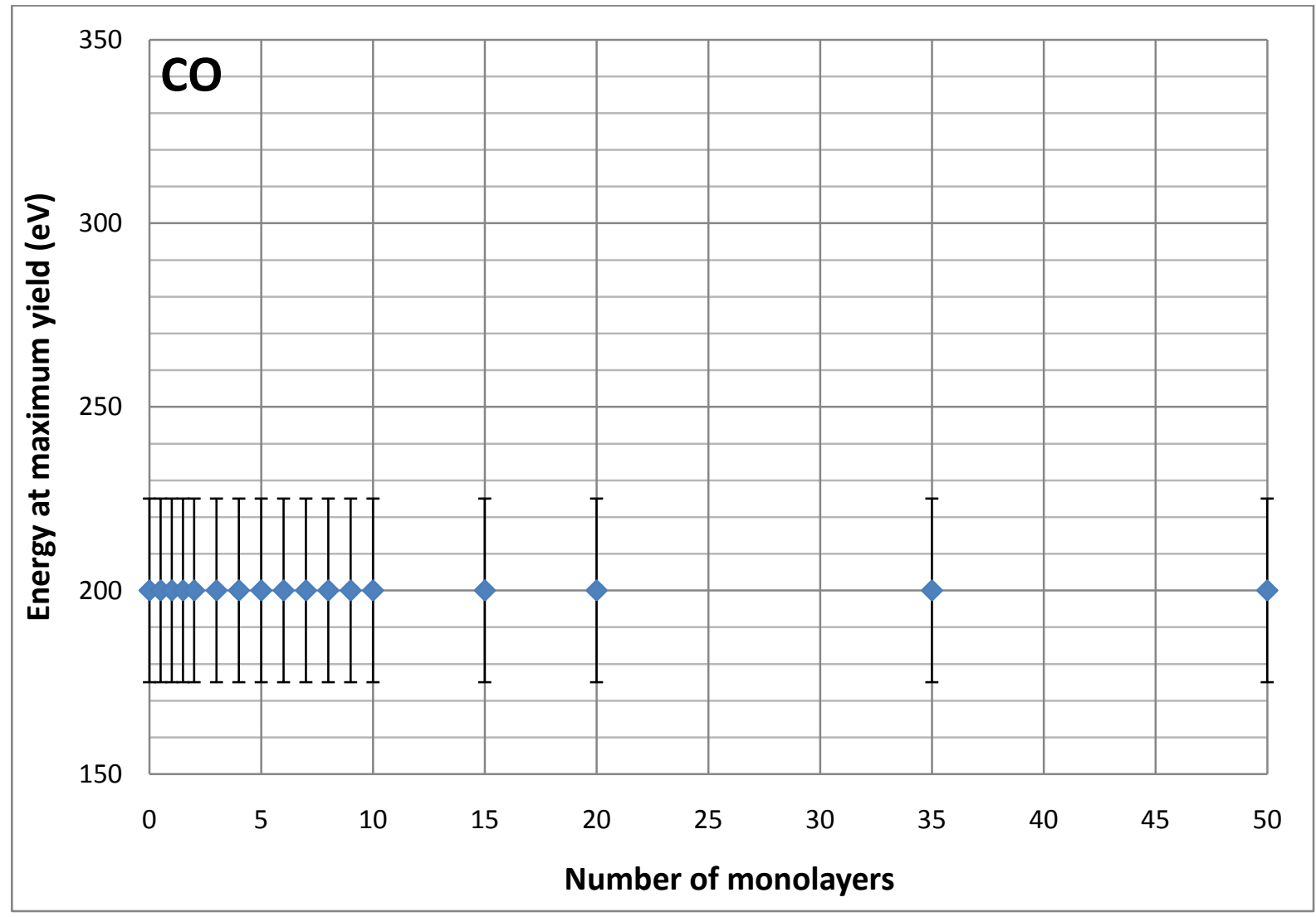

Figure 5.31: Energy at the maximum yield of $\mathrm{CO}$ as a function of coverage. 


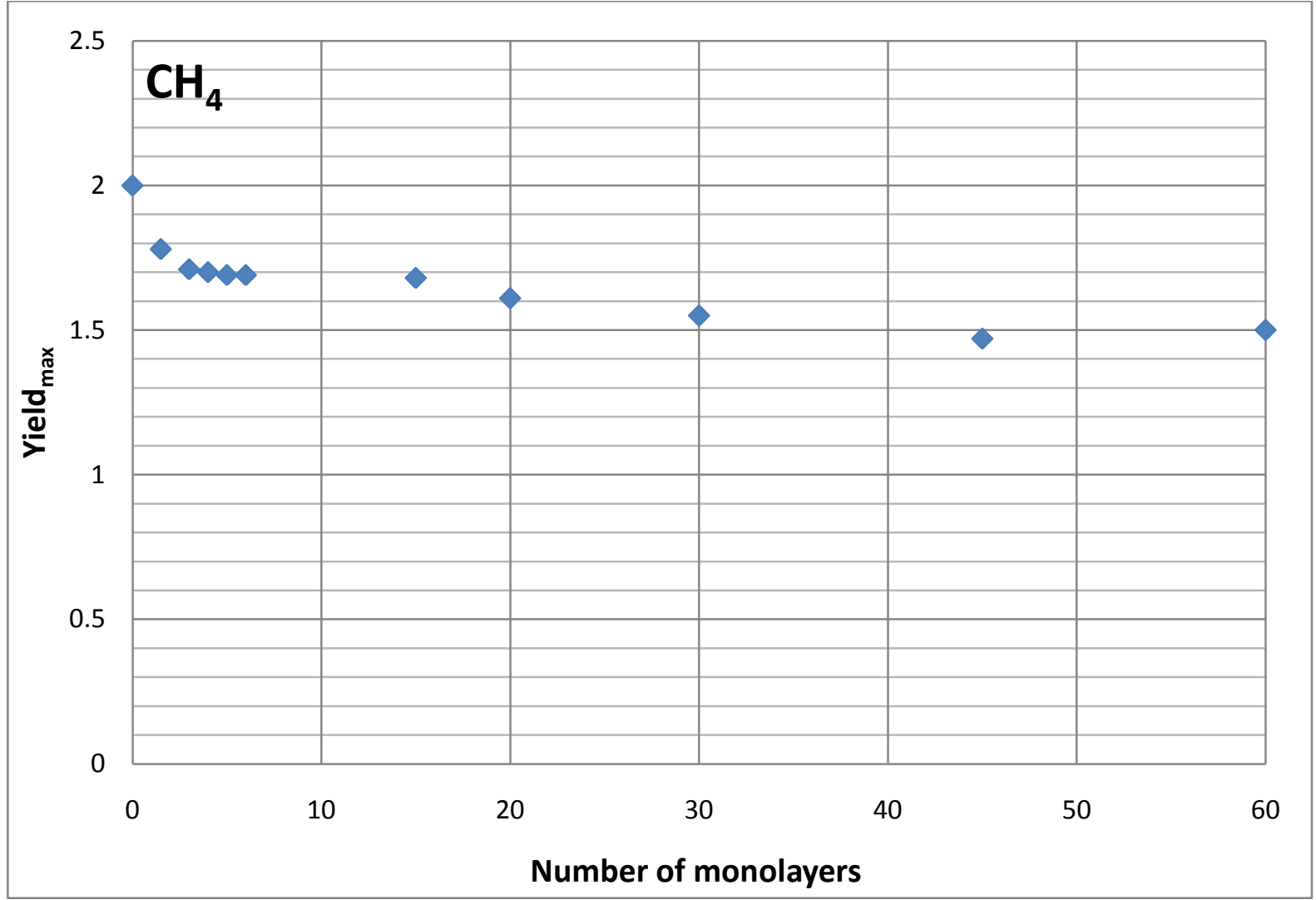

Figure 5.32: Maximum yield of $\mathrm{CH}_{4}$ for each number of monolayer.

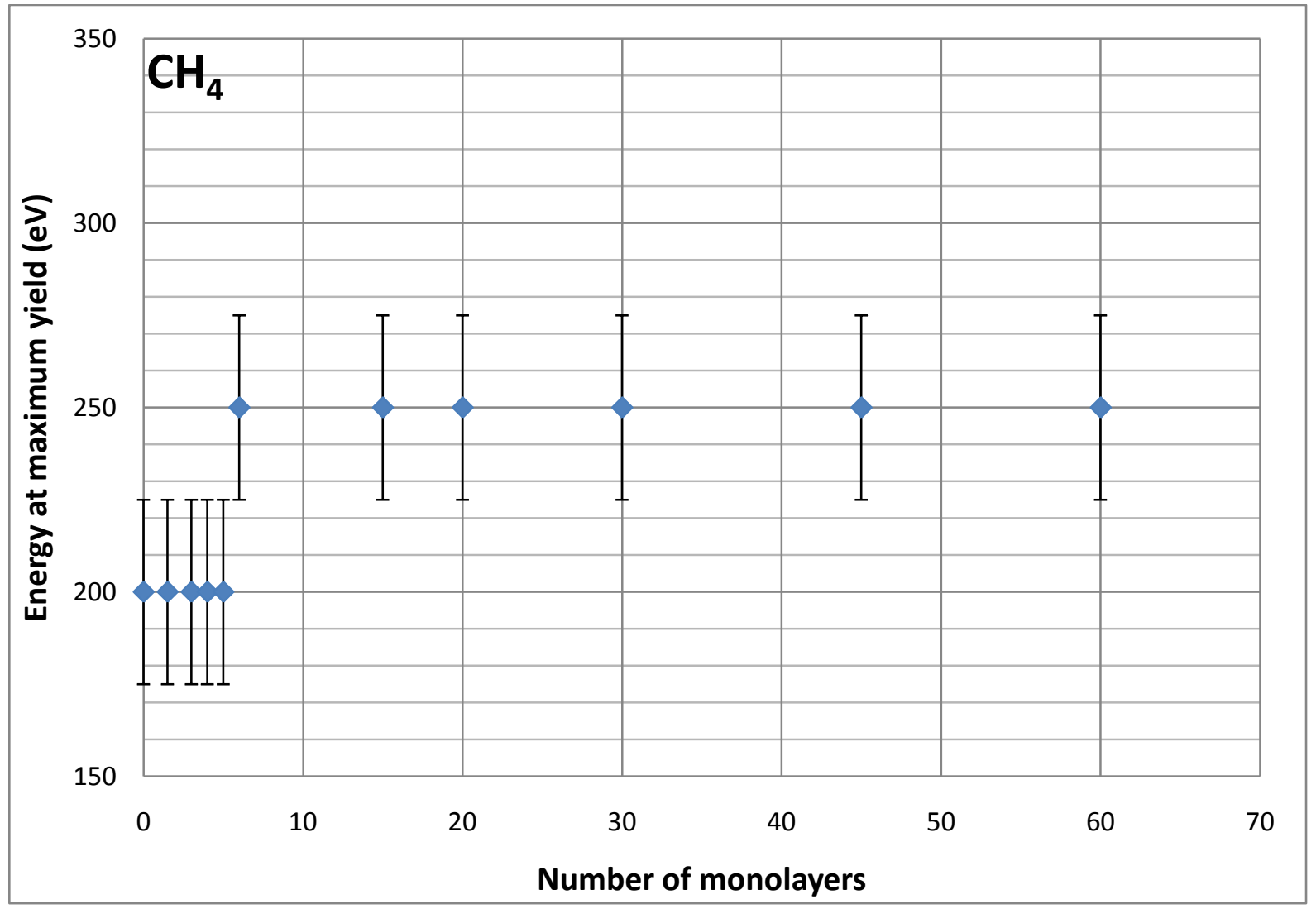

Figure 5.33: Energy at the maximum yield of $\mathrm{CH}_{4}$ as a function of coverage. 


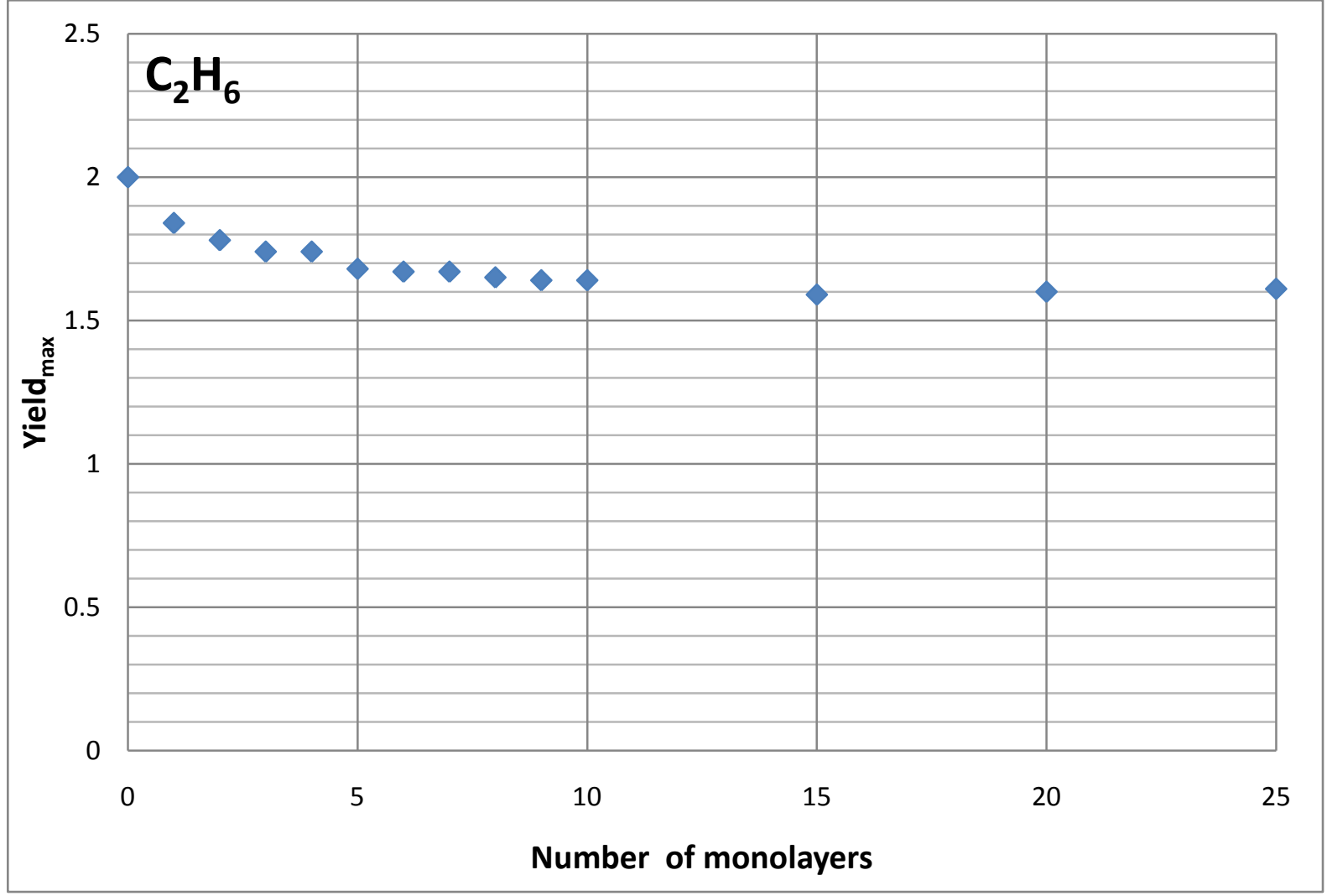

Figure 5.34: Maximum yield of $\mathrm{C}_{2} \mathrm{H}_{6}$ for each number of monolayer.

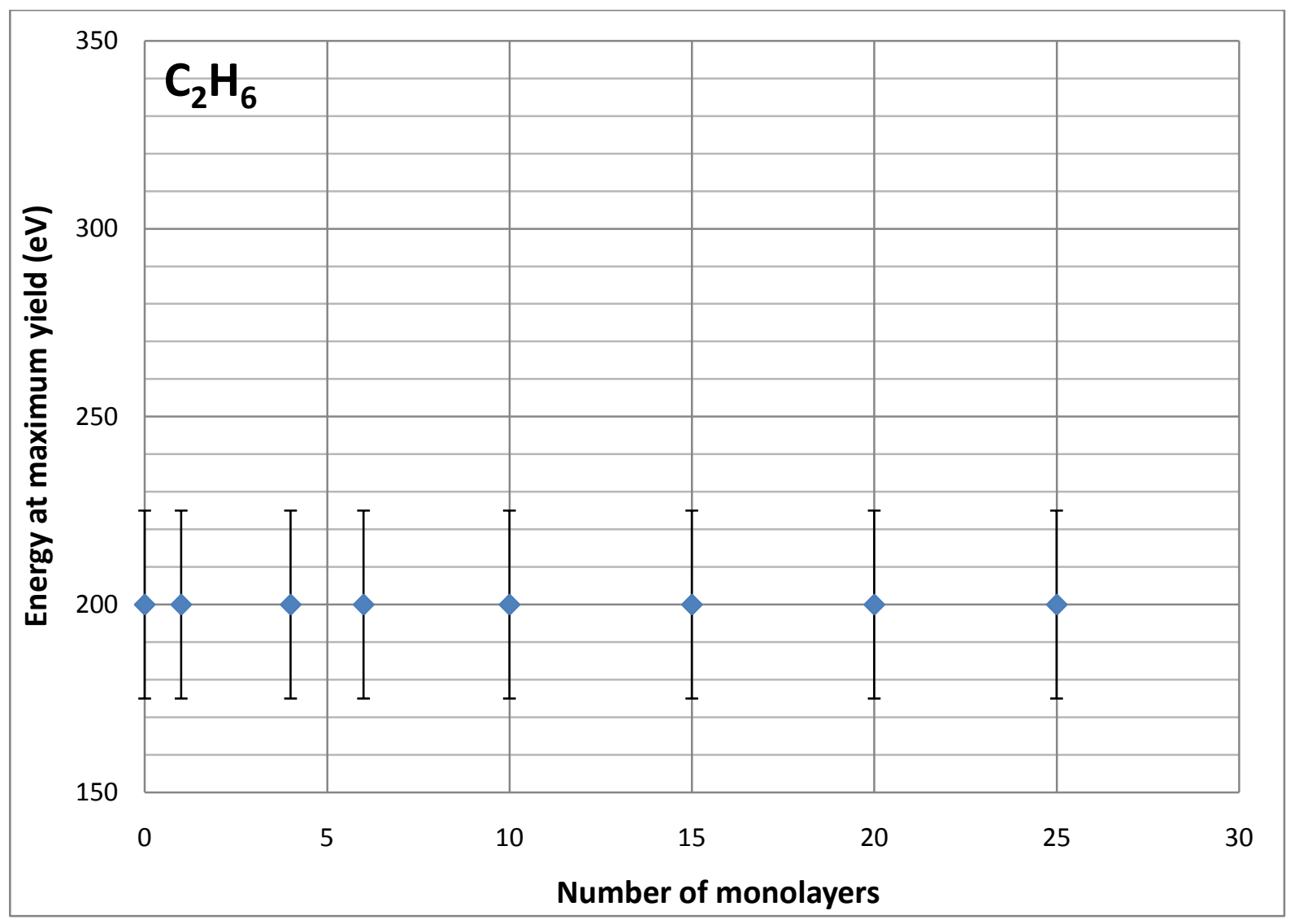

Figure 5.35: Energy at the maximum yield of $\mathrm{C}_{2} \mathrm{H}_{6}$ as a function of coverage. 


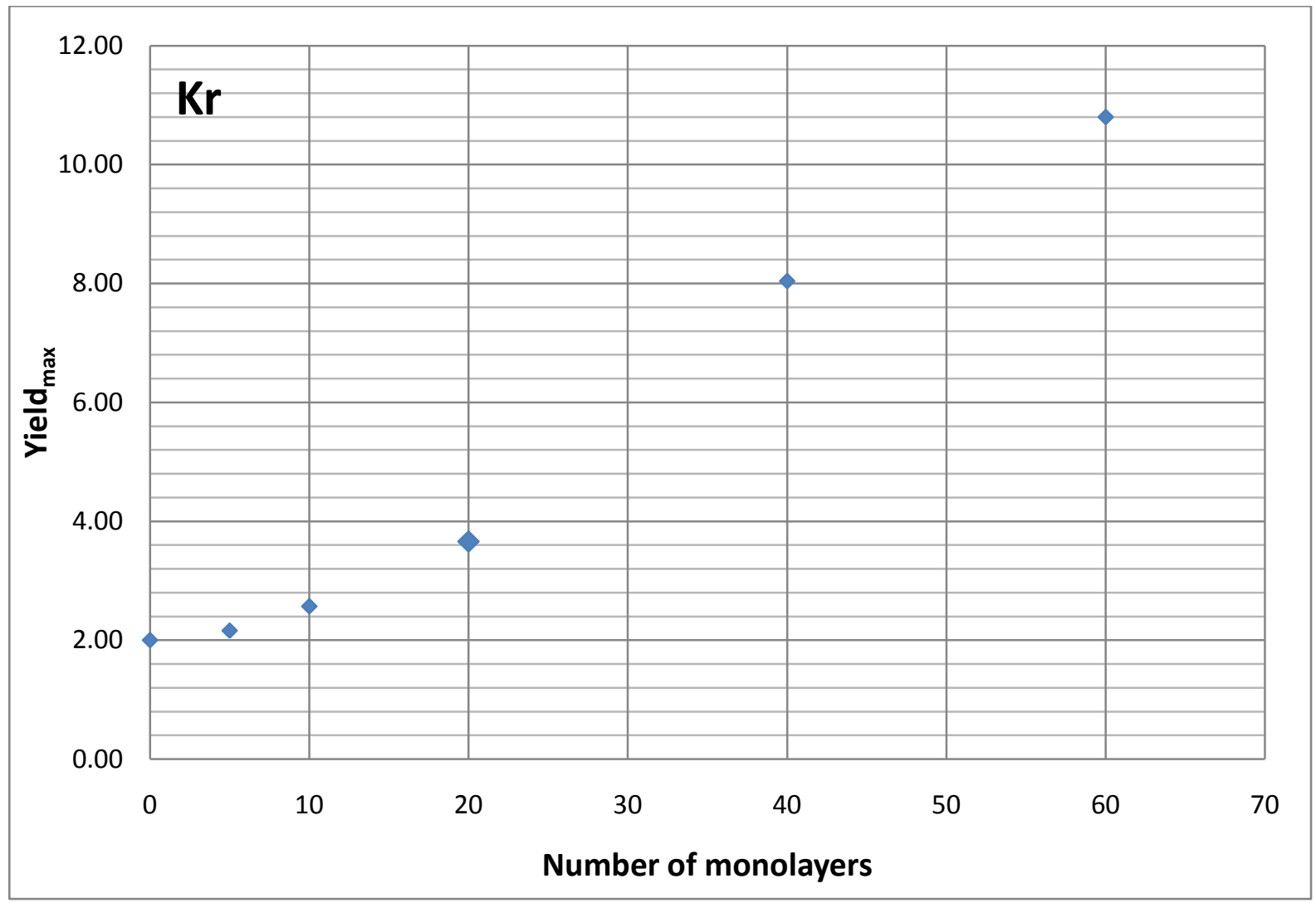

Figure 5.36: Maximum yield of $\mathrm{Kr}$ for each number of monolayer.

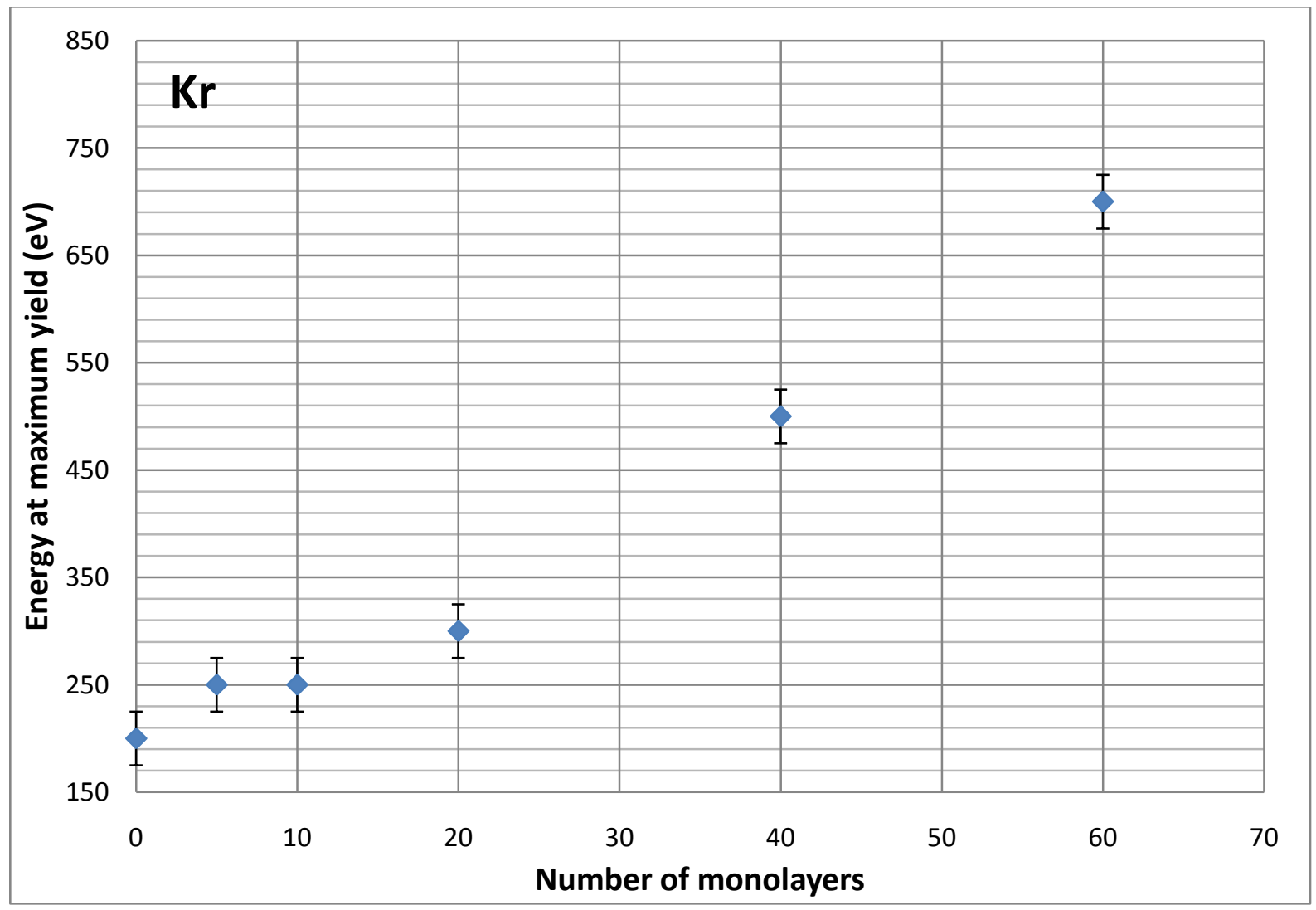

Figure 5.37: Energy at the maximum yield of $K r$ as a function of coverage. 


\subsubsection{SEY of Aluminium}

To understand more about the influence of the effective surface area of different materials on the secondary electron yield and the importance of the substrate yield in the total yield with adsorbates, measurements with different substrates have been performed. The second substrate used for the measurement was aluminium. Aluminium has a larger secondary electron yield than copper. At room temperature the maximum yield is 3.66 and at cryogenic temperatures it is about 3.04. In figure 5.38, we see that the yield decreases when we go down to cryogenic temperatures as in the case of copper.

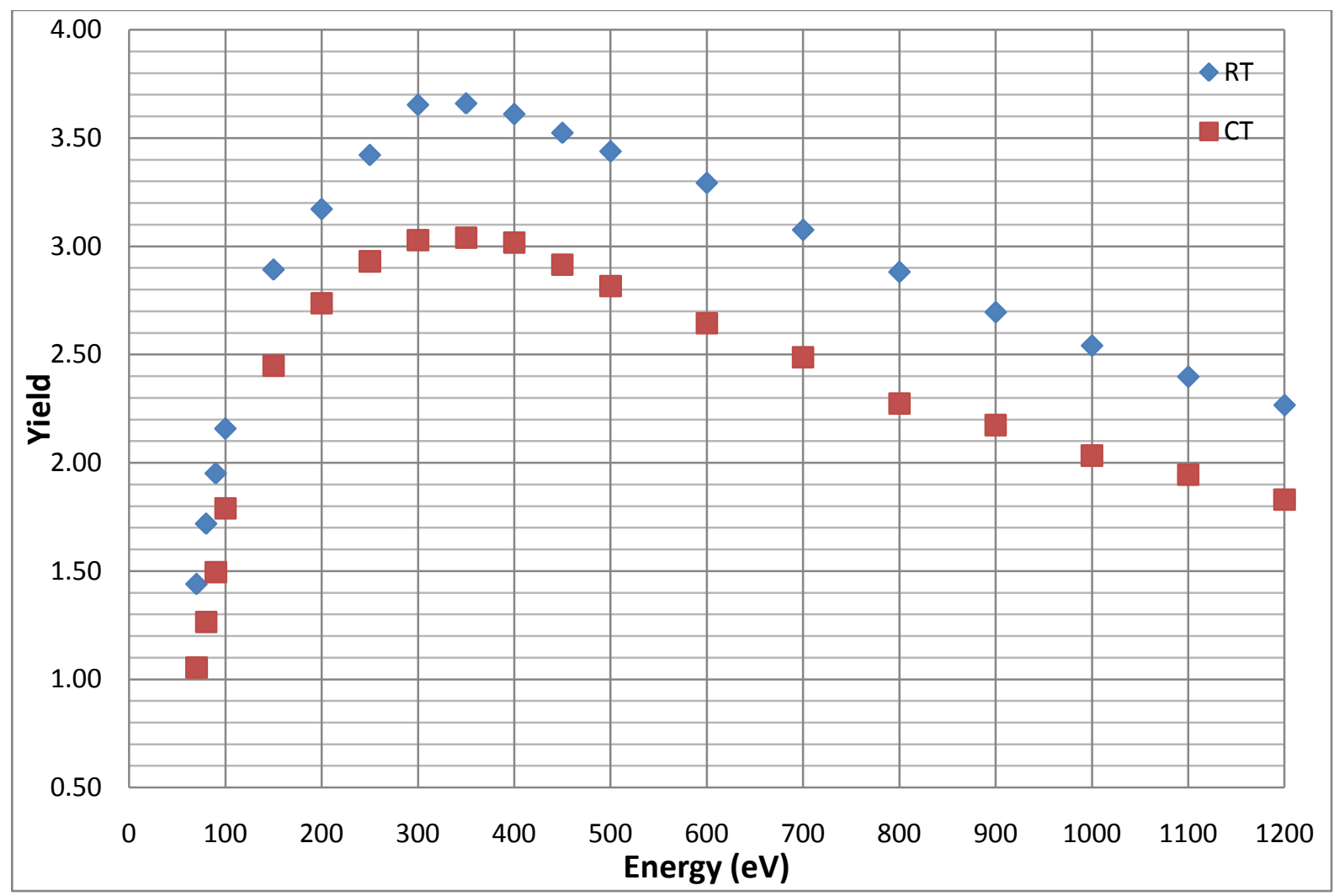

Figure 5.38: Secondary electron yield of aluminium at room temperature and at cryogenic temperatures.

\subsubsection{SEY of aluminium as a function of, $\mathrm{N}_{2}, \mathrm{CO}_{2}, \mathrm{CO}$ and $\mathrm{CH}_{4}$ adsorption}

The variation of the SEY as a function of 10 monolayers $\mathrm{N}_{2}, \mathrm{CO}_{2}, \mathrm{CO}$ and $\mathrm{CH}_{4}$ physisorbed on the aluminium is shown in figure 5.39 .

It can be observed that the adsorption of 10 monolayers of each gas decreases the yield. 


\section{Chapter 5-Results}

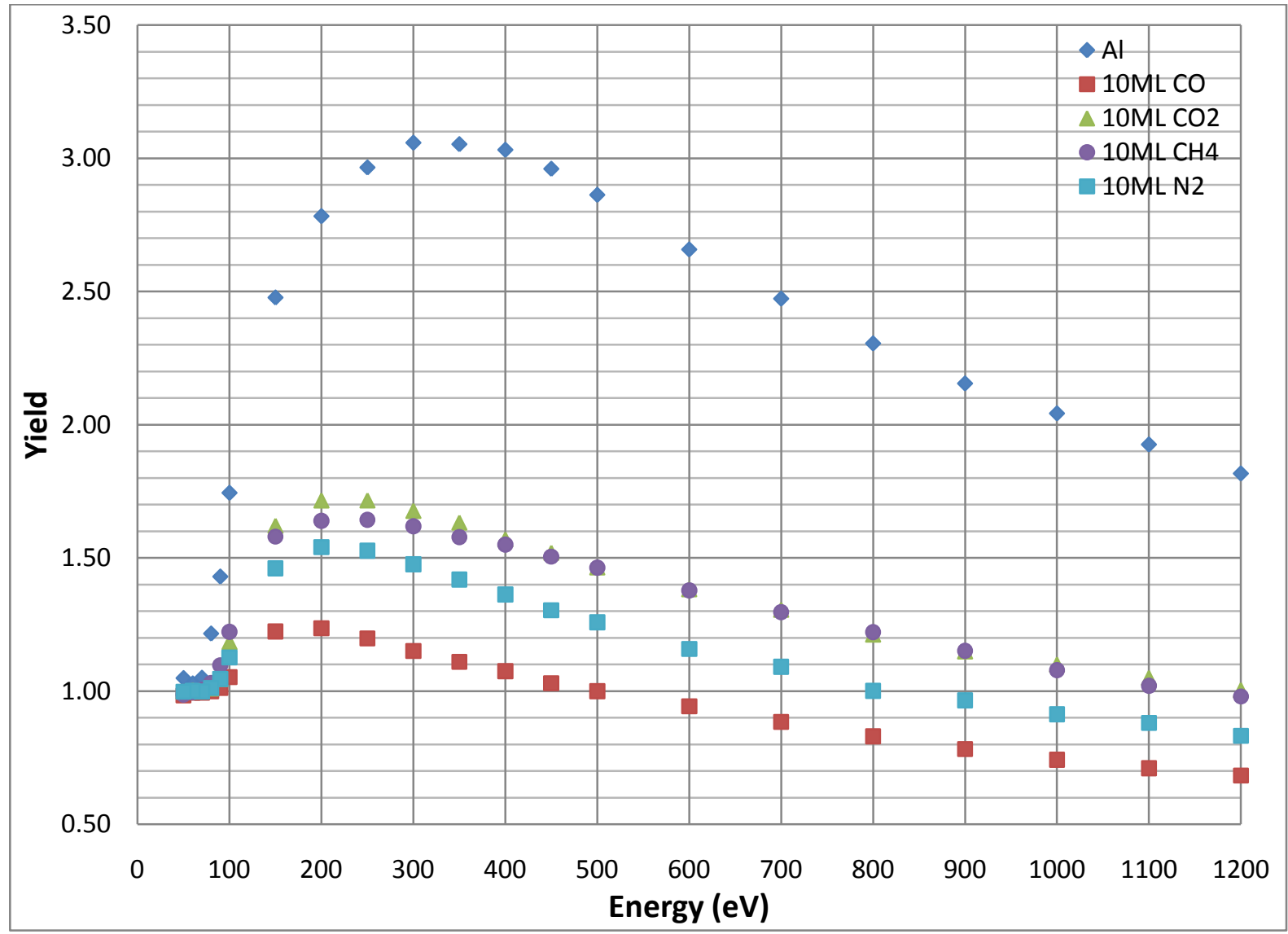

Figure 5.39: The secondary electron yield of 10 monolayers of $\mathrm{N}_{2}, \mathrm{CO}_{2}, \mathrm{CO}$ and $\mathrm{CH}_{4}$ physisorbed on copper.

The energy for maximum yield is different for 10 monolayers of different gases. While $\mathrm{CO}_{2}$ and $\mathrm{CH}_{4}$ have the maximum yield at $250 \mathrm{eV}, \mathrm{CO}$ and $\mathrm{N}_{2}$ have it at $200 \mathrm{eV}$. Aluminium itself has the maximum yield at $300 \mathrm{eV}$. The secondary electron yield for each gas with a different number of monolayers as a function of the primary electron energy is shown in figures 5.40 till 5.47. For each gas the first curves show the SEY for high coverage and the second curves show the SEY with low coverage.

Figures 5.48, 5.49, 5.50, and 5.51 show the secondary electron yield as a function of the coverage for some selected primary electron energies.

The maximum yield at different numbers of coverage for each gas and the primary energy at the maximum yield as a function of coverage are shown in figure 5.52 to 5.59 .

In the last figure (Figure 5.60) the maximum yield of all the gases used for the measurements are shown in one single graph as a function of coverage. 
Chapter 5-Results

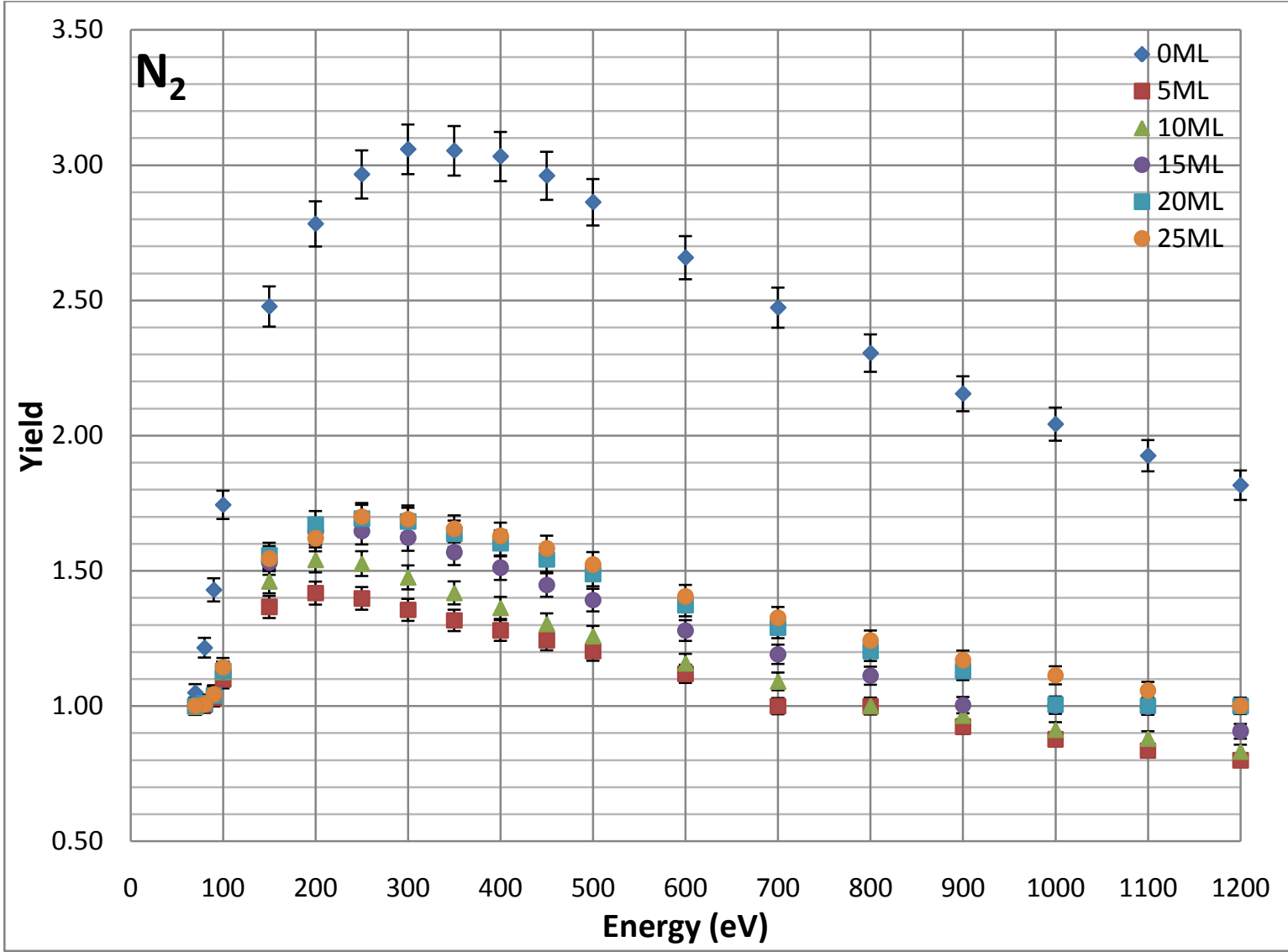

Figure 5.40: SEY of adsorbed $\mathrm{N}_{2}$ as a function of energy for different coverages.

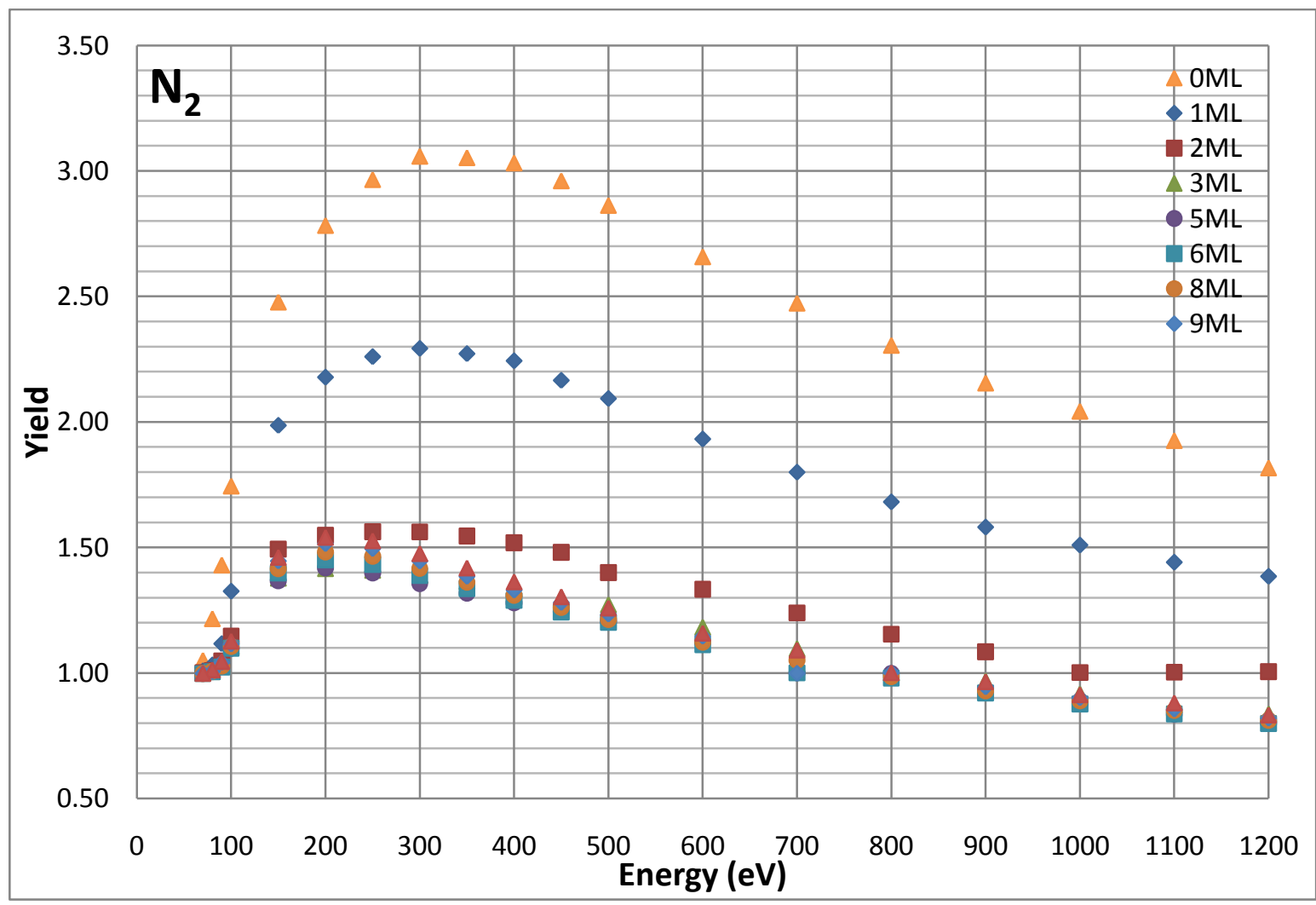

Figure 5.41: SEY of adsorbed $N_{2}$ as a function of energy for low coverage. 
Chapter 5-Results

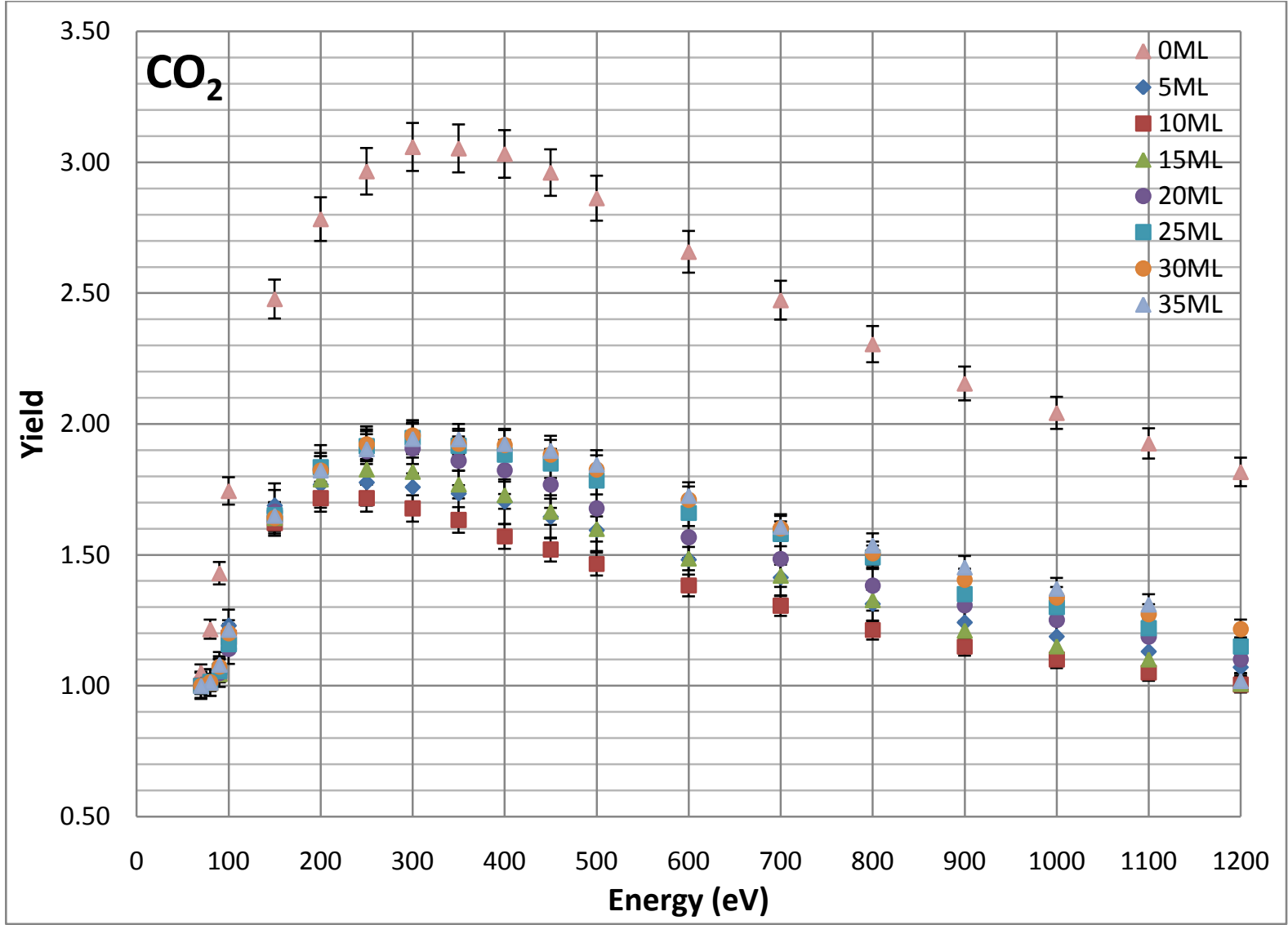

Figure 5.42: SEY of adsorbed $\mathrm{CO}_{2}$ as a function of energy for different coverages.

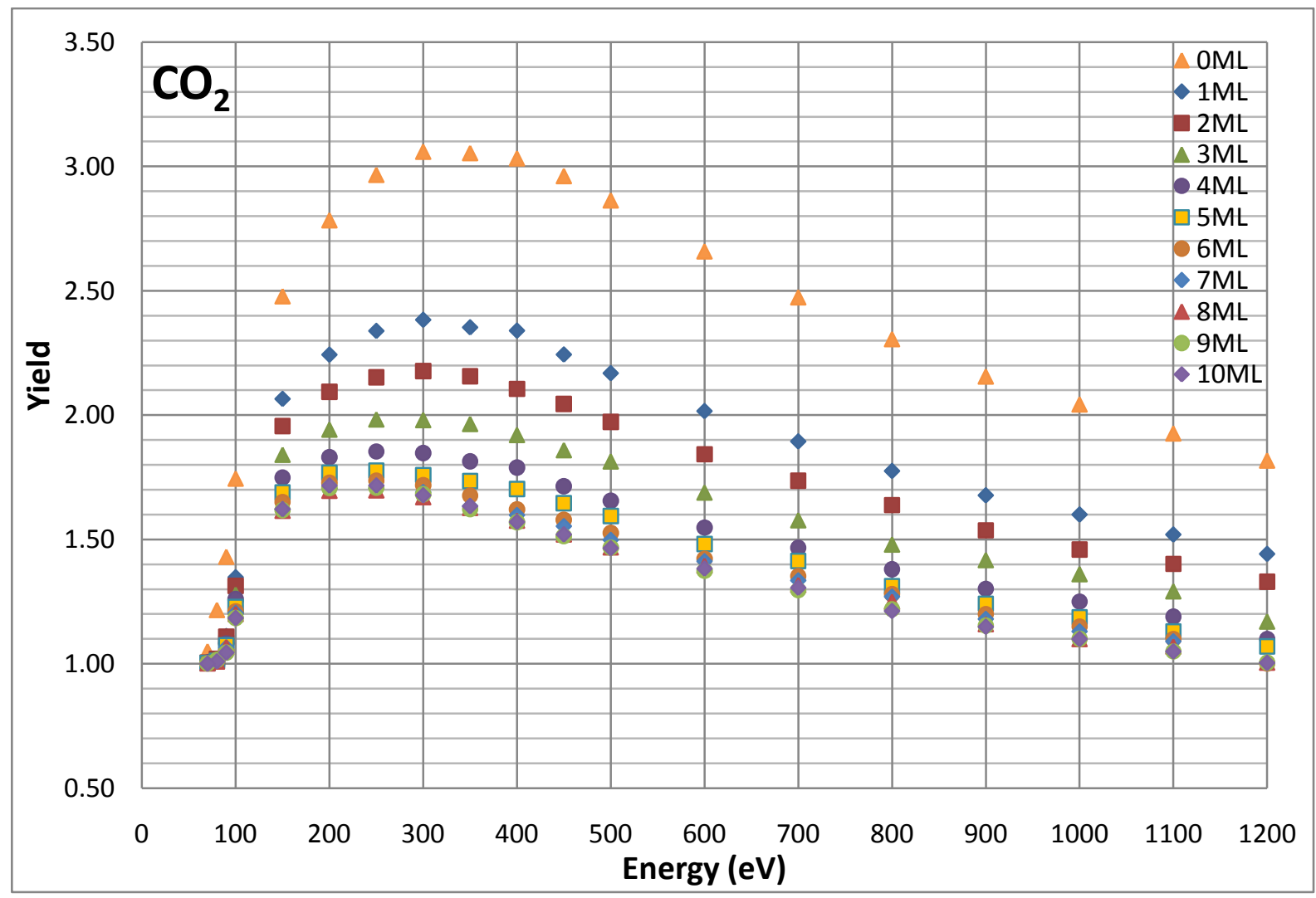

Figure 5.43: $\mathrm{SEY}$ of adsorbed $\mathrm{CO}_{2}$ as a function of energy for low coverage. 


\section{Chapter 5-Results}

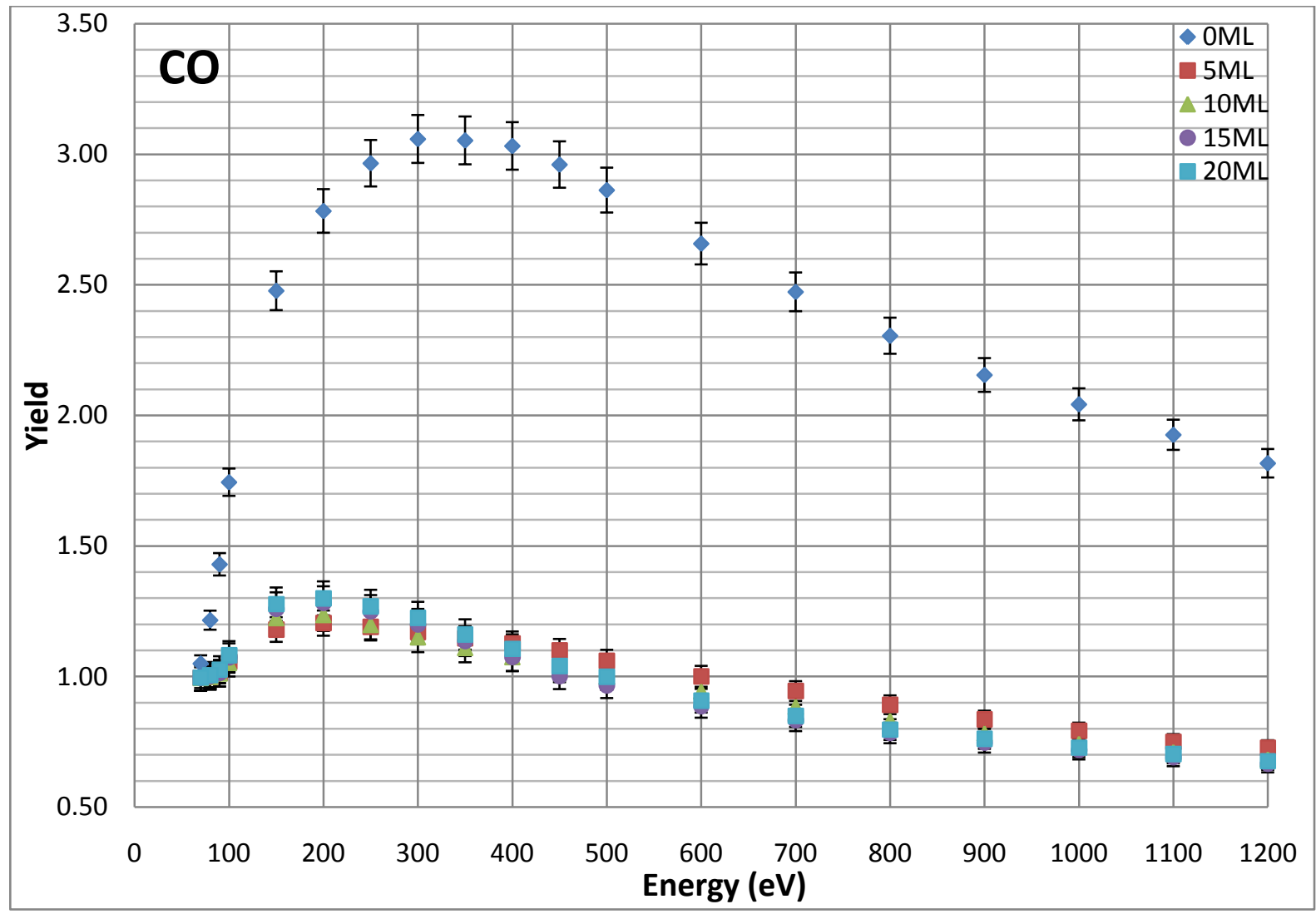

Figure 5.44: SEY of adsorbed CO as a function of energy for different coverages.

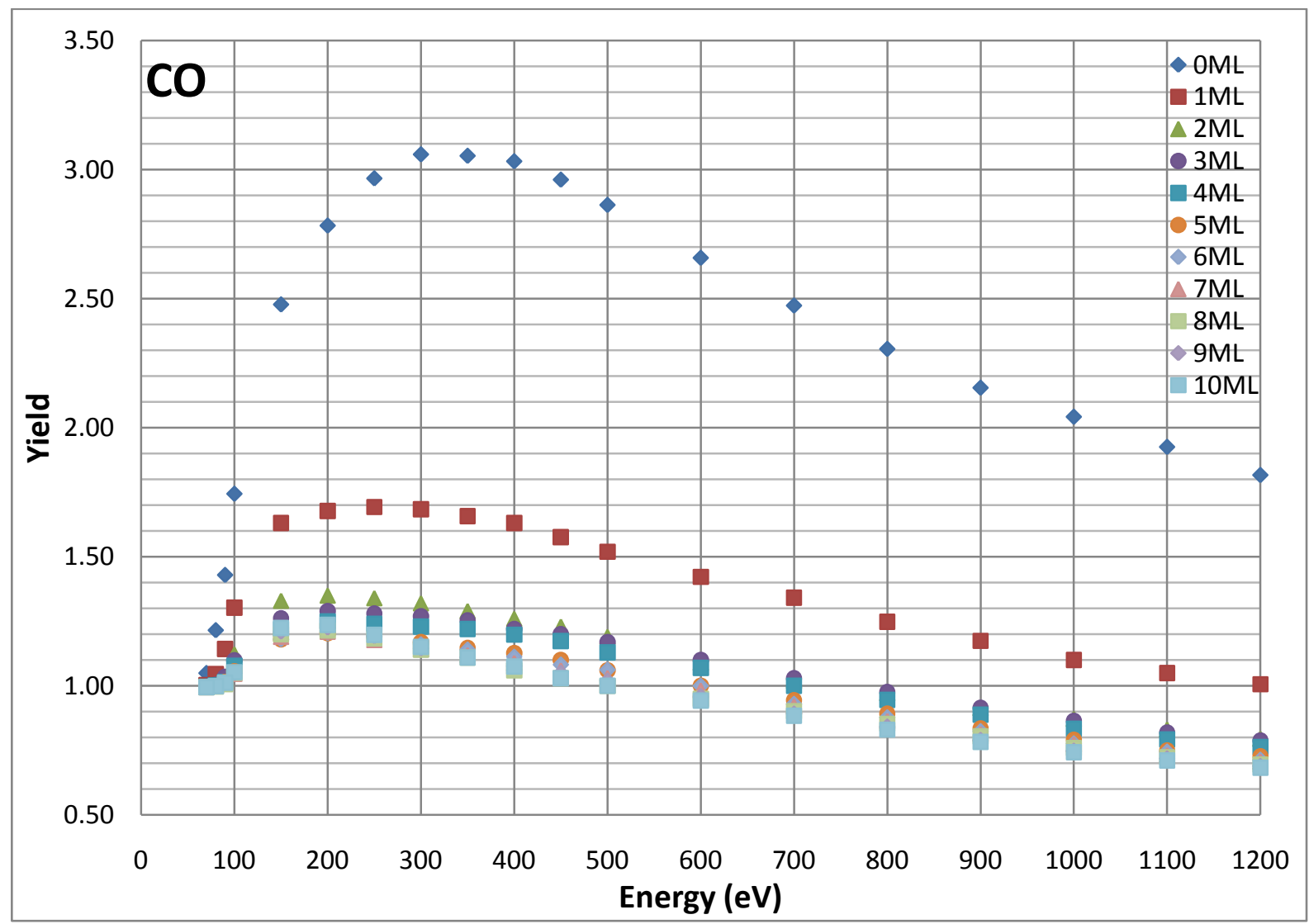

Figure 5.45: SEY of adsorbed CO as a function of energy for low coverage. 
Chapter 5-Results

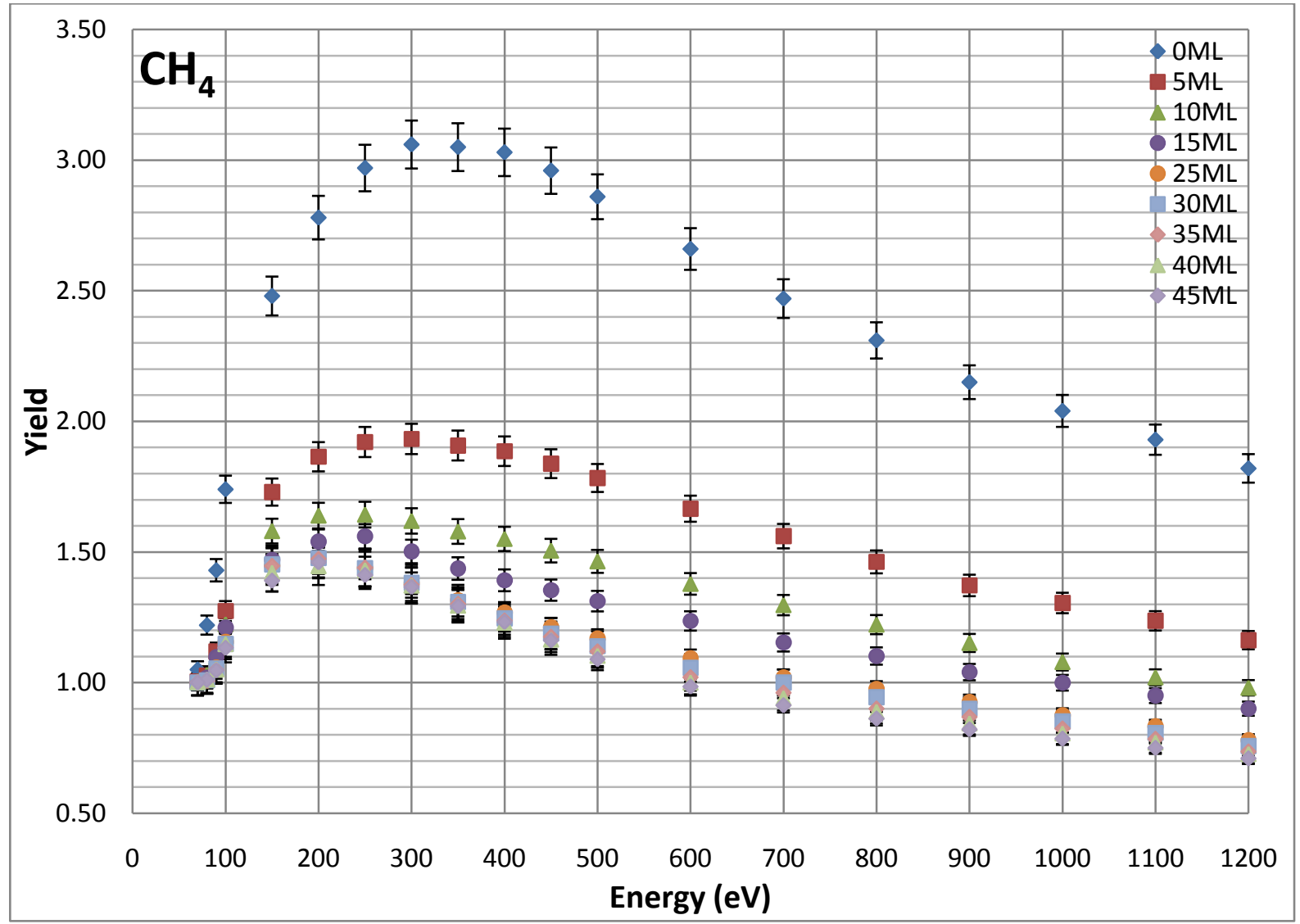

Figure 5.46: SEY of adsorbed $\mathrm{CH}_{4}$ as a function of energy for different coverages.

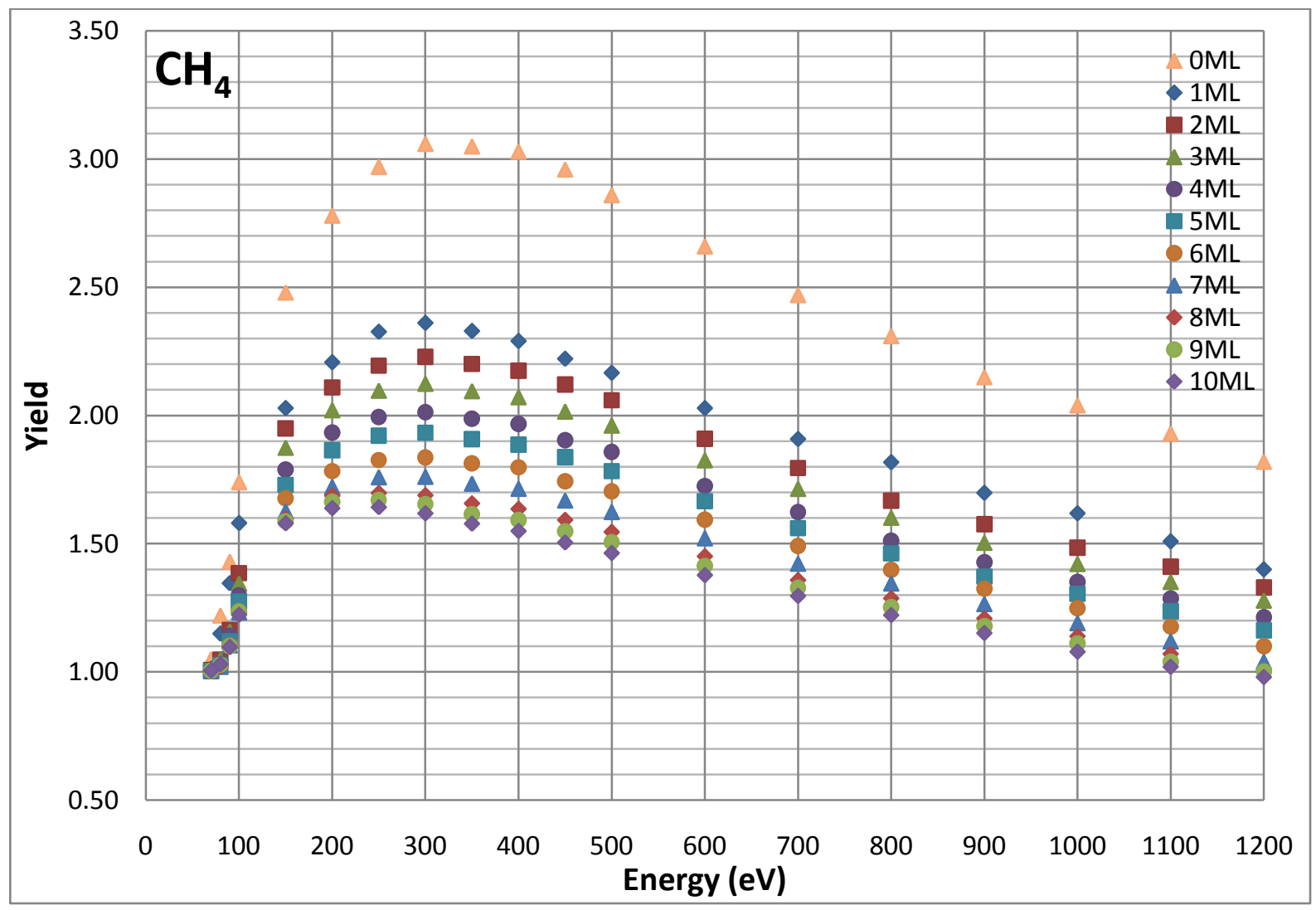

Figure 5.47: SEY of adsorbed $\mathrm{CH}_{4}$ as a function of energy for low coverage. 


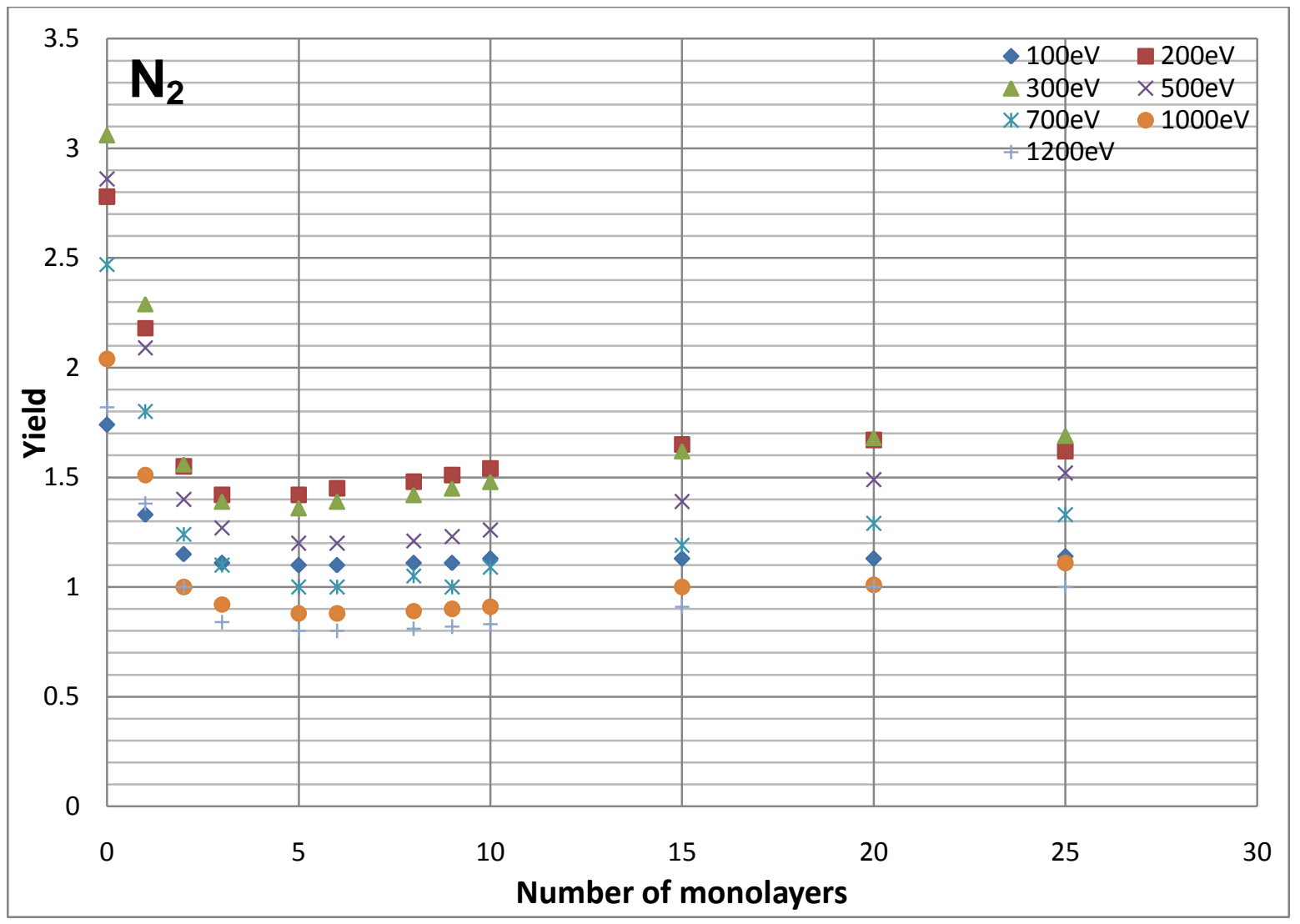

Figure 5.48: SEY of $\mathrm{N}_{2}$ as a function of coverage for defined primary electron energies.

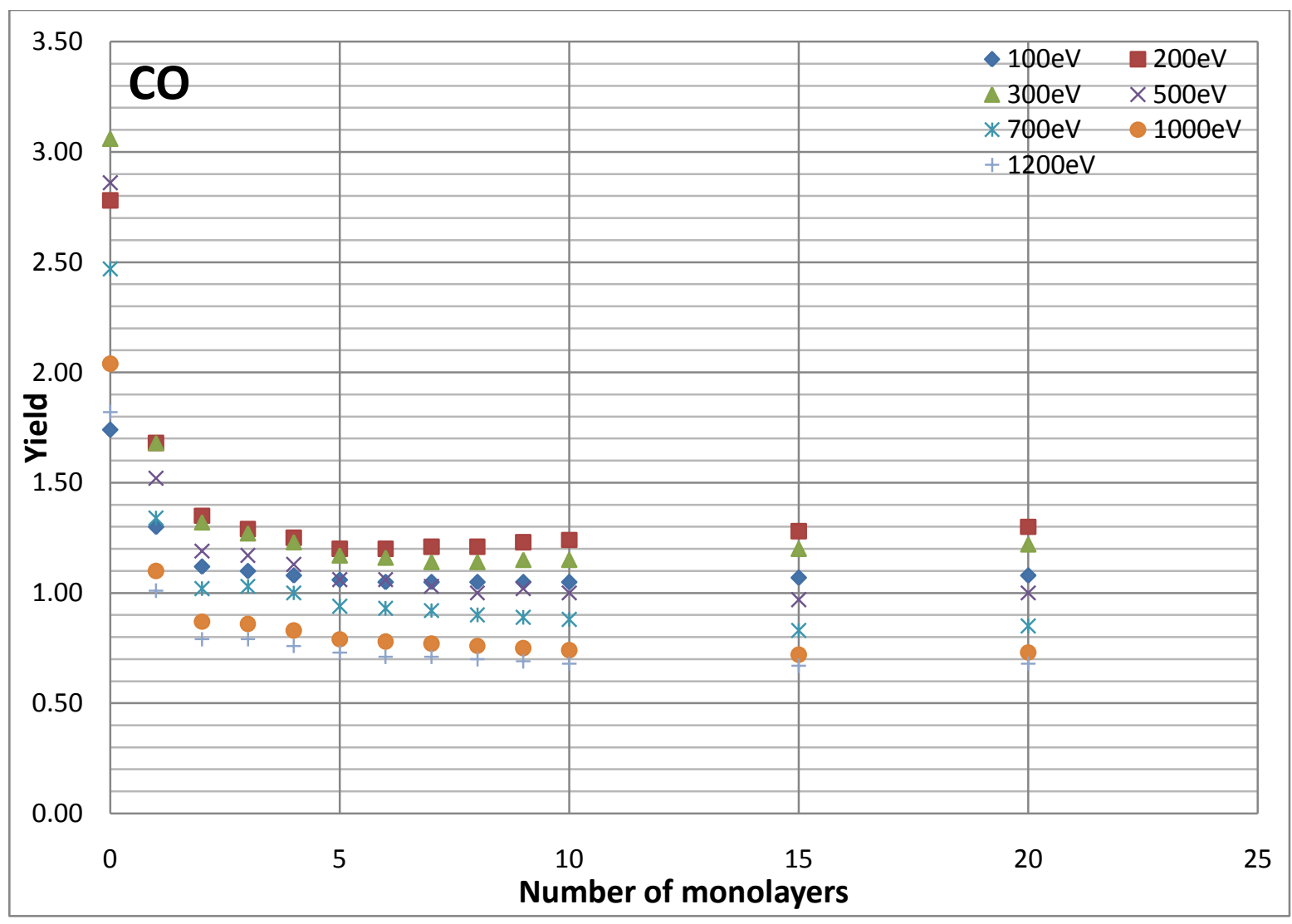

Figure 5.49: SEY of CO as a function of coverage for defined primary electron energies. 
Chapter 5-Results

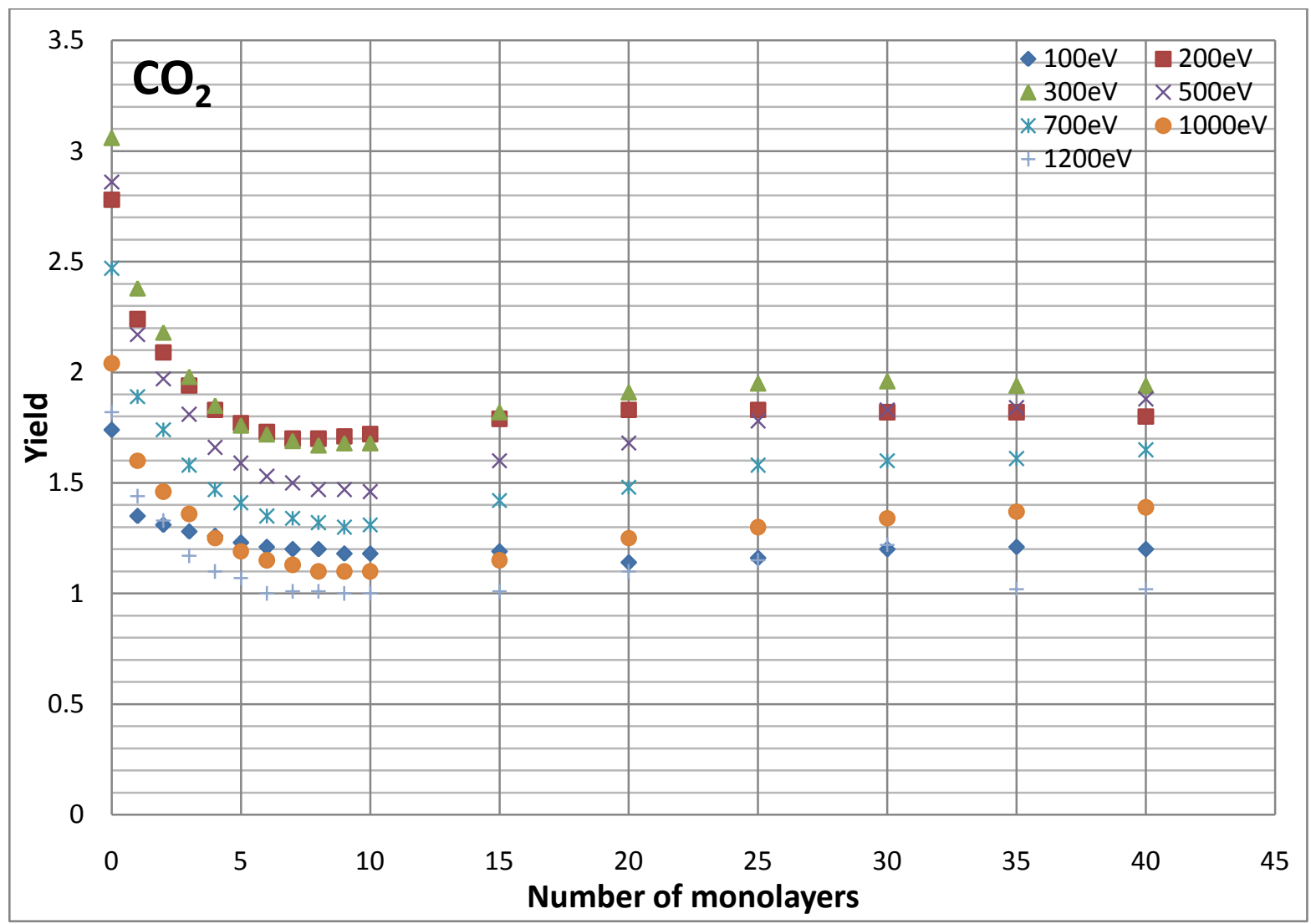

Figure 5.50: $\mathrm{SEY}$ of $\mathrm{CO}_{2}$ as a function of coverage for defined primary electron energies.

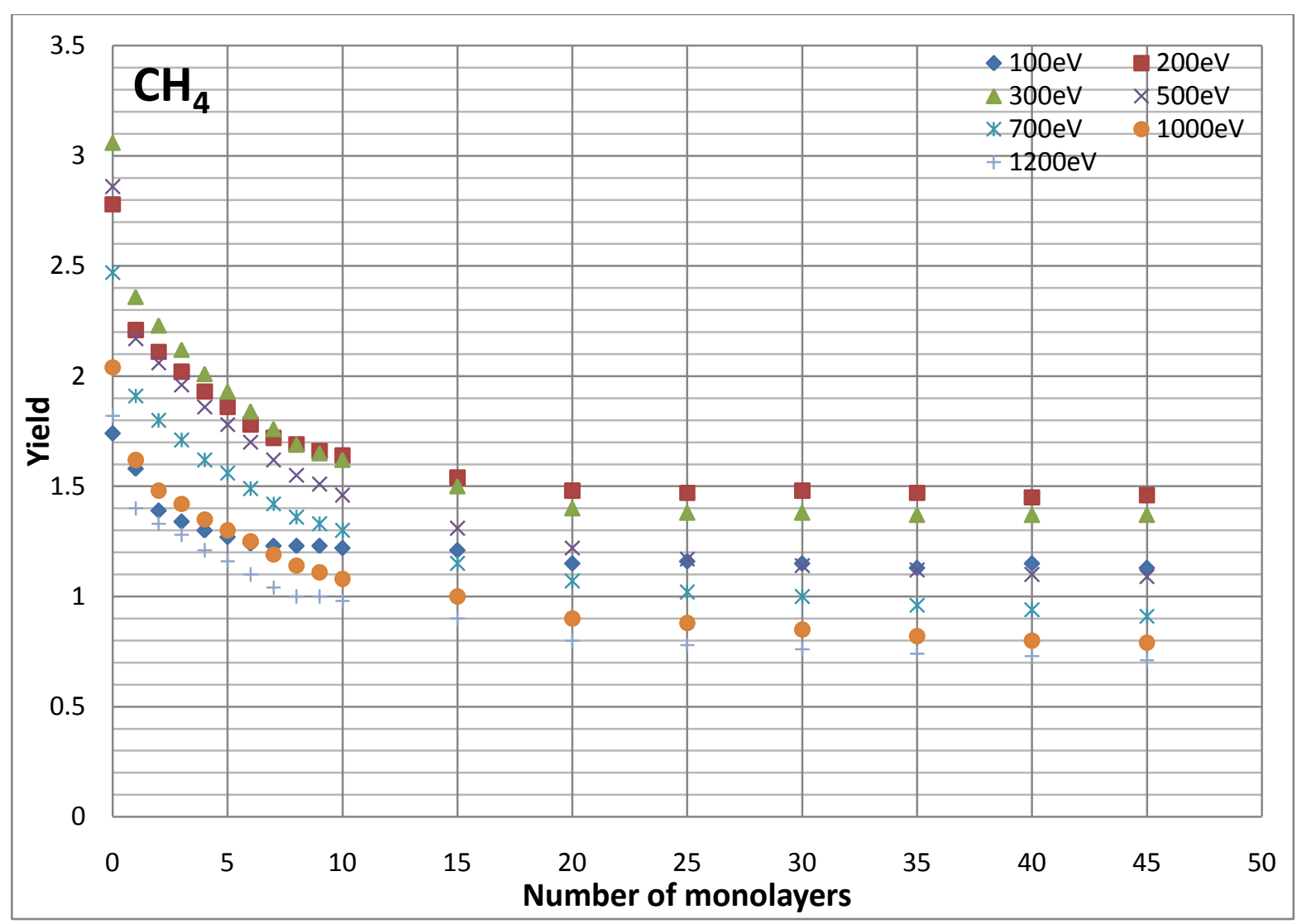

Figure 5.51: $\mathrm{SEY}$ of $\mathrm{CH}_{4}$ as a function of coverage for defined primary electron energies. 


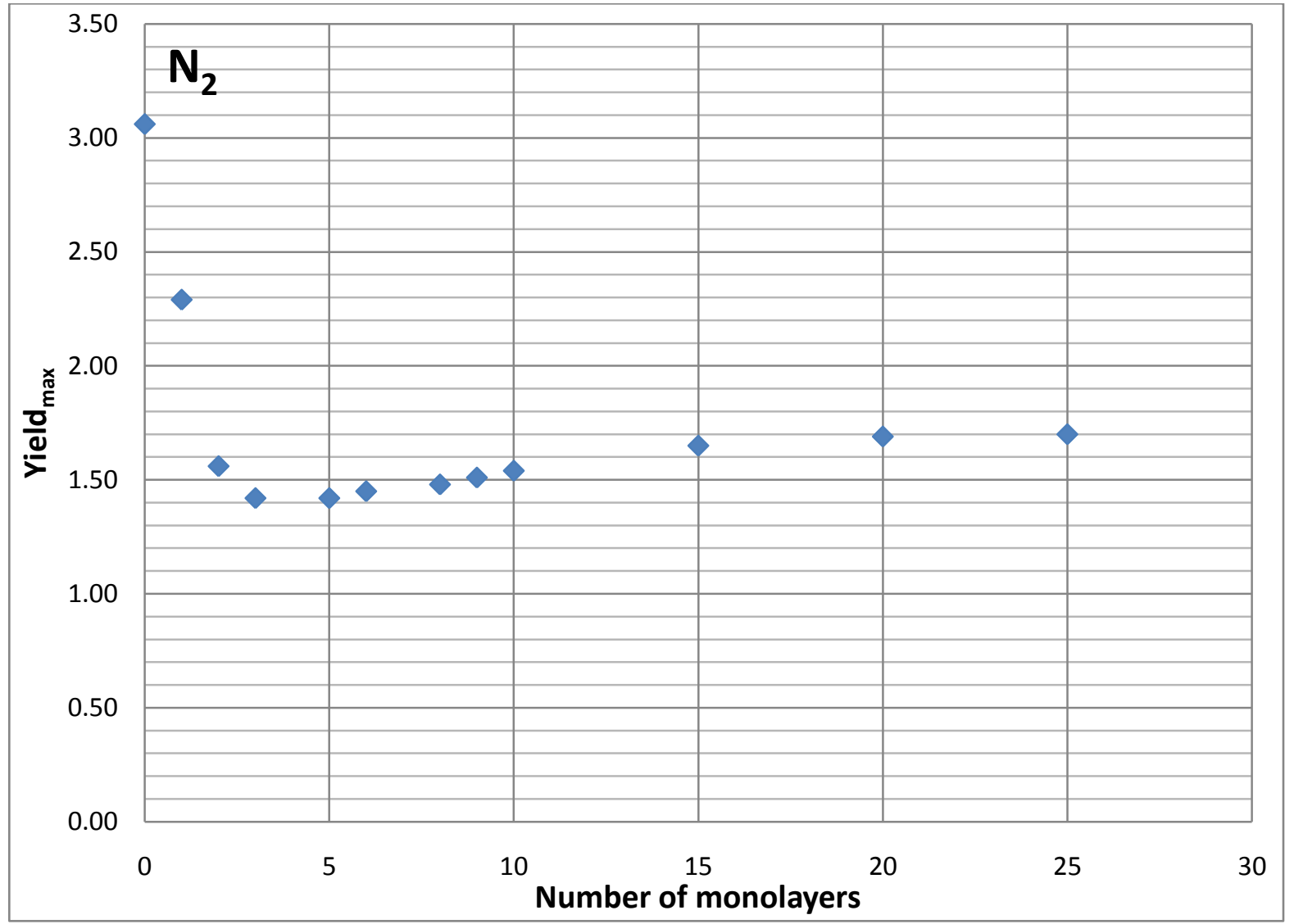

Figure 5.52: Maximum yield of $\mathrm{N}_{2}$ for each number of monolayer.

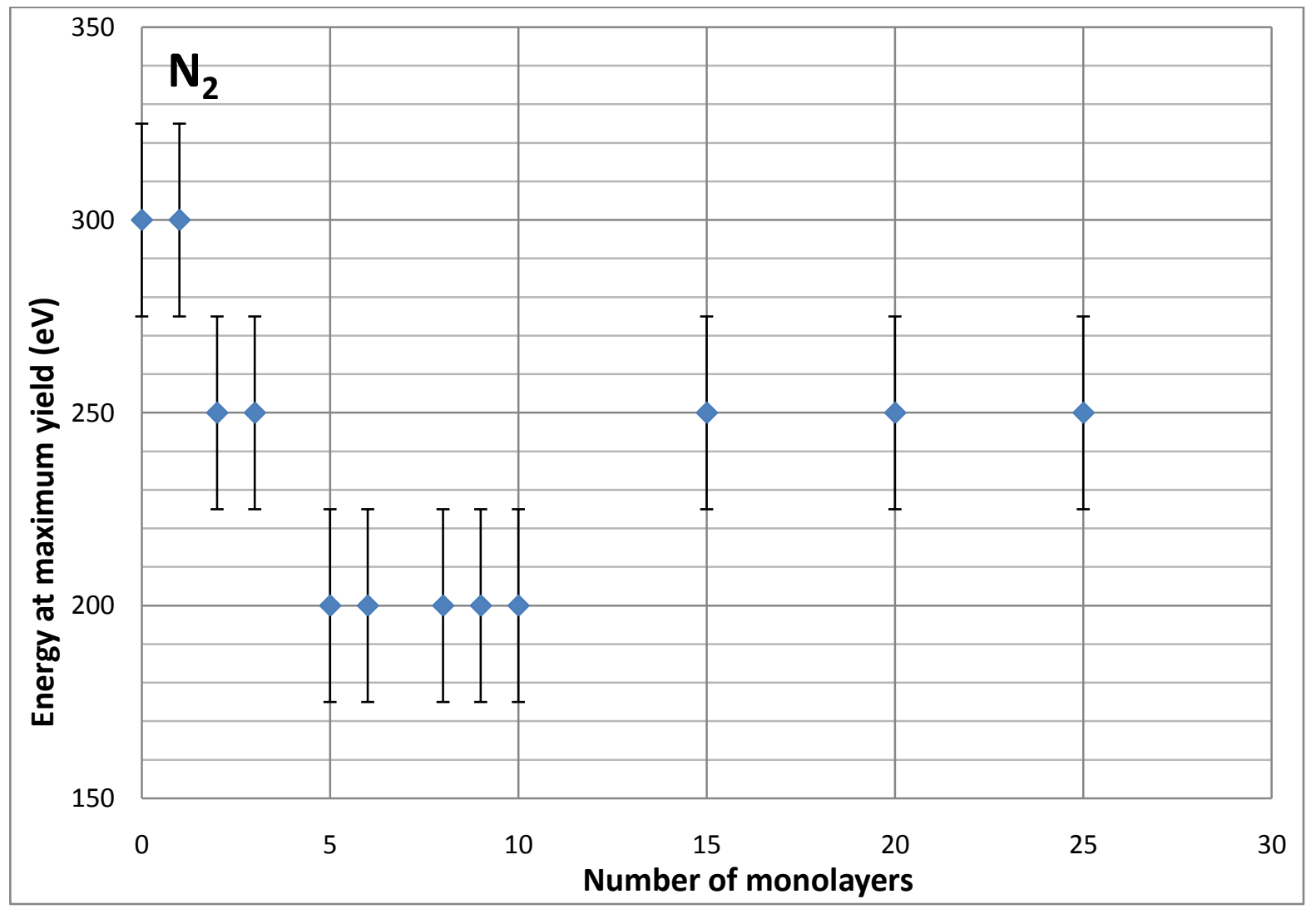

Figure 5.53: Energy at the maximum yield of $\mathrm{N}_{2}$ as a function of coverage. 


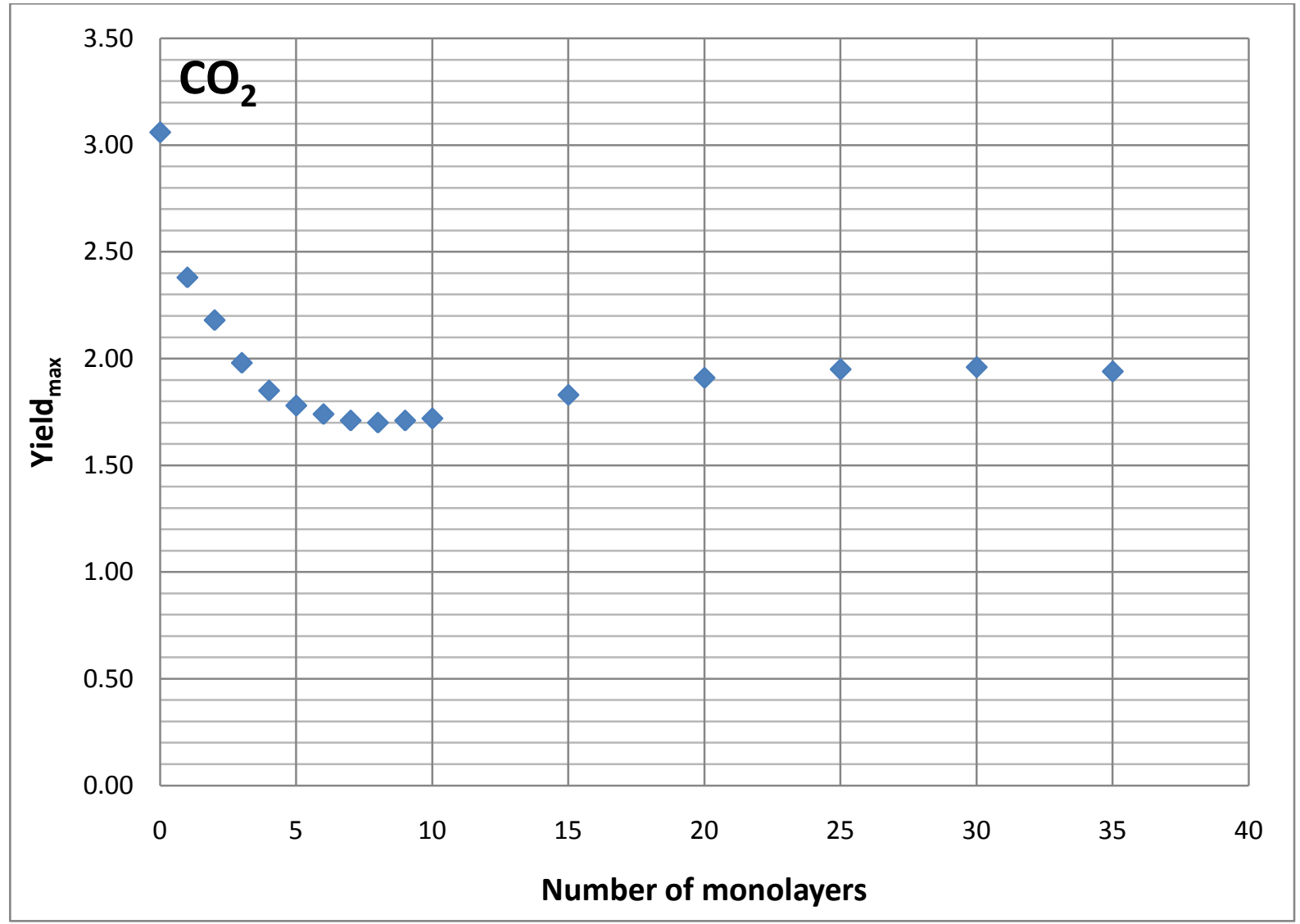

Figure 5.54: Maximum yield of $\mathrm{CO}_{2}$ for each number of monolayer.

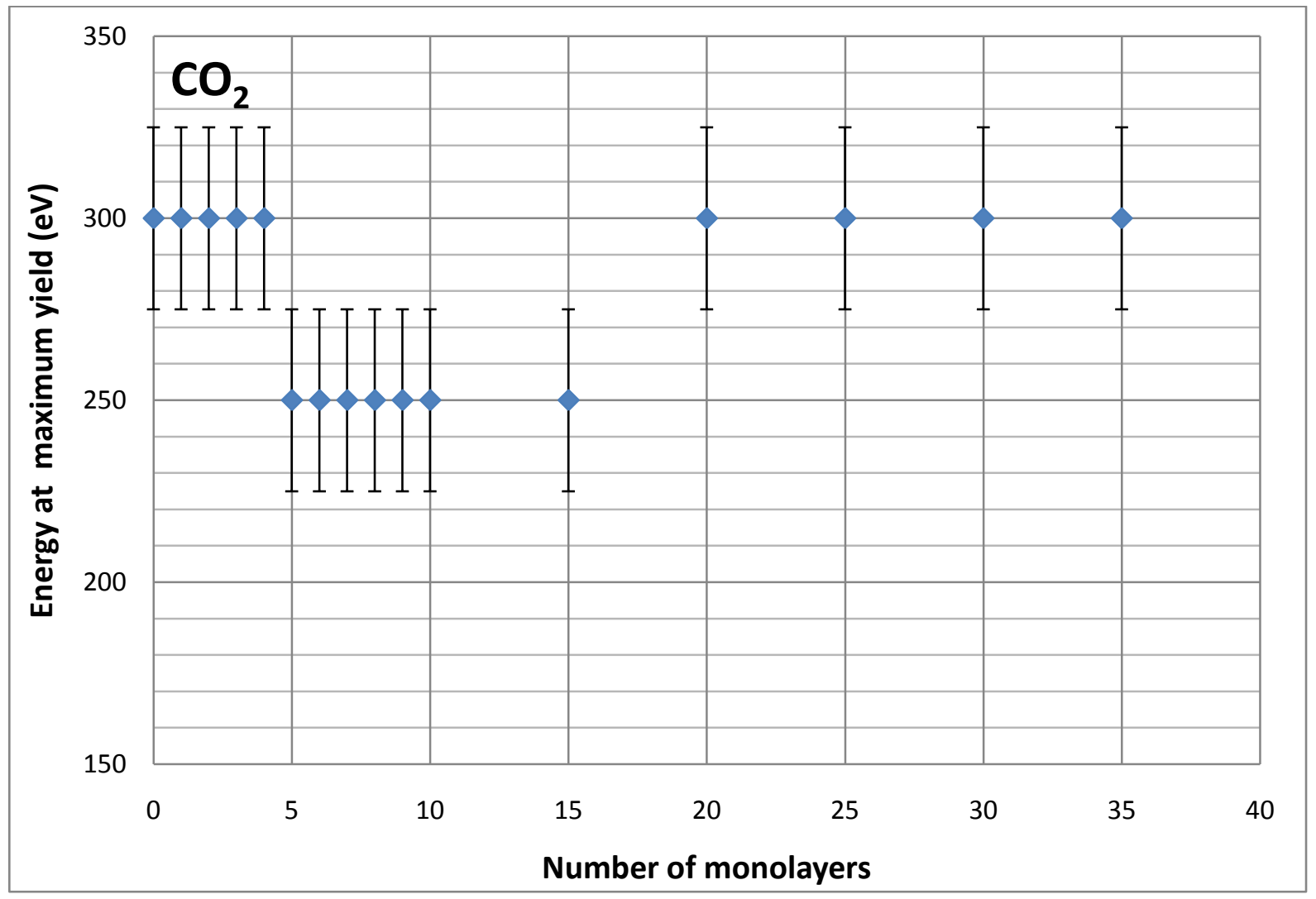

Figure 5.55: Energy at the maximum yield of $\mathrm{CO}_{2}$ as a function of coverage. 


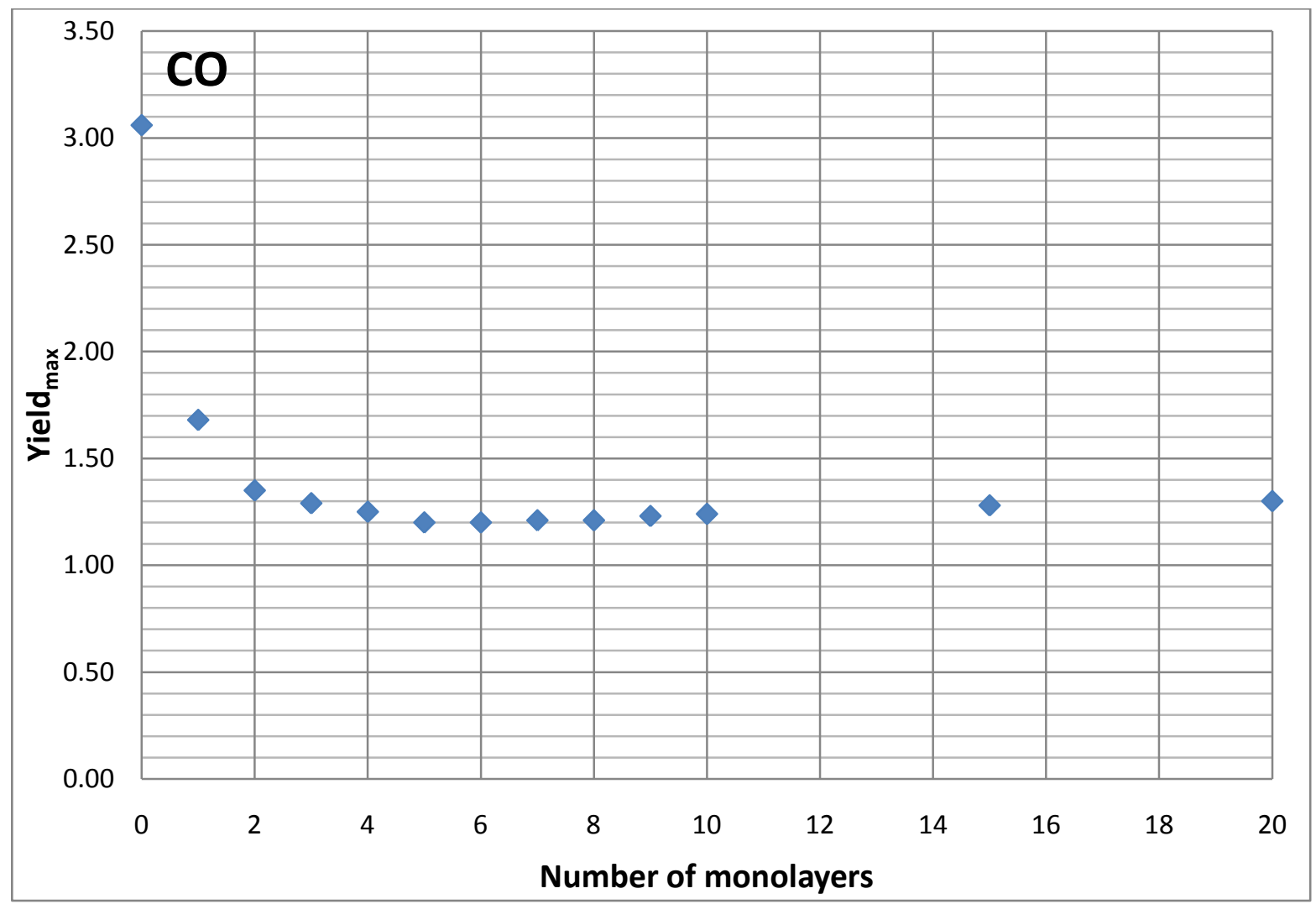

Figure 5.56: Maximum yield of CO for each number of monolayer.

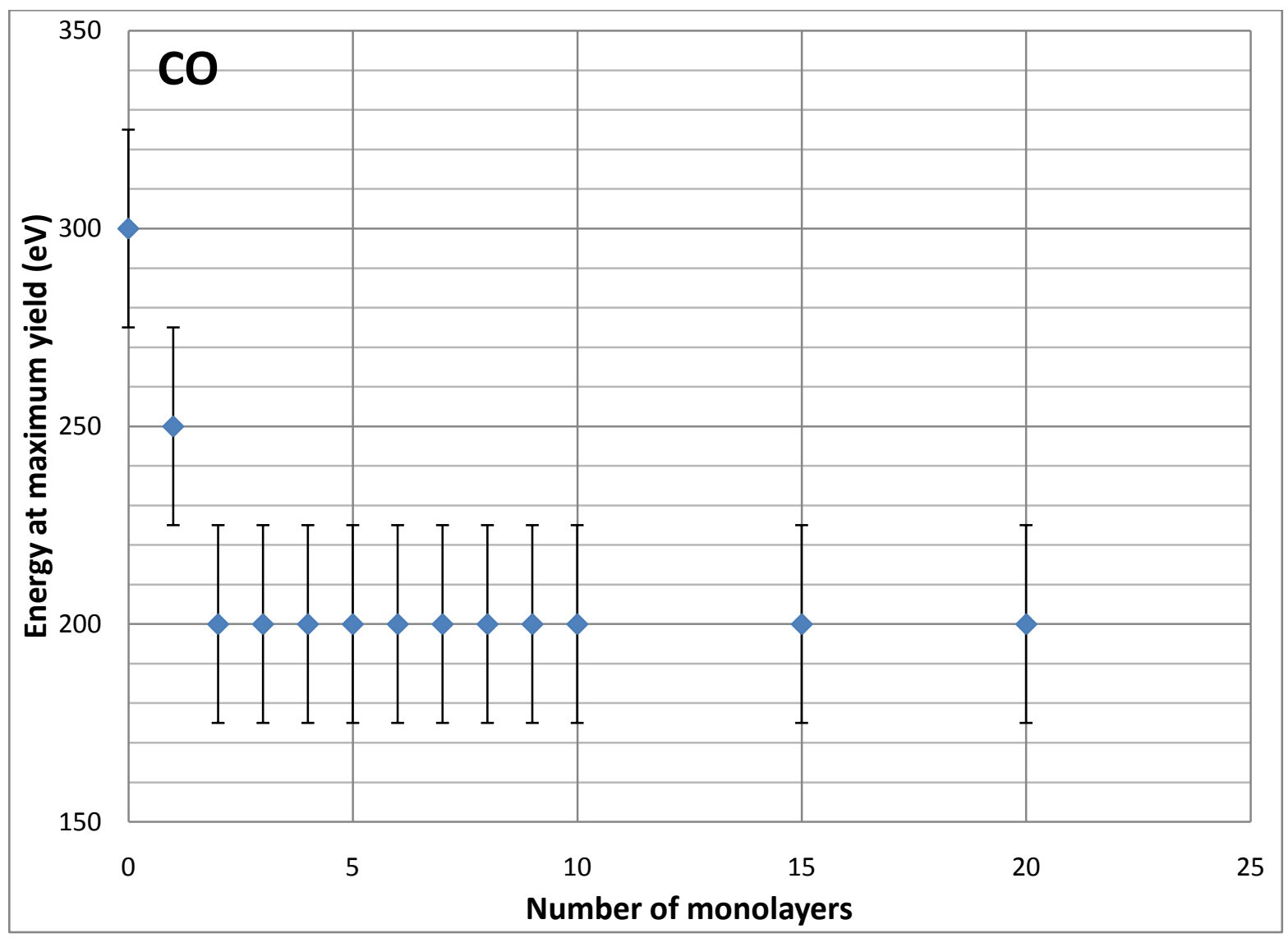

Figure 5.57: Energy at the maximum yield of $\mathrm{CO}$ as a function of coverage. 
Chapter 5-Results

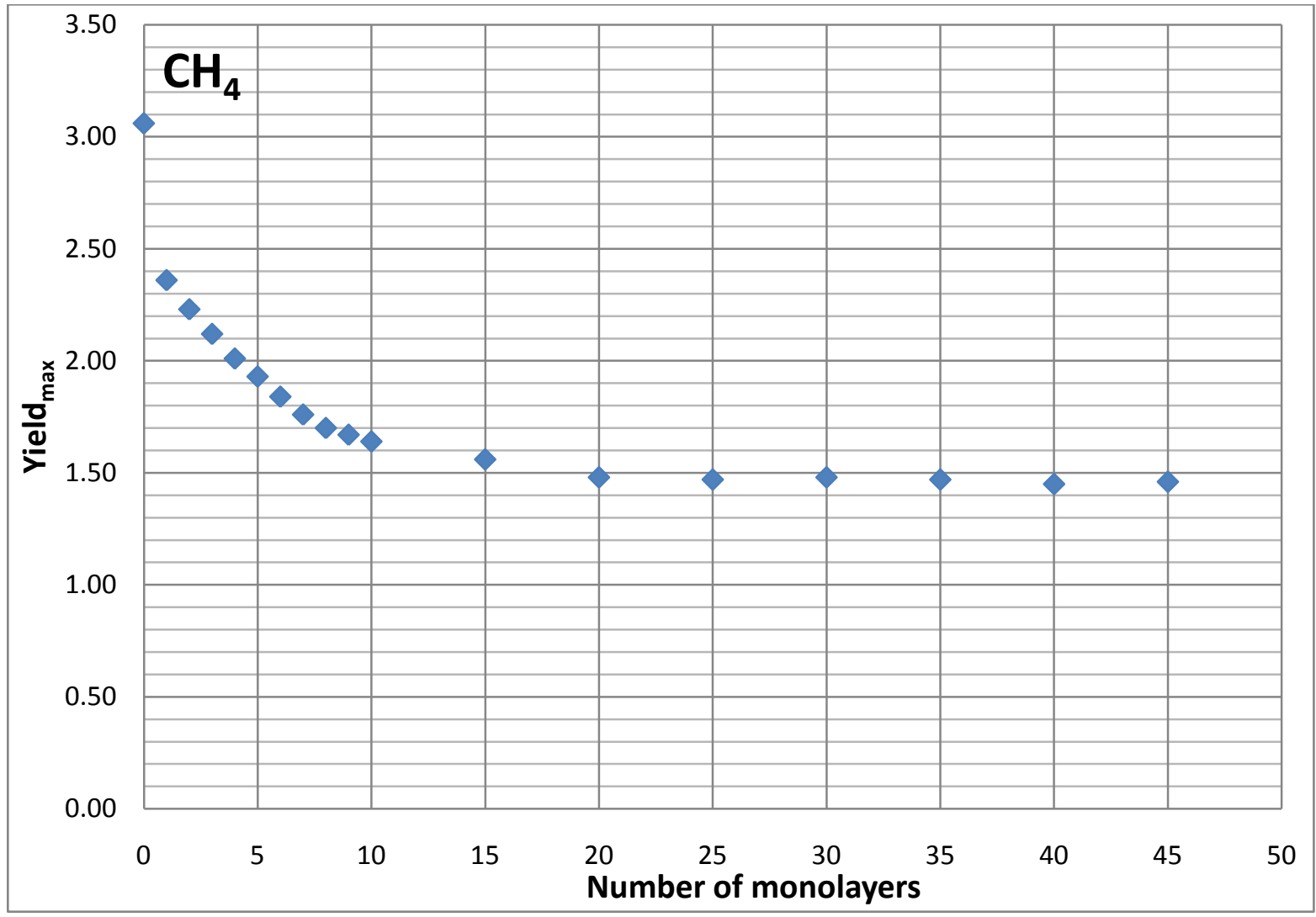

Figure 5.58: Maximum yield of $\mathrm{CH}_{4}$ for each number of monolayer.

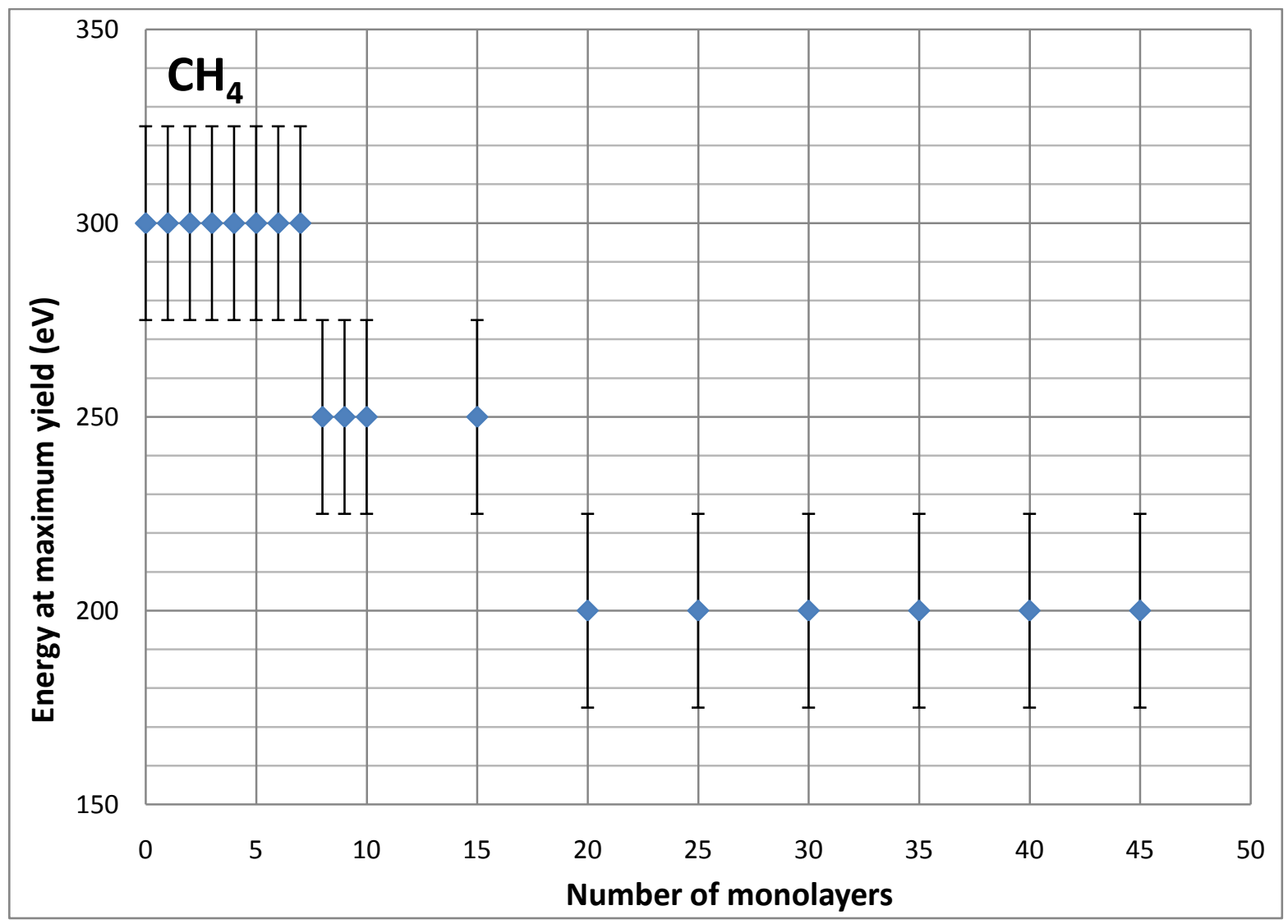

Figure 5.59: Energy at the maximum yield of $\mathrm{CH}_{4}$ as a function of coverage. 


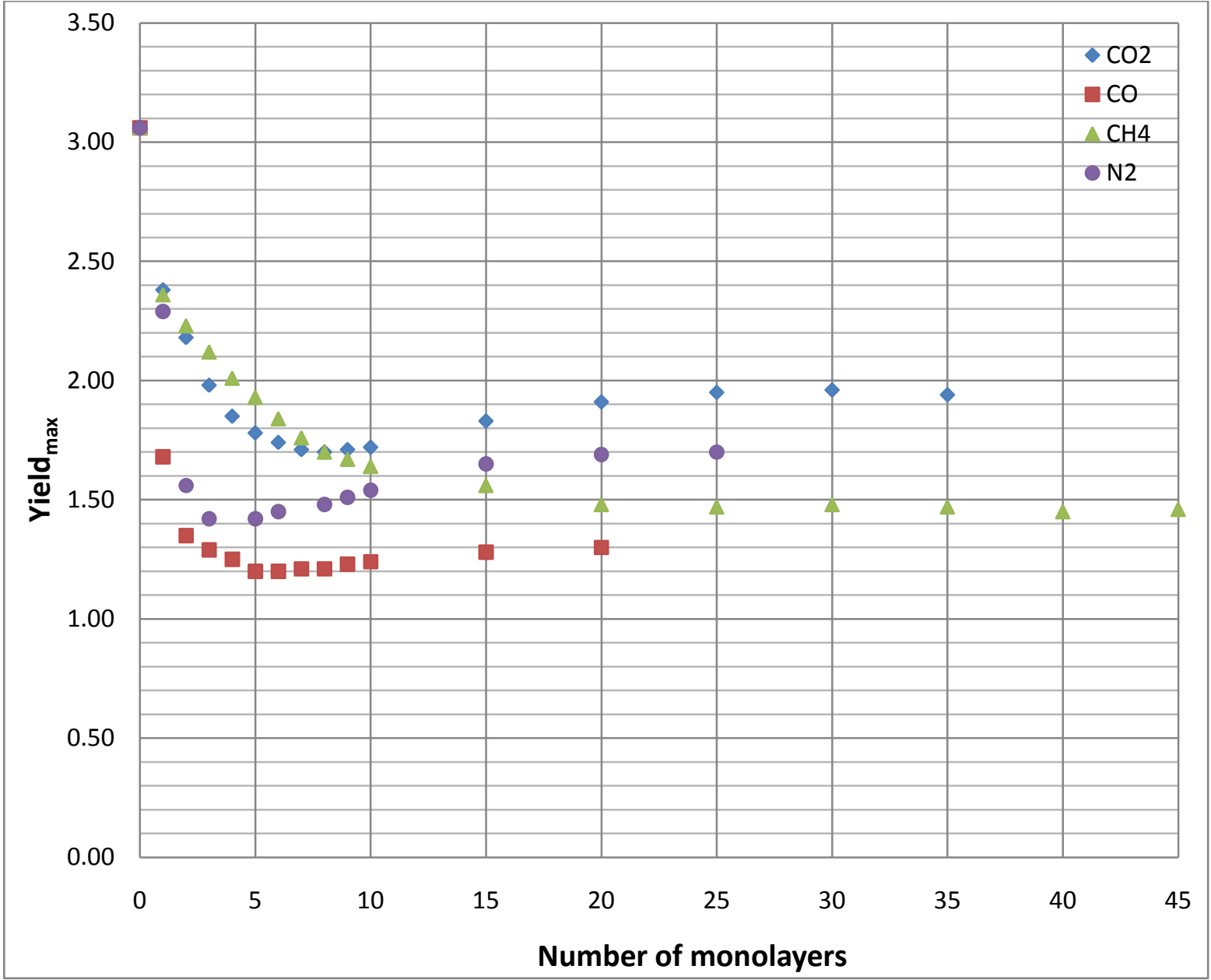

Figure 5.60: Maximum yield as a function of coverage for different adsorbates.

\subsubsection{SEY of electro polished copper}

The third substrate used for the measurement was electro polished copper. It has a lower secondary electron yield than copper due to the cleaner surface (Table 5.1). At room temperature the maximum yield is 1.73 and at cryogenic temperatures there is a slight difference and the yield is about 1.67. Figure 5.61 shows the yield at room temperature and at cryogenic temperatures.

\begin{tabular}{|l|l|l|}
\hline Parameters & Copper & $\begin{array}{l}\text { Electro polished } \\
\text { copper }\end{array}$ \\
\hline $\mathrm{R}_{\mathrm{z}}(\mu \mathrm{m})$ & $2.25 \pm 0.50$ & $0.68 \pm 0.08$ \\
\hline Surface Index & 1.0057 & 1.0004 \\
\hline
\end{tabular}

Table 5.1: Surface comparison of copper and electro polished copper. $R_{z}$ means "Maximum height of profile" and Surface index is the ratio of the surface area to the area of an ideal plane. 


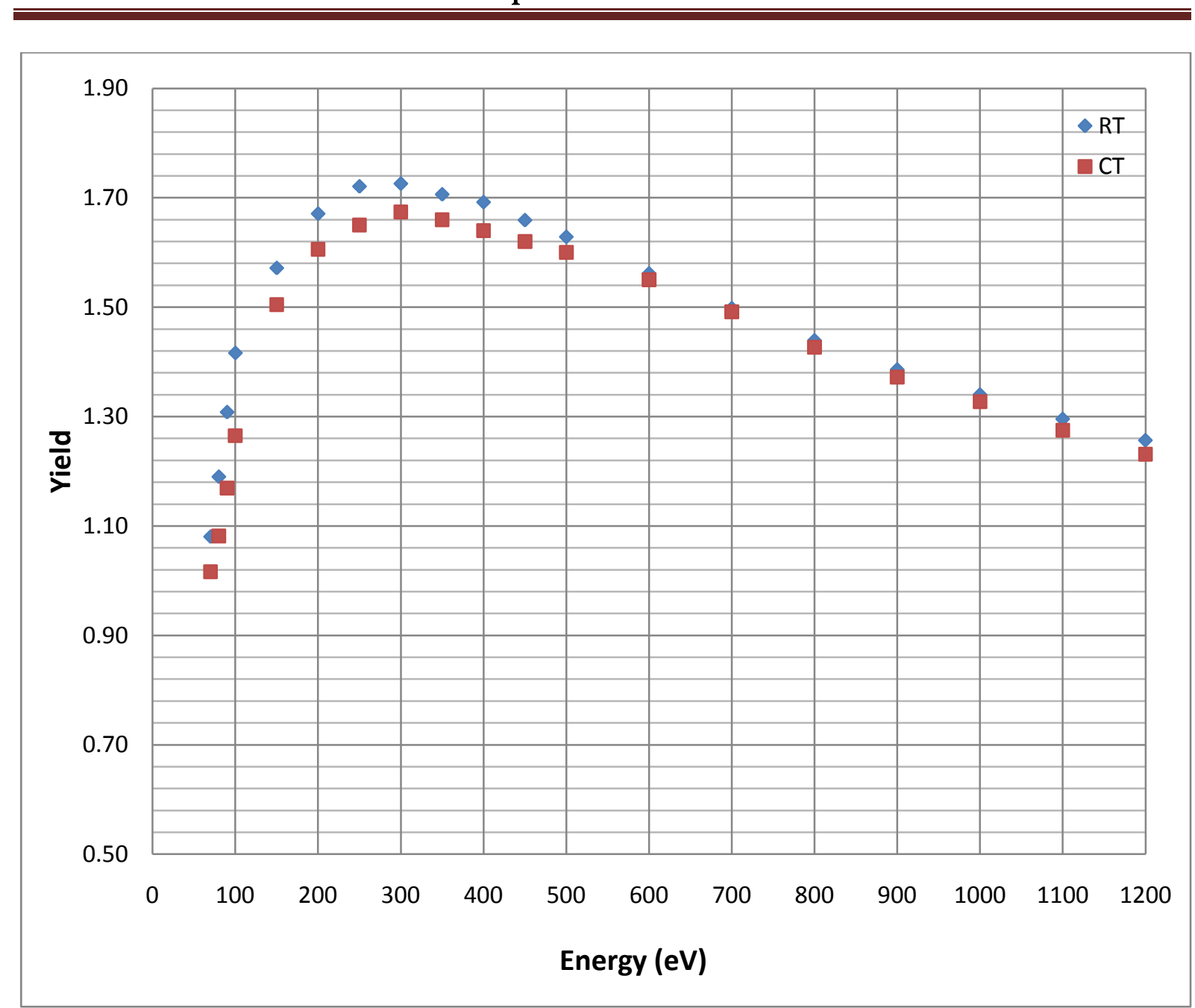

Figure 5.61: Secondary electron yield of electro polished copper at room temperature and at cryogenic temperatures. The yield is lower than the yield of copper due to the cleaner surface.

\subsubsection{SEY SEY of electro polished copper as a function of, $\mathrm{N}_{2}, \mathrm{CO}_{2}, \mathrm{CO}$ and $\mathrm{CH}_{4}$ adsorption}

The variation of the SEY as a function of 10 monolayers $\mathrm{N}_{2}, \mathrm{CO}_{2}, \mathrm{CO}$ and $\mathrm{CH}_{4}$ physisorbed on the electro polished copper is shown in Figure 5.62.

It is easy to see that the adsorption of 10 monolayers of carbon monoxide decreases the yield. In contrast, the adsorption of $\mathrm{N}_{2}, \mathrm{CO}_{2}$ and $\mathrm{CH}_{4}$ increases the yield. 


\section{Chapter 5-Results}

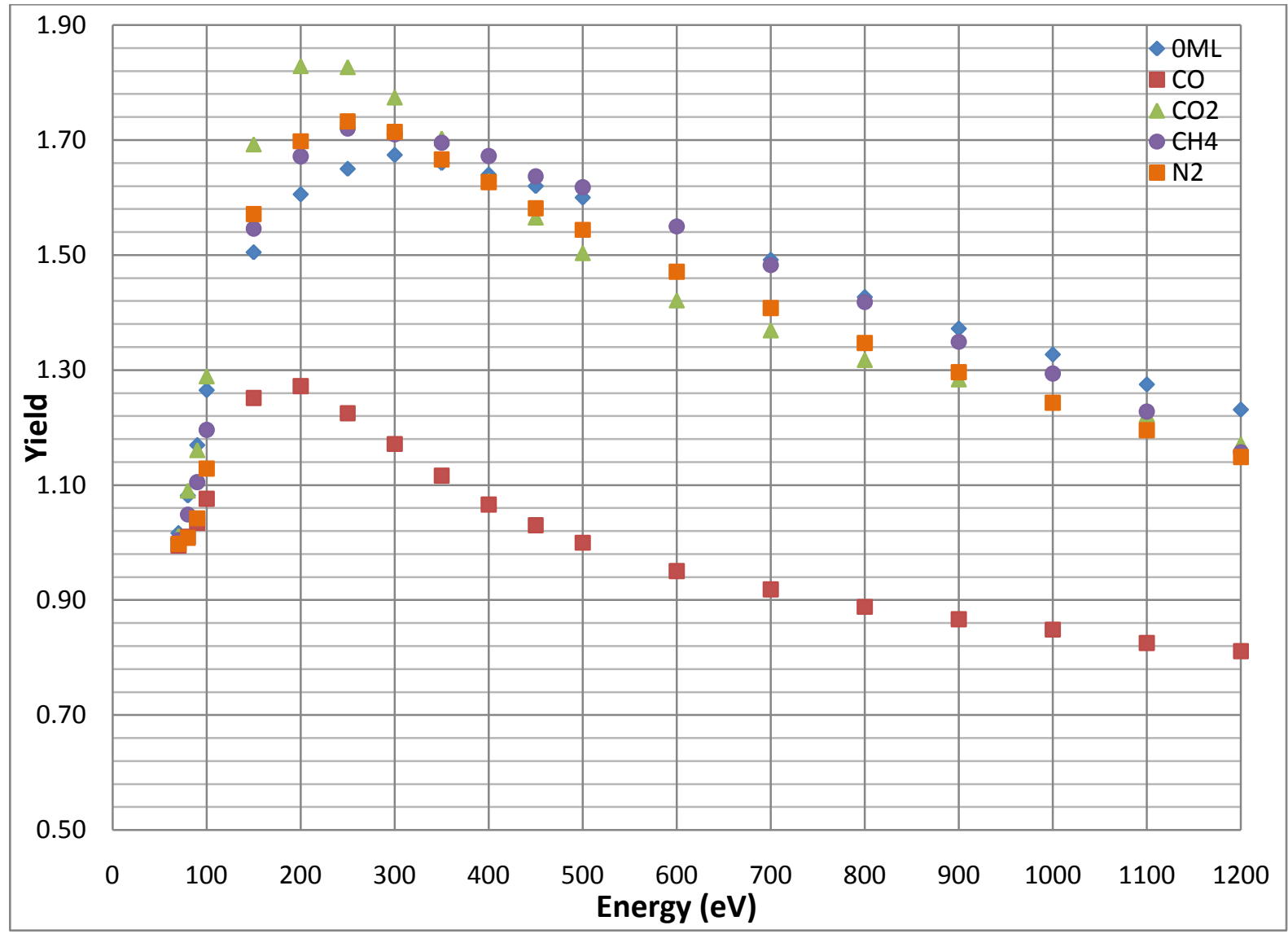

Figure 5.62: The secondary electron yield of 10 monolayers of $\mathrm{N}_{2}, \mathrm{CO}_{2}, \mathrm{CO}$ and $\mathrm{CH}_{4}$ physisorbed on electro polished copper.

The energy for maximum yield is different for different gases. While $\mathrm{CO}_{2}, \mathrm{~N}_{2}$ and $\mathrm{CH}_{4}$ have the maximum yield at $250 \mathrm{eV}, \mathrm{CO}$ has it at $200 \mathrm{eV}$. Electro-polished copper has the maximum yield at $300 \mathrm{eV}$. The secondary electron yield for each gas with a different number of monolayers as a function of the primary electron energy is shown in figures 5.63 to 5.70 . For each gas the first curves show the SEY for high coverage and the second curves show the SEY with low coverage.

The figure $5.71,5.72,5.73$, and 5.74 shows the secondary electron yield as a function of the coverage for some selected primary electron energies.

The maximum yield at different numbers of coverage for each gas and the primary electron energy at the maximum yield as a function of coverage are shown in figure 5.75 to 5.82 .

In the last figure (Figure 5.83) the maximum yield of all the gases used for the measurements are shown in one single curve as a function of coverage. 
Chapter 5-Results

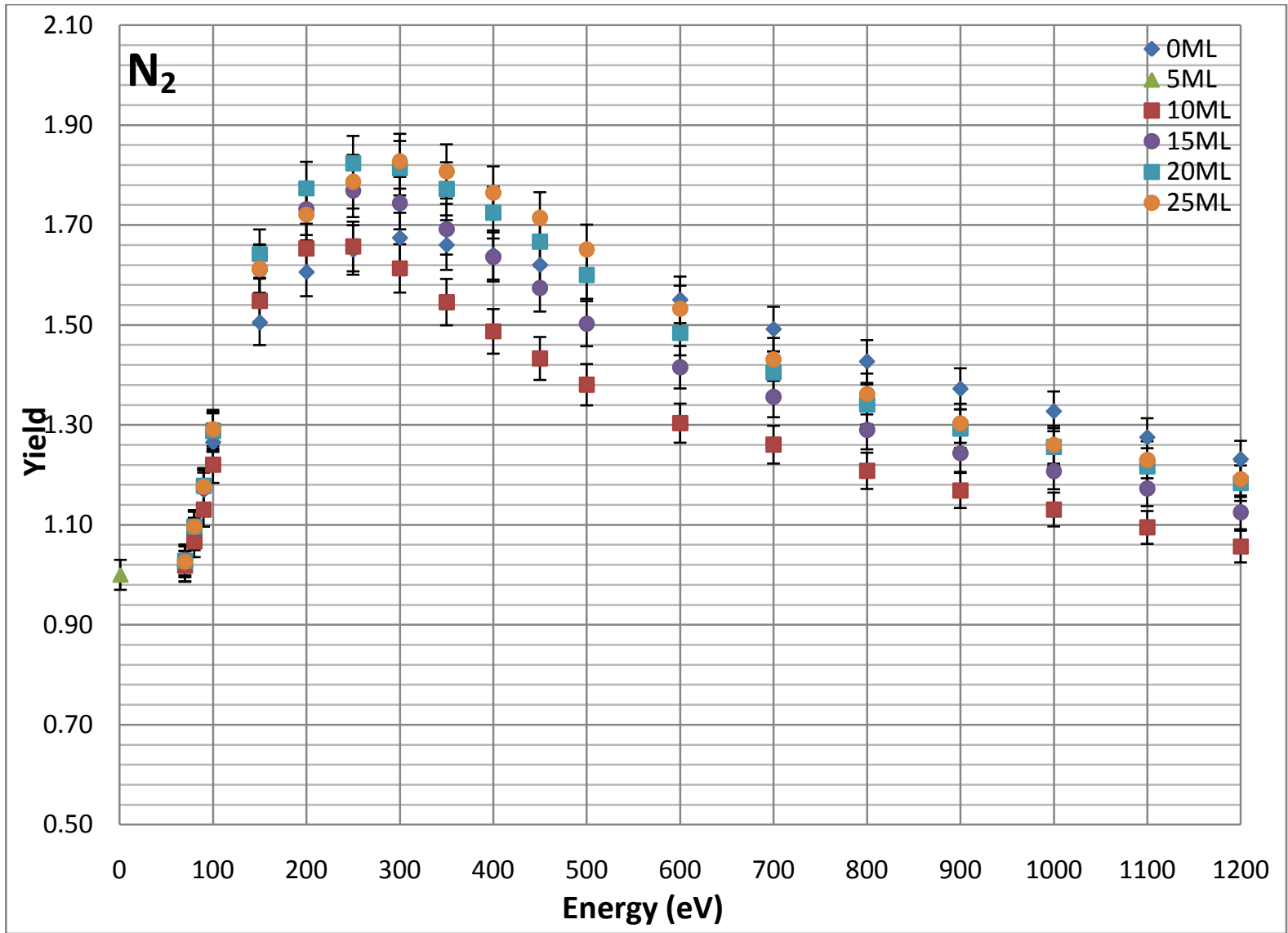

Figure 5.63: SEY of adsorbed $\mathrm{N}_{2}$ as a function of energy for different coverages.

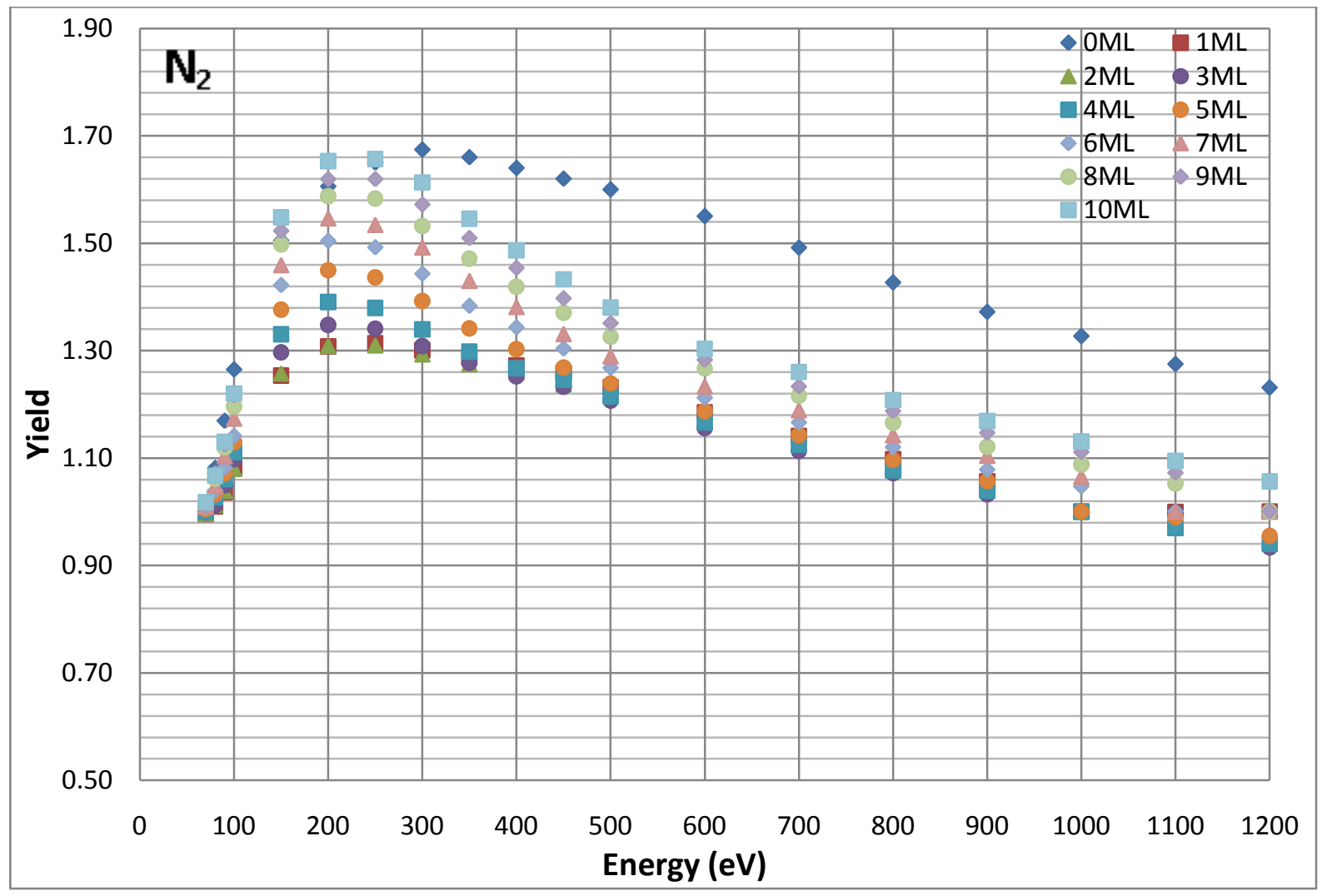

Figure 5.64: SEY of adsorbed $\mathrm{N}_{2}$ as a function of energy for low coverage. 
Chapter 5-Results

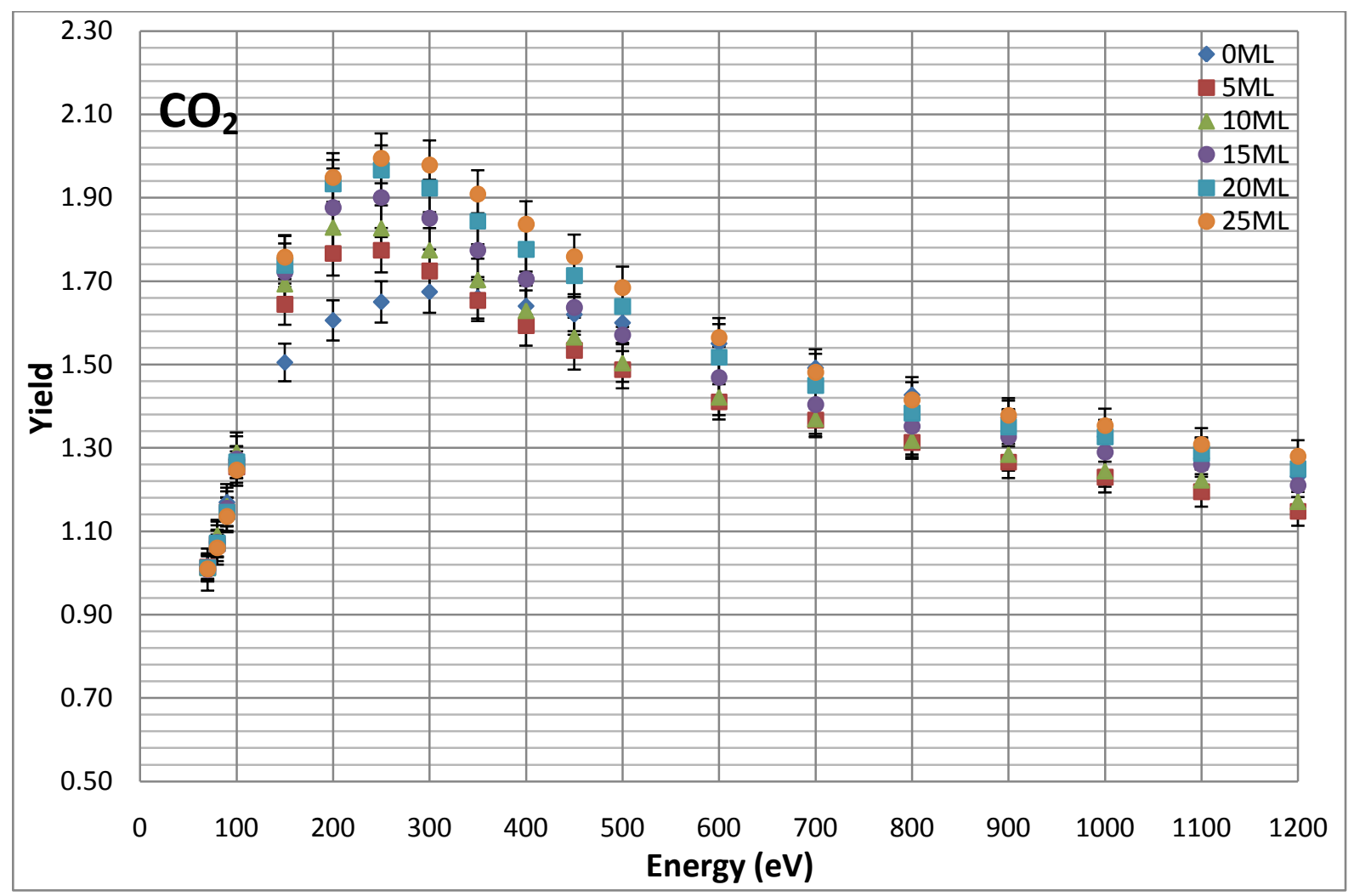

Figure 5.65: SEY of adsorbed $\mathrm{CO}_{2}$ as a function of energy for different coverages.

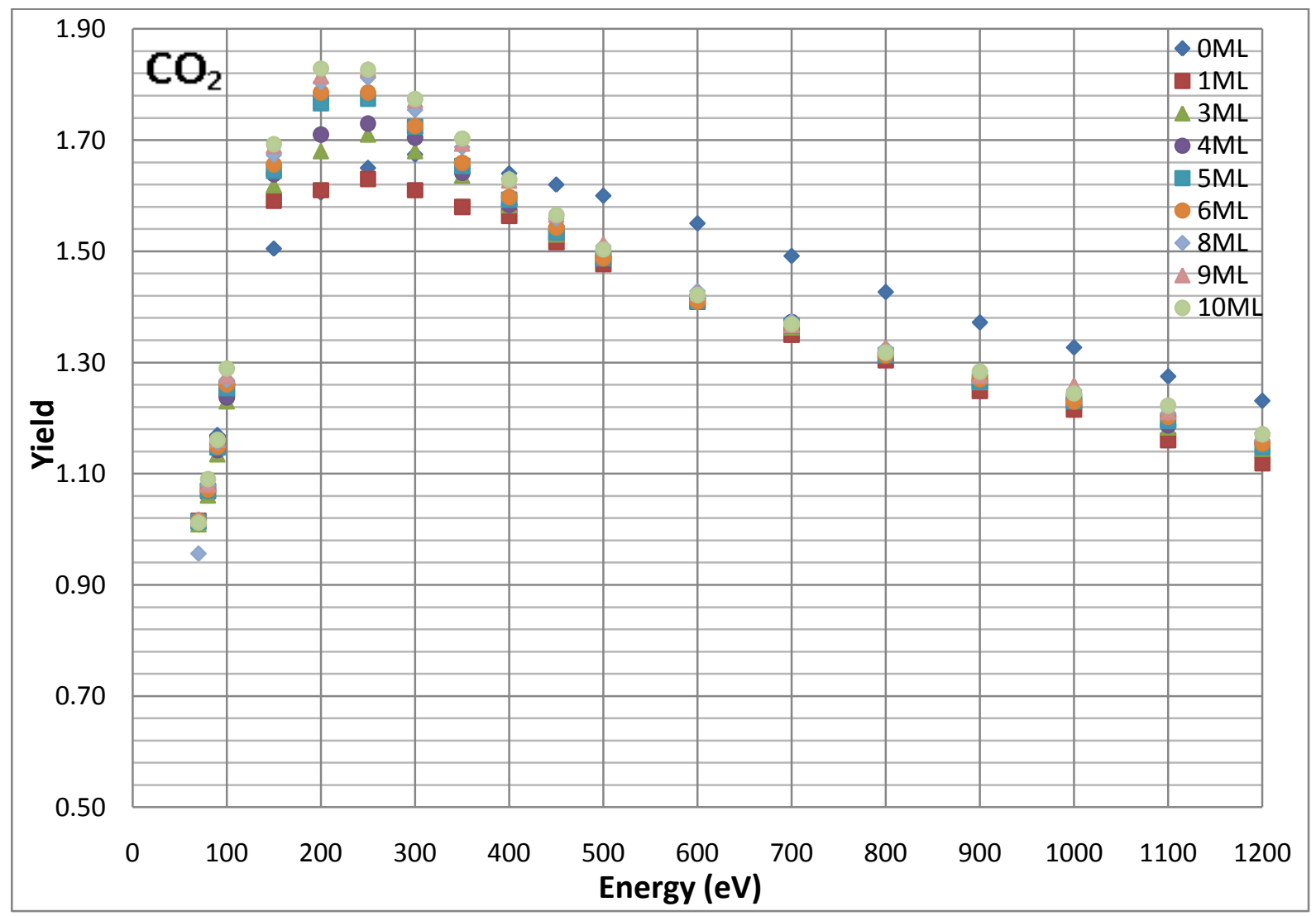

Figure 5.66: SEY of adsorbed $\mathrm{CO}_{2}$ as a function of energy for low coverage. 
Chapter 5-Results

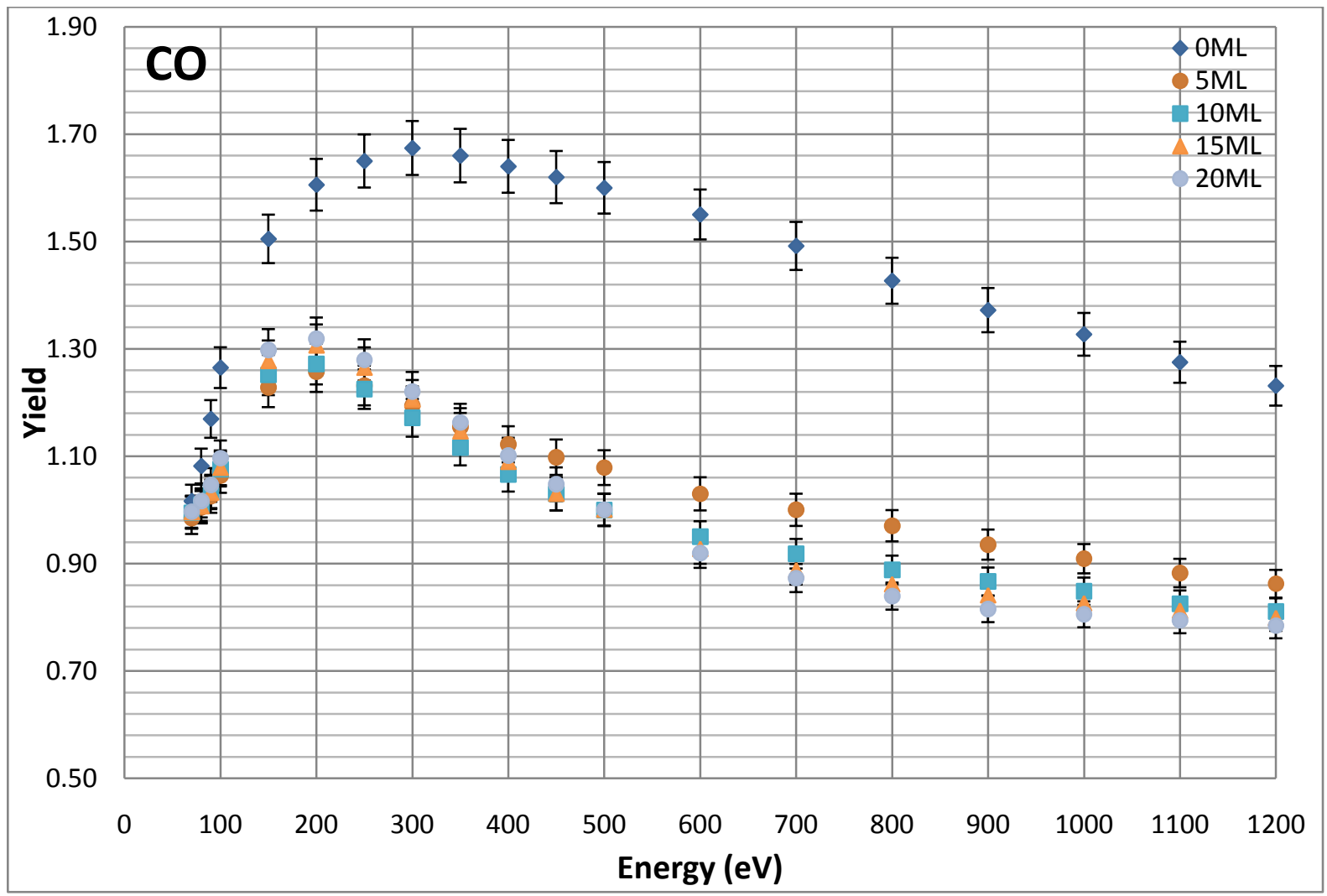

Figure 5.67: SEY of adsorbed CO as a function of energy for different coverages.

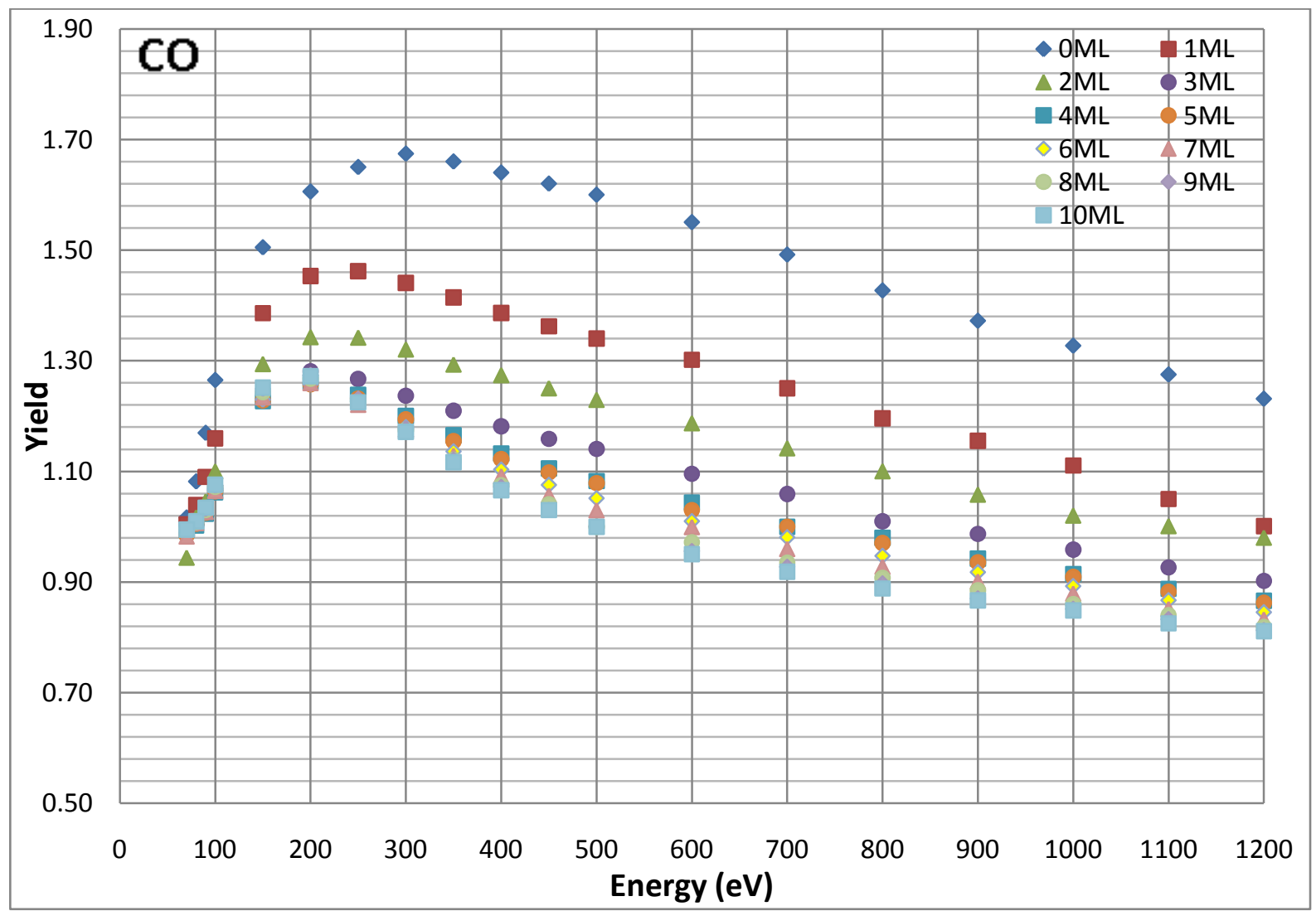

Figure 5.68: SEY of adsorbed CO as a function of energy for low coverage. 


\section{Chapter 5-Results}

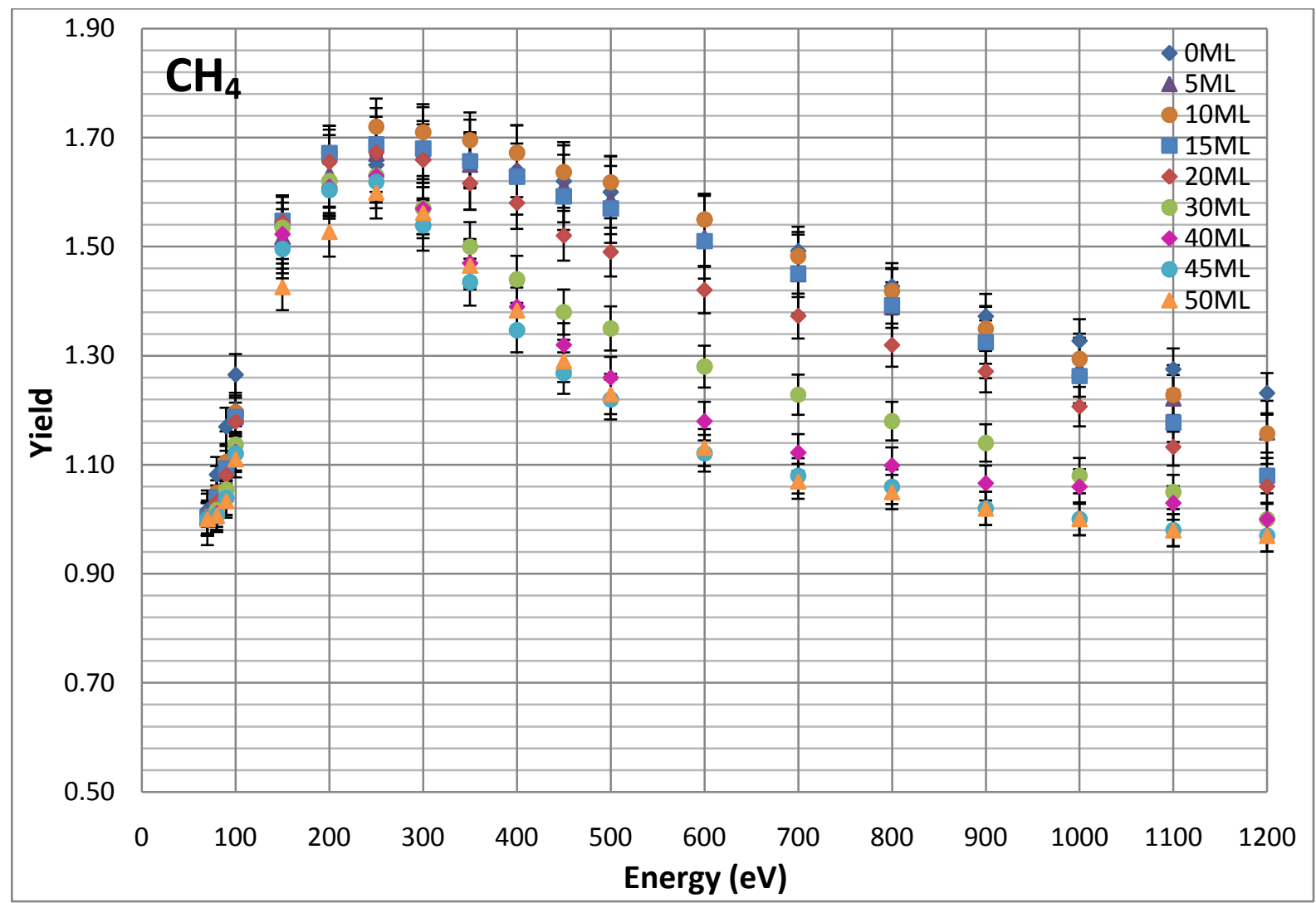

Figure 5.69: $\mathrm{SEY}$ of adsorbed $\mathrm{CH}_{4}$ as a function of energy for different coverages.

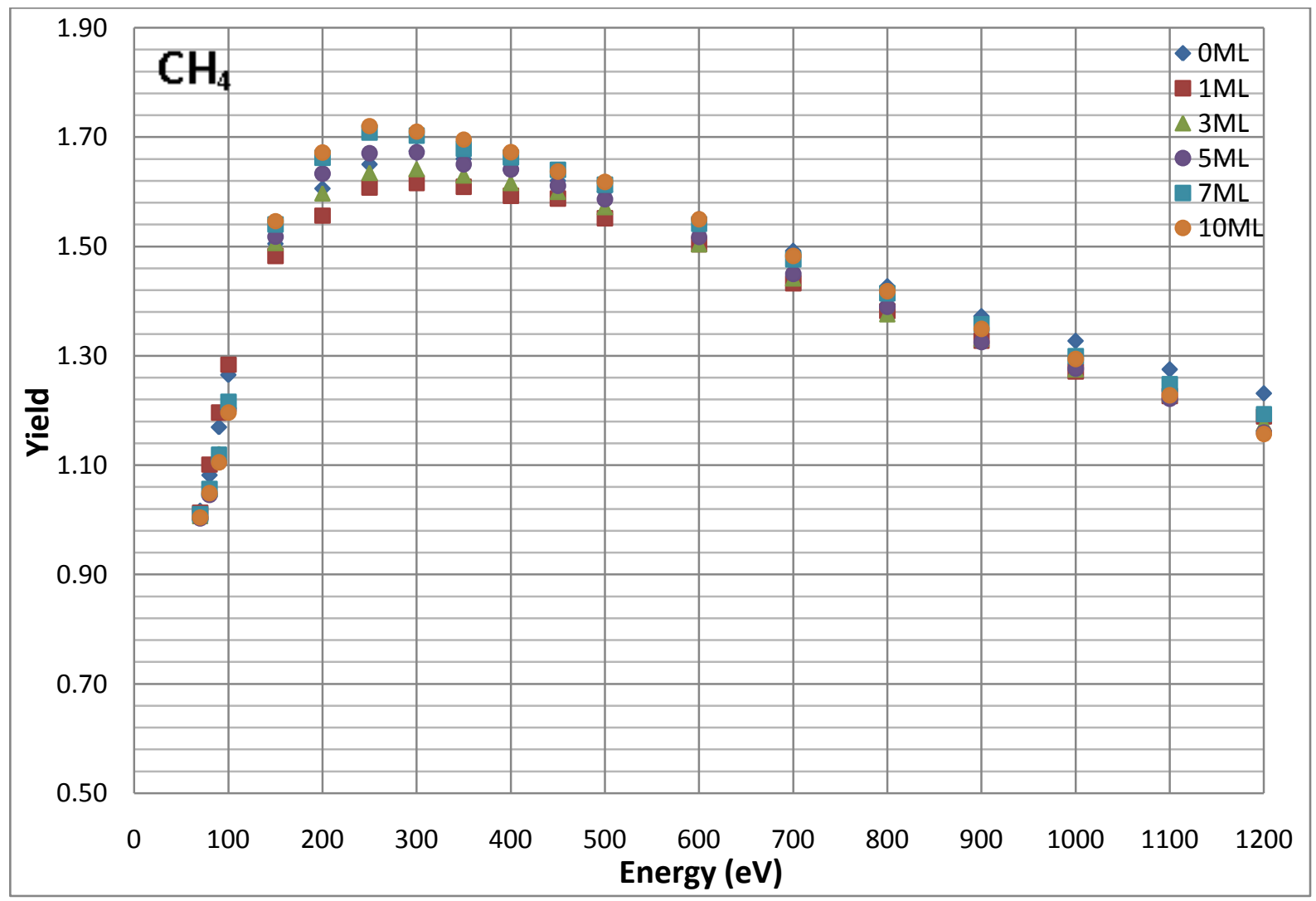

Figure 5.70: $\mathrm{SEY}$ of adsorbed $\mathrm{CH}_{4}$ as a function of energy for low coverage. 


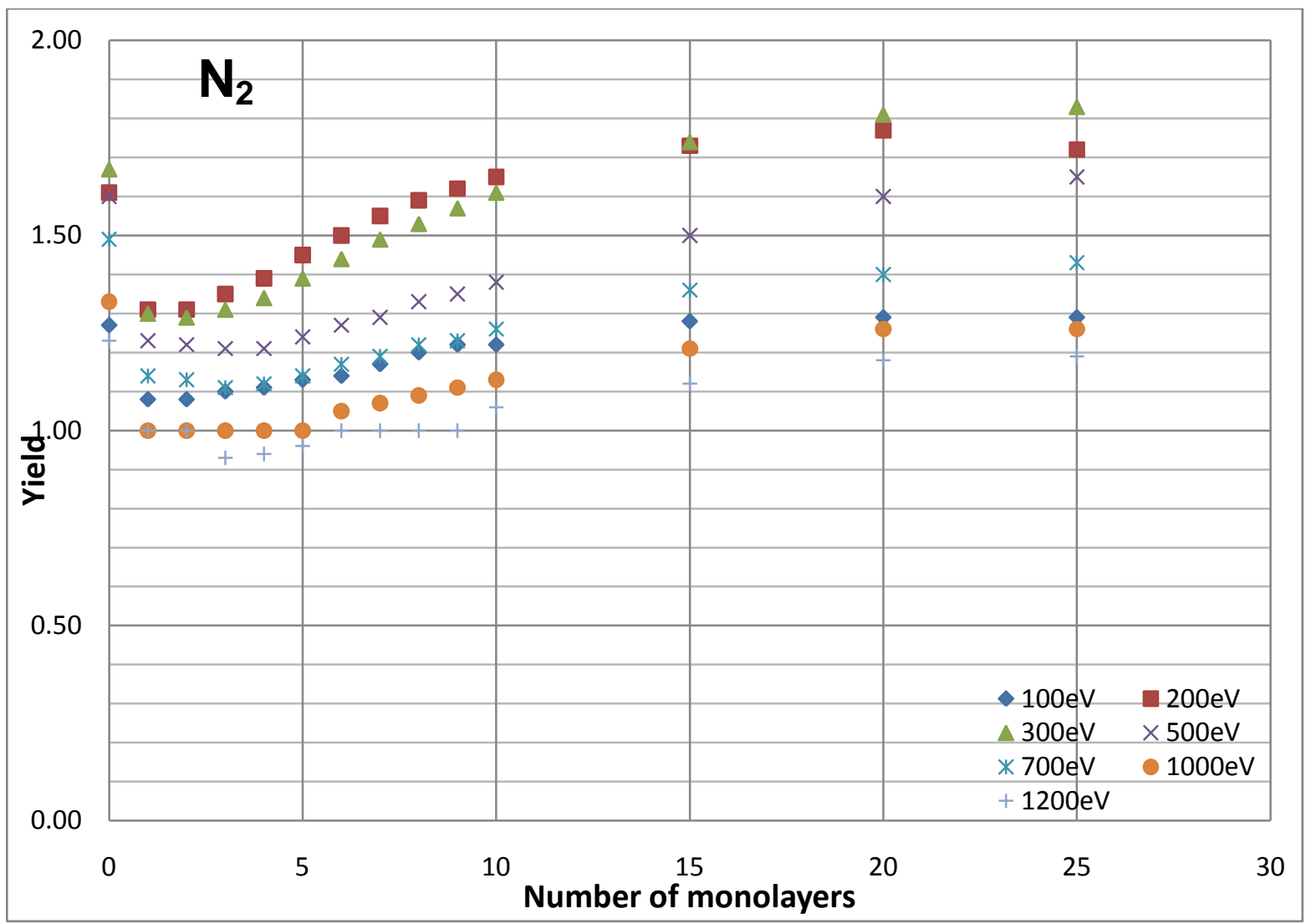

Figure 5.71: SEY of $\mathrm{N}_{2}$ as a function of coverage for defined primary electron energies.

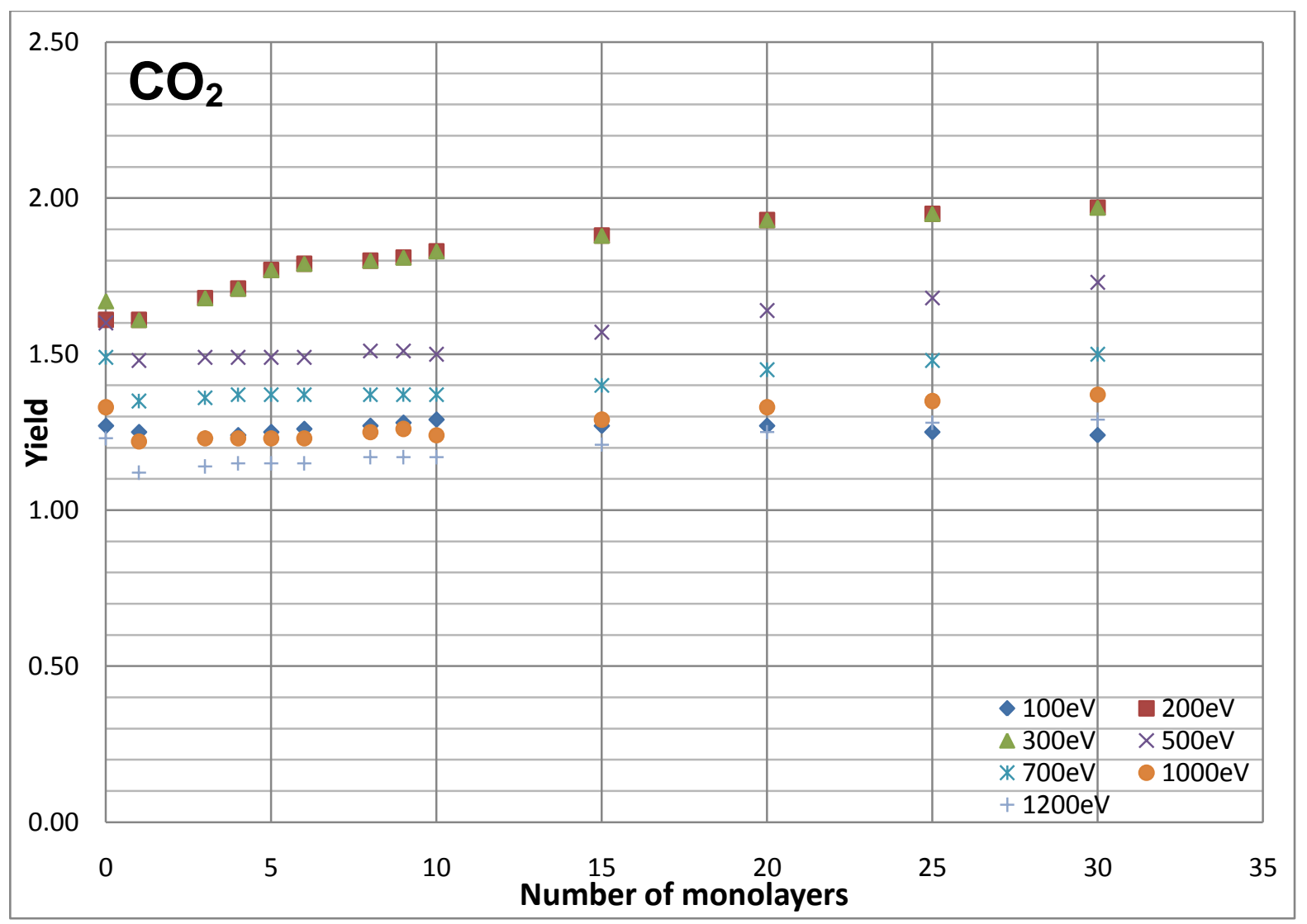

Figure 5.72: $\mathrm{SEY}$ of $\mathrm{CO}_{2}$ as a function of coverage for defined primary electron energies. 
Chapter 5-Results

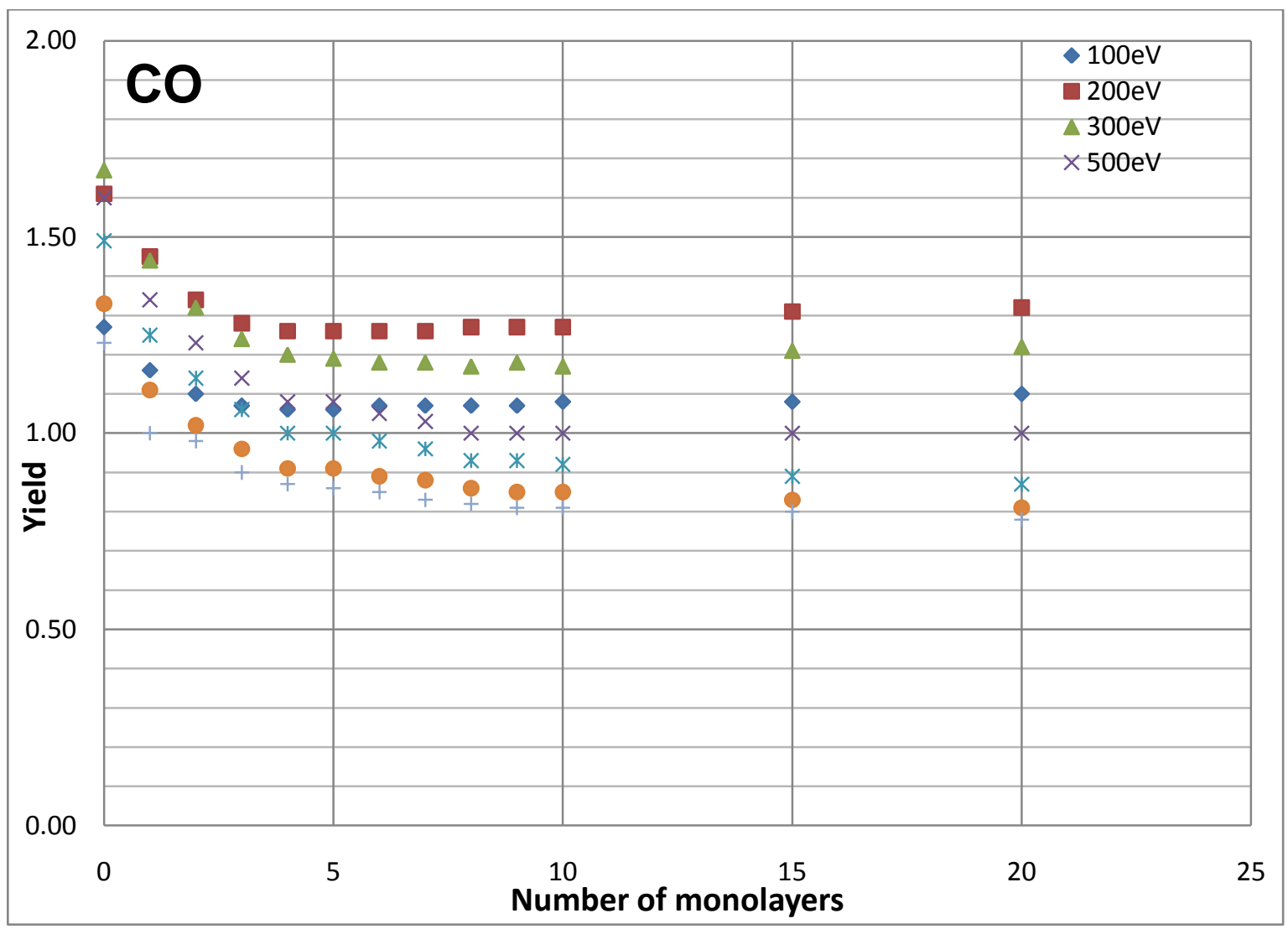

Figure 5.73: SEY of CO as a function of coverage for defined primary electron energies.

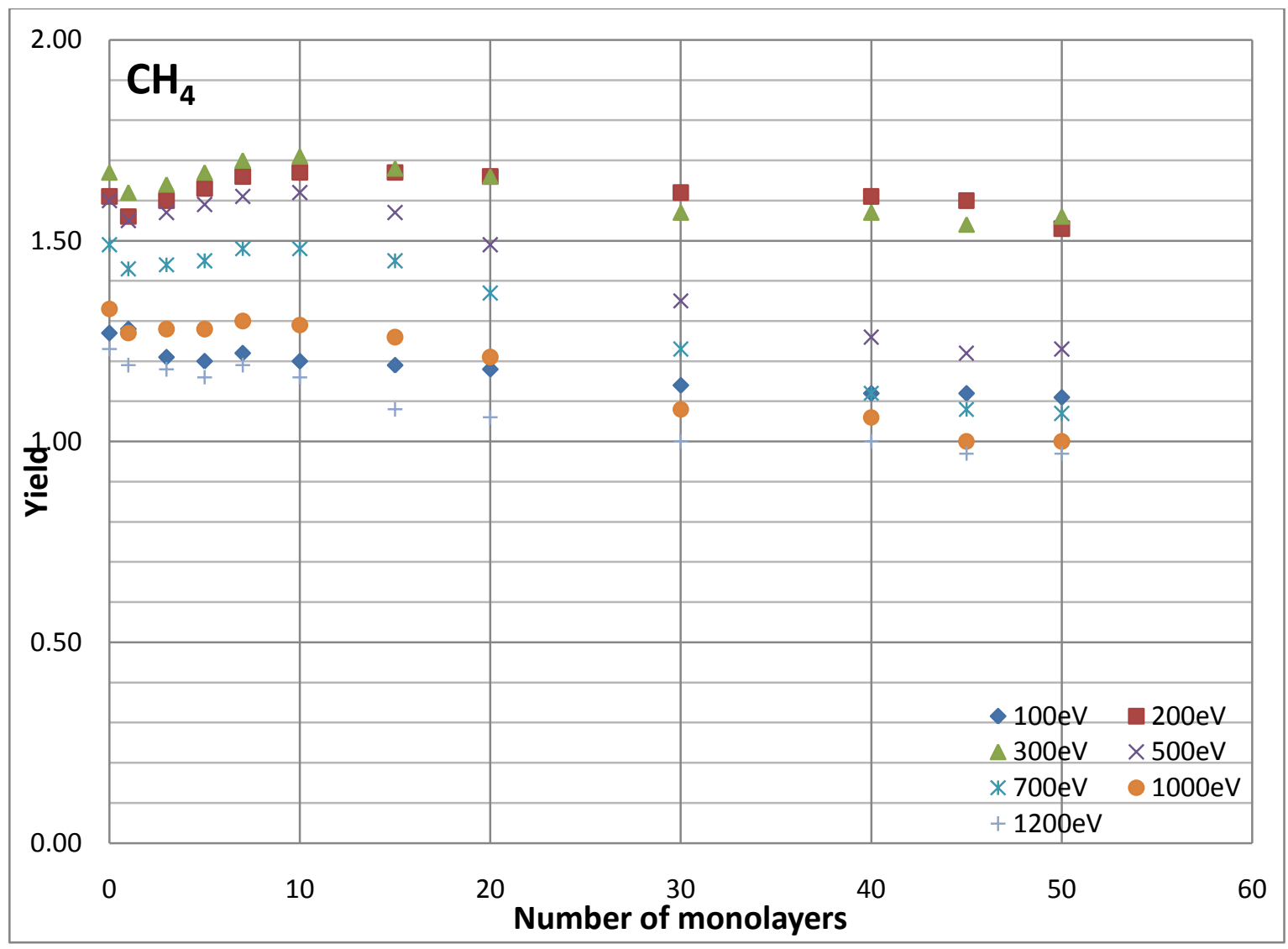

Figure 5.74: $\mathrm{SEY}$ of $\mathrm{CH}_{4}$ as a function of coverage for defined primary electron energies. 
Chapter 5-Results

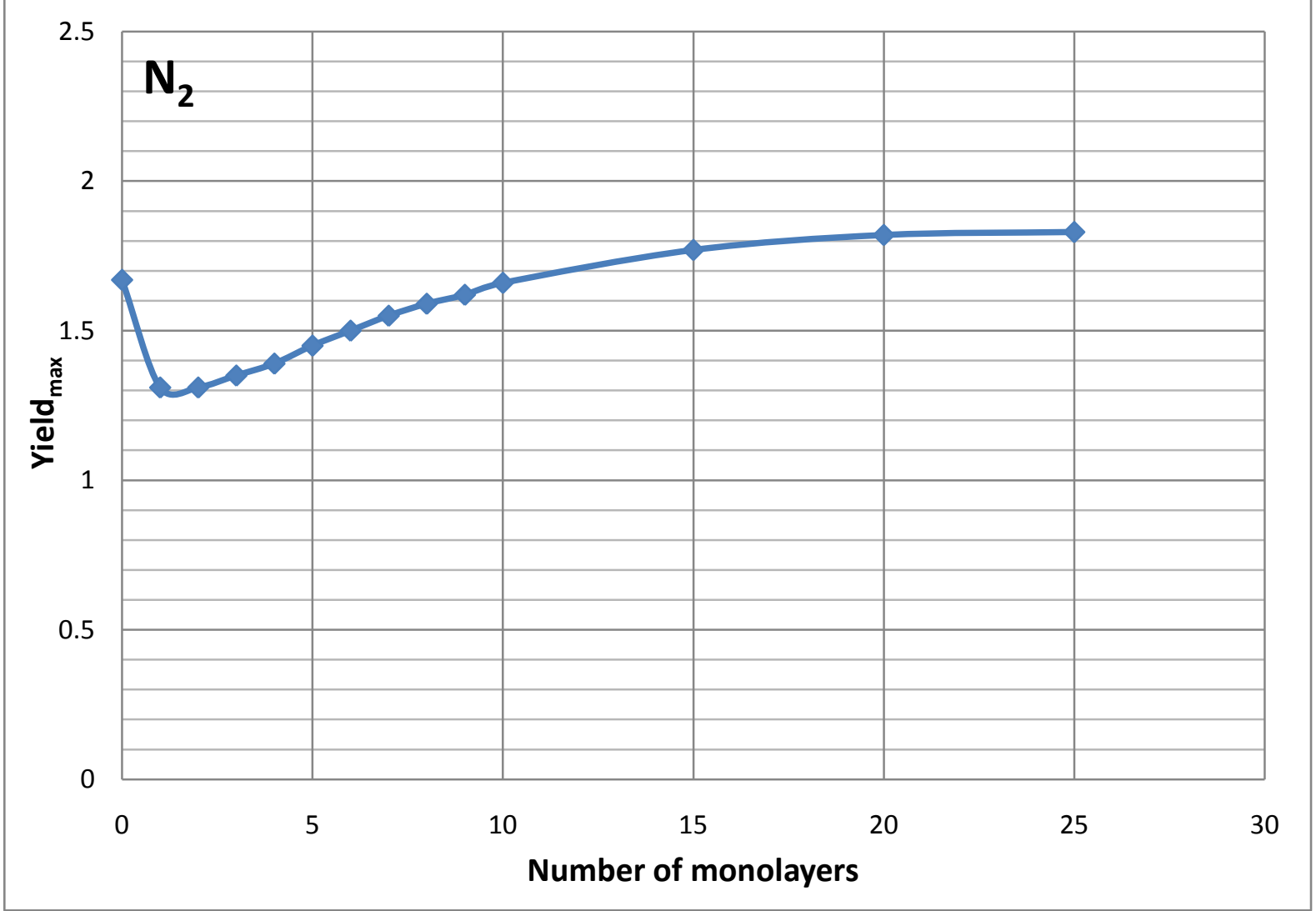

Figure 5.75: Maximum yield of $\mathrm{N}_{2}$ for each number of monolayer.

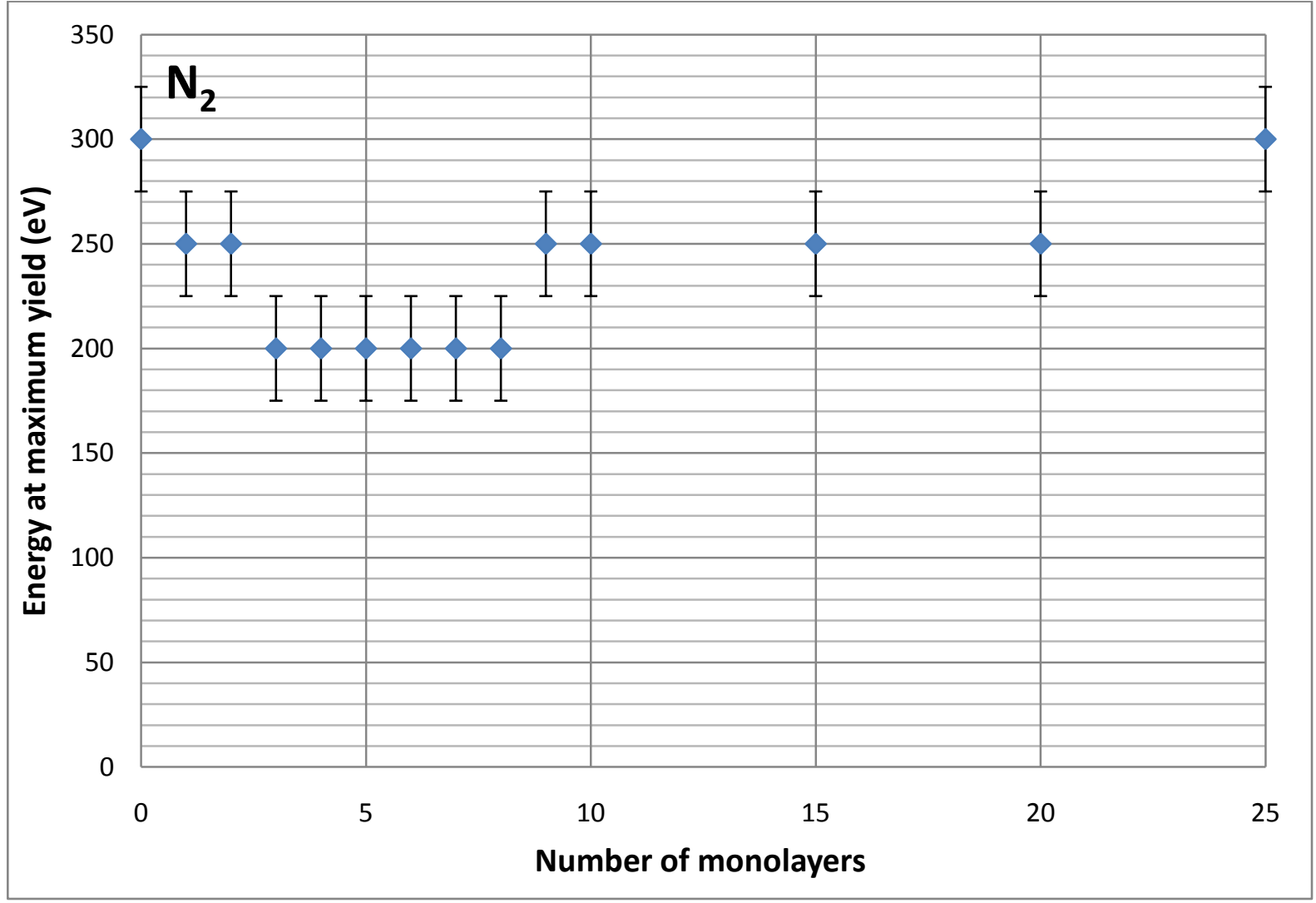

Figure 5.76: Energy at the maximum yield of $\mathrm{N}_{2}$ as a function of coverage. 
Chapter 5-Results

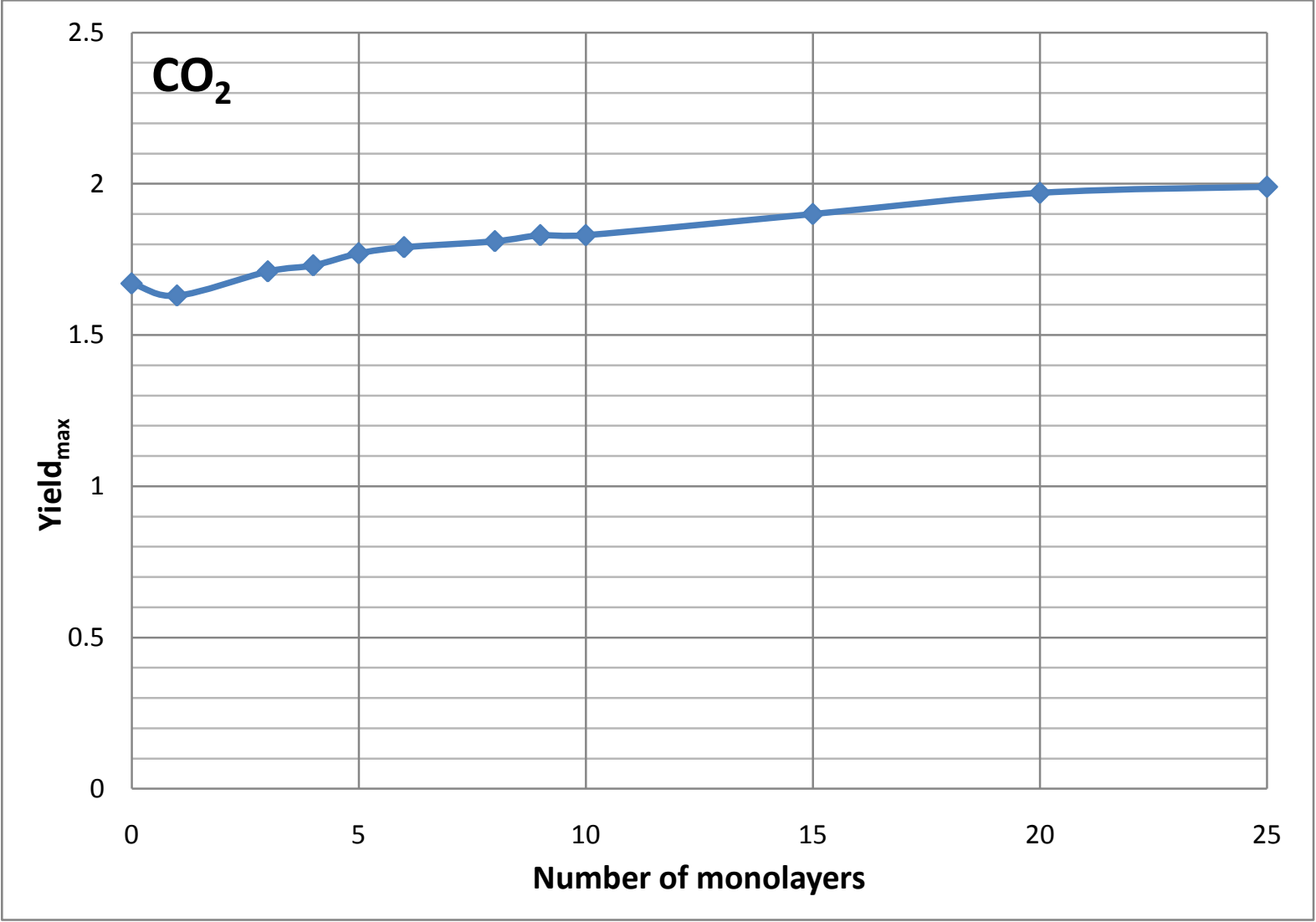

Figure 5.77: Maximum yield of $\mathrm{CO}_{2}$ for each number of monolayer.

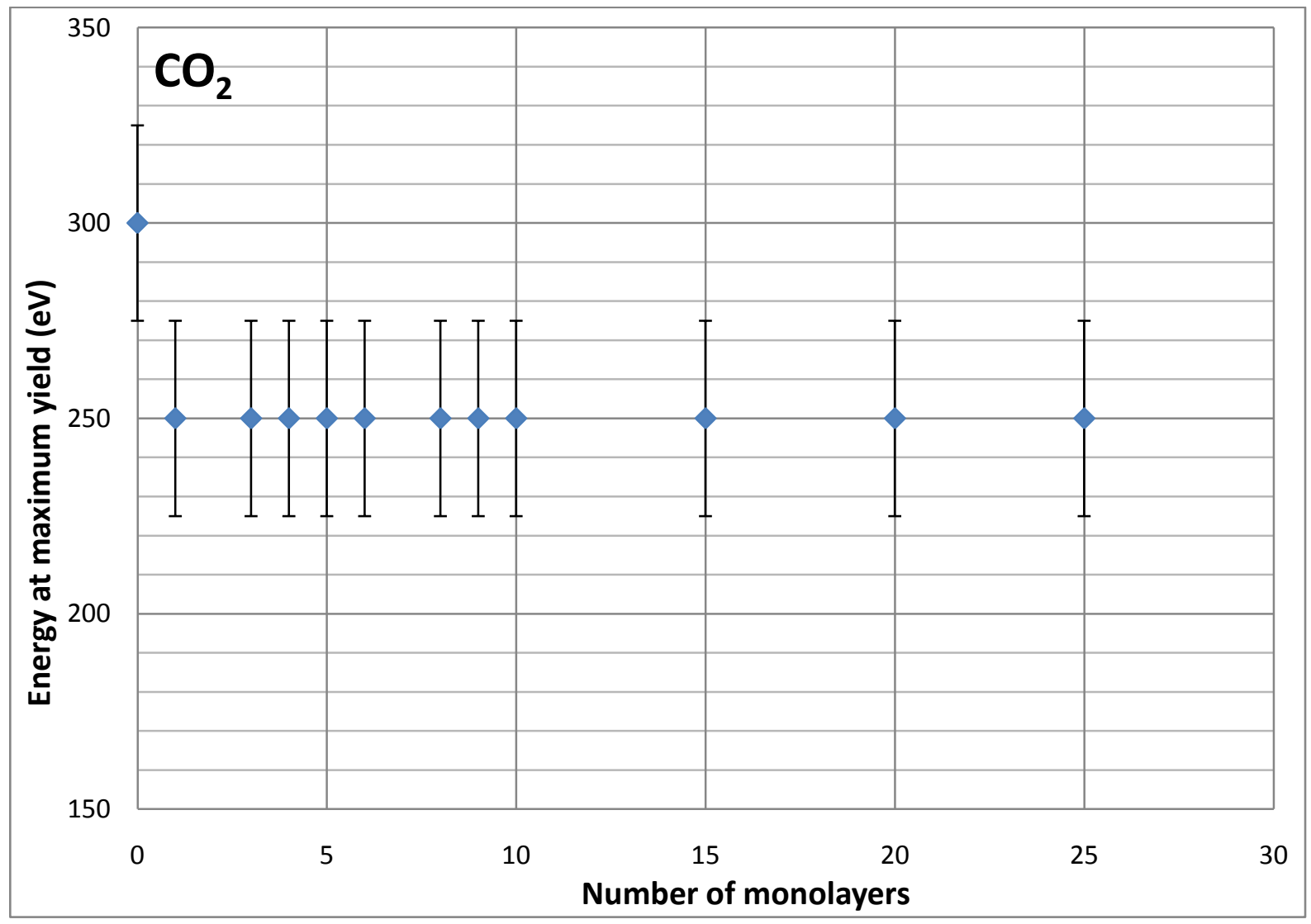

Figure 5.78: Energy at the maximum yield of $\mathrm{CO}_{2}$ as a function of coverage. 
Chapter 5-Results

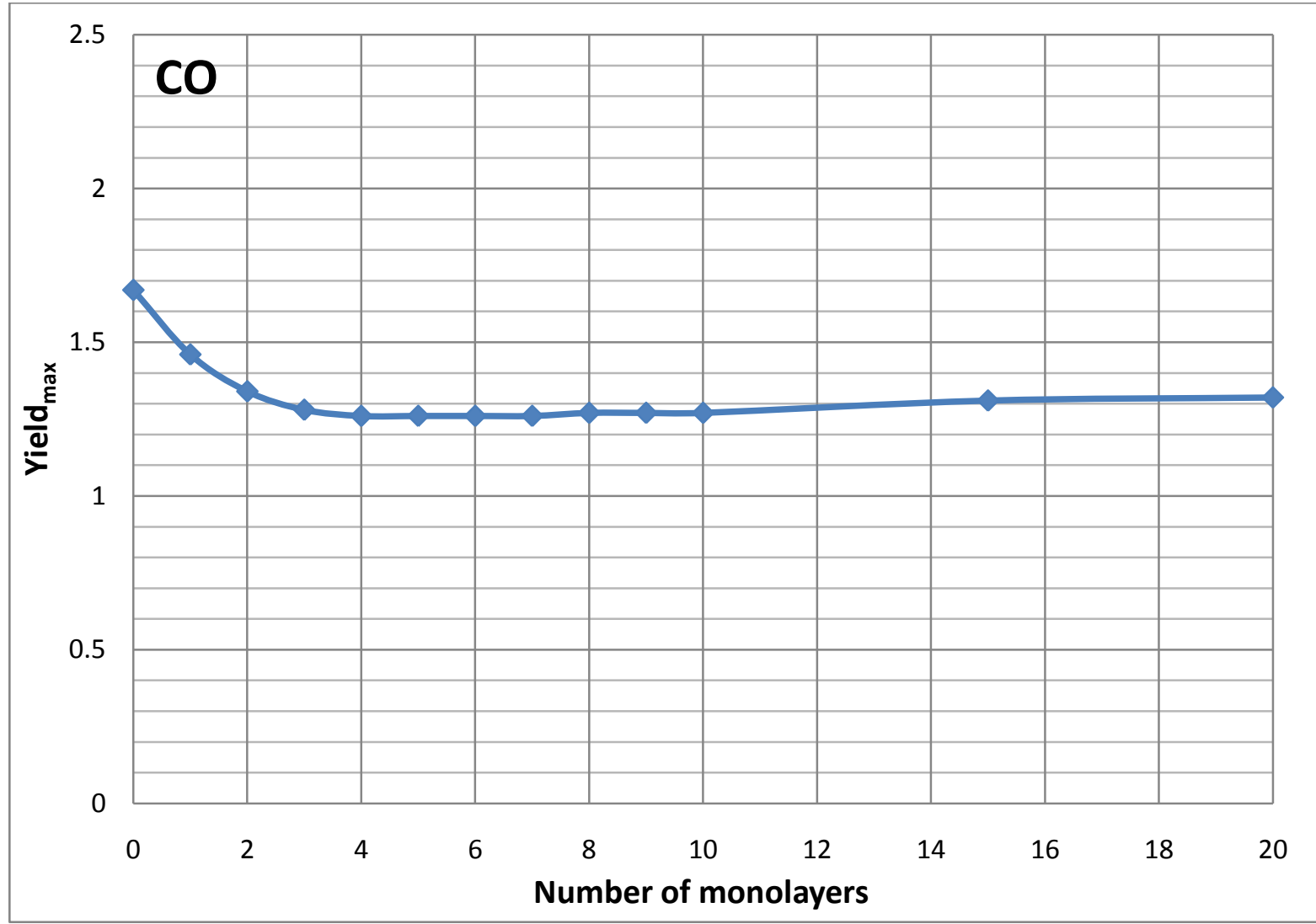

Figure 5.79: Maximum yield of $\mathrm{CO}$ for each number of monolayer.

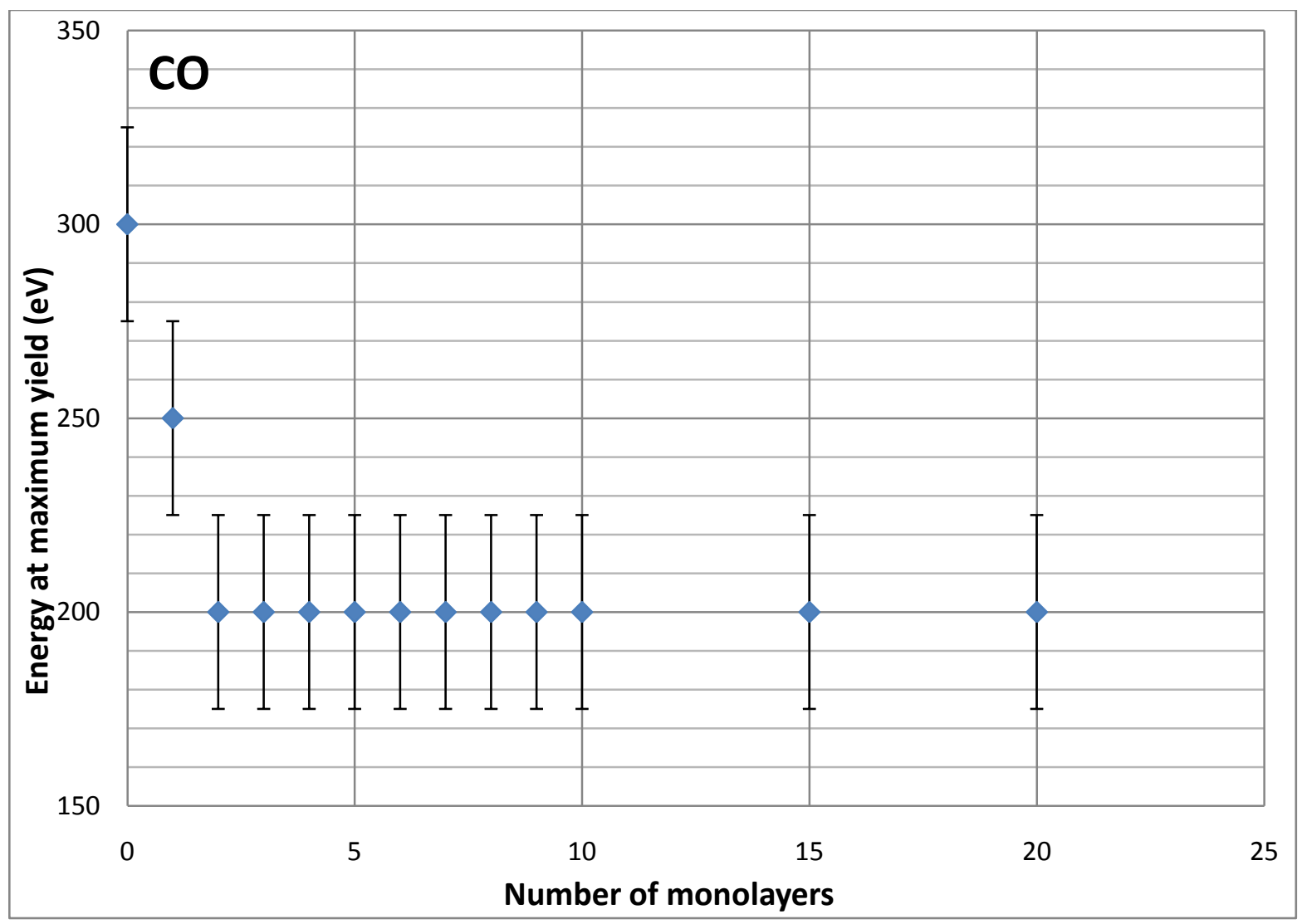

Figure 5.80: Energy at the maximum yield of $\mathrm{CO}$ as a function of coverage. 


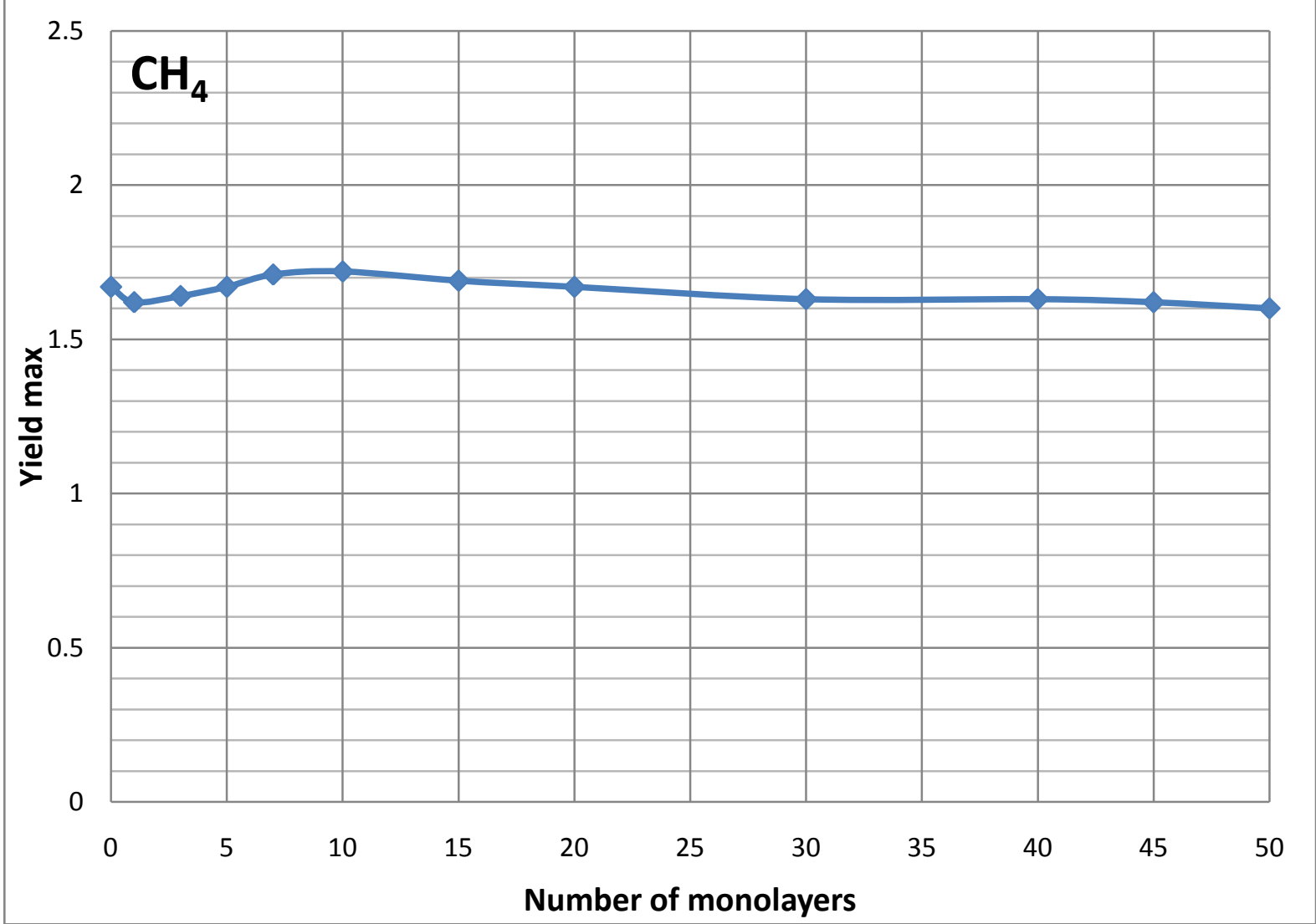

Figure 5.81: Maximum yield of $\mathrm{CH}_{4}$ for each number of monolayer.

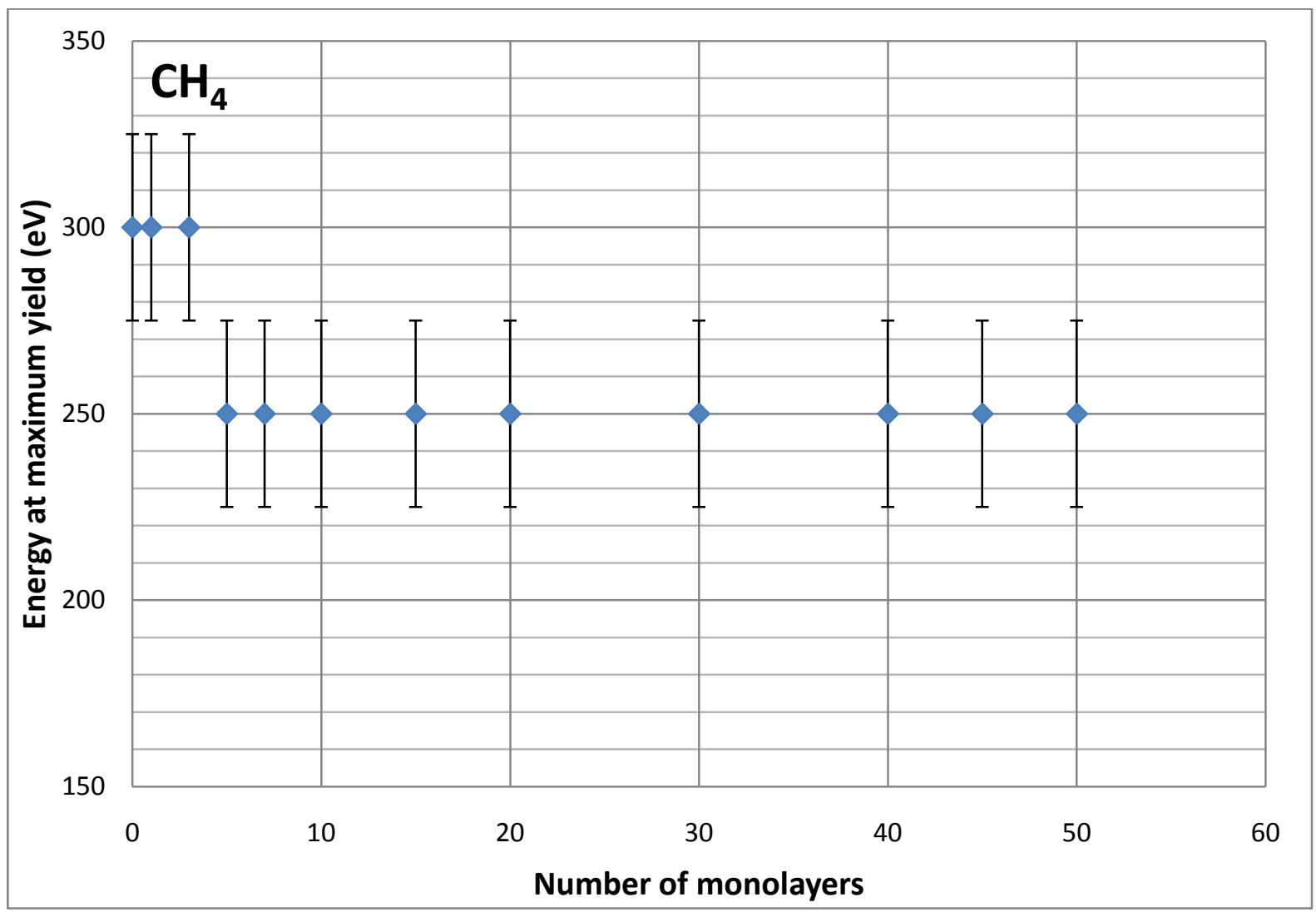

Figure 5.82: Energy at the maximum yield of $\mathrm{CH}_{4}$ as a function of coverage. 


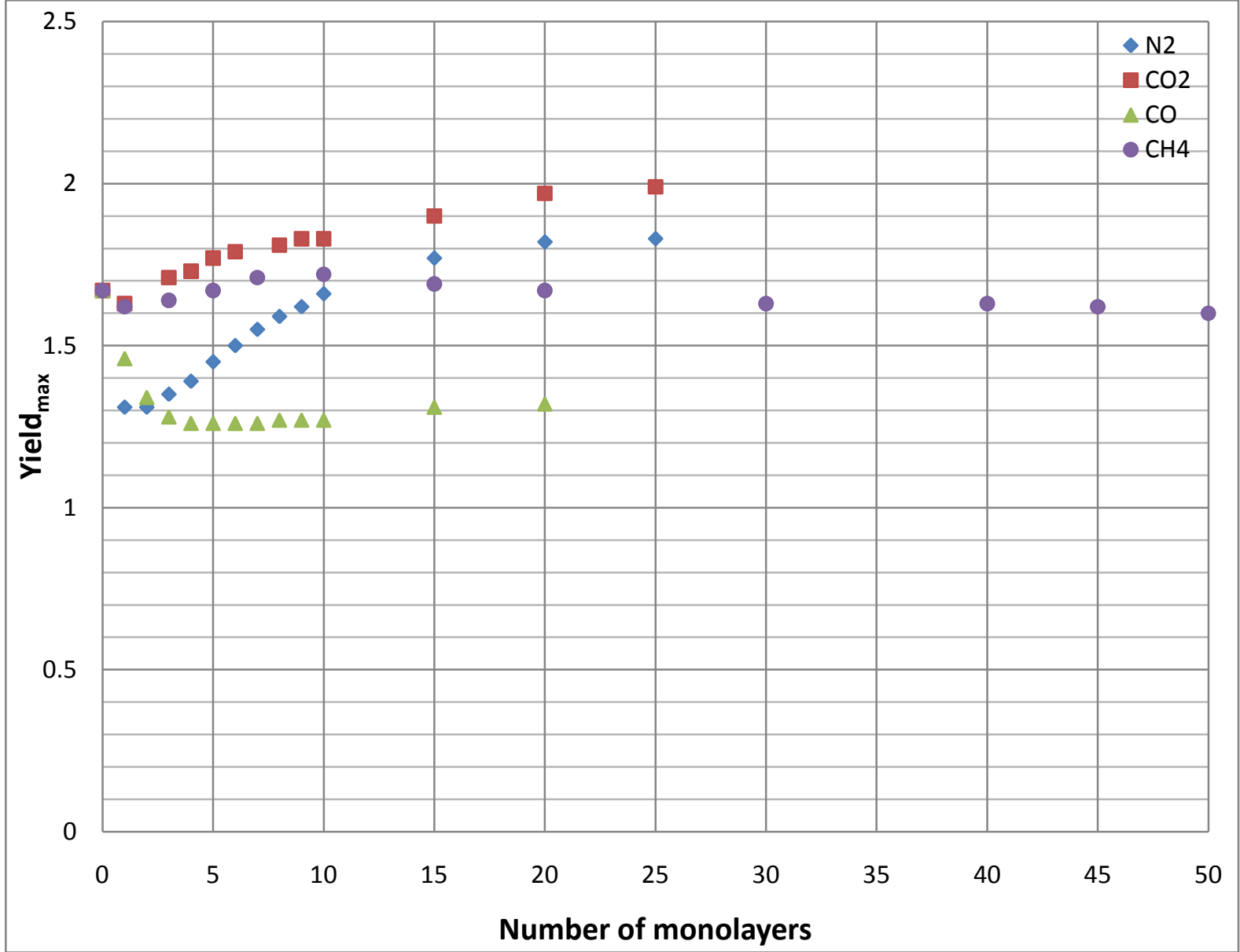

Figure 5.83: Maximum yield as a function of coverage for different adsorbates.

\subsubsection{Comparison of the SEY of different substrates}

The secondary electron yield of copper, aluminium and electro polished copper as a function of physisorbed gases are compared in the following figures (Figure 5.84, 5.85, 5.86, 5.87). The SEY of the first monolayers are changing depending on the substrate material. With increasing number of monolayers the yield for different materials gets more and more the same. The reason is that the yield with few monolayers is the total yield of the substrate and the physisorbed gas. Whereas with more monolayers, the substrate loses the importance and the physisorbed gas becomes the dominant factor in the yield. Finally after reaching the number of monolayers for saturation the yield for each substrate becomes the same. 
Chapter 5-Results

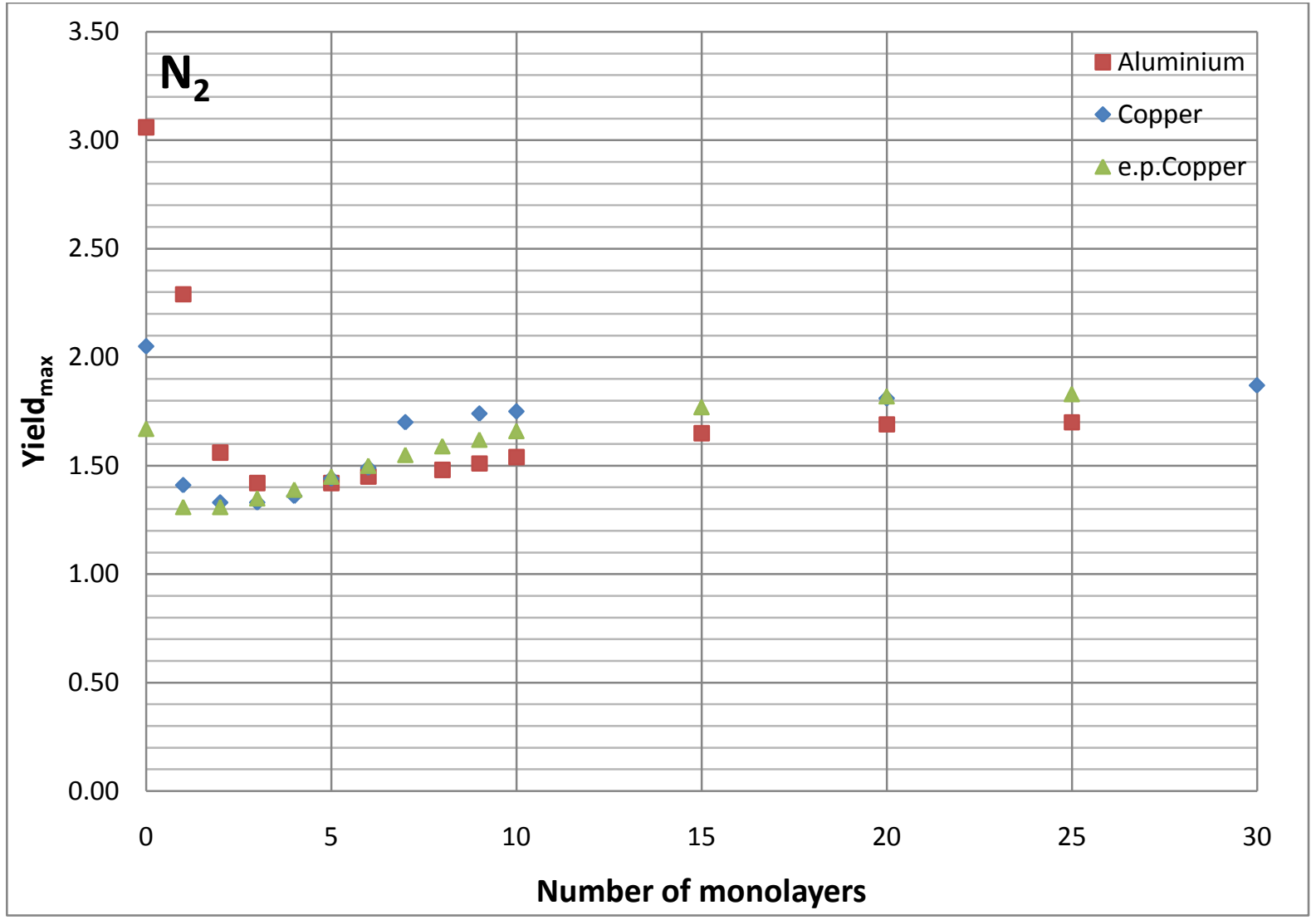

Figure 5.84: Maximum yield of different $N_{2}$ coverages physisorbed on different substrates.

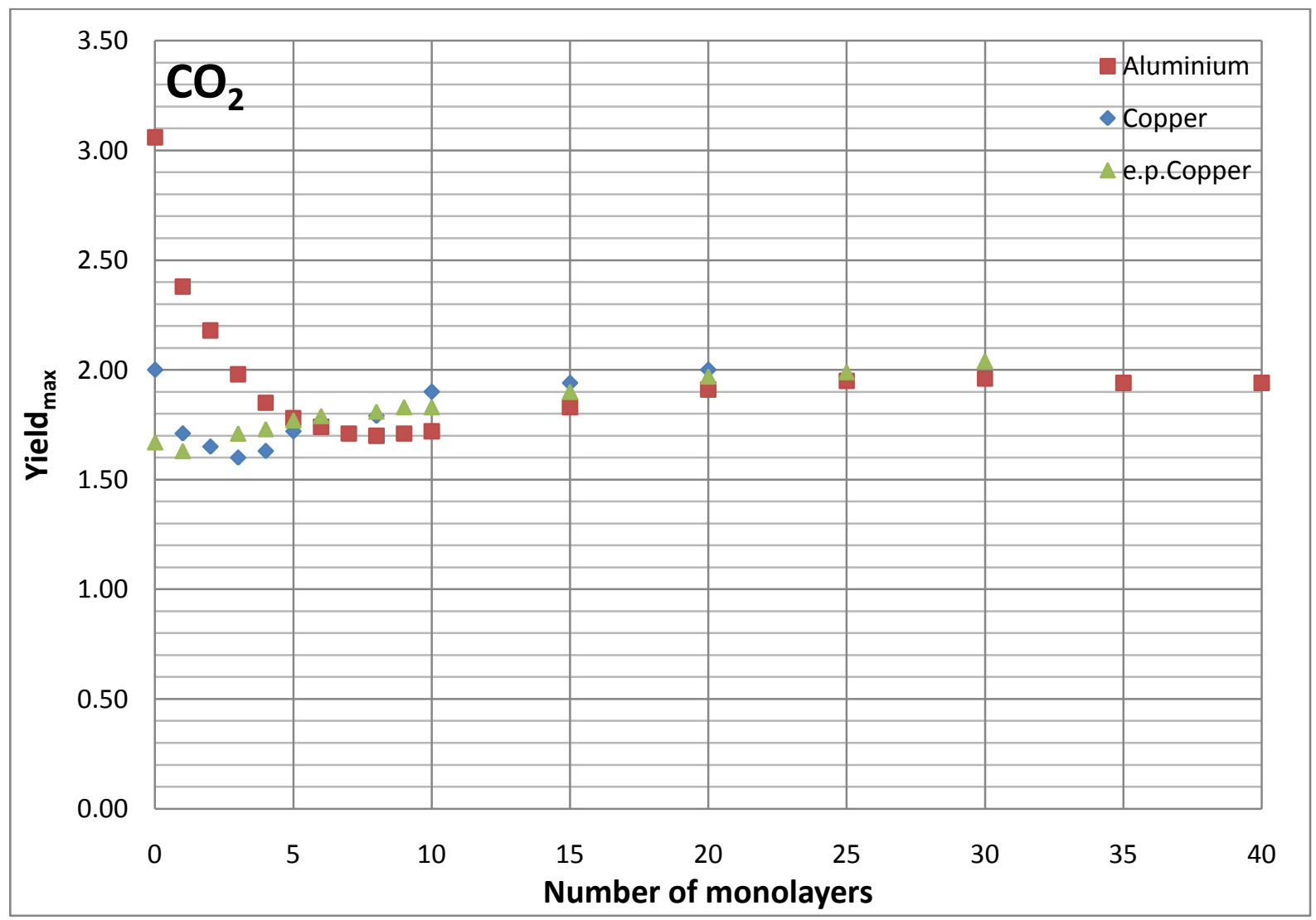

Figure 5.85: Maximum yield of different $\mathrm{CO}_{2}$ coverages physisorbed on different substrates. 


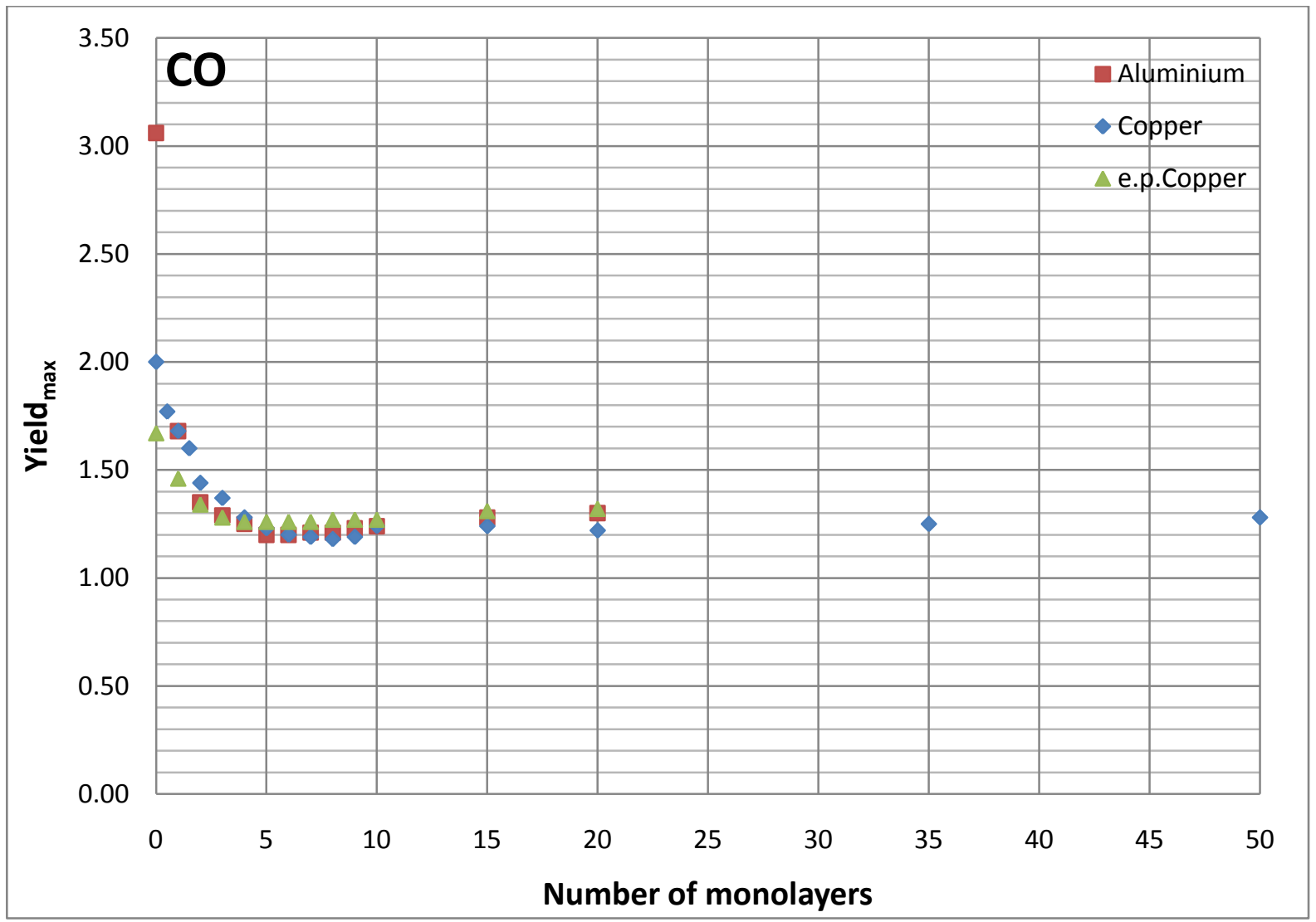

Figure 5.86: Maximum yield of different CO coverages physisorbed on different substrates.

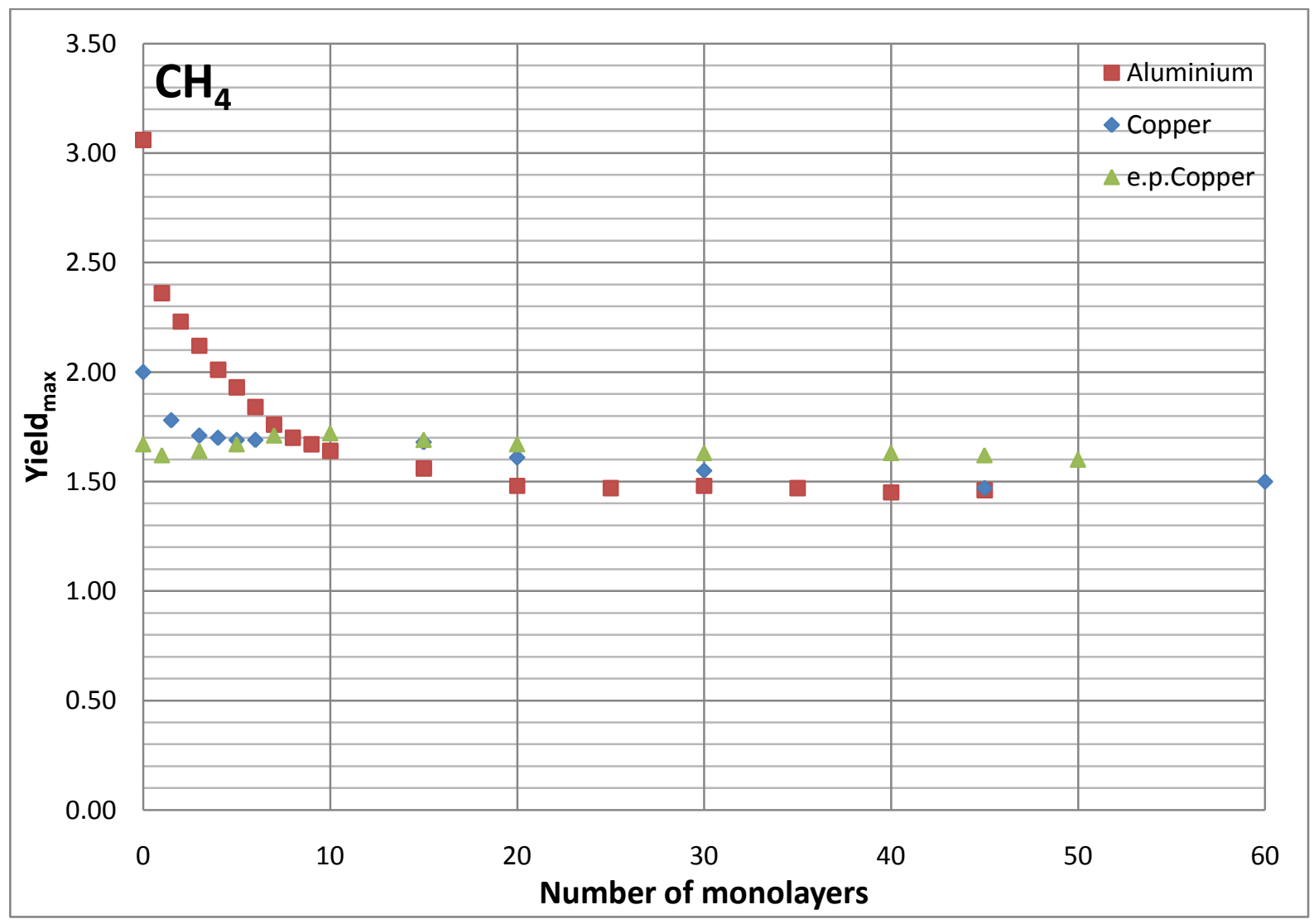

Figure 5.87: Maximum yield of different $\mathrm{CH}_{4}$ coverages physisorbed on different substrates. 


\subsubsection{Work function}

Work function changes could be in principle one of the possible explanations for the change of SEY at low coverage for different adsorbates. Due to the lacking of experimental data, measurements with a Kelvin Probe have been performed to evaluate the importance of this effect. A major difficulty which was encountered is the charging of the adsorbates.

For the following measurements, the sample has been cooled down to $4,7 \mathrm{~K}$. After reaching that temperature the gas, of which we wanted to measure the work function, has been adsorbed on the cold sample. Once reaching the desired amount of layers, the gas inlet has been closed. After placing the tip of the Kelvin Probe in front of the sample, the measurement has been started. While continuously measuring the work function, the sample has been slowly warmed up. The reason for warming up the sample is to avoid the charging of the adsorbate, which can occur due to the work function measurement.

The measured values for the work function are relative values, because the Kelvin Probe was not calibrated. The work function of the cold electro polished copper at cryogenic temperatures is $6070 \mathrm{meV}$ relatively. At approx. $1 \cdot 10^{-8} \mathrm{mbar}$ pressure, which is the partial pressure of each gas during measurements, evaporation exceeds condensation for $\mathrm{N}_{2}$ above $21 \mathrm{~K}$, for $\mathrm{CO}$ above $25 \mathrm{~K}$ and for $\mathrm{CO}_{2}$ above $72 \mathrm{~K}$.

The given values of the work function are the relative change of the work function of the substrate as a function of different adsorbates. They are read from the following curves of each gas just before reaching the temperature for their evaporation. $\Delta \Phi$ of $\mathrm{N}_{2}$ is $64 \mathrm{meV}, \Delta \Phi$ of $\mathrm{CO}$ is $78 \mathrm{meV}$ and $\Delta \Phi$ of $\mathrm{CO}_{2}$ is $50 \mathrm{meV}$. The standard deviation is about $15 \mathrm{meV}$.

Figures 5.88, 5.89, 5.90 and 5.91 show the charts of the work function while warming with and without charging of electro-polished copper, $\mathrm{N}_{2}, \mathrm{CO}$ and $\mathrm{CO}_{2}$. 
Chapter 5-Results

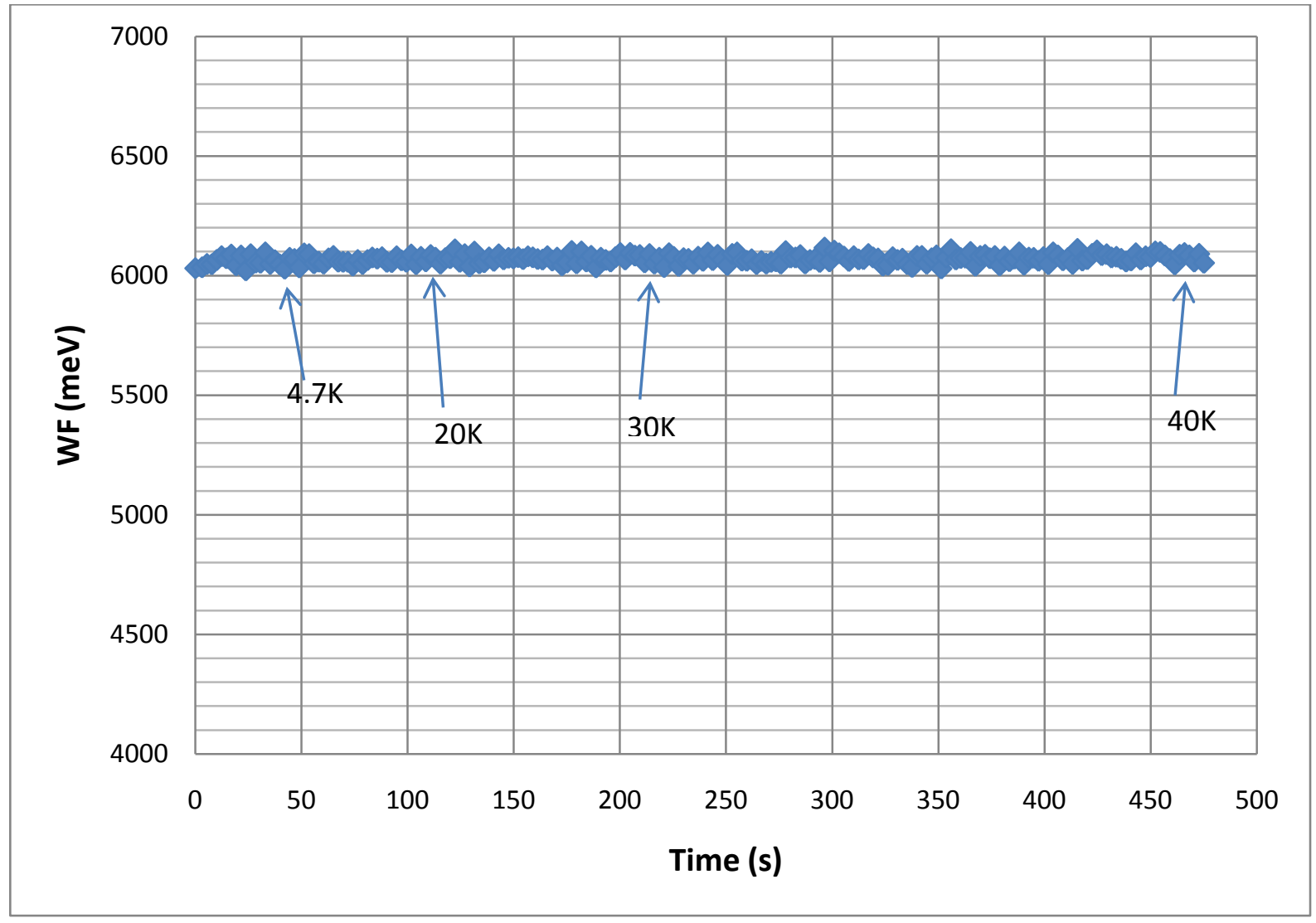

Figure 5.88: Work function of electro polished copper.

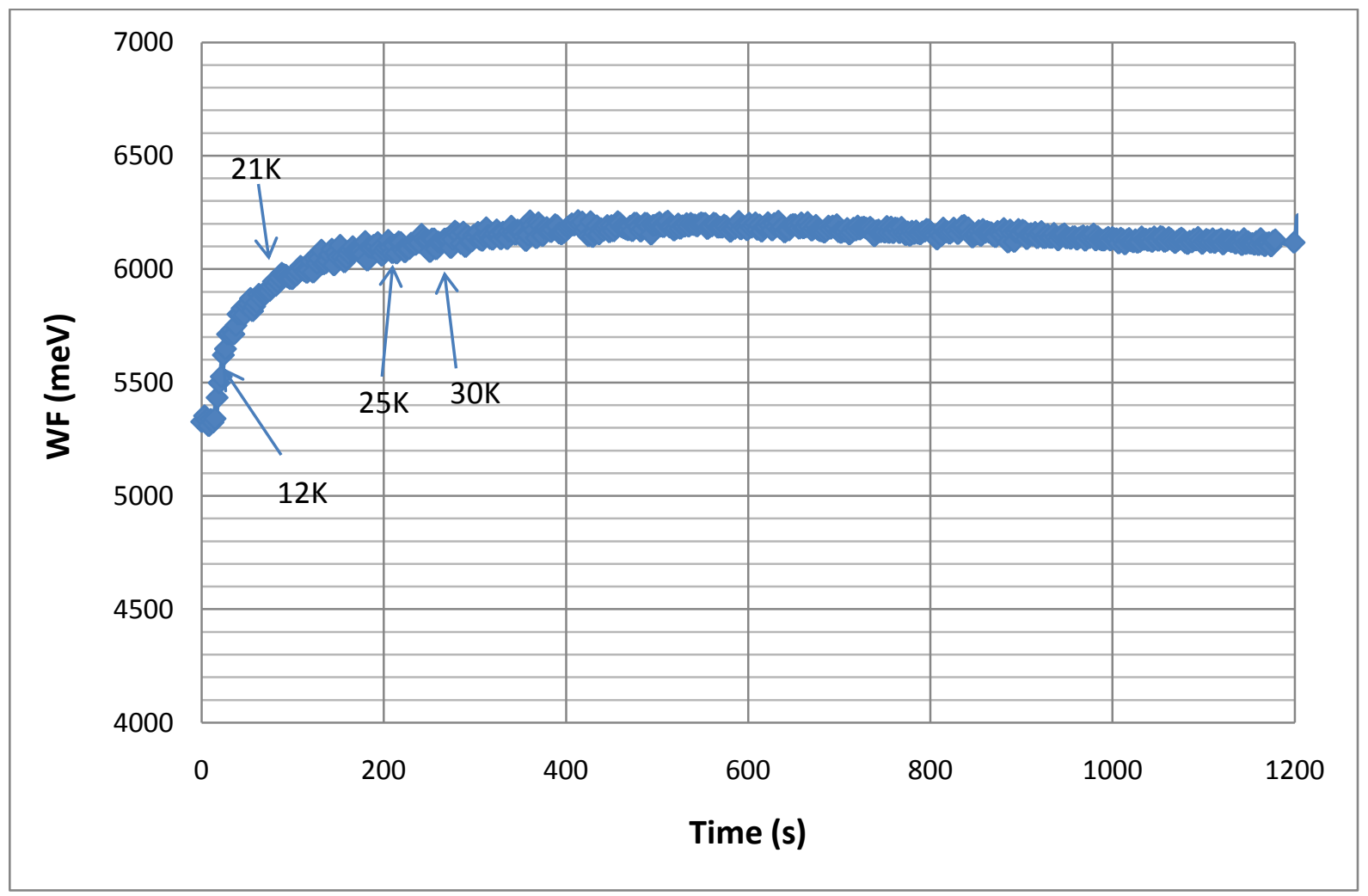

Figure 5.89: Work function of CO. 


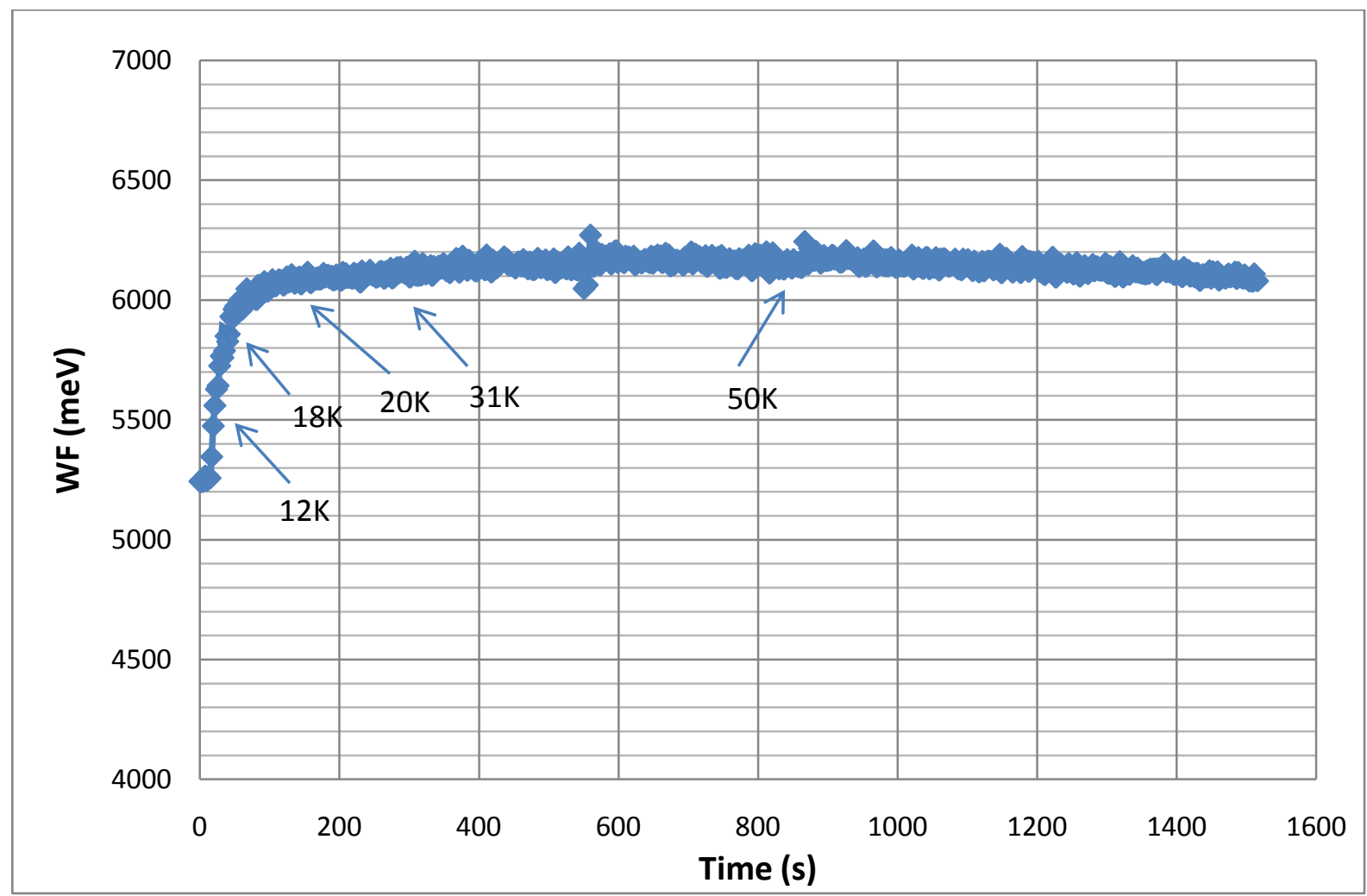

Figure 5.90: Work function of $\mathrm{N}_{2}$.

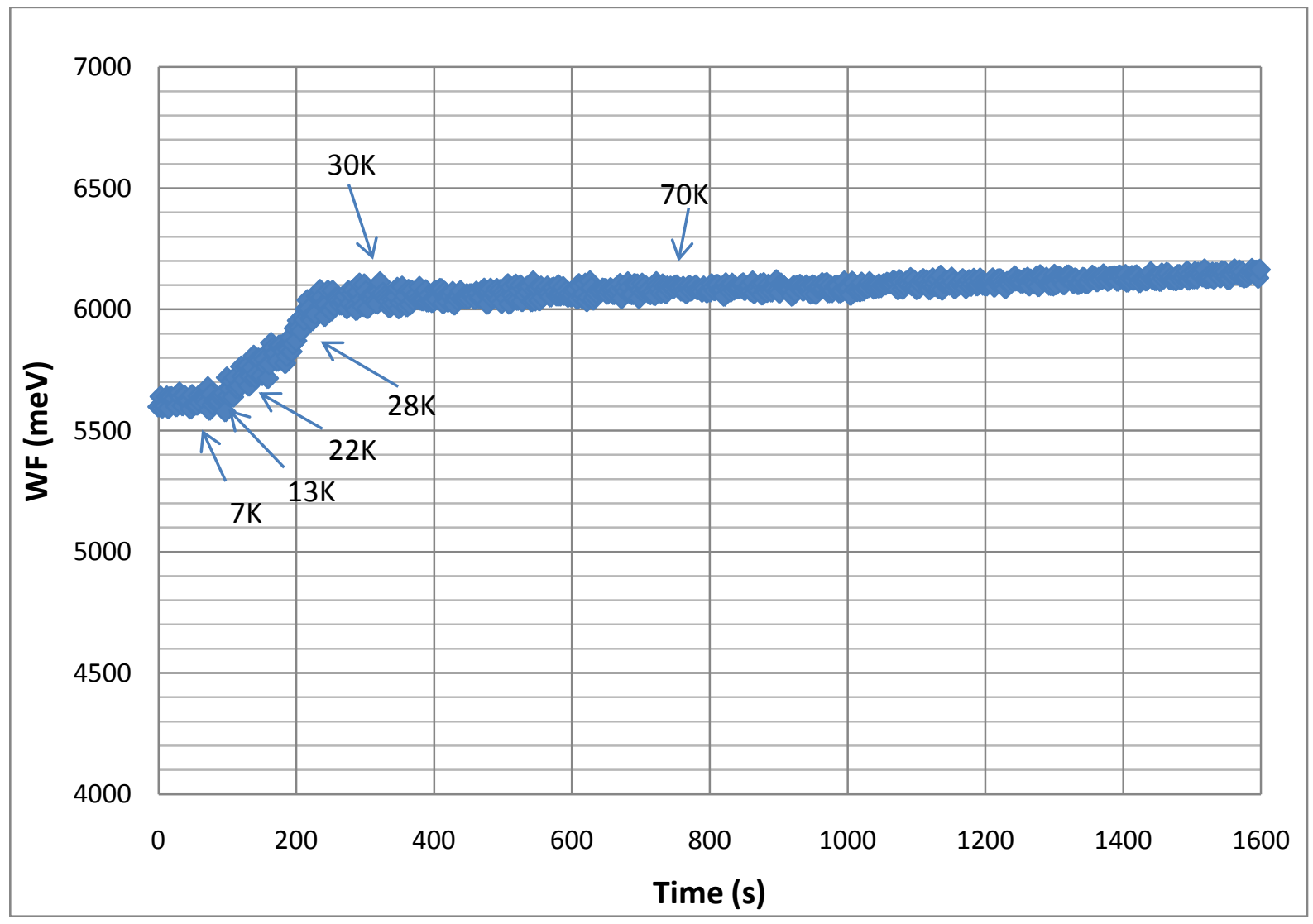

Figure 5.91: Work function of $\mathrm{CO}_{2}$. 


\subsubsection{SEY of Niobium}

Niobium is a metal in group 5 of the periodic table, although it has an atypical configuration in its outermost electron shells compared to the rest of the members. It becomes a superconductor Type II at cryogenic temperatures. The critical temperature at atmospheric pressure is $9.2 \mathrm{~K}$.

The niobium sample has been cooled down to $6 \mathrm{~K}$ and its SEY has been measured at different temperatures. It is important to note that the Niobium sample was with an oxide layer. The question was, if it shows a different behaviour at the critical temperature or if the results show that its behaviour is the same as all other metals. The yield decreases a little bit with decreasing temperature but the reason for that is again the adsorption of the rest gases in the vacuum chamber. The results for different temperatures are shown below in Figure 5.92 .

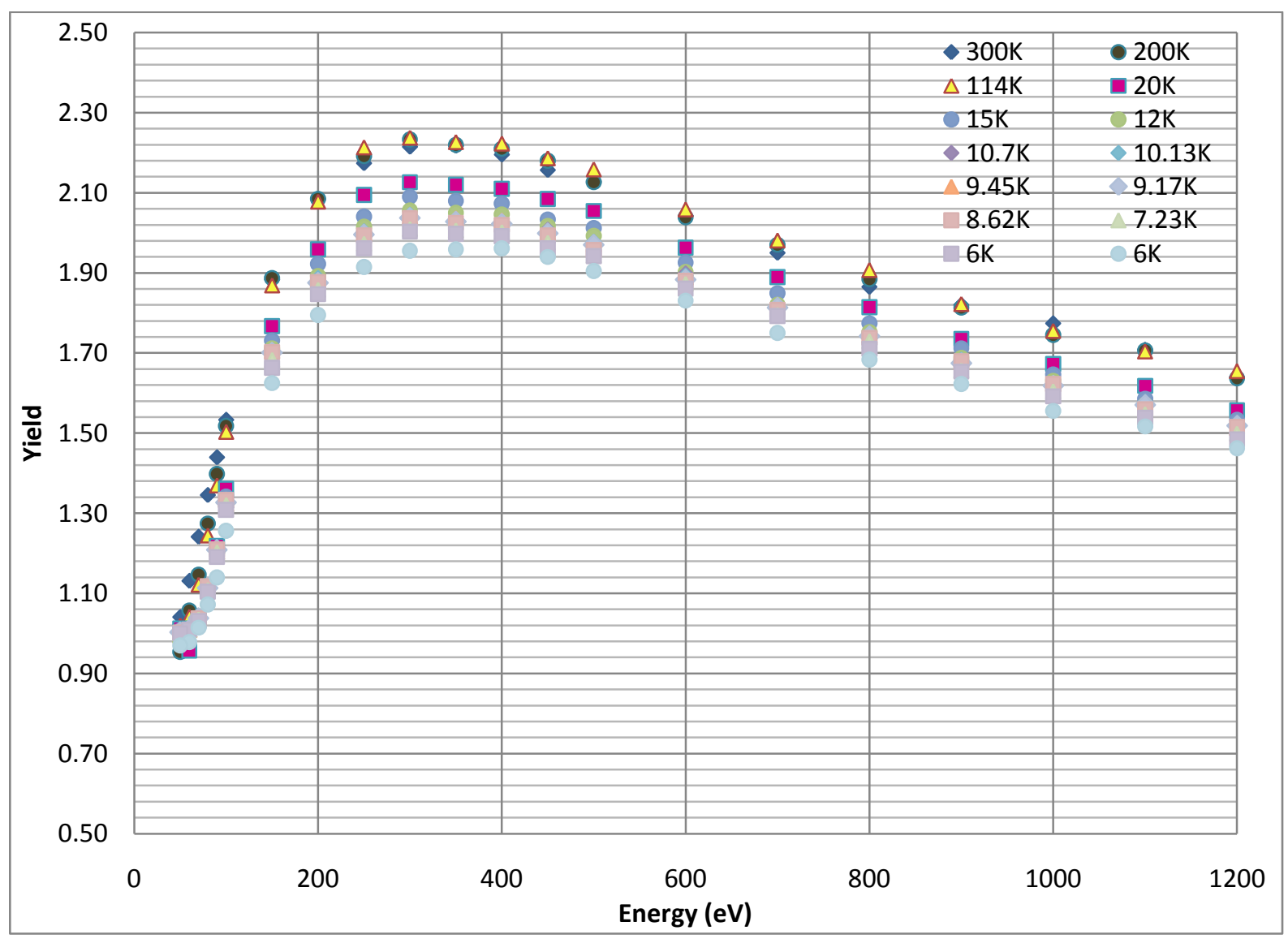

Figure 5.92: SEY of Niobium as a function of the primary electron energy at different temperatures. 


\subsubsection{SEY of carbon}

In order to eliminate the electron cloud effect, a material with a SEY less than 1.3 is necessary (G.Rumolo). Carbon is a material with very low SEY. It is a robust material and has a long life time. Therefore it can be considered for use in future updates of LHC. The behaviour of carbon at room temperatures has been measured already but there are still no data about the behaviour at cryogenic temperatures. To cover this lack of data, measurements with carbon at $4.7 \mathrm{~K}$ have been performed.

A copper sample has been coated by the Mechanical and Materials Engineering group with graphite-like amorphous carbon films. The thickness of the coating is $400 \mathrm{~nm}$. After the coating, the sample has been placed in the vacuum chamber and the system has been baked out.

In figure 5.93, the SEY of carbon at room temperature and at cryogenic temperature has been compared.

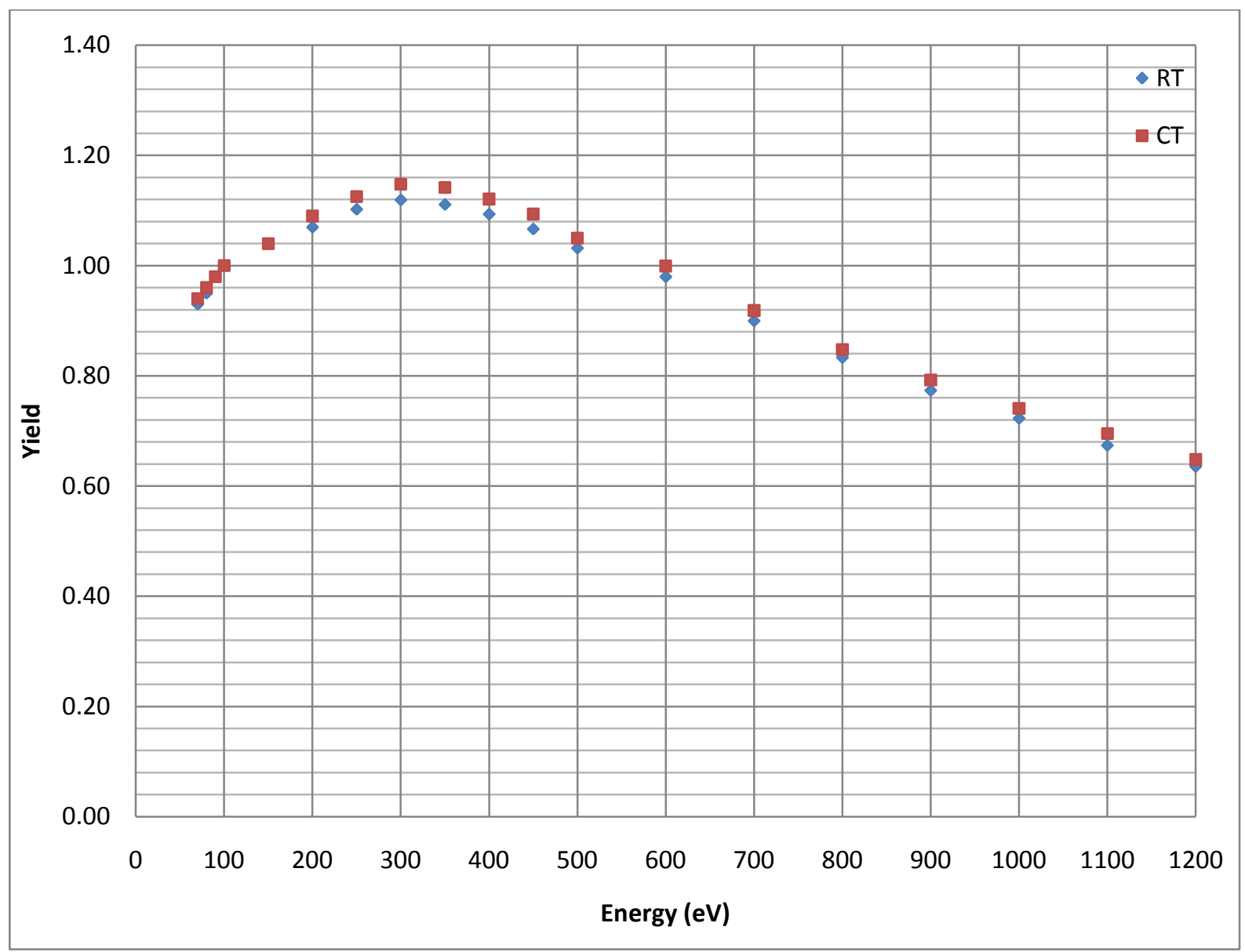

Figure 5.93: SEY of carbon at room temperature and at cryogenic temperatures. 
The SEY of carbon at cryogenic temperatures is slightly higher than at room temperatures. The reason could be again the adsorption of residual gases in the vacuum chamber.

The measured SEY of carbon is higher than the existing results. The SEY of the same sample has been measured using another system and the results show the same value. Therefore, the reason could be the surface morphology of the coating.

\subsubsection{Adsorption and conditioning of physisorbed layers}

For this measurement the following gases were used: $\mathrm{H}_{2} \mathrm{O}, \mathrm{CO}$ and $\mathrm{N}_{2}$. To do the conditioning, 25 monolayers of water have been physisorbed on the cold electro polished copper substrate.

The water injection system, used for the adsorption, has been described in chapter 4. After adsorbing water, the substrate has been bombarded with an electron beam of energy $300 \mathrm{eV}$.

The current of the beam was around $1.6 \cdot 10^{-5} \mathrm{~A}$ on an area of $1 \mathrm{~mm}^{2}$. This high beam current could be reached by using an electron gun filament current of $2,4 \mathrm{~A}$. The beam used for conditioning was not pulsed. The sample has been bombarded several times in steps of 100s with SEY measurements in between. While doing the SEY measurement the beam was pulsed and the electron gun current was $2.2 \mathrm{~A}$.

The residual gas pressure in the vacuum chamber before the conditioning and before the water injection was about $3.4 \cdot 10^{-9} \mathrm{mbar}$. During water injection it increased to $2 \cdot 10^{-7} \mathrm{mbar}$. After adsorption and stopping the gas inlet it decreases again down to $4 \cdot 10^{-9} \mathrm{mbar}$. During conditioning under electron bombardment the partial water pressure was around $3 \cdot 10^{-8} \mathrm{mbar}$ due to desorption of the molecules. Therefore interruption of irradiation caused an immediate increase of SEY due to water re-adsorption.

Figure 5.94 shows the spectra before and during water injection. Figure 5.95 shows the maximum yield as a function of the electron dose. Finally Figure 5.96 shows the secondary electron yield of 25 monolayers of water during conditioning. 
Chapter 5-Results

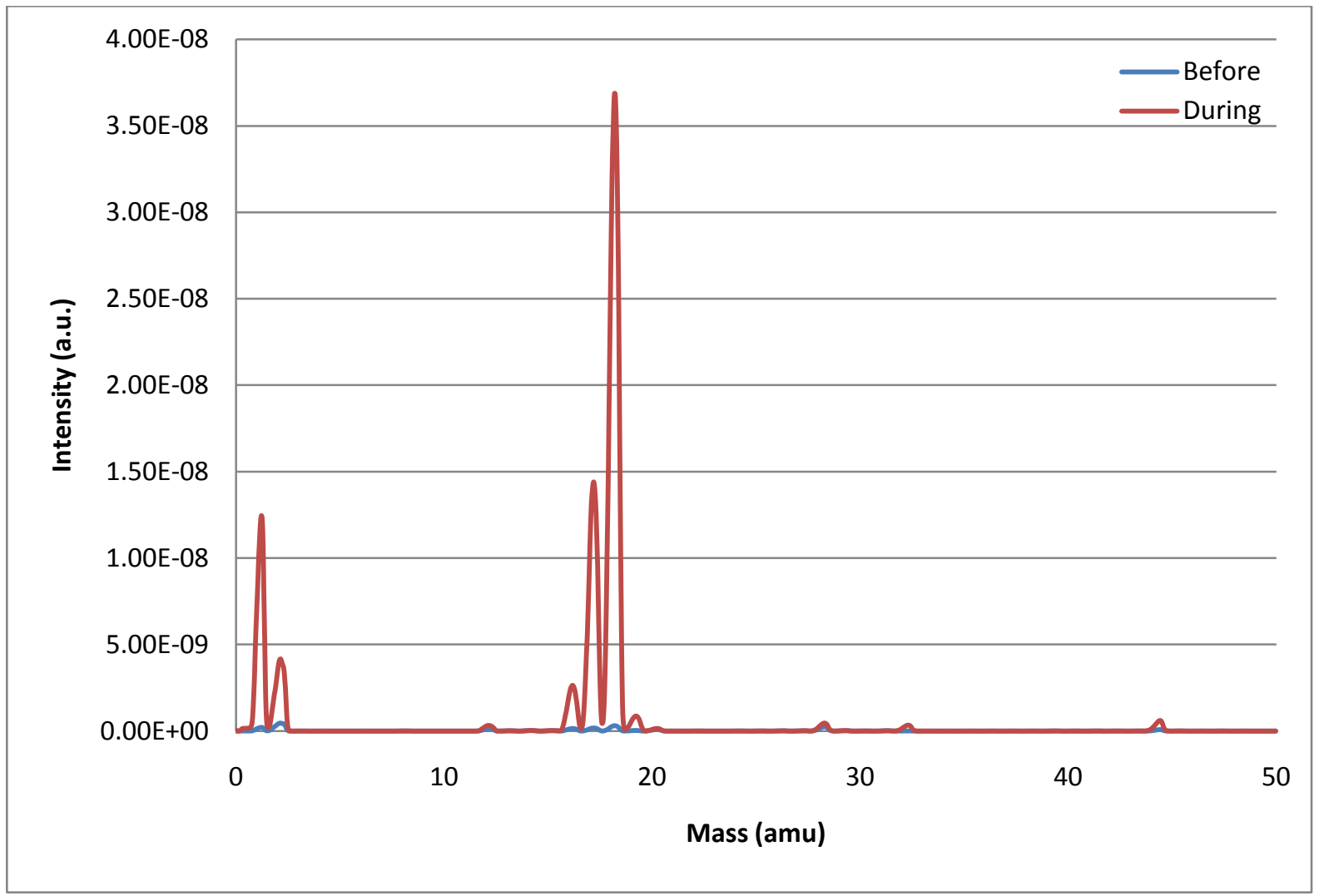

Figure 5.94: Residual gas spectrum of the main chamber before and during gas injection.

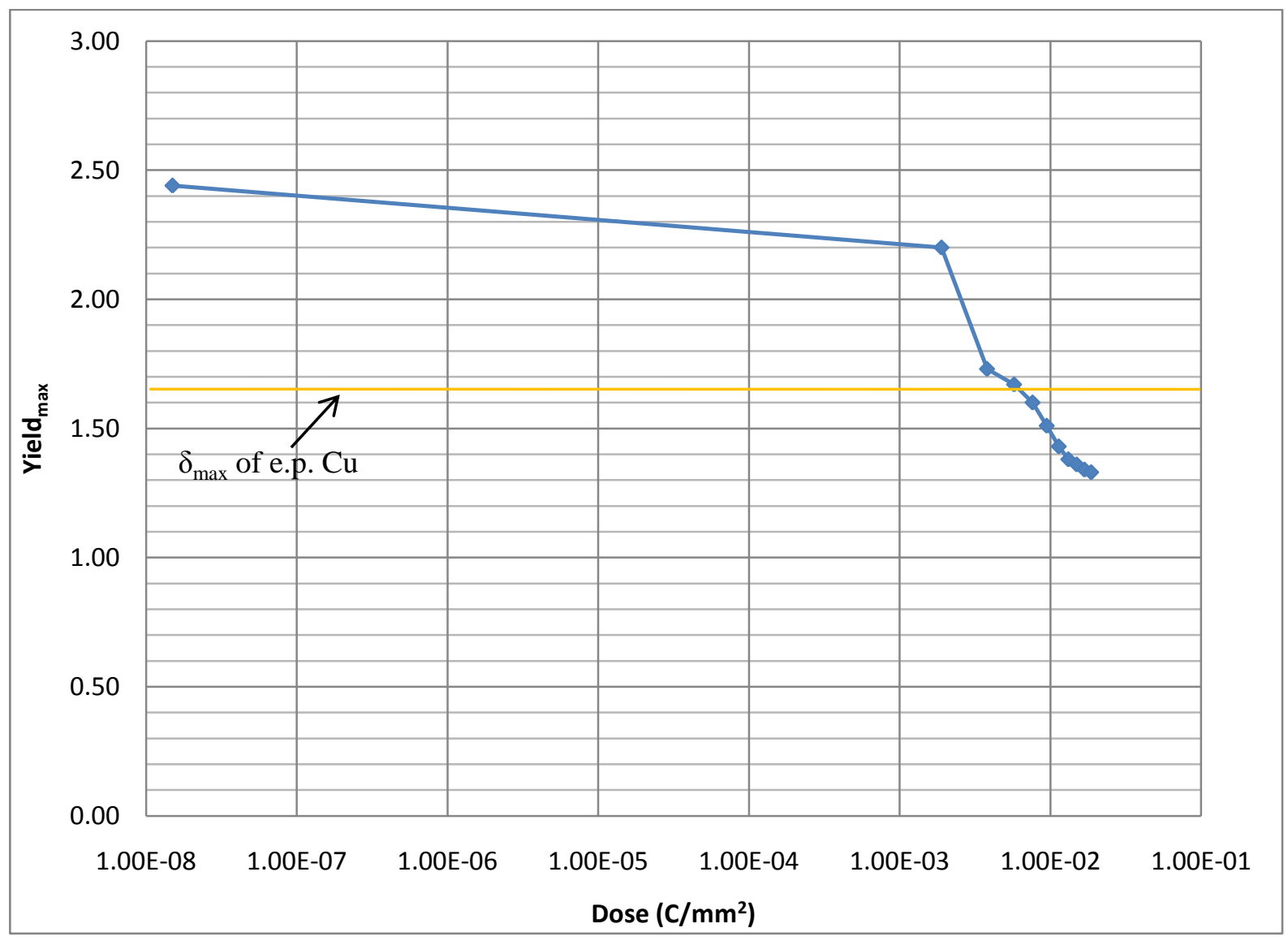

Figure 5.95: Maximum yield as a function of electron dose on 25ML $\mathrm{H}_{2} \mathrm{O} / \mathrm{Cu}$. 


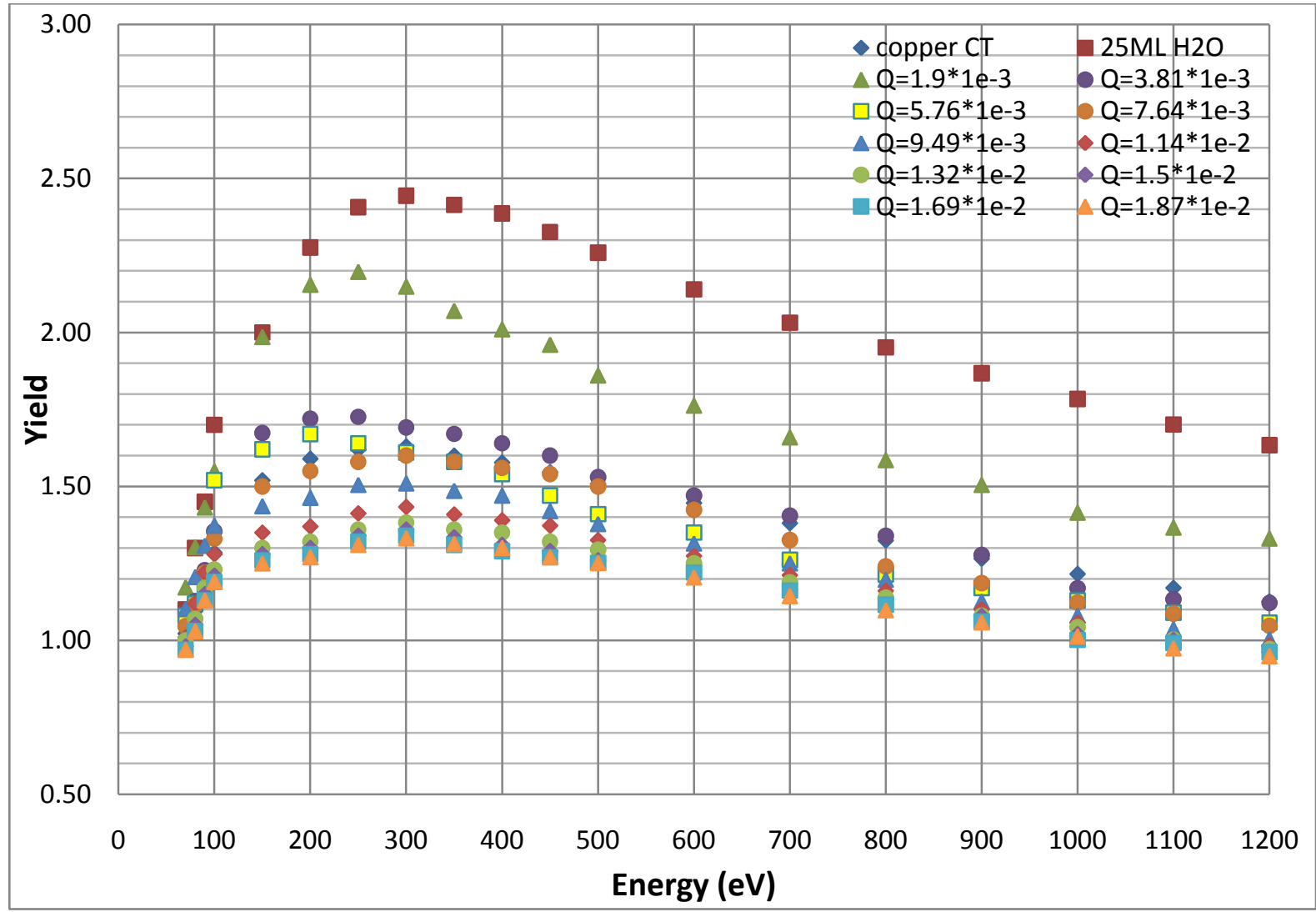

Figure 5.96: Secondary electron yield of water during conditioning.

Curve 1: Secondary electron emission of electro-polished copper at 4,7K without any water adsorption. The beam current for primary electrons with an energy of $300 \mathrm{eV}$ was $3.5 \cdot 10^{-8} \mathrm{~A}$. The total dose during measurement was $1.98 \cdot 10^{-8} \mathrm{C} / \mathrm{mm} 2$. Curve 2: Secondary electron emission of $25 \mathrm{ML}$ of water physisorbed on electro polished copper. The beam current for primary electrons with a kinetic energy of $300 \mathrm{eV}$ was $3.5 \cdot 10^{-8} \mathrm{~A}$. The total dose during measurement was $1.98 \cdot 10^{-8} \mathrm{C} / \mathrm{mm} 2$. Curve 3: First curve with conditioning of $25 \mathrm{ML}$ water on copper. Total dose was of $1.9 \cdot 10^{-3} \mathrm{C} / \mathrm{mm} 2$. Curve 4: Second conditioning. The total dose was $3.81 \cdot 10^{-3} \mathrm{C} / \mathrm{mm} 2$. Curve 5: Third conditioning. The total dose was $5.76 \cdot 10^{-3} \mathrm{C} / \mathrm{mm} 2$. Curve 6: Fourth conditioning. The total dose was $7.64 \cdot 10^{-3} \mathrm{C} / \mathrm{mm} 2$. Curve 7: Fifth conditioning. The total dose was $9.49 \cdot 10^{-3} \mathrm{C} / \mathrm{mm} 2$. Curve 8: Sixth conditioning. The total dose was $1.14 \cdot 10^{-2} \mathrm{C} / \mathrm{mm} 2$. Curve 9: Seventh conditioning. The total dose was $1.32 \cdot 10^{-2} \mathrm{C} / \mathrm{mm} 2$. Curve 10: Eight conditioning. The total dose was $1.5 \cdot 10^{-2} \mathrm{C} / \mathrm{mm} 2$. Curve 11: Ninth conditioning. The total dose was $1.69 \cdot 10^{-2} \mathrm{C} / \mathrm{mm} 2$. Curve 12: Tenth conditioning. The total dose was $1.875 \cdot 10^{-2} \mathrm{C} / \mathrm{mm} 2$.

During the first 3 electron bombardments we desorb the adsorbed water, therefore the yield decreases. After desorbing all the water we start doing conditioning on the electro polished copper sample.

Figure 5.97 shows the energy of the primary electrons at maximum yield as a function of the dose. Without conditioning the maximum is at $300 \mathrm{eV}$, while conditioning the water the energy 
for the maximum yield decreases and reaches $200 \mathrm{eV}$. With starting conditioning of copper, the primary energy at the maximum yield goes up again to $300 \mathrm{eV}$.

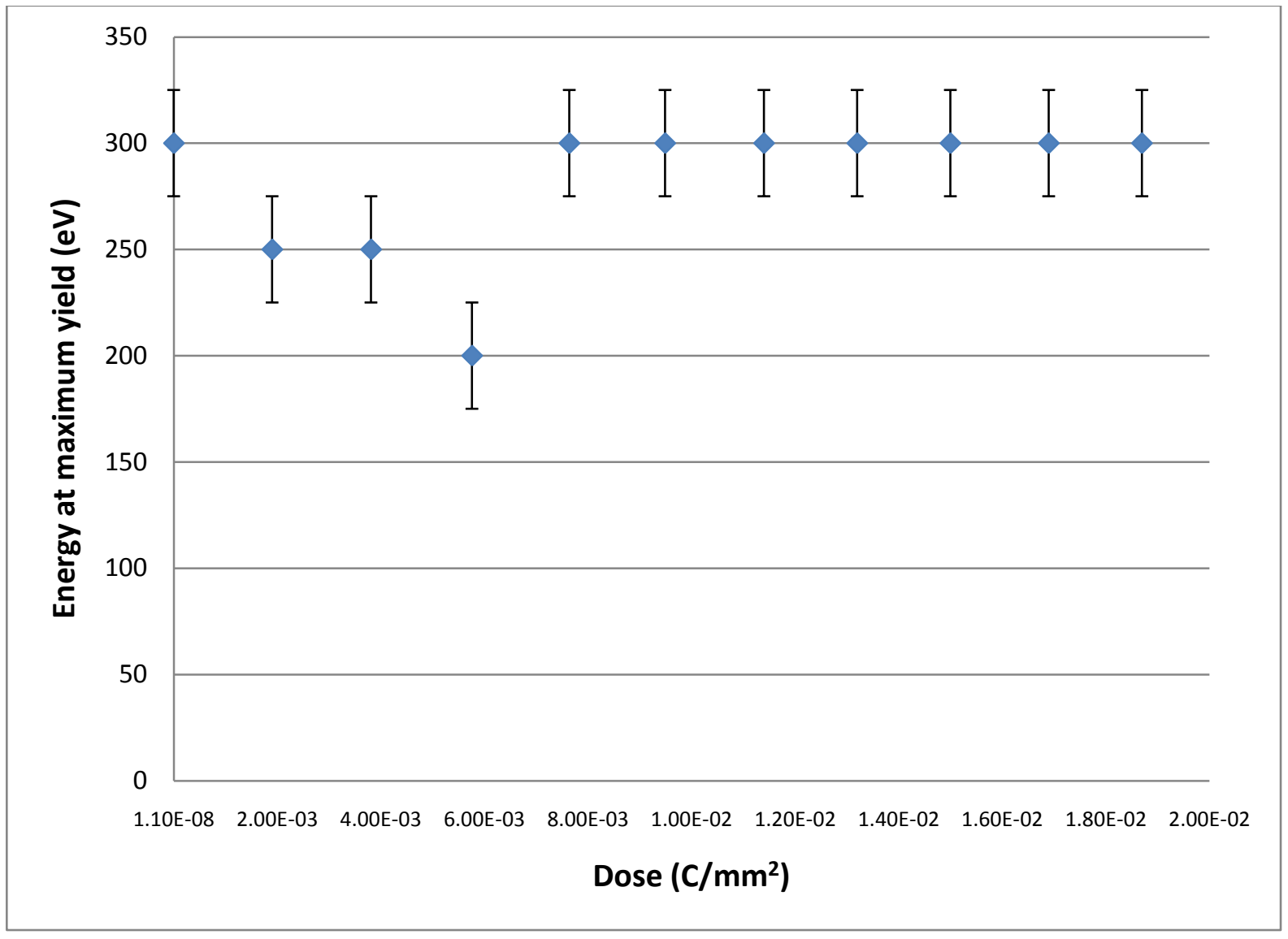

Figure 5.97: Energy at maximum yield as a function of the Dose of $\mathrm{H}_{2} \mathrm{O}$.

Before doing the conditioning of carbon monoxide, the sample has been changed, because the previous sample was already conditioned. A new electro-polished copper with a maximum yield of 1.64 at cryogenic temperatures has been mounted. After cooling down the sample, 25 monolayers of carbon monoxide are physisorbed on the sample. Again the SEY measurements have been performed with a pulsed beam current of $3.5 \cdot 10^{-8} \mathrm{~A}$ (electron gun filament of 2.2A). For conditioning (the filament current has been increased to $2.4 \mathrm{~A}$ and therefore) the beam current was increased to $1.5 \cdot 10^{-5} \mathrm{~A}$. The electron beam was not pulsed during conditioning. Electrons with energy of $300 \mathrm{eV}$ have been used. The pressure in the vacuum chamber during conditioning was around $1.2 \cdot 10^{-8} \mathrm{mbar}$. Figure 5.98 shows the yield as a function of energy during the conditioning process. Figure 5.99 shows the maximum yield as a function of electron dose. 


\section{Chapter 5-Results}

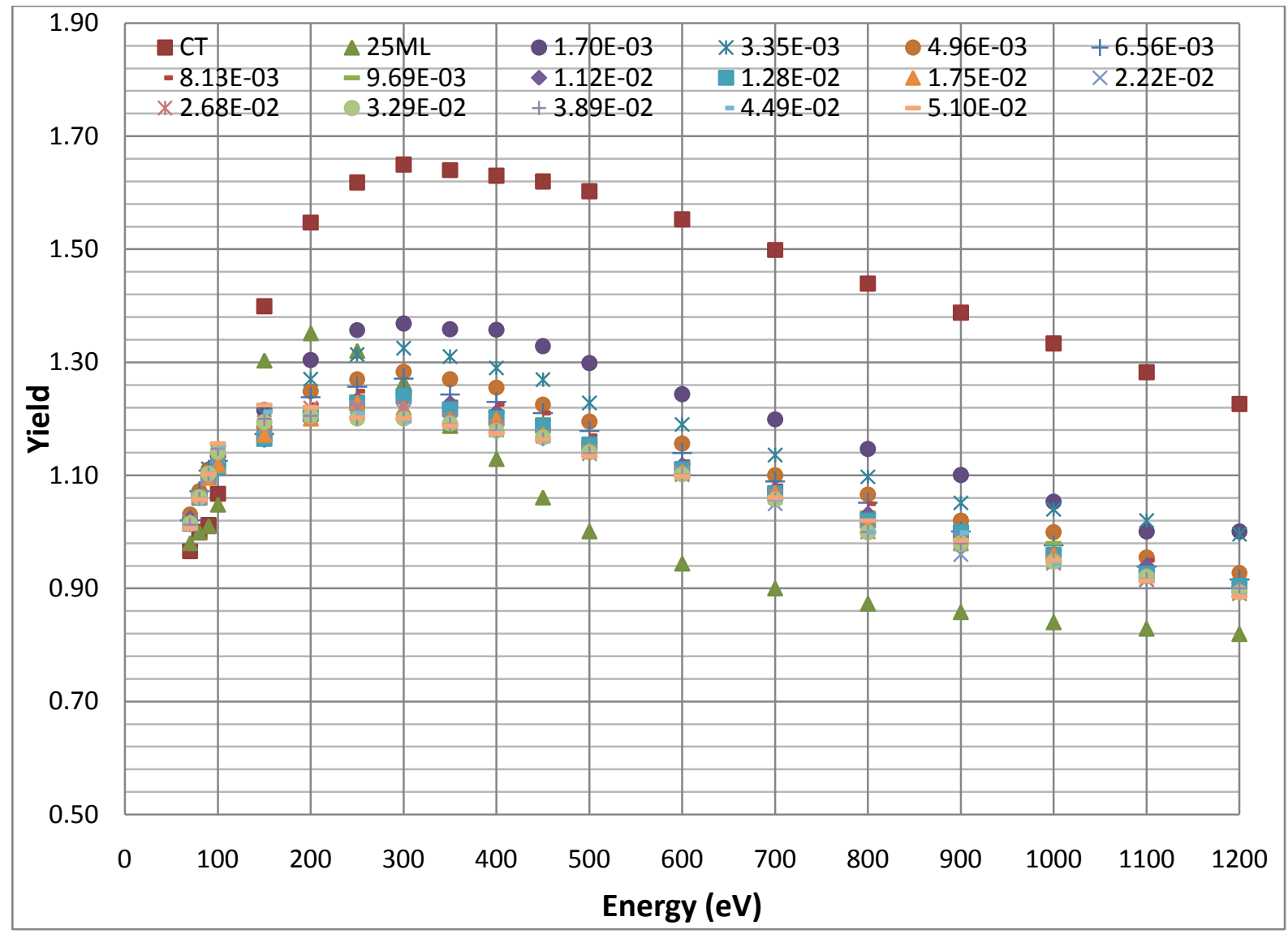

Figure 5.98: Secondary electron yield of carbon monoxide during conditioning.

Curve 1: Secondary electron emission of electro-polished copper at 4,7K without any carbon monoxide adsorption. The beam current for primary electrons with an energy of $300 \mathrm{eV}$ was $3.5 \cdot 10^{-8} \mathrm{~A}$. The total dose during measurement was $1.98 \cdot 10^{-8} \mathrm{C} / \mathrm{mm}^{2}$. Curve 2: Secondary electron emission of $25 \mathrm{ML}$ of carbon monoxide physisorbed on electro-polished copper. The beam current for primary electrons with a kinetic energy of $300 \mathrm{eV}$ was $3.5 \cdot 10^{-8} \mathrm{~A}$. The total dose during measurement was $1.98 \cdot 10^{-8} \mathrm{C} / \mathrm{mm}^{2}$. Curve 3: First curve with conditioning of $25 \mathrm{ML}$ carbon monoxide on copper. Total dose was of $1.7 \cdot 10^{-3} \mathrm{C} / \mathrm{mm}^{2}$. Curve 4: Second conditioning. The total dose was $3.35 \cdot 10^{-3} \mathrm{C} / \mathrm{mm}^{2}$. Curve 5: Third conditioning. The total dose was $4.96 \cdot 10^{-3} \mathrm{C} / \mathrm{mm}^{2}$. Curve 6: Fourth conditioning. The total dose was $6.56 \cdot 10^{-3} \mathrm{C} / \mathrm{mm}^{2}$. Curve 7: Fifth conditioning. The total dose was $8.13 \cdot 10^{-3} \mathrm{C} / \mathrm{mm}^{2}$. Curve 8: Sixth conditioning. The total dose was $9.69 \cdot 10^{-3} \mathrm{C} / \mathrm{mm}^{2}$. Curve 9: Seventh conditioning. The total dose was $1.12 \cdot 10^{-2} \mathrm{C} / \mathrm{mm}^{2}$. Curve 10: Eight conditioning. The total dose was $1.28 \cdot 10^{-2} \mathrm{C} / \mathrm{mm}^{2}$. Curve 11: Ninth conditioning. The total dose was $1.75 \cdot 10^{-2} \mathrm{C} / \mathrm{mm}^{2}$. Curve 12: Tenth conditioning. The total dose was $2.22 \cdot 10^{-2} \mathrm{C} / \mathrm{mm}^{2}$. Curve 13: 11 th conditioning. The total dose was $2.68 \cdot 10^{-2} \mathrm{C} / \mathrm{mm}^{2}$. Curve 14: $12^{\text {th }}$ conditioning. The total dose was $3.29 \cdot 10^{-2} \mathrm{C} / \mathrm{mm}^{2}$. Curve 15: 13 th conditioning. The total dose was $3.89 \cdot 10^{-2} \mathrm{C} / \mathrm{mm}^{2}$. Curve 16: 14 th conditioning. The total dose was $4.49 \cdot 10^{-2} \mathrm{C} / \mathrm{mm}^{2}$. Curve 17: 15 th conditioning. The total dose was $5.10 \cdot 10^{-2} \mathrm{C} / \mathrm{mm}^{2}$. 


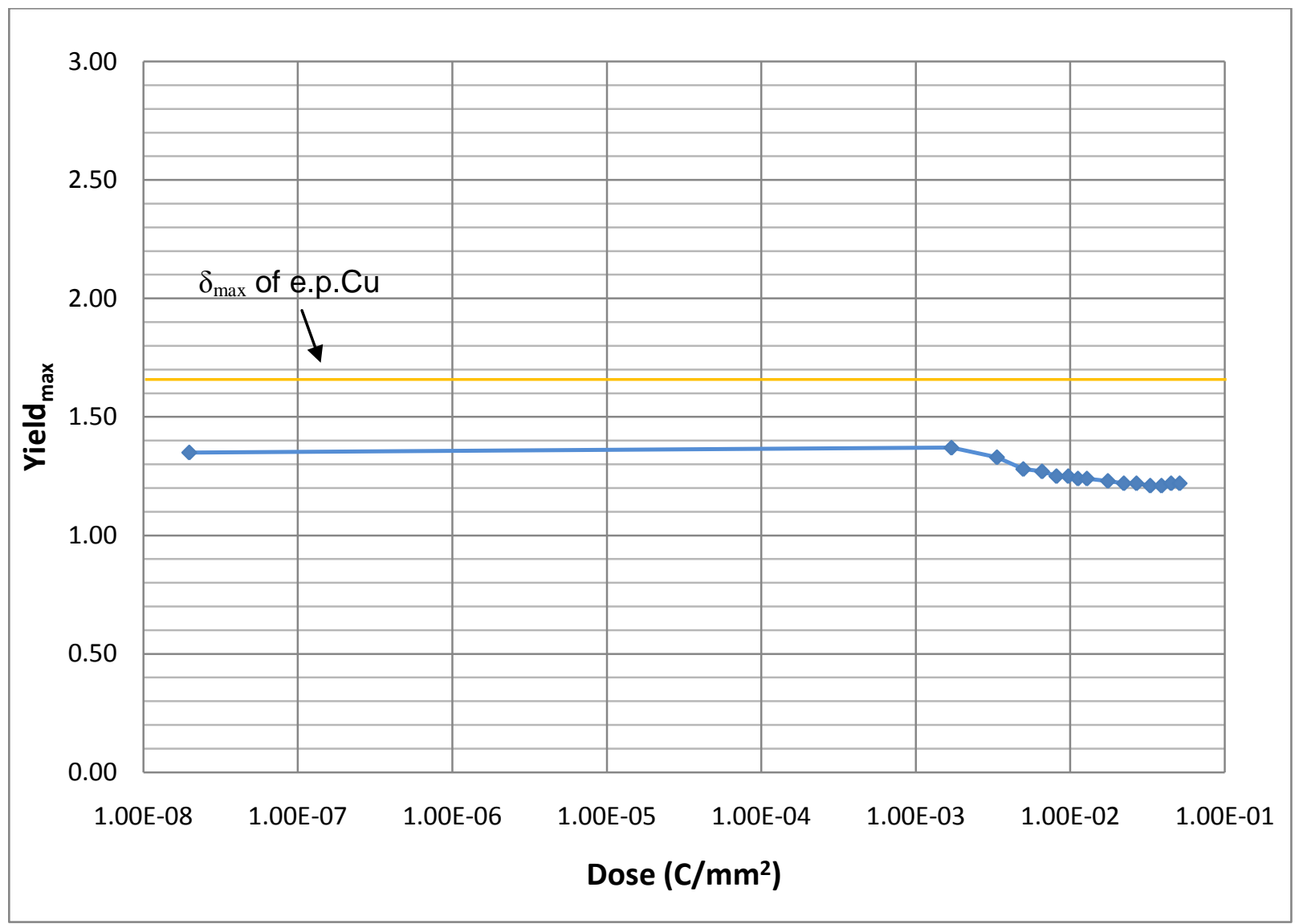

Figure 5.99: Maximum yield as a function of electron dose of CO.

In figure 5.99 it can be observed that with the first conditioning due to desorption of carbon monoxide, the yield increases slightly and then with following conditioning it decreases due to carbon formation [32]. It reaches the saturation with app. 1.21.

Figure 5.100 shows the energy at the maximum yield as a function of the total dose. The energy for the maximum yield of 25 monolayers of carbon monoxide is at $200 \mathrm{eV}$. During conditioning it increases to $300 \mathrm{eV}$ and falls off with increasing dose again back to $200 \mathrm{eV}$. 


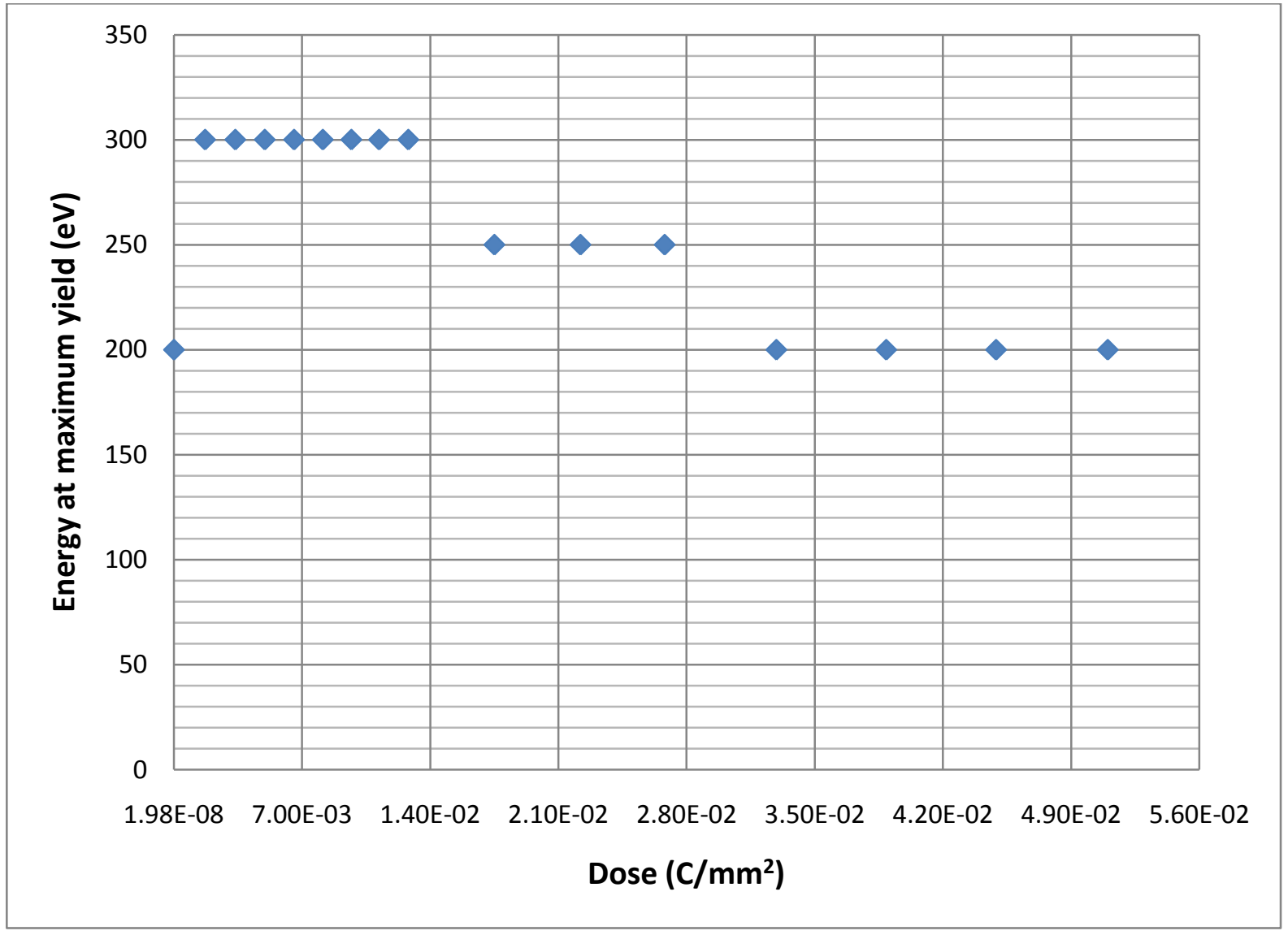

Figure 5.100: Energy at the maximum yield as a function of total electron dose of CO.

26 hours after conditioning with carbon monoxide, 30 monolayers of $\mathrm{N}_{2}$ are physisorbed on the same pre-conditioned electro-polished sample. The maximum yield is 1.45 by energy of $200 \mathrm{eV}$. This value is lower than the normal value for the electro polished copper. The reason is that the sample was already conditioned the day before. It increases 2.01 by $300 \mathrm{eV}$ after physisorption of 30 monolayers $\mathrm{N}_{2}$.

Figure 5.101 shows the yield as a function of energy during conditioning process.

Figure 5.102 shows the maximum yield as a function of the total electron dose.

Figure 5.103 shows the energy at maximum yield as a function of total dose.

It is possible to see that the maximum yield of $\mathrm{N}_{2}$ falls off to 1.56 after the first conditioning dose and the energy for the maximum yield decreases from $300 \mathrm{eV}$ down to $250 \mathrm{eV}$. 


\section{Chapter 5-Results}

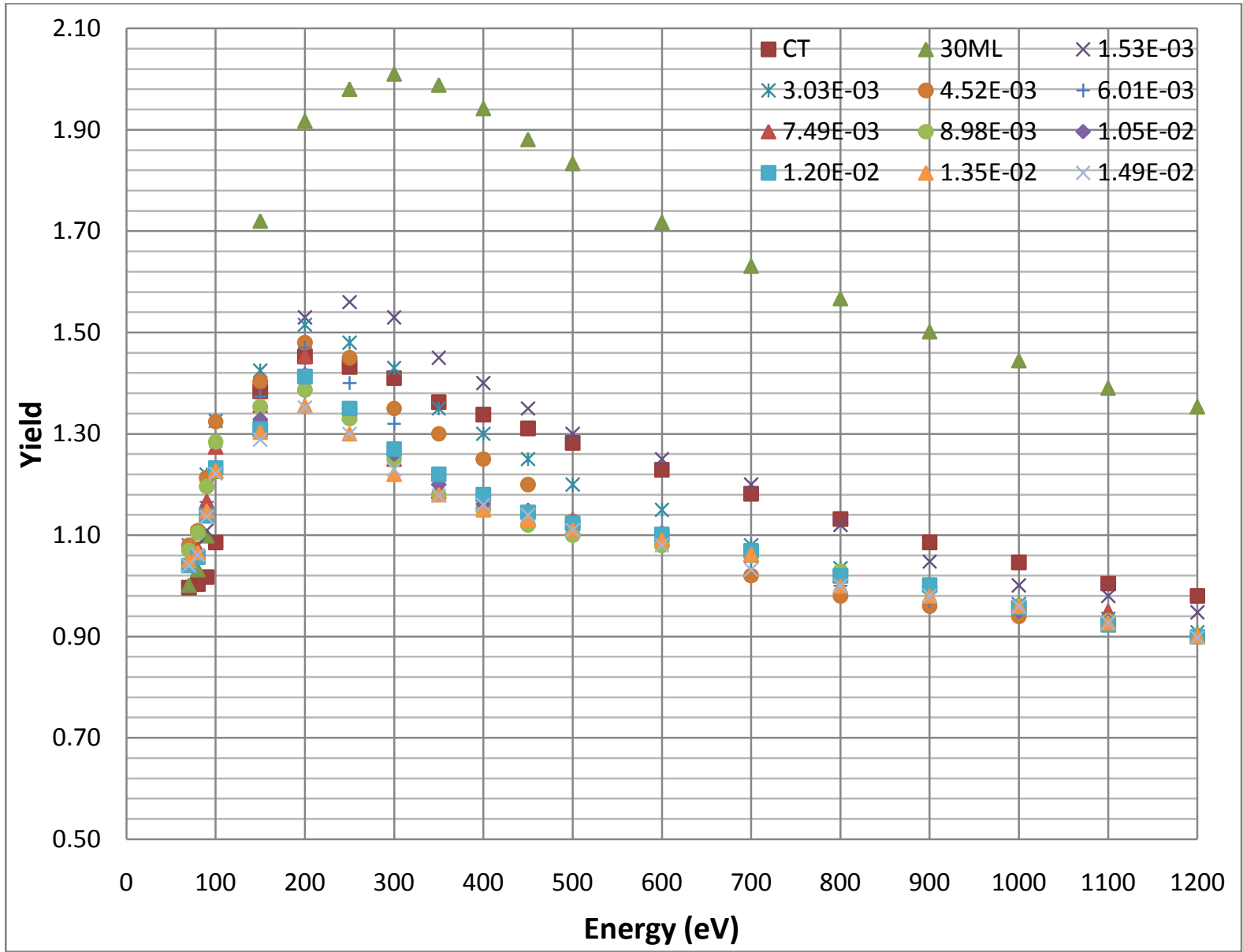

Figure 5.101: Secondary electron yield of $N_{2}$ during conditioning.

Curve 1: Secondary electron emission of electro-polished copper at 4,7K without any $N_{2}$ adsorption. The beam current for primary electrons with an energy of $300 \mathrm{eV}$ was $3.5 \cdot 10^{-8} \mathrm{~A}$. The total dose during measurement was $1.98 \cdot 10^{-8} \mathrm{C} / \mathrm{mm}^{2}$. Curve 2: Secondary electron emission of $30 \mathrm{ML}$ of $\mathrm{N}_{2}$ physisorbed on electro polished copper. The beam current for primary electrons with a kinetic energy of $300 \mathrm{eV}$ was $3.5 \cdot 10^{-8} \mathrm{~A}$. The total dose during measurement was $1.98 \cdot 10^{-8} \mathrm{C} / \mathrm{mm}^{2}$. Curve 3: First curve with conditioning of $30 \mathrm{ML} \mathrm{N}_{2}$ on copper. Total dose was of $1.53 \cdot 10^{-3} \mathrm{C} / \mathrm{mm}^{2}$. Curve 4: Second conditioning. The total dose was $3.03 \cdot 10^{-3} \mathrm{C} / \mathrm{mm}^{2}$. Curve 5: Third conditioning. The total dose was $4.52 \cdot 10^{-3} \mathrm{C} / \mathrm{mm}^{2}$. Curve 6: Fourth conditioning. The total dose was $6.01 \cdot 10^{-3} \mathrm{C} / \mathrm{mm}^{2}$. Curve 7: Fifth conditioning. The total dose was $7.4910^{-3} \mathrm{C} / \mathrm{mm}^{2}$. Curve 8: Sixth conditioning. The total dose was $8.98 \cdot 10^{-3} \mathrm{C} / \mathrm{mm}^{2}$. Curve 9: Seventh conditioning. The total dose was $1.05 \cdot 10^{-2} \mathrm{C} / \mathrm{mm}^{2}$. Curve 10: Eight conditioning. The total dose was $1.20 \cdot 10^{-2} \mathrm{C} / \mathrm{mm}^{2}$. Curve 11: Ninth conditioning. The total dose was $1.35 \cdot 10^{-2} \mathrm{C} / \mathrm{mm}^{2}$. Curve 12: Tenth conditioning. The total dose was $1.49 \cdot 10^{-2} \mathrm{C} / \mathrm{mm}^{2}$. 


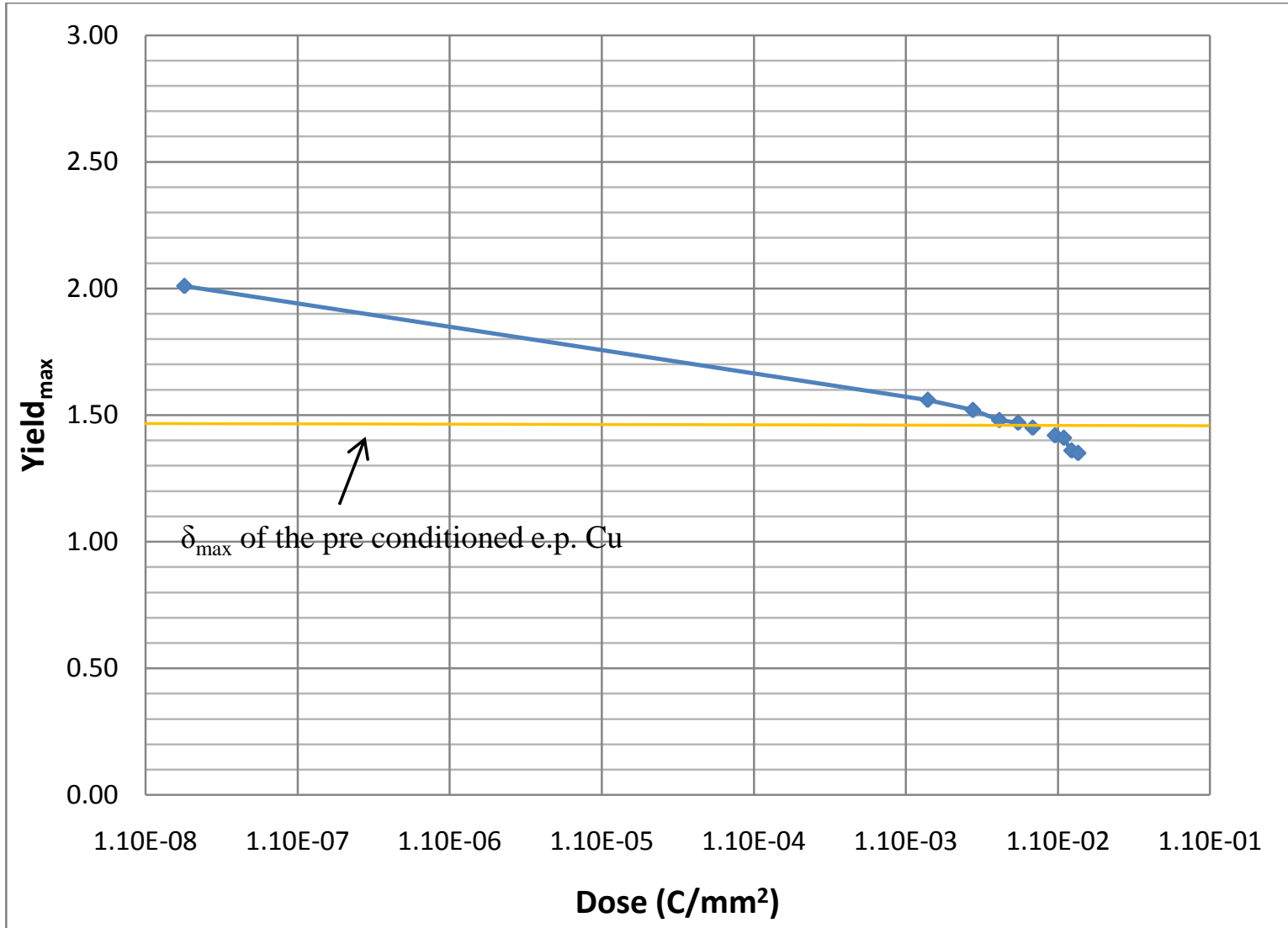

Figure 5.102: Maximum yield as a function of total electron dose of $\mathrm{N}_{2}$.

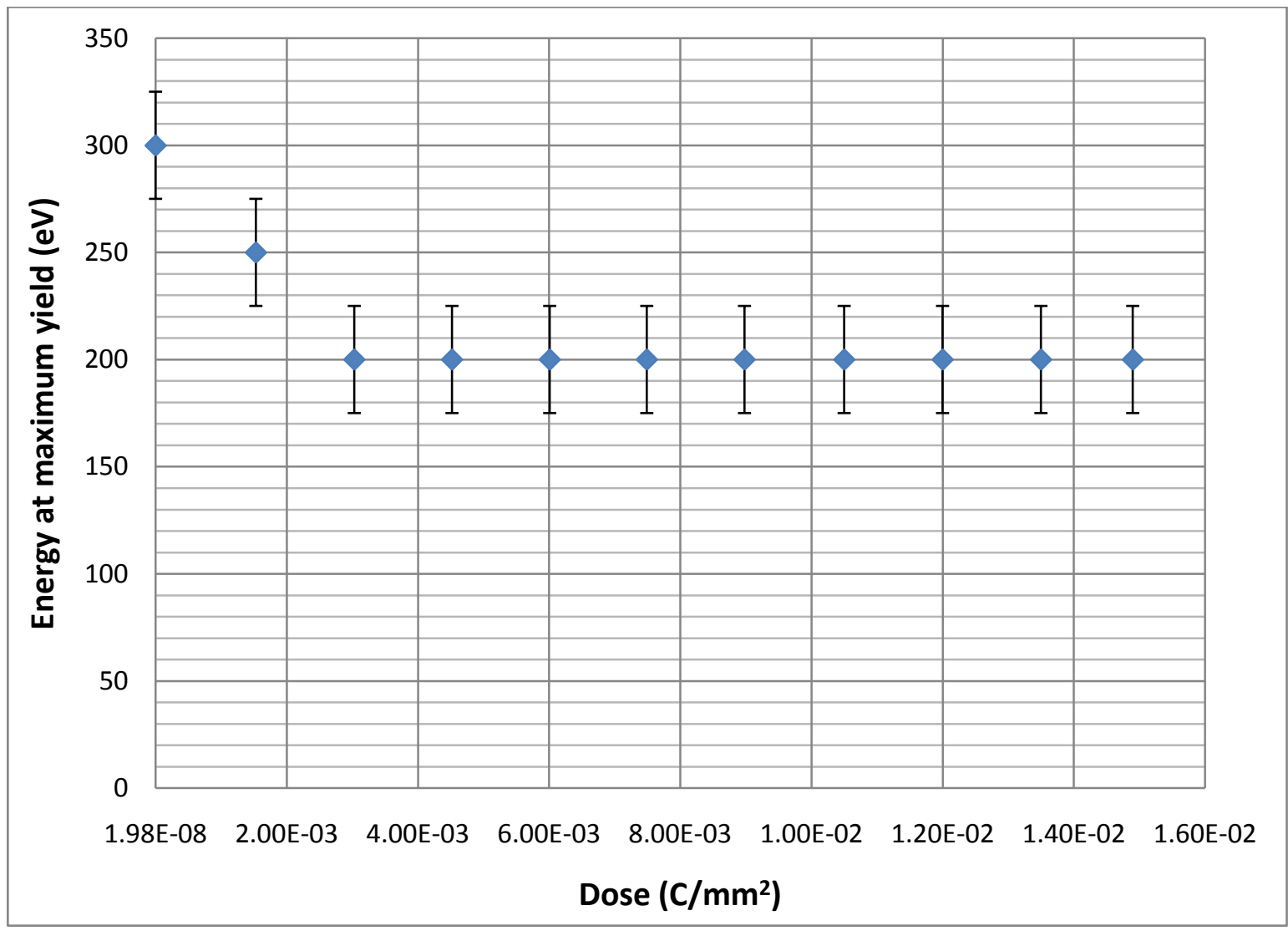

Figure 5.103: Energy at maximum yield as a function of total electron dose of $\mathrm{N}_{2}$. 


\section{Discussion}

\subsection{Secondary electron yield}

\subsubsection{Tentative interpretation of the coverage dependence of SEY}

Secondary electron generation in the presence of adsorbate layers can occur in the substrate material and/or in the adsorbate layer. Which part has the dominant effect in the generation depends on the thickness of the adsorbate layer. Therefore it is easier to divide the discussion into three parts, in order to give an explanation for the common points of different adsorbed gases on different substrates (Figure 6.1). The first part is about thin layers (range of the primary electrons > thickness of the adsorbate), where the substrate and the adsorbate/substrate interface are more important than the adsorbate on the secondary electron generation. The second part is the intermediate thickness, where the adsorbate starts to get more and more important and the substrate and the adsorbate/substrate interface start to lose their importance. The third part is about the thick layers (range of the primary electrons < thickness of the adsorbate). Here the substrate and the adsorbate/substrate interface lose their importance completely. The adsorbate is the bulk specimen and it is the only factor which contributes in the total number of secondary electrons. 


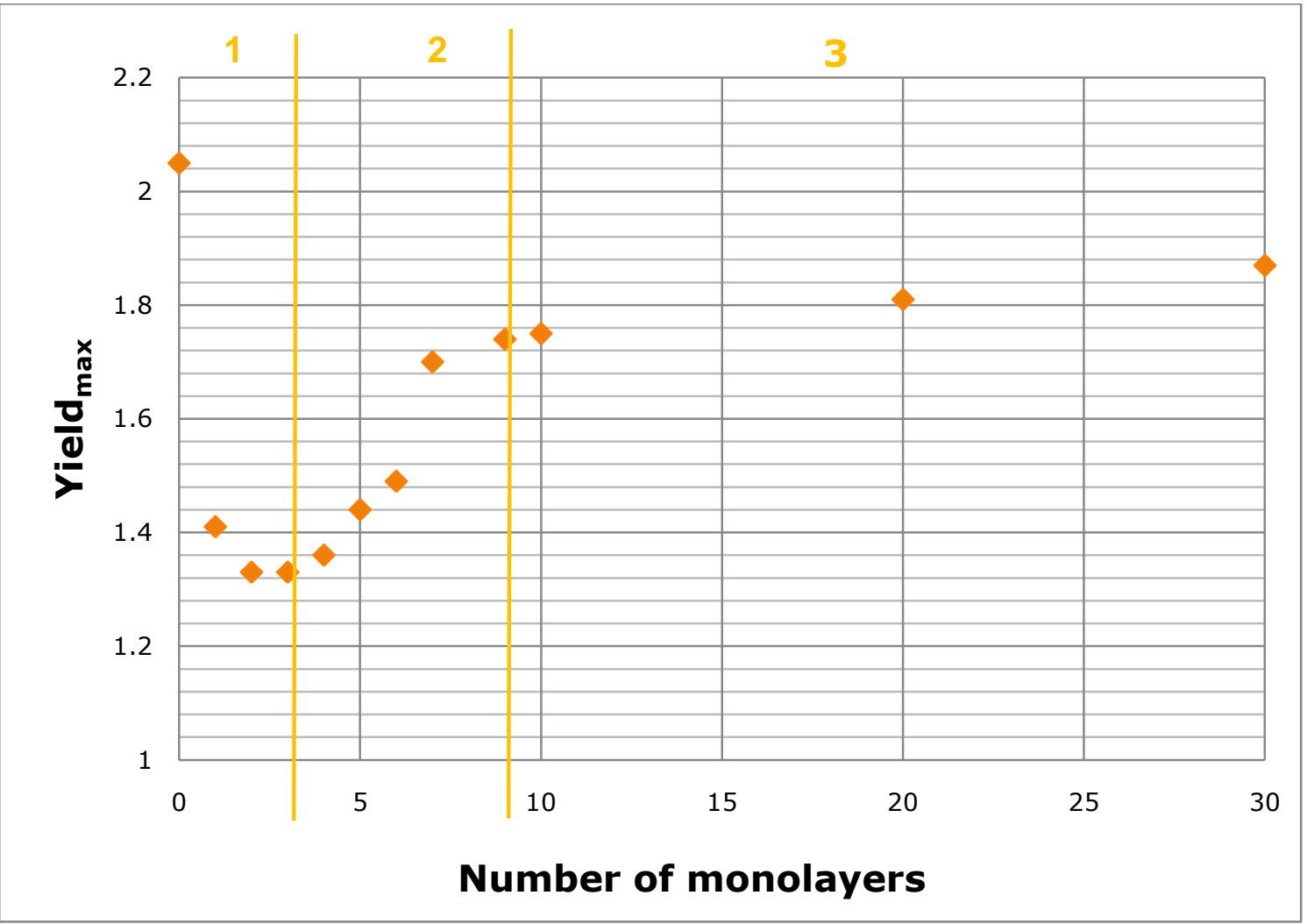

Figure 6.1: Three parts of the curve for the maximum yield as a function of the number of monolayers.

In thin layers, a big part of the primary electrons enter the substrate material where they produce secondary electrons. Not all the secondary electrons generated in the substrate can reach the adsorbate/vacuum interface. Some of the secondary electrons may experience scattering at the dielectric/substrate interface and therefore be attenuated. This means, the presence of the adsorbates causes a decrease in the contribution of the substrate material in the total number of secondary electrons. Interface contribution becomes important only at low thickness.

With increasing distance $d$ to the metal surface, the primary electrons start to generate secondary electrons in the adsorbate layers as well as in the substrate metal. Once reaching thick layers, where the primary electrons cannot penetrate anymore in the metal substrate, the adsorbate layer is the only contribution to the secondary electron generation. So the measured secondary electron yield is the yield of the adsorbate only.

To compare with the experiment, we can have a look at the curves (Figure 5.84, 5.85, 5.86 and 5.87), where the maximum yield of a physisorbed gas is shown for 3 different substrates as a function of the number of monolayers: 
For few layers (the first 3-4 monolayers), the maximum yield for each substrate is varying. The total yield of the substrate together with the adsorbate is lower than the yield of the substrate only, because of the attenuation of the secondary electrons.

By increasing the number of adsorbate layers, the value for the maximum secondary electron yield starts to get independent of the substrate. This is the intermediate thickness explained above. If the adsorbate has a lower yield than the substrate as in the case of $\mathrm{CO}$, $\mathrm{CH}_{4}$ and $\mathrm{C}_{2} \mathrm{H}_{6}$, the yield continues to decrease. If the adsorbate has a higher yield than the substrate as in case of $\mathrm{N}_{2}$ or $\mathrm{CO}_{2}$ the yield starts to increase.

By thick layers, the value for the maximum yield is the same for each substrate material. This is the region where the substrate loses its importance completely and the adsorbate is the only source of the secondary electrons. Therefore the maximum yield of each physisorbed gas doesn't vary for different substrates.

\subsubsection{Possible explanations of SEY of "bulk" adsorbate}

When the range of the primary electrons is less than the thickness of the condensed layers, the adsorbate can be considered as the "bulk". Different adsorbates have different secondary electron yields. To explain the different absolute SEY for different adsorbates, it is important to investigate the generation of the secondary electrons in insulators, their transport towards the vacuum and their escape. According to the present empirical models $[30,48-50]$ the following quantities are relevant for the excitation and generation of secondary electrons:

- Average energy required to create an electron hole pair $\mathrm{E}(\mathrm{eh}) \propto$ band gap

- Stopping power $\alpha$ number of electrons $Z$, density of solid $\rho$

- Range of primary electrons $\alpha$ density of solid $\rho$

\section{Average energy required to create an electron hole pair $E(e h):$}

In insulators, the generation of the secondary electrons occur via excitation of valence electrons into the conduction band as shown in Figure 6.2. This phenomenon requires an average energy $E(e h)$ to create electron hole pairs. The lower the required energy $E(e h)$, the more electron hole pairs can be generated for the same impinging primary energy and the higher the SEY will be. This is true under the assumption that the entire energy of the primaries is used to generate secondary electrons. There are some experimental data about $\mathrm{E}(\mathrm{eh})$ for rare gases. $\mathrm{E}(\mathrm{eh})$ for $\mathrm{Ar}$ is $27 \pm 1 \mathrm{eV}$, for $\mathrm{Kr} 25 \pm 1 \mathrm{eV}$ and for $\mathrm{Xe} 24 \pm 1 \mathrm{eV}$. According to this consideration one expects that the SEY increases with decreasing $E(e h)$. The 
differences are small but comparing physisorbed layers of such gases at similar monolayer coverage the SEY follows indeed the expected behaviour [30].

As an example in the model of Alig and Bloom [48], the average energy to create an electron hole pair is 2.8 times the band gap. The model is developed for semiconductors, therefore the calculated and the experimental values may differ (Table 6.1).

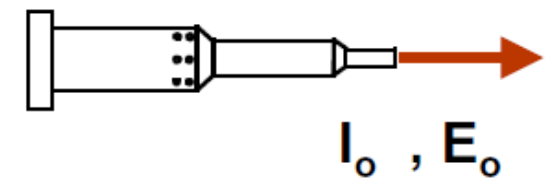

INJECTION

Impacting e- beam

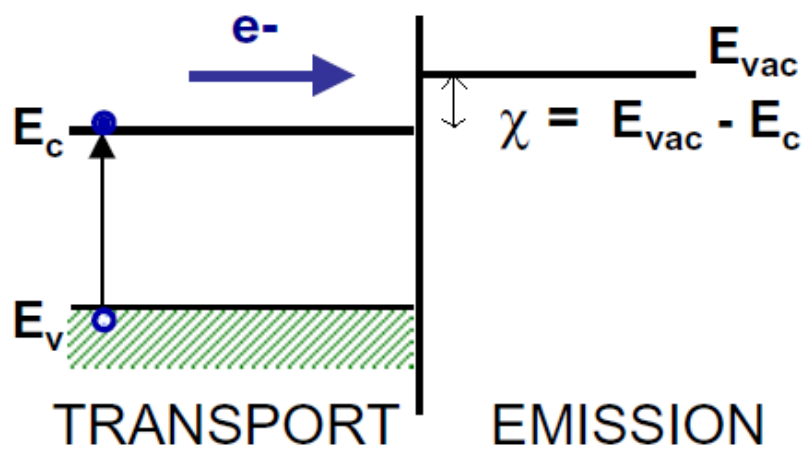

EMISSION

Figure 6.2: Generation of secondary electrons in insulators via electron beam coming from an electron gun. $\chi$ is the electron affinity and it is either very low or even negative in noble gases.

\begin{tabular}{|c|c|c|c|c|}
\hline Material & Band Gap (eV) & $\delta_{\max }$ & $\begin{array}{c}E(e h) \text { exp. } \\
(\mathbf{e V})\end{array}$ & $\begin{array}{c}\text { E(eh) cal. } \\
(\mathbf{e V})\end{array}$ \\
\hline $\mathrm{CO}$ & 1.62 & 1.25 & - & 4.5 \\
\hline $\mathrm{CH}_{4}$ & 1.75 & 1.47 & - & 4.9 \\
\hline $\mathrm{C}_{2} \mathrm{H}_{6}$ & - & 1.61 & - & - \\
\hline $\mathrm{CO}_{2}$ & 10 & 2 & - & 28 \\
\hline $\mathrm{N}_{2}$ & 12 & 1.87 & - & 33.6 \\
\hline $\mathrm{Ne}$ & 21.69 & 5.25 & - & 60.7 \\
\hline $\mathrm{Ar}$ & 14.15 & 9.2 & $27 \pm 1$ & 39.6 \\
\hline $\mathrm{Kr}$ & 11.6 & 10.5 & $25 \pm 1$ & 32.5 \\
\hline $\mathrm{Xe}$ & 9.3 & 12 & $24 \pm 1$ & 26 \\
\hline
\end{tabular}

Table 6.1: Band gap of different adsorbates and their maximum SEY. The values for the maximum yield for all the gases but rare gases are the saturation values. The maximum yield of the rare gases is given for 60 monolayers. The saturation value for them is not known.

It is clear that the results for electron hole pair creation energy and SEY correlate. Ne with the highest required energy has the lowest SEY. On the other hand, the model doesn't fit for the other gases. $\mathrm{CO}$ with the lowest required energy has also the lowest yield. This brings us to the point that the band gap is not just affecting the electron hole pair creation energy. It has also other important effects, which changes the SEY. The other effect will be discussed below with the inelastic mean free path. 


\section{Stopping power:}

Stopping power is a function of the number of electrons $Z$ and the density $\rho$ [49]. If the stopping power is large, more secondary electrons can be excited since the energy is dissipated in a shallower depth. Therefore adsorbates with higher stopping powers are expected to have higher yields. The values of stopping power are generally available in a higher energy range than the one used for the SEY measurements in the present work. Therefore a comparison of the stopping power of different adsorbates has been done for $100 \mathrm{keV}$ electrons (See figure 6.3) [49]. With increasing stopping power, the SEY is increasing. Carbon monoxide has almost the same stopping power as $\mathrm{N}_{2}$ but much lower yield than $\mathrm{N}_{2}$ and is not following the general trend. To make a comparison for low energy electrons is difficult, because at low energies the stopping power starts to depend on the details of the density of states.

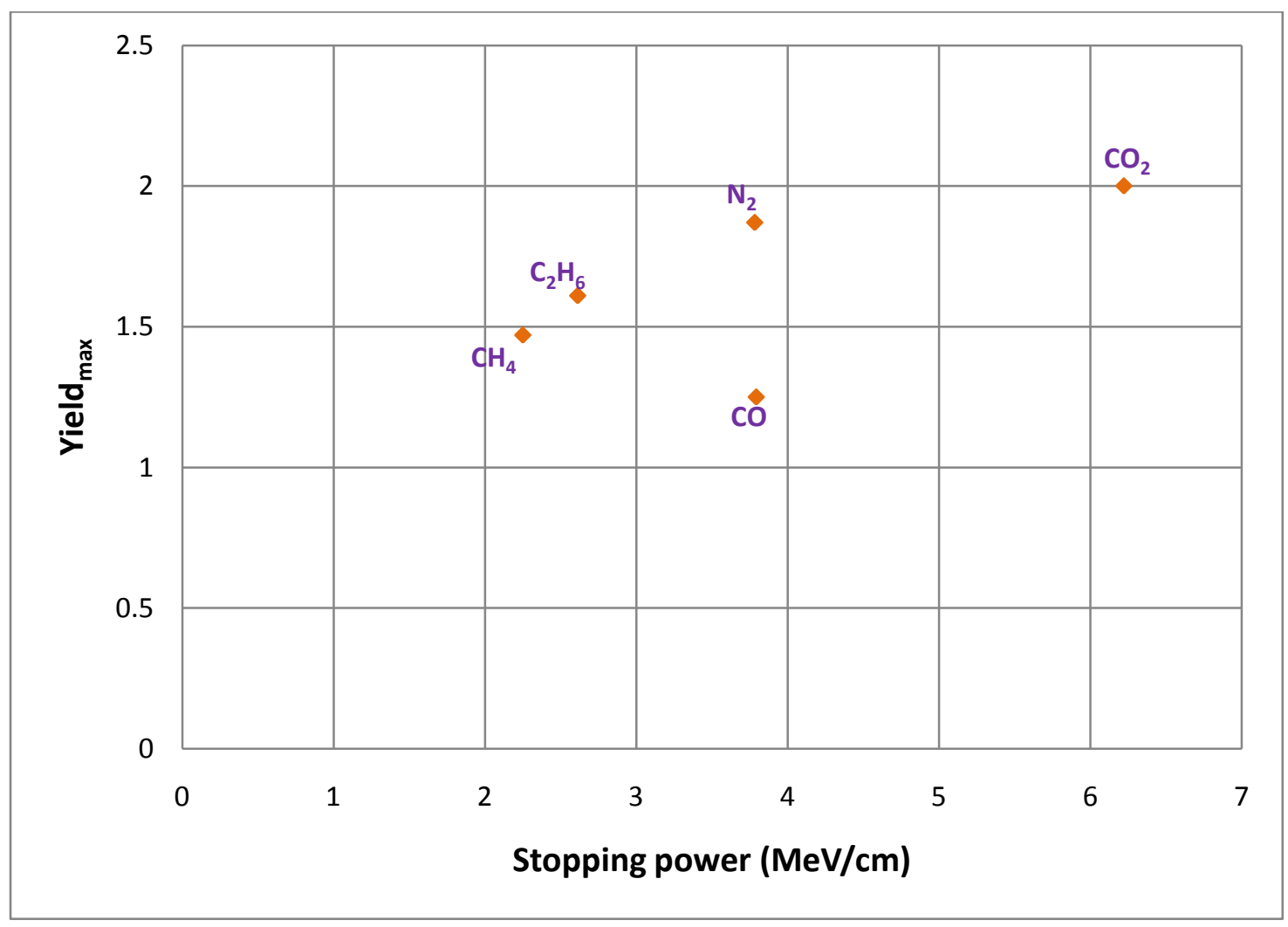

Figure 6.3: Maximum yield as a function of the stopping power.

In figure 6.4 the numbers of electrons per atom for $\mathrm{Kr}, \mathrm{CO}_{2}, \mathrm{~N}_{2}, \mathrm{C}_{2} \mathrm{H}_{6}, \mathrm{CH}_{4}$ and $\mathrm{CO}$ are compared. It is possible to see that with increasing number of electrons/atom, the SEY is increasing. But $\mathrm{CO}$ is not following the behaviour of the other gases. $\mathrm{CO}$ has the lowest yield of all of them but the number of electrons/atom is the same as for $\mathrm{N}_{2}$. 


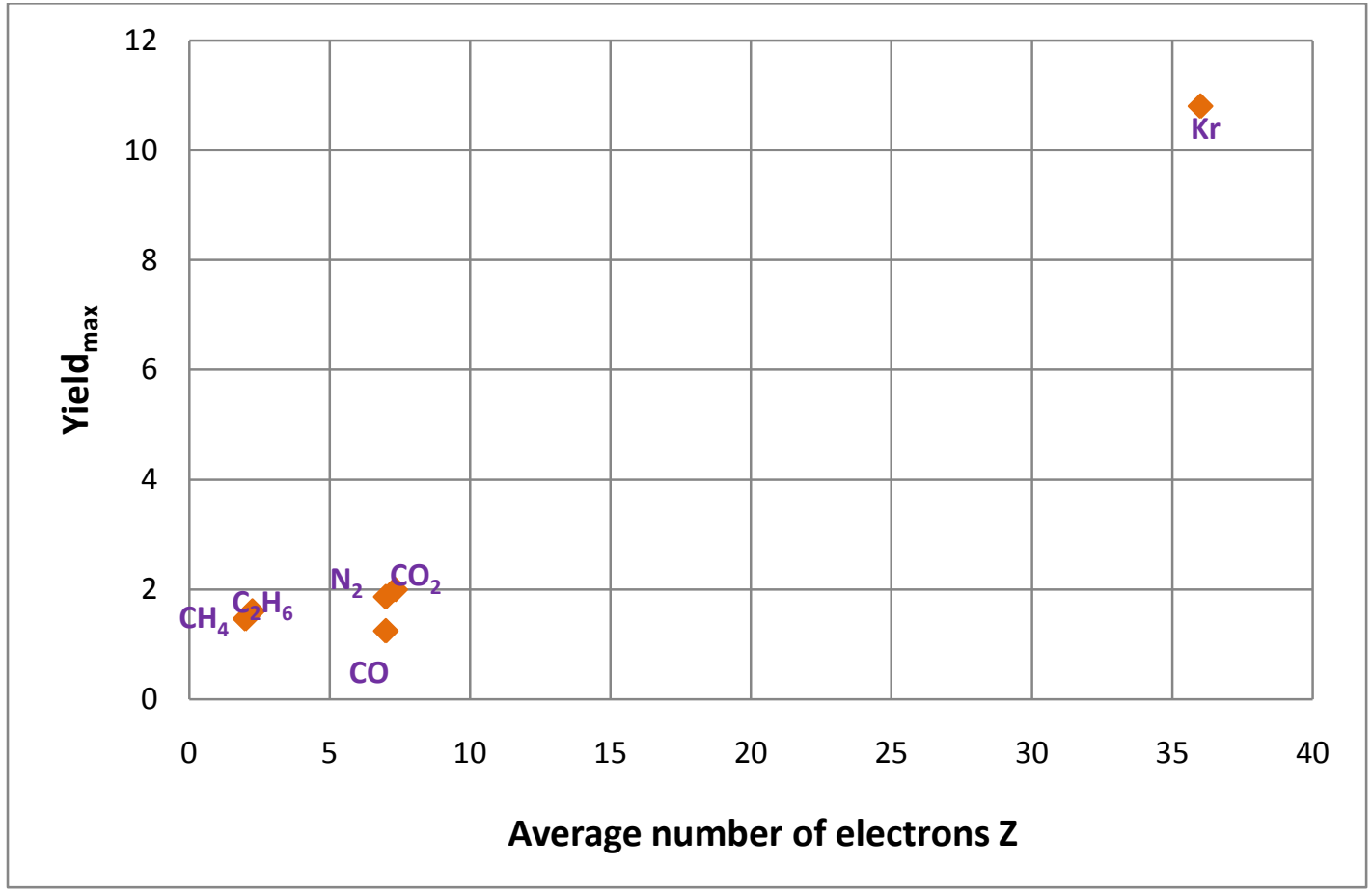

Figure 6.4: Maximum yield as a function of the number of electrons for each gas. For all the gases but $K r$, the values are saturation values. For Kr, SEY of 60 monolayers is shown.

Finally, the electron density of different gases is compared. As in figure 6.5 the SEY increases with increasing electron density. Again $\mathrm{CO}$ is an exception. It has the same electron density like $\mathrm{N}_{2}$ but the SEY is much lower. Figure 6.6 shows that the rule for electron density is also valid for rare gases. With increasing electron density, the SEY is also increasing.

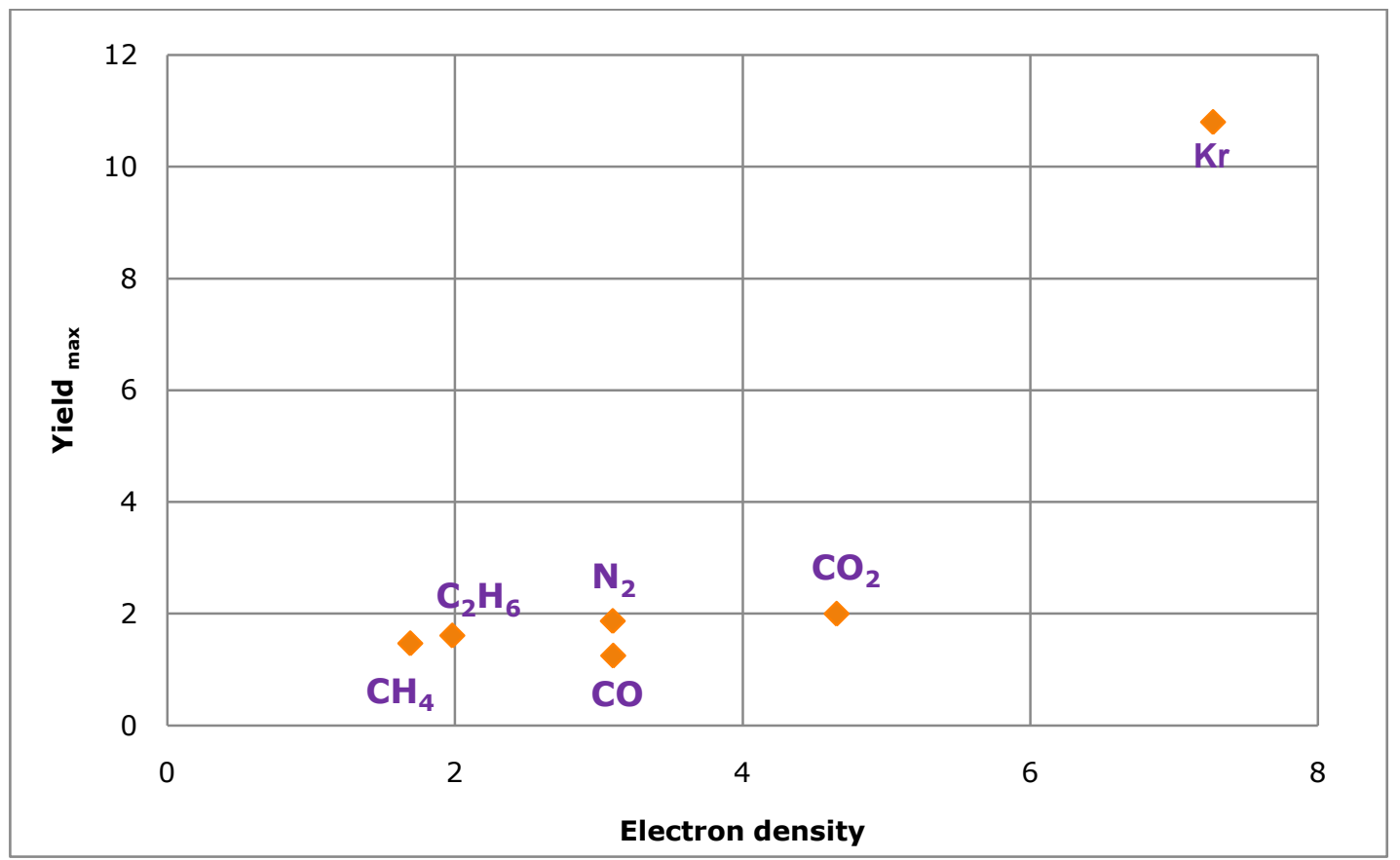

Figure 6.5: Maximum yield as a function of electron density. Value for Kr is taken for $60 \mathrm{ML}$. 


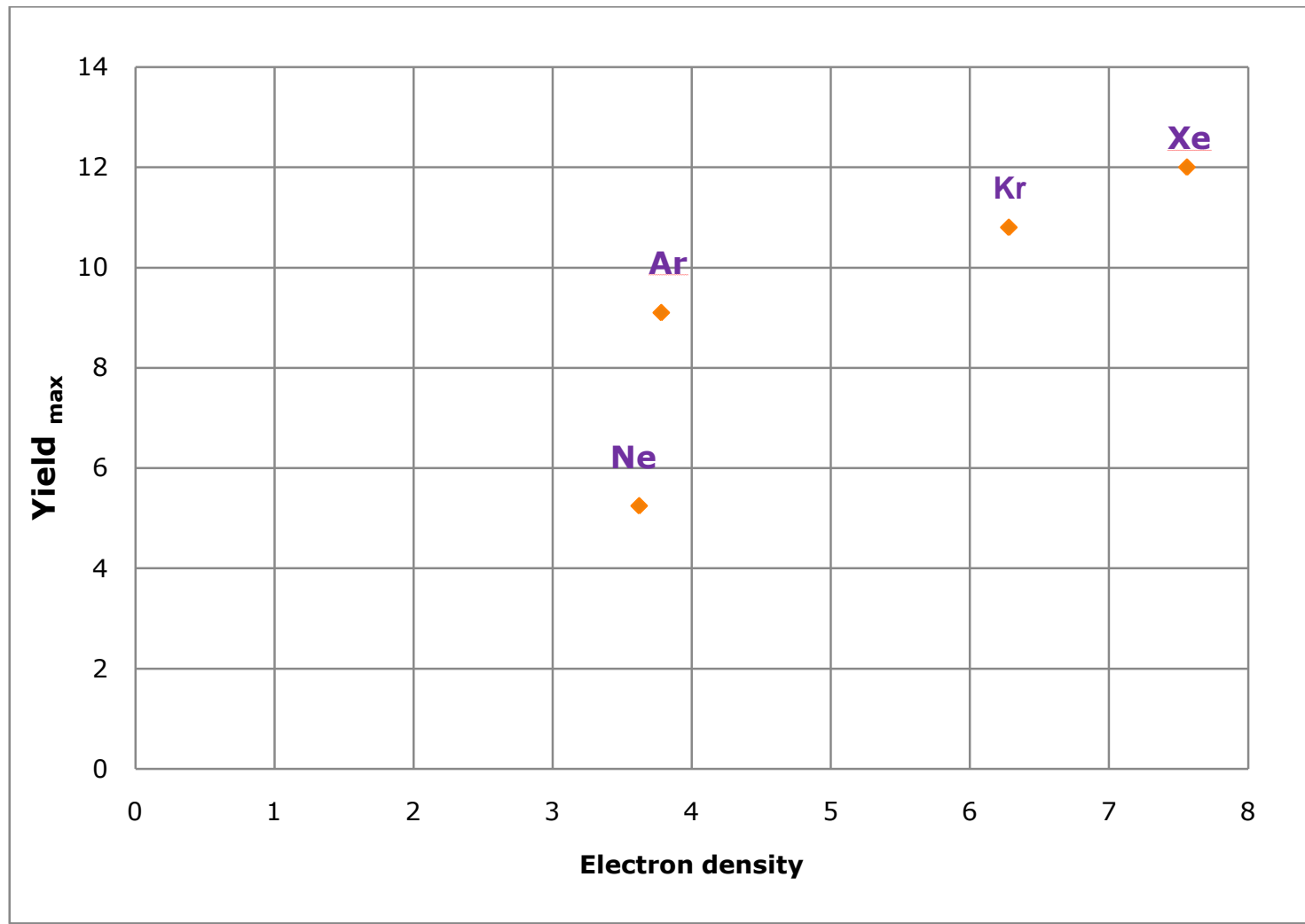

Figure 6.6: Maximum yield of rare gases as a function of electron density. Values are taken for 60 monolayers [30].

\section{$\underline{\text { Range of primary electrons: }}$}

The range of primary electrons is, like the stopping power, a function of density. It can be calculated with the following:

$$
R(n m)=C E_{0}^{n}(k e V)=\frac{115}{\rho} E_{0}^{1.35}
$$

where $\mathrm{n}$ is chosen to be 1.35 when the primary electron energy is less than $5 \mathrm{keV}$. C is a material constant and is equal to $115 / \mathrm{\rho}$ [30]. The density is given in $\mathrm{g} / \mathrm{cm}^{3}$. With increasing density, the range of primary electrons is decreasing, which means that the secondary electrons are generated in an area closer to the surface. Therefore the possibility of the secondary electrons to escape into vacuum is higher, which increases the SEY (Figure 6.7). 


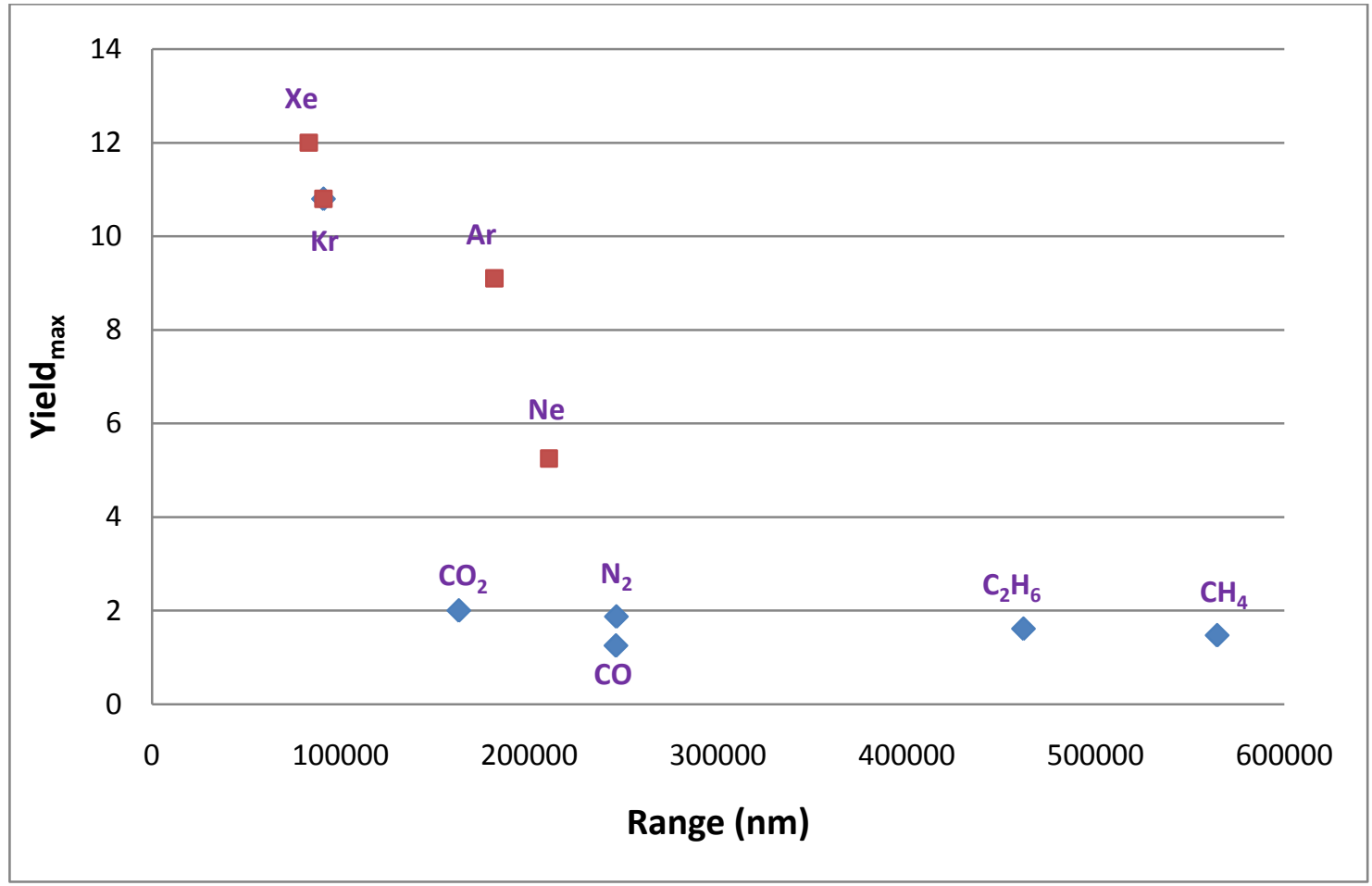

Figure 6.7: Maximum yield of different adsorbates as a function of the range of primary electrons. Values of rare gases are taken for 60 monolayers. For values of $\mathrm{Ne}, \mathrm{Ar}, \mathrm{Xe}$ see [30].

In Figure 6.8 the maximum yield is shown as a function of the density. It is clear that the yield is increasing with increasing density. Carbon monoxide shows again more difference compared to the other gases. It has the same density as $\mathrm{N}_{2}$ but the yield is much lower.

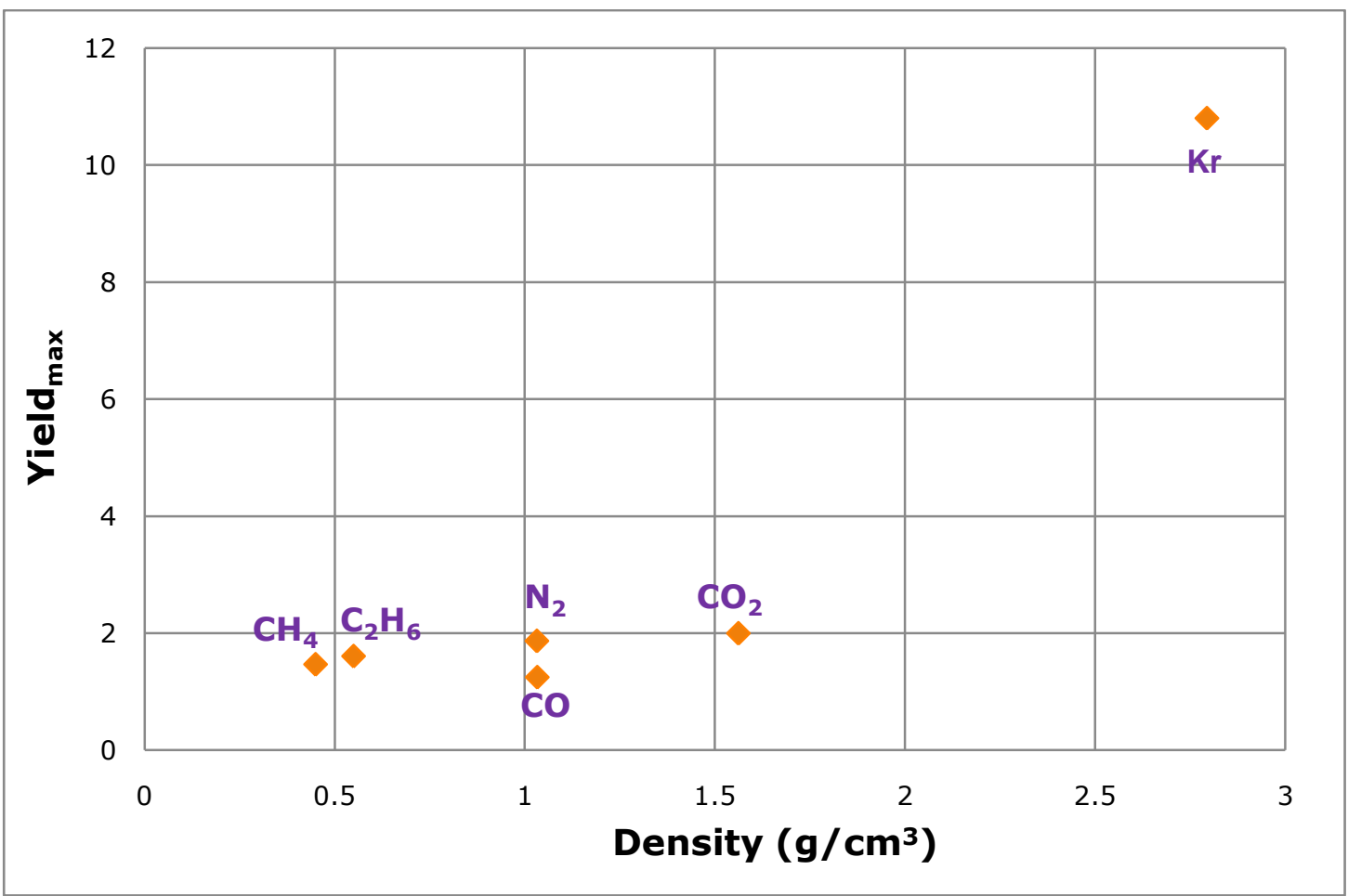

Figure 6.8: Maximum yield as a function of the density. Value for Kr is taken for $60 \mathrm{ML}$. 
The transport of the secondary electrons to the surface is influenced by:

- Inelastic mean free path $\propto$ Valence electrons, band gap, density of solid $\rho$ and molecular weight $M$

\section{$\underline{\text { Inelastic mean free path: }}$}

Not all the generated secondary electrons can leave the surface. Secondary electrons undergo elastic and inelastic collisions during their transport after excitation. The inelastic collisions can reduce the energy of the electrons down to a limit where they can no longer escape the solid. A low inelastic mean free path of secondary electrons will therefore limit the SEY.

In metals, the secondary electrons lose energy by interacting with conduction electrons, lattice vibrations and defects. Due to the large number of conduction electrons, there is a high collision possibility. Since there are no conduction electrons in insulators, the secondary electrons lose energy mainly through excitation of electrons from the valence into the conduction band.

The inelastic mean free path (IMFP) of low energy electrons is influenced by the band gap. The wide gap prevents secondary electrons with kinetic energies less than $E_{\text {gap }}$ from participating in electron-electron collisions [50]. For these electrons, electron-phonon and electron-impurity collisions are mainly responsible for the energy loss. Because of the absence of electron-electron scattering, the secondary electron loses much less energy as it moves through material and the inelastic mean free path becomes large. Therefore in general, wide band gap means long inelastic mean free path of secondary electrons, a fact which has the tendency to increase the SEY (Figure 6.9). In table 6.1 it is clear that adsorbates with high yields have high band gaps but still there is not a perfect correlation. Krypton, which has the highest yield of them, has a lower band gap than $N_{2}$, and $N_{2}$, which has a lower yield than $\mathrm{CO}_{2}$ has a higher band gap. $\mathrm{CH}_{4}$ and $\mathrm{CO}$ have much lower band gaps compared with $\mathrm{Kr}, \mathrm{N}_{2}$ and $\mathrm{CO}_{2}$ and they have very low yields.

It is known, that rare gases have very high yields. They have also very wide band gaps. But $\mathrm{Ne}$, the one with the lowest yield has the widest band gap of them [51] and Xe has the highest yield and the lowest band gap. To have a wide band gap increases the SEY but it is not the only factor changing the SEY.

The IMFP is also influenced by the number of valence electrons (Figure 6.10), density (figure 6.6) and molecular weight, according to the parameters used in the model of reference Tanuma Penn Powell [22]. 
In Figure 6.9 the inelastic mean free path of electrons, calculated with this model, in different adsorbates is shown as a function of the energy. It is again possible to see that inelastic mean free path of electrons cannot be the only factor changing the SEY. Again $\mathrm{N}_{2}, \mathrm{CO}_{2}$ and $\mathrm{Kr}$ have bigger IMFP than $\mathrm{CO}$ and $\mathrm{CH}_{4}$ but $\mathrm{Kr}$ with the highest SEY has smaller IMFP than $\mathrm{N}_{2}$ and $\mathrm{CO}_{2}$. Also $\mathrm{N}_{2}$ has a lower yield than $\mathrm{CO}_{2}$ but it has a bigger IMFP than $\mathrm{CO}_{2}$. Note that in general the model is valid only above certain energies, typically $30 \mathrm{eV}$ and therefore it does not completely cover the range of secondary electrons. In contrast to the curve, the IMFP is expected to increase for the low energies compared to the curve.

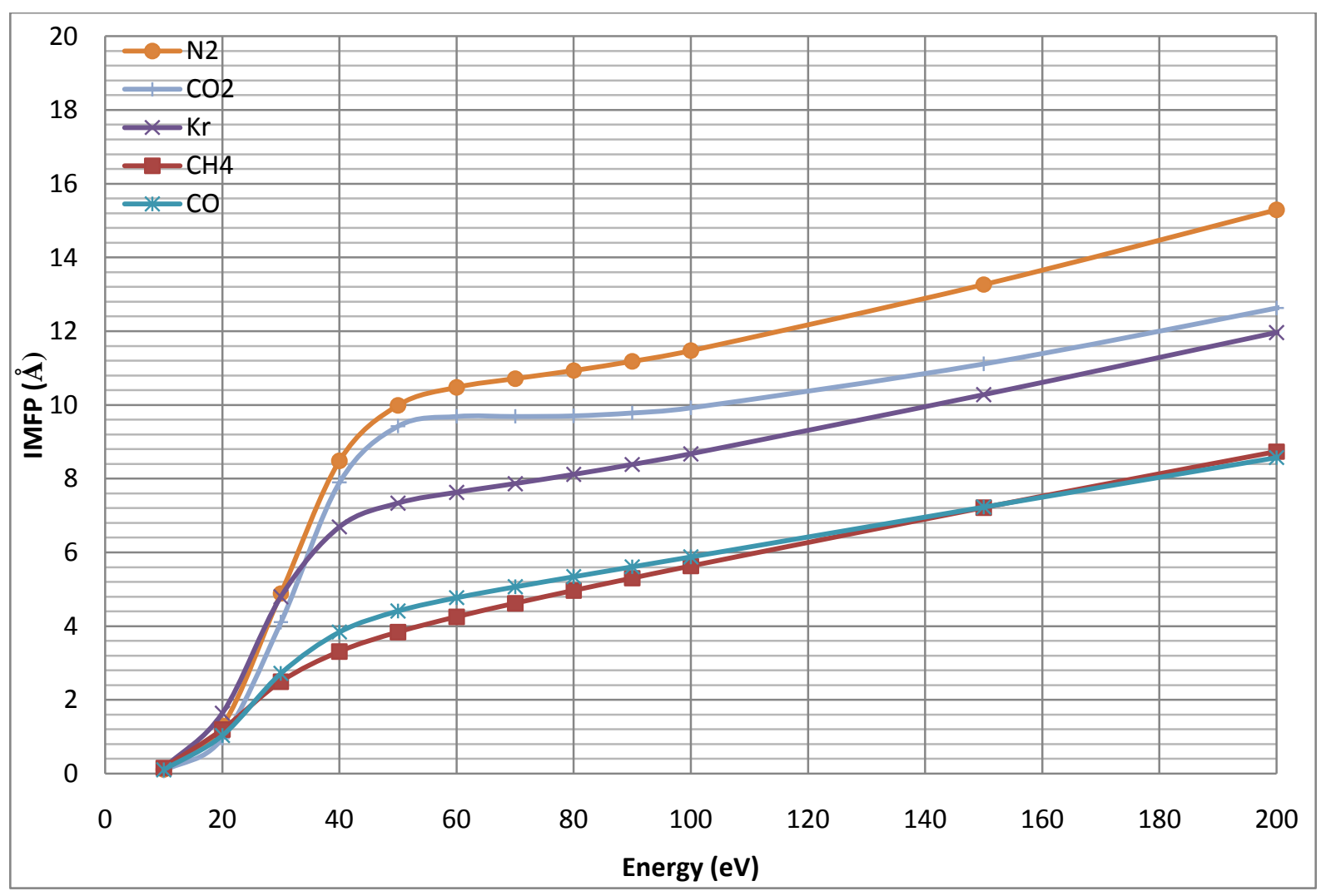

Figure 6.9: Inelastic mean free path of different adsorbates as a function of the energy. The values are calculated using [22]. The value for $\mathrm{Kr}$ is for $60 \mathrm{ML}$.

Figure 6.10 shows the maximum yield as a function of the number of valence electrons. Here it is clear that the curve doesn't follow any rule. 


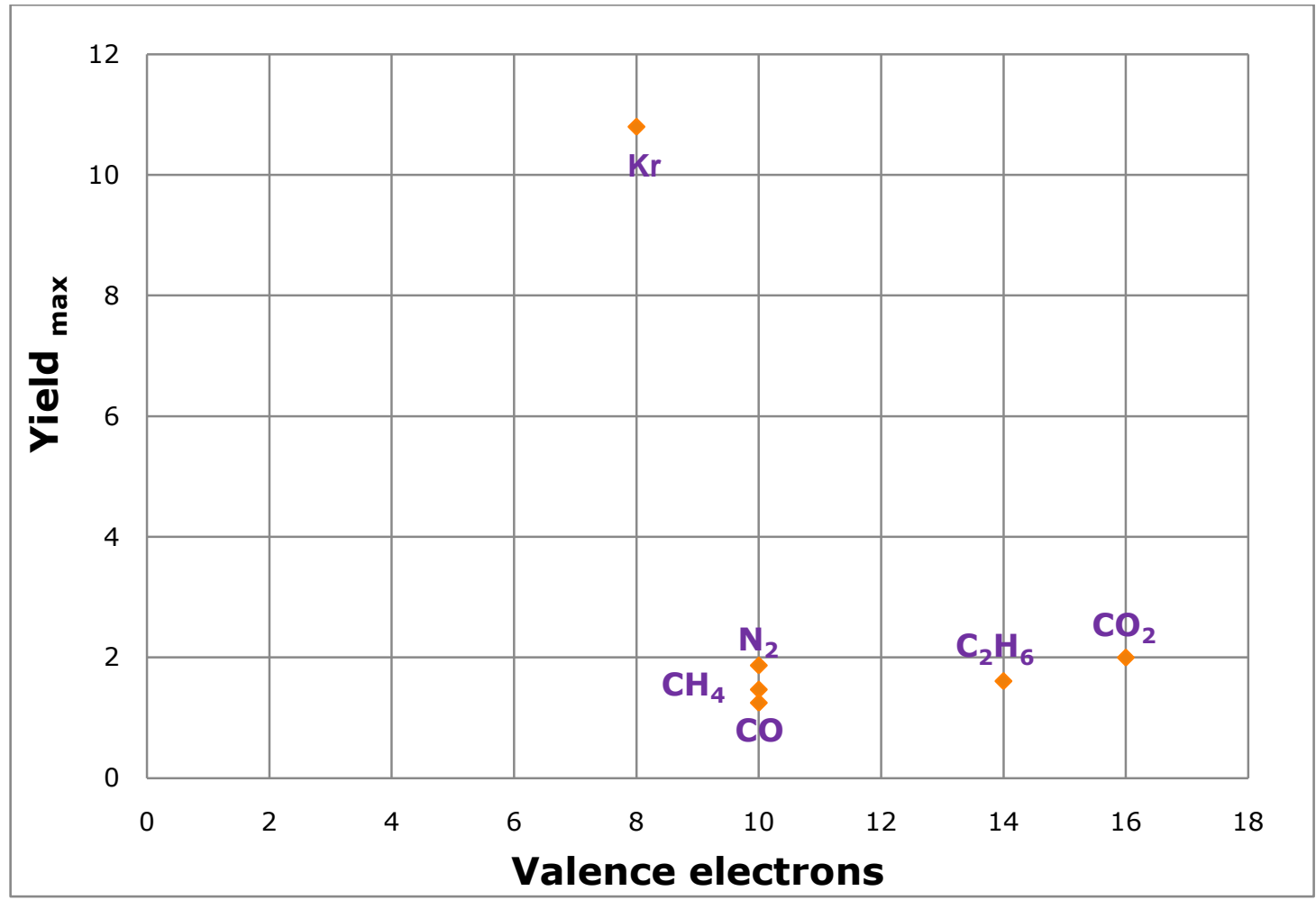

Figure 6.10: Maximum yield as a function of the number of valence electrons. The value for $\mathrm{Kr}$ is for $60 \mathrm{ML}$.

To summarize all; SEY is a phenomena which is affected by many different factors. In order to understand the behaviour of different materials, many properties of that material have to be investigated. An interesting aspect is that some parameters can have self-contradictory effects as in case of band gap and density.

Band gap is affecting the total number of secondary electrons in two different ways. On one hand, wide band gaps mean high average energy for creation of electron hole pairs. Therefore it is decreasing the number of generated secondary electrons. On the other hand, wide band gap prevents secondary electrons from undergoing inelastic collisions with valence electrons and accordingly it helps to the secondary electrons for keeping their energy.

Density is also a parameter which affects the SEY in two different ways. On one hand, high density causes a low range for the primary electrons, so the total energy is dissipated in a small area close to surface. That way it helps to increase the SEY. On the other hand with increasing density, inelastic mean free path of the secondary electrons is decreasing and therefore SEY is decreasing as well. 


\subsection{Conditioning}

The bombardment of an electro polished substrate with 25 monolayers of physisorbed water decreased the yield due to desorption of the adsorbed water. After desorbing all the water layers from the surface, the yield continues decreasing. With an electron dose of $1.5 \cdot 10^{-2}$ $\mathrm{C} / \mathrm{mm}^{2}$ the yield reaches its minimum of 1.36 . This yield is even smaller than the yield of the electro-polished copper before water adsorption. This is a sign for the formation of the carbonaceous layers on the surface [32]. The carbon generated on the surface due to the electron bombardment comes from the substrate itself.

The bombardment of electro-polished copper with 25 monolayers of carbon monoxide increases the yield slightly for a very short moment due to the desorption of the carbon monoxide, which has a lower yield than the copper. But it decreases again with continuing conditioning. It reaches an even lower yield than the conditioning with water. The amount of the generated carbon comes from the substrate, due to the dissociation of the hydrocarbons on the surface and from the adsorbate, due to the dissociation of the carbon monoxide adsorbed on the surface. Therefore we get more carbon layers on the surface than in case of the conditioning with water. In this way it is possible to reach much lower yields (1.21), in this case.

The last measurements with conditioning were performed with 30 monolayers of $N_{2}$. Although that time, the measurements have been done with an already pre- bombarded substrate. The same substrate used during the conditioning with carbon monoxide was left in vacuum for 26 hours before being used for the measurements with $N_{2}$. The yield of the copper substrate at the beginning of the measurements was 1.45. With adsorption of $\mathrm{N}_{2}$ it increases to 2.01 . Already with a dose of $1.53 \cdot 10^{-3} \mathrm{C} / \mathrm{mm}^{2}$, the yield decreases rapidly to 1.56. With increasing dose, it continues decreasing. However this time it doesn't reach so low values as in the case of conditioning with carbon monoxide. The final yield is about the same value as in the case of water (1.35). This could be the confirmation about the dissociation of physisorbed carbon monoxide during electron bombardment.

Overall it has been shown that the SEY varies with the bombarding electron dose due to the cleaning of the surface and to the formation of carbonaceous layers on the surface. 


\section{Conclusion}

This work was carried out in the vacuum group of the TE department at CERN. Its aim was to investigate the secondary electron emission of cryogenic surfaces as a function of physisorbed gases.

This work has presented the secondary electron yield of physisorbed $\mathrm{N}_{2}, \mathrm{CO}, \mathrm{CO}_{2}, \mathrm{CH}_{4}$, $\mathrm{C}_{2} \mathrm{H}_{6}$ and $\mathrm{Kr}$ at liquid helium temperature on a copper, aluminium and electro-polished copper. Besides the yield of physisorbed gases on different substrates, measurements about the secondary electron yield of niobium and carbon have been carried out. Thanks to the experimental setup and procedure used, allowing a simple definition of pumping speed and coverage, the presented results could be obtained. It has been seen that the behaviour of the SEY could be divided in 3 parts. The first part was related to the thin layers, where the adsorbate layers was not effective enough in producing secondary electrons but it was effective to attenuate the secondary electrons generated in the substrate. The second part was about the intermediate thickness. Here the total SEY was the sum of the secondaries generated in the adsorbate and the secondaries generated in the substrate. In the intermediate case the adsorbate was getting more and more important whereas the substrate was losing its influence. The last part was about the thick layers, where the substrate was not effective anymore and the adsorbate was the dominant factor in the total SEY. The measurements with Niobium has shown that the superconductivity doenst have any influence in the SEY. Is has been shown that carbon could be a potential solution to eliminate the e-cloud in accelerators due to its low SEY. Due to the cleaning of the surface and formation of carbonaceous layers on the substrate, conditioning is also a solution to decrease the e-cloud effect.

The goal of this work was to outline the common points of the physisorbed gases in order to give a general explanation of the secondary electron yield. 


\section{Bibliography}

1. http://www2.slac.stanford.edu/vvc/theory/model.html. The Standard Model.

2. http://public.web.cern.ch/public/en/science/StandardModel-en.html, The Standard Model.

3. D.A.Greenwood, W.N.C.a., An Introduction to the Standard Model of Particle Physics. 1998, Cambridge: Cambridge Universith Press.

4. http://www.cern.ch. CERN-European Organization for Nuclear Research. 2010.

5. http://public.web.cern.ch/public/en/LHC/LHC-en.html. The Large Hadron Collider.

6. T.S.Pettersson, a.P.L., The Large Hadron Collider, Conceptual Design. 1995, CERN/AC/95-05: Geneva.

7. L.R.Evans, The large hadron collider project. CERN, 1996.

8. E.Keil, The CERN Large Hadron Collider LHC, CERN: Geneva.

9. Colloboration, A., ATLAS: technical proposal for a general-pirpose pp experiment at the Large Hadron Collider at CERN. 1994. Proposal CERN-LHCC-94-43.

10. Coughlan, G.D. and J.E. Dodd, The ideas of particle physics- An introduction for scientists. 1991.

11. Colloboration, C., CMS, the Compact Muon Solenoid: technical proposal. 1994, CERN: Geneva.

12. colloboration, A., ALICE: Technical proposal for a Large Ion collider Experiment at the CERN LHC. 1995, CERN: Geneva.

13. colloboration, L., LHCb: Technical Proposal. 1998, CERN: Geneva.

14. M.A.Furman, The electron-cloud effect in the arcs of the LHC, in LHC Project Report. 1998, CERN: Geneva.

15. J.Wenninger. Luminosity Diagnostics. 2008.

16. Benvenuti, C., A. Escudeiro Santana, and V. Ruzinov, Ultimate pressures achieved in TiZrV sputter-coated vacuum chambers. 2001. 60(2).

17. Wilson, E.J.N., An Introduction to Particle Accelerators. 2001.

18. Gröbner, O., The LHC vacuum system. 1998, CERN: Geneva.

19. H.Bruining, Physics and Applications of secondary Electron emission. 1954, Londen: Pergamon Press.

20. H.Seiler, Secondary electron emission in the scanning electron microscope. Journal of Applied Physics, 1983. 54: p. R1-R18.

21. H.Seiler, Z.Angew.Phys., 1967. 22(249).

22. S.Tanuma, C.J.Powell, and D.R.Penn, Material dependence of electron inelastic mean free paths at low energies. J. Vac. Sci. Technol. A, 1990. 8(3): p. 2213-2216.

23. D.C.Joy, A model for calculating secondary and backscattering electron yields. Journal of Microscopy, 1987. 147: p. 51-64.

24. K.Kanaya, S.Ono, and F.Ishigaki, Secondary electron emission from insulators. Journal of Physics D, 1978. 11(2425): p. 2425-2437.

25. J.R.Young, Penetration of electrons in $\mathrm{Al}_{2} \mathrm{O}_{3}$-films Physical Review, 1956. 103: p. 292-293.

26. G.Kuhnle, H.S.a., Z.Angew.Phys., 1970. 29(254).

27. K.Nishimura, T.Itotani, and K.Ohya, Influence of surface roughness on secondary electron emission and electron backscattering from metal surface. Japanese Journal of Applied Physics, 1994. 33: p. 4727-4734.

28. C.Scheuerlein, The Influence of an Air Exposure on the Secondary Electron Yield of Copper, in CERN-2002-022. 1997, University of Surrey: Geneva. 
29. I.Buchholz, Zur Lage des Maximums der Ausbeutekurve bei der Sekundärelektronenemission. Z.Physik, 1969.

30. J.Cazaux, Y.Bozhko, and N.Hilleret, Electron-induced secondary electron emission yield from condensed rare gases: $\mathrm{Ne}, \mathrm{Ar}, \mathrm{Kr}$ and Xe. Physical Review B, 2005. 71(035419).

31. H.J.Hopman and J.Verhoeven, Secondary electron emission data of cesiated oxygen free high conductivity copper. Applied Surface Science, 1999. 150: p. 1-7.

32. B.Henrist, et al., The variation of the secondary electron yield and of the desorption yield of copper under electron bombardment: origin and impact on the conditioning of the LHC, in LHC Project Report. 2002, CERN: Geneva.

33. O.Brüning, F.C., I.R.Collins, O.Gröbner, B.Henrist, N.Hilleret, J.-M.LAurent, M.Morvillo, M.Pivi, F.Ruggiero, X.Zhang. Electron Cloud and Beam Scrubbing in the LHC. in Particle Accelerator Conference. 1999. New York.

34. O.Bruning, et al., LHC Design Report Vol.1: The LHC Main Ring, in CERN-2004-003. 2004, CERN: Geneva.

35. F.Zimmermann, Electron-Cloud Effects in the LHC, in ECLOUD'02 workshop, G.Rumolo and F.Zimmermann, Editors. 2002, CERN: CERN, Geneva, Switzerland.

36. M.Sands, The Physics of Electron Storage Rings: An Introduction, in SLAC-121. 1970, Stanford University.

37. F.Zimmermann, A Simulation Study of Electron-Cloud Instability and Beam-Induced Multipacting in the LHC, in CERN LHC Project Report. 1997.

38. J.Kouptsidis and G.A.Mathewson, in Internal Report. 1976, DESY.

39. http://www.crxo.lbl.gov/optical constants/, LBNL X-Ray laboratory.

40. G.Rumolo and F.Zimmermann, Electron cloud simulations: beam instabilities and wakefields. Physical Review Special Topics - Accelerators and beams, 2002. 5(121002).

41. K.Ohmi and F.Zimmermann, Head- Tail instability caused by electron clouds in positron storage rings. Physical Review Letters, 2001. 85(3821).

42. G.Rumolo, F.Ruggiero, and F.Zimmermann, Phys. Rev. ST Accel. Beams 2002. 4(012801).

43. W.Umrath, ed. Fundamentals of Vaccum Technology. 2001/2002, Leybold Vacuum.

44. N.Marquardt. Introduction to the principles of vacuum physics. in CAS Cern accelerator school- Vacuum Technology. 1999. Scanticon Conference Centre, Snekersten, Denmark: CERN.

45. A.Berman, Vacuum engineering calculations, formulas and solved exercises. 1992: Academis Press, Inc.

46. R.F.Barron, Cryogenic Systems. 1985, New york: Oxford University Press, Inc.

47. H.Tratnik, Electron Stimulated Desorption of Condensed Gases on Cryogenic Surfaces, in Institut für Allgemeine Physik. 2005, Technische Universität Wien: Vienna.

48. R.C.Alig and S.Bloom, Electron-Hole-Pair Creation Energies in Semiconductors. Physical Review Letters, 1975. 35(22): p. 1522-1525.

49. http://physics.nist.gov/PhysRefData/Star/Text/ESTAR.html. Stopping power and range tables for electrons.

50. A.Shih, et al., Secondary electron emission studies. Applied Surface Science, 1997. 111: p. 251-258.

51. M.L.Klein and J.A.Venables, Rare gas solids. Vol. 2. 1977: Academic Press. 


\section{Acknowledgements}

Three years of hard work are presented in this thesis, which would not have been possible without the help of many people. Therefore, some words of gratitude are in order.

First of all, I would like to thank my supervisors, Mauro Taborelli at CERN and Herbert Störi at the Vienna University of Technology for their academic guidance.

I would also like to thank Holger Neupert for all his technical support and guidance. Further I would like to thank Giovanna Vandoni and Bernard Henrist for their guidance during the early stage of my thesis.

Finally I would like to thank my mother Tuba, my father Alpoğuz and my sister Aslıhan for their never-ending belief in me. 


\section{Curriculum Vitae}

Personal information

Asena Kuzucan
Date of birth: 13 June 1983

Nationality: Turkish

E-mail: asena_kuzucan@hotmail.com

Telephone :+(41) 793876672

CERN TE-VSC-SCC

Vienna University of Technology

Doctor of Technical Science (PhD)

$2002-2007$

- Institution:

Vienna University of Technology

- Field of study:

Technical Physics

- Specialization:

Applied Physics

- Acquired title:

Master of Science (Dipl.-Ing.)

$1994-2002$

- Name of the high school:

Austrian Sankt Georg's-School Istanbul

- Focus in studies:

Science

\section{Research Experience}

Date

Concept

2011

PhD Thesis Project (at CERN)

Thesis title: Secondary electron emission yield on cryogenic surfaces as a function of physisorbed gases

Final Year Thesis Project

Thesis Title: Investigations of the total sputter yield of fusion-relevant materials under ion bombardment utilizing a novel Quartz-Crystal-Microbalance

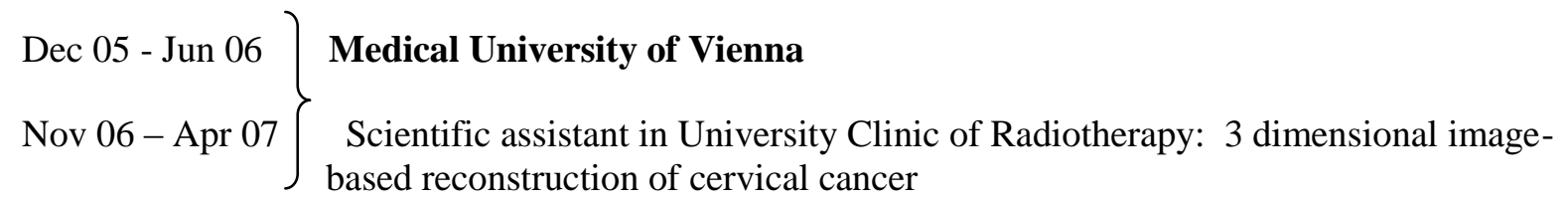




\section{Papers and conferences}

2011

Student grant for IPAC11 - Poster Presentation

2011 Proceedings to IPAC11: "Secondary electron yield on cryogenic surfaces as a function of physisorbed gases"

"Characterization of amorphous carbon coatings with low secondary electron yield"

2011

Speaker at "IAP Seminar" Vienna University of Technology / Austria

2010

Poster: Doctoral Student Poster Session \CERN- Switzerland

2009

Paper: "Ion-induced erosion of tungsten surfaces studied by a sensitive quartzcrystal- microbalance technique"

Journal of Nuclear Materials 390-391 (2009) 1102-1105

Contribution to: "Consequences of random and systematic reconstruction uncertainties in 3D Image-based brachytherapy in cervical cancer " Radiotherapy and Oncology Volume 89 Issue 2

\section{Languages}

Turkish: Mother Tongue

German: Excellent (written and spoken)

English: Excellent (written and spoken)

Spanish: Intermediate (written and spoken)

French: Intermediate (written and spoken)

\section{Computer knowledge}

Operating systems: Windows 7, Windows XP, Windows Vista, Mac OSX, Linux

Programming: FORTRAN, C

Others: LabVIEW, MS Excel, MS PowerPoint, MS Word, MS Visio, AutoCAD, KaleidaGraph 